\title{
Enantioselective Bromo-oxycyclization of Silanol
}

Zilei Xia, Jiadong Hu, Zhigao Shen, Xiaolong Wan, Qizheng Yao, Yisheng Lai, Jin-Ming Gao and Weiqing Xie,*

\section{Table of contents}

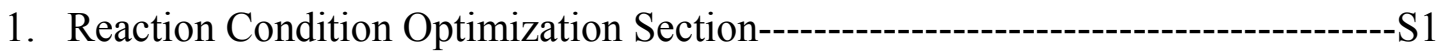

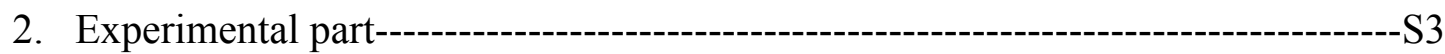

3. References-----------------------------------------------------------------------------S28

4. Plausible catalytic cycle and transition sate-----------------------------------------S29

5. Copies of NMR spectra of new compounds------

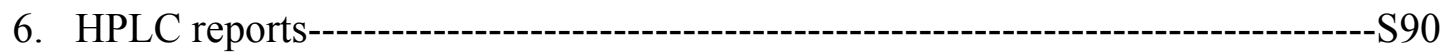

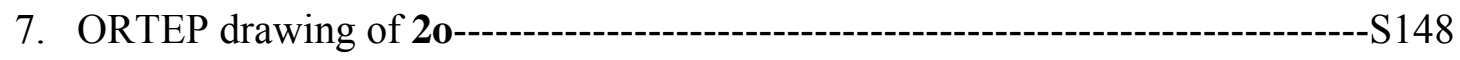




\section{Reaction condition optimization section ${ }^{[1,2]}$}<smiles>C=C(C)c1ccccc1[Si](O)(c1ccccc1)c1ccccc1</smiles>

1a<smiles>C[C@]1(CBr)O[Si](c2ccccc2)(c2ccccc2)c2ccccc21</smiles>

$2 a$<smiles>[R7]c1cc2c(c(-c3c([R])cc4c(c3OP(=O)(O)O)CCCC4)c1OP(=O)(O)O)CCCC2</smiles>

L1 R"=2,4,6-trisisopropylphenyl<smiles></smiles>

L2: $\mathbf{R}^{\prime}=\mathrm{H}$

L3: $\mathrm{R}^{\prime}=n-\mathrm{C}_{8} \mathrm{H}_{17}$ L4: $\mathrm{R}^{\prime}=n-\mathrm{C}_{18} \mathrm{H}_{37}$ L5: R'=TIPS

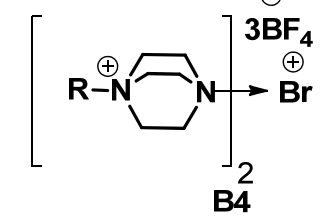

$\mathrm{R}=3,5$-bistrifluoromethylbenzyl<smiles>O=P(O)(Oc1cc2ccccc2cc1Br)Oc1c(Br)cc2ccccc2c1Br</smiles>

L6: Ar=9-anthryl L7: Ar=3,5-ditrifluoromethylphenyl L8: Ar=9-phenanthryl

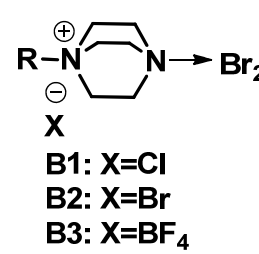

Table S1. Screening of Bromine sources and Catalysts

\begin{tabular}{ccccccccc}
\hline entry & solvent & $\begin{array}{c}\text { catalyst } \\
\text { (equiv.) }\end{array}$ & $\begin{array}{c}\text { base } \\
\text { (equiv.) }\end{array}$ & $\begin{array}{c}\mathrm{Br}^{+} \\
(\text {equiv. })\end{array}$ & $\begin{array}{c}\mathrm{T} \\
\left({ }^{\circ} \mathrm{C}\right)\end{array}$ & $\begin{array}{c}\text { time } \\
(\mathrm{h})\end{array}$ & $\begin{array}{c}\text { yield } \\
(\%)\end{array}$ & $\begin{array}{c}\text { ee } \\
(\%)\end{array}$ \\
\hline 1 & Toluene & $\mathbf{L 1}(0.10)$ & $\mathrm{NaHCO}_{3}(4.0)$ & $\mathbf{B} 1(1.3)$ & 0 & 6 & 81 & 92 \\
2 & Toluene & $\mathbf{L 1}(0.10)$ & $\mathrm{NaHCO}_{3}(4.0)$ & $\mathbf{B} 2(1.3)$ & 0 & 12 & 64 & 81 \\
3 & Toluene & $\mathbf{L 1}(0.10)$ & $\mathrm{NaHCO}_{3}(4.0)$ & $\mathbf{B 3}(1.3)$ & 0 & 12 & 40 & 75 \\
4 & Toluene & $\mathbf{L 1}(0.10)$ & $\mathrm{NaHCO}_{3}(4.0)$ & $\mathbf{B} 4(1.3)$ & 0 & 12 & 0 & $\mathrm{NR}$ \\
5 & Toluene & $\mathbf{L 2}(0.10)$ & $\mathrm{NaHCO}_{3}(4.0)$ & $\mathbf{B 1}(1.3)$ & 0 & 6 & 70 & 88 \\
6 & Toluene & $\mathbf{L 3}(0.10)$ & $\mathrm{NaHCO}_{3}(4.0)$ & $\mathbf{B 1}(1.3)$ & 0 & 6 & 77 & 83 \\
7 & Toluene & $\mathbf{L 4}(0.10)$ & $\mathrm{NaHCO}_{3}(4.0)$ & $\mathbf{B 1}(1.3)$ & 0 & 6 & 89 & 85 \\
8 & Toluene & $\mathbf{L 5}(0.10)$ & $\mathrm{NaHCO}_{3}(4.0)$ & $\mathbf{B 1}(1.3)$ & 0 & 6 & 81 & 90 \\
9 & Toluene & $\mathbf{L 6}(0.10)$ & $\mathrm{NaHCO}_{3}(4.0)$ & $\mathbf{B 1}(1.3)$ & 0 & 10 & 84 & 25 \\
10 & Toluene & $\mathbf{L 7}(0.10)$ & $\mathrm{NaHCO}_{3}(4.0)$ & $\mathbf{B 1}(1.3)$ & 0 & 10 & 79 & 13 \\
11 & Toluene & $\mathbf{L 8}(0.10)$ & $\mathrm{NaHCO}_{3}(4.0)$ & $\mathbf{B 1}(1.3)$ & 0 & 10 & 80 & 16 \\
\hline
\end{tabular}

[a] Isolated yield. [b] Determined by HPLC on OD-H column. 
Table S2. Screening of Solvents

\begin{tabular}{ccccccccc}
\hline entry & solvent & $\begin{array}{c}\text { catalyst } \\
\text { (equiv.) }\end{array}$ & $\begin{array}{c}\text { base } \\
\text { (equiv.) }\end{array}$ & $\begin{array}{c}\mathrm{Br}^{+} \\
\text {(equiv.) }\end{array}$ & $\begin{array}{c}\mathrm{T} \\
\left({ }^{\mathrm{o}} \mathrm{C}\right)\end{array}$ & $\begin{array}{c}\text { time } \\
(\mathrm{h})\end{array}$ & $\begin{array}{c}\text { yield } \\
(\%)\end{array}$ & $\begin{array}{c}\text { ee } \\
(\%)\end{array}$ \\
\hline 1 & Hexane & $\mathbf{L 1}(0.10)$ & $\mathrm{NaHCO}_{3}(4.0)$ & $\mathbf{B 1}(1.3)$ & 0 & 20 & 53 & 93 \\
2 & Xylene & $\mathbf{L 1}(0.10)$ & $\mathrm{NaHCO}_{3}(4.0)$ & $\mathbf{B 1}(1.3)$ & 0 & 3 & 71 & 96 \\
3 & Benzene & $\mathbf{L 1}(0.10)$ & $\mathrm{NaHCO}_{3}(4.0)$ & $\mathbf{B 1}(1.3)$ & 20 & 4 & 61 & 91 \\
4 & $\mathrm{Et}{ }_{2} \mathrm{O}$ & $\mathbf{L 1}(0.10)$ & $\mathrm{NaHCO}_{3}(4.0)$ & $\mathbf{B 1}(1.3)$ & 0 & 5 & 66 & 91 \\
5 & EtOAc & $\mathbf{L 1}(0.10)$ & $\mathrm{NaHCO}_{3}(4.0)$ & $\mathbf{B 1}(1.3)$ & 0 & 4 & 65 & 70 \\
6 & $\mathrm{DCM}$ & $\mathbf{L 1}(0.10)$ & $\mathrm{NaHCO}_{3}(4.0)$ & $\mathbf{B 1}(1.3)$ & 0 & 3 & 0 & complex \\
7 & $\mathrm{CCl}$ & $\mathbf{L 1}(0.10)$ & $\mathrm{NaHCO}_{3}(4.0)$ & $\mathbf{B 1}(1.3)$ & 0 & 5 & 71 & 91 \\
\hline
\end{tabular}

[a] Isolated yield. [b] Determined by HPLC on OD-H column.

Table S3. Screening of Bases

\begin{tabular}{ccccccccc}
\hline entry & solvent & $\begin{array}{c}\text { catalyst } \\
\text { (equiv.) }\end{array}$ & $\begin{array}{c}\text { base } \\
\text { (equiv.) }\end{array}$ & $\begin{array}{c}\mathrm{Br}^{+} \\
\text {(equiv.) }\end{array}$ & $\begin{array}{c}\mathrm{T} \\
\left({ }^{\circ} \mathrm{C}\right)\end{array}$ & $\begin{array}{c}\text { time } \\
(\mathrm{h})\end{array}$ & $\begin{array}{c}\text { yield } \\
(\%)^{[\mathrm{a}]}\end{array}$ & $\begin{array}{c}\text { ee } \\
(\%)^{[\mathrm{b}]}\end{array}$ \\
\hline 1 & Toluene & $\mathbf{L 1}(0.10)$ & $\mathrm{Na}_{2} \mathrm{CO}_{3}(4.0)$ & $\mathbf{B 1}(1.3)$ & 0 & 4 & 81 & 96 \\
2 & Toluene & $\mathbf{L 1}(0.10)$ & $\mathrm{K}_{2} \mathrm{CO}_{3}(4.0)$ & $\mathbf{B 1}(1.3)$ & 0 & 4 & 87 & 97 \\
3 & Toluene & $\mathbf{L 1}(0.10)$ & $\mathrm{K}_{3} \mathrm{PO}_{4}(4.0)$ & $\mathbf{B 1}(1.3)$ & 0 & 4 & 77 & 94 \\
4 & Toluene & $\mathbf{L 1}(0.10)$ & $\mathrm{NaOH}_{(4.0)}$ & $\mathbf{B 1}(1.3)$ & 0 & 4 & 0 & complex \\
5 & Toluene & $\mathbf{L 1}(0.10)$ & $\mathrm{Cs}_{2} \mathrm{CO}_{3}(4.0)$ & $\mathbf{B 1}(1.3)$ & 0 & 16 & 49 & 84 \\
\hline
\end{tabular}

[a] Isolated yield. [b] Determined by HPLC on OD-H column.

Table S4. Effect of Temperature

\begin{tabular}{ccccccccc}
\hline entry & solvent & $\begin{array}{c}\text { catalyst } \\
\text { (equiv.) }\end{array}$ & $\begin{array}{c}\text { base } \\
\text { (equiv.) }\end{array}$ & $\begin{array}{c}\mathrm{Br}^{+} \\
\text {(equiv.) }\end{array}$ & $\begin{array}{c}\mathrm{T} \\
\left({ }^{\circ} \mathrm{C}\right)\end{array}$ & $\begin{array}{c}\text { time } \\
(\mathrm{h})\end{array}$ & $\begin{array}{c}\text { yield } \\
(\%)\end{array}$ & $\begin{array}{c}\text { ee } \\
(\%)\end{array}$ \\
\hline 1 & Toluene & $\mathbf{L 1}(0.10)$ & $\mathrm{K}_{2} \mathrm{CO}_{3}(4.0)$ & $\mathbf{B 1}(1.3)$ & 20 & 1.5 & 70 & 95 \\
2 & Toluene & $\mathbf{L 1}(0.10)$ & $\mathrm{K}_{2} \mathrm{CO}_{3}(4.0)$ & $\mathbf{B 1}(1.3)$ & -20 & 4 & 80 & 94 \\
\hline
\end{tabular}

[a] Isolated yield. [b] Determined by HPLC on OD-H column.

Table S5. Influence of Catalyst Loading

\begin{tabular}{ccccccccc}
\hline entry & solvent & $\begin{array}{c}\text { catalyst } \\
\text { (equiv.) }\end{array}$ & $\begin{array}{c}\text { base } \\
\text { (equiv.) }\end{array}$ & $\begin{array}{c}\mathrm{Br}^{+} \\
\text {(equiv.) }\end{array}$ & $\begin{array}{c}\mathrm{T} \\
\left({ }^{\circ} \mathrm{C}\right)\end{array}$ & $\begin{array}{c}\text { time } \\
(\mathrm{h})\end{array}$ & $\begin{array}{c}\text { yield } \\
(\%)^{[\mathrm{a}]}\end{array}$ & $\begin{array}{c}\text { ee } \\
(\%)^{[\mathrm{b}]}\end{array}$ \\
\hline 1 & Toluene & $\mathbf{L} 1(0.05)$ & $\mathrm{K}_{2} \mathrm{CO}_{3}(4.0)$ & $\mathbf{B 1}(1.3)$ & 0 & 24 & 45 & 96 \\
2 & Toluene & $\mathbf{L} 1(0.15)$ & $\mathrm{K}_{2} \mathrm{CO}_{3}(4.0)$ & $\mathbf{B 1}(1.3)$ & 0 & 1.5 & 73 & 95 \\
\hline
\end{tabular}

[a] Isolated yield. [b] Determined by HPLC on OD-H column. 


\section{Experimental part}

General Information. Unless otherwise specified, all reagents purchased from commercial suppliers were used as received. Non-aqueous reactions were conducted under an inert atmosphere of argon in flame-dried glassware. Anhydrous solvents were treated as followed: tetrahydrofuran, toluene and diethyl ether were distilled from benzophenone ketyl under nitrogen atmosphere, dimethylformamide was distilled over calcium hydride under reduced pressure, dichloromethane was distilled from calcium hydride under nitrogen atmosphere. Thin layer chromatography was conducted on Merck 60 F254 pre-coated silica gel plates. Column chromatography was carried out by normal silica gel (40-60 $\mu \mathrm{m}$, 230-400 mesh, Silicycle P60). NMR data including ${ }^{1} \mathrm{H}$ NMR or ${ }^{13} \mathrm{C}$ NMR spectra were recorded on Mercury 300 and MR 400. ${ }^{1} \mathrm{H}$ NMR Chemical shifts were reported in ppm from the solvent resonance as the internal standard $\left(\mathrm{CDCl}_{3}: 7.27 \mathrm{ppm}\right) .{ }^{13} \mathrm{C}$ NMR chemical shifts were reported in ppm relative to the solvent $\left(\mathrm{CDCl}_{3}: 77 \mathrm{ppm}\right)$. Infrared spectra were performed on a Nicolet 380FT-IR and are reported in terms of frequency of absorption $\left(\mathrm{cm}^{-1}\right)$. Mass spectra were measured on a Shimadzu LCMS-2010EV mass spectrometer (ESI). High resolution mass spectra were obtained from IonSpec 4.7 Tesla FTMS mass spectrometer (MALDI) and Bruker APEXIII 7.0 TESLA FTMS (ESI).

\subsection{Synthesis of substrates 1a-1t}
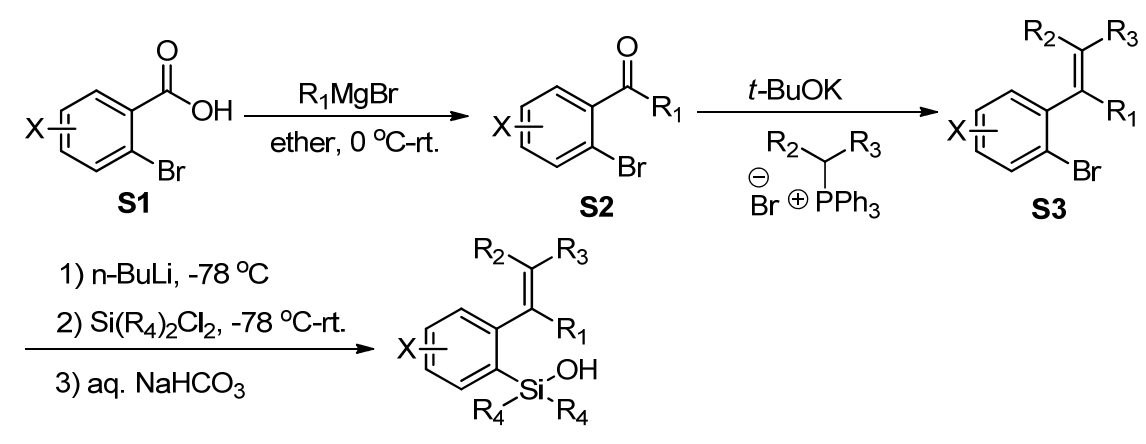

1

\section{General procedure for the synthesis of S2:}

A solution of $\mathrm{R}_{1} \mathrm{MgBr}$ ( $\left.25 \mathrm{mmol}\right)$ in ether was added dropwise to a solution of mixture of $\mathbf{S 1}$ (10 mmol) in anhydrous ether $(50 \mathrm{~mL})$ at $0{ }^{\circ} \mathrm{C}$ under Ar. The mixture was allowed to warm to ambient temperature and stirred overnight. The reaction was quenched by pouring into cooled dilute $\mathrm{HCl}(1 \mathrm{M})$ and the mixture was extracted with ethyl acetate. The organic phases were combined, washed with saturate aq. $\mathrm{Na}_{2} \mathrm{CO}_{3}$ and brine successively, dried over $\mathrm{Na}_{2} \mathrm{SO}_{4}$, and concentrated to afford the crude product. The crude was pure enough for the next step and used for the synthesis of S3 directly without further purification.

\section{General procedure for the synthesis of S3:}

If without specific notes, $\mathbf{S} 3$ were synthesized according to literature with little modificatio. ${ }^{[3,4]}$

To a mixture of Wittig reagent $(15 \mathrm{mmol})$ in $30 \mathrm{~mL}$ THF under Ar was added a solution of $t$-BuOK $(16 \mathrm{mmol})$ in $20 \mathrm{~mL}$ THF at rt., the mixture was stirred for $30 \mathrm{~min}$, then the solution of $\mathbf{S 2}$ in $10 \mathrm{~mL}$ THF was added. The reaction was stirred for additional $2 \mathrm{~h}$ and quenched by pouring into saturate aq. $\mathrm{NH}_{4} \mathrm{Cl}$ and the mixture was extracted with ethyl acetate. The combined organic phases were condensed. The residue was dissolved in $100 \mathrm{~mL} \mathrm{MeOH} / \mathrm{H}_{2} \mathrm{O}(\mathrm{V} / \mathrm{V}=3: 2)$ and the mixture was extracted with petroleum ether. The combined organic phases were washed with $\mathrm{MeOH} / \mathrm{H}_{2} \mathrm{O}(\mathrm{V} / \mathrm{V}=3: 2)(3 \mathrm{X} 200$ 
$\mathrm{mL}$ ) and brine successively, dried over $\mathrm{Na}_{2} \mathrm{SO}_{4}$, and concentrated to afford the crude product. The crude was pure enough for the next step and used for the synthesis of $\mathbf{1}$ directly without further purification.

\section{General procedure for the synthesis of 1:}

The substrates 1 were synthesized according to literature with little modification. ${ }^{[4]}$ A solution of crude S3 got from previous step in $5 \mathrm{~mL}$ of THF was cooled to $-78{ }^{\circ} \mathrm{C}$ under $\mathrm{Ar}$, and $\mathrm{n}$-BuLi $(2.5 \mathrm{M}$ in hexane, $1.2 \mathrm{eq}$ ) was added dropwise. The mixture was stirred for additional $30 \mathrm{mins}$ at same temperature and added to a solution of $\mathrm{Si}\left(\mathrm{R}_{4}\right)_{2} \mathrm{Cl}_{2}\left(2.0\right.$ eq.) in $10 \mathrm{~mL} \mathrm{THF}$ at $-78{ }^{\circ} \mathrm{C}$ under $\mathrm{Ar}$. The mixture was allowed to warm to ambient temperature and stirred overnight. The reaction was quenched by pouring into cooled saturate aq. $\mathrm{NaHCO}_{3}$ and the mixture was extracted with ethyl acetate. The organic phases were combined, washed with brine, dried over $\mathrm{Na}_{2} \mathrm{SO}_{4}$, and concentrated to afford the crude product. The crude product was purified by flash chromatography to afford the target product.<smiles>C=C(C)c1ccccc1[Si](O)(c1ccccc1)c1ccccc1</smiles>

Diphenyl(2-(prop-1-en-2-yl)phenyl)silanol (1a): Following the general procedure, 1a was synthesized from commercial available S2 as slightly yellow oil (2.8 g, 88\% for two steps).

$\mathrm{R} f=0.5$ (petroleum ether/ethyl acetate $=10: 1$ ); IR (film): 3606, 1596, 1428, 1116, 818, 740, 700, 504

$\mathrm{cm}^{-1} ;{ }^{1} \mathrm{H}$ NMR (400 MHz, $\left.\mathrm{CDCl}_{3}\right) \delta$ 7.66-7.57 (m, 4H), 7.47-7.35 (m, 8H), 7.22-7.26 (m, 2H),

5.07-5.09 (m, 1H), 4.87-4.82 (m, 1H), 2.99 (brs, 1H), $1.92(\mathrm{~s}, 3 \mathrm{H}) .{ }^{13} \mathrm{C} \mathrm{NMR}\left(126 \mathrm{MHz}, \mathrm{CDCl}_{3}\right) \delta$

150.83, 149.61, 136.77, 136.42, 135.05, 132.46, 129.85, 129.72, 127.80, 127.56, 126.13, 115.74, 25.34.

HRMS-DART Positive calcd. for $\mathrm{C}_{21} \mathrm{H}_{21} \mathrm{OSi}[\mathrm{M}+\mathrm{H}]^{+}$317.1362, found: 317.1355 .<smiles>C=C(C)c1ccccc1[Si](C)(C)O</smiles>

$1 \mathrm{ab}$

Dimethyl(2-(prop-1-en-2-yl)phenyl)silanol (1ab): Following the general procedure, 1ab was synthesized from commercial available S2 as slightly yellow oil (1.8 g, 94\% for two steps).

$\mathrm{R} f=0.5$ (petroleum ether/ethyl acetate $=12: 1$ ); IR (film): 3600, 1586, 1422, 1106, 813, 745, 710, 507 $\mathrm{cm}^{-1} ;{ }^{1} \mathrm{H}$ NMR $\left(400 \mathrm{MHz}, \mathrm{CDCl}_{3}\right) \delta 7.60(\mathrm{~d}, J=7.5 \mathrm{~Hz}, 1 \mathrm{H}), 7.35(\mathrm{t}, J=7.5 \mathrm{~Hz}, 1 \mathrm{H}), 7.28(\mathrm{t}, J=7.5$ $\mathrm{Hz}, 1 \mathrm{H}), 7.17$ (d, J=7.5 Hz, 1H), 5.22-5.19 (m, 1H), 4.93-4.91 (m, 1H), 2.14 (brs, 1H), 2.13 (brs, 3H), $0.41(\mathrm{~s}, 6 \mathrm{H}) .{ }^{13} \mathrm{C} \mathrm{NMR}\left(126 \mathrm{MHz} \mathrm{CDCl}_{3}\right) \delta 150.00,149.08,136.32,134.32,129.09,127.25,126.26$, 115.31, 25.72, 1.84. HRMS-DART Positive calcd. for $\mathrm{C}_{11} \mathrm{H}_{17} \mathrm{OSi}[\mathrm{M}+\mathrm{H}]^{+}$193.1049, found: 193.1050.

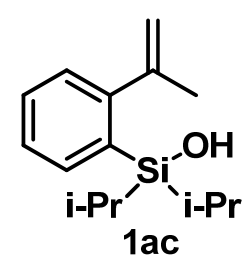

Diisopropyl(2-(prop-1-en-2-yl)phenyl)silanol (1ac): Following the general procedure, 1ac was synthesized from commercial available S2 as slightly yellow oil (2.2 g, 89\% for two steps). 
$\mathrm{R} f=0.5$ (petroleum ether/ethyl acetate $=12: 1$ ); IR (film): 3605, 1582, 1428, 1109, 815, 740, 718, 502

$\mathrm{cm}^{-1} ;{ }^{1} \mathrm{H}$ NMR $\left(400 \mathrm{MHz}, \mathrm{CDCl}_{3}\right)$ 8 7.45-7.39 (m, 1H), 7.34-7.29 (m, 1H), 7.27-7.22 (m, 1H),

7.13-7.09 (m, 1H), 5.20-5.17 (m, 1H), 4.90-4.87 (m, 1H), 2.50 (brs, 1H), 2.14 (brs, 1H), 2.13 (s, 3H),

$1.23-1.13(\mathrm{~m}, 2 \mathrm{H}), 1.05(\mathrm{~d}, J=7.0 \mathrm{~Hz}, 6 \mathrm{H}), 0.96(\mathrm{~d}, J=7.0 \mathrm{~Hz}, 6 \mathrm{H}) \cdot{ }^{13} \mathrm{C}$ NMR $\left(126 \mathrm{MHz}, \mathrm{CDCl}_{3}\right) \delta$

151.09, 150.48, 134.22, 132.78, 128.60, 127.50, 125.81, 113.99, 26.36, 17.65, 17.25, 13.75 .

HRMS-DART Positive calcd. for $\mathrm{C}_{15} \mathrm{H}_{25} \mathrm{OSi}[\mathrm{M}+\mathrm{H}]^{+} 249.1675$, found: 249.1673 .<smiles>C=C(C)c1cc(C)ccc1[Si](O)(c1ccccc1)c1ccccc1</smiles>

1b

Diphenyl(4-methyl-2-(prop-1-en-2-yl)phenyl)silanol (1b): Following the general procedure, $1 \mathbf{b}$ was synthesized from commercial available S1 as slightly yellow oil ( $2.3 \mathrm{~g}, 70 \%$ for three steps). $\mathrm{R} f=0.5$ (petroleum ether/ethyl acetate $=10: 1)$; IR (film): 3605, 2967, 1596, 1427, 1119, 810, 745, 701, $508 \mathrm{~cm}^{-1} ;{ }^{1} \mathrm{H}$ NMR $\left(400 \mathrm{MHz}, \mathrm{CDCl}_{3}\right) \delta$ 7.6-7.57 (m, 4H), 7.44-7.31 (m, 7H), 7.09-7.03 (m, 2H), 5.08-5.04 (m, 1H), 4.84-4.80 (m, 1H), 2.91 (brs, $1 \mathrm{H}), 2.37$ (s, 3H), $1.91(\mathrm{~s}, 3 \mathrm{H}) .{ }^{13} \mathrm{C}$ NMR $(126 \mathrm{MHz}$, $\left.\mathrm{CDCl}_{3}\right) \delta 150.87,149.69,139.72,136.87,136.61,135.02,129.76,128.91,128.37,127.75,127.00$, 115.47, 25.31, 21.47. HRMS-DART Positive calcd. for $\mathrm{C}_{22} \mathrm{H}_{23} \mathrm{OSi}[\mathrm{M}+\mathrm{H}]^{+} 331.1518$, found: 331.1512 .<smiles>C=C(C)c1ccc(C)cc1[Si](O)(c1ccccc1)c1ccccc1</smiles>

Diphenyl(5-methyl-2-(prop-1-en-2-yl)phenyl)silanol (1c): Following the general procedure, 1c was synthesized from commercial available S1 as slightly yellow oil (2.4 g, $73 \%$ for three steps).

$\mathrm{R} f=0.5$ (petroleum ether/ethyl acetate $=10: 1$ ); IR (film): 3603, 2967, 1594, 1426, 1118, 808, 747, 700, $506 \mathrm{~cm}^{-1}$; ${ }^{1} \mathrm{H}$ NMR $\left(400 \mathrm{MHz}, \mathrm{CDCl}_{3}\right)$ 8 7.63-7.60 (m, 4H), 7.46-7.37 (m, 6H), 7.27-7.23 (m, 2H), 7.15-7.12 (m, 1H), 5.07-5.06 (m, 1H), 4.83-4.82 (m, 1H), 2.95 (brs, $1 \mathrm{H}), 2.30(\mathrm{~s}, 3 \mathrm{H}), 1.91(\mathrm{~s}, 3 \mathrm{H}) .{ }^{13} \mathrm{C}$ $\operatorname{NMR}\left(126 \mathrm{MHz}, \mathrm{CDCl}_{3}\right) \delta 149.60,147.95,137.11,136.49,135.50,135.06,132.18,130.49,129.78$, 127.75, 127.50, 115.56, 25.40, 21.23. HRMS-DART Positive calcd. for $\mathrm{C}_{22} \mathrm{H}_{23} \mathrm{OSi}[\mathrm{M}+\mathrm{H}]^{+} 331.1518$, found: 331.1517 .<smiles>C=C(C)c1cc(OC)ccc1[Si](O)(c1ccccc1)c1ccccc1</smiles>

Diphenyl(4-methoxyl-2-(prop-1-en-2-yl)phenyl)silanol (1d): Following the general procedure, 1d was synthesized from commercial available S1 as slightly yellow oil ( $2.6 \mathrm{~g}, 75 \%$ for three steps).

$\mathrm{R} f=0.5$ (petroleum ether/ethyl acetate $=6: 1$ ); IR (film): 3605, 1596, 1427, 1118, 807, 748, 702, 508 $\mathrm{cm}^{-1}$; ${ }^{1} \mathrm{H}$ NMR $\left(400 \mathrm{MHz}, \mathrm{CDCl}_{3}\right) \delta$ 7.59-7.56 (m, 4H), 7.43-7.32 (m, 7H), 6.79-6.75 (m, 2H), 5.06-5.02 (m, 1H), 4.84-4.82 (m, 1H), $3.81(\mathrm{~s}, 3 \mathrm{H}), 2.81$ (brs, $1 \mathrm{H}), 1.87$ (s, 3H). ${ }^{13} \mathrm{C}$ NMR $(126 \mathrm{MHz}$, $\left.\mathrm{CDCl}_{3}\right) \delta 160.73,152.71,149.18,138.46,136.71,134.99,129.72,127.73,123.63,115.59,113.61$, 111.56, 55.06, 25.17. HRMS-DART Positive calcd. for $\mathrm{C}_{22} \mathrm{H}_{23} \mathrm{O}_{2} \mathrm{Si}[\mathrm{M}+\mathrm{H}]^{+}$347.1467, found: 347.1470 . 
<smiles>[CH][P+](C)(c1ccccc1)[Si](O)(c1ccccc1)c1ccc(Cl)cc1C(=C)C</smiles>

Diphenyl(4-chloro-2-(prop-1-en-2-yl)phenyl)silanol (1e): Following the general procedure, 1e was synthesized from commercial available S1 as slightly yellow oil ( $2.1 \mathrm{~g}, 60 \%$ for three steps).

$\mathrm{R} f=0.5$ (petroleum ether/ethyl acetate =12:1); IR (film): 3608, 1594, 1426, 1117, 809, 744, 701, 505 $\mathrm{cm}^{-1} ;{ }^{1} \mathrm{H}$ NMR $\left(400 \mathrm{MHz}, \mathrm{CDCl}_{3}\right) \delta$ 7.85-7.52 (m, 4H), 7.45-7.32 (m, 7H), 7.22-7.17 (m, 2H), 5.08-5.04 (m, 1H), 4.82-4.79 (m, 1H), 2.82 (brs, 1H), $1.85(\mathrm{~s}, 3 \mathrm{H}) .{ }^{13} \mathrm{C} \mathrm{NMR}\left(126 \mathrm{MHz}, \mathrm{CDCl}_{3}\right) \delta$ $152.56,148.07,138.11,136.03,135.85,134.95,131.03,130.02,127.87,127.74,126.29,116.48,25.00$. HRMS-DART Positive calcd. for $\mathrm{C}_{21} \mathrm{H}_{20} \mathrm{ClOSi}[\mathrm{M}+\mathrm{H}]^{+}$351.0972, found: 351.0970 .<smiles>C=C(C)c1ccc(Cl)cc1[Si](O)(c1ccccc1)c1ccccc1</smiles>

$1 f$

Diphenyl(5-chloro-2-(prop-1-en-2-yl)phenyl)silanol (1f): Following the general procedure, 1f was synthesized from commercial available S1 as slightly yellow oil (1.9 g, 54\% for three steps).

$\mathrm{R} f=0.5$ (petroleum ether/ethyl acetate $=12: 1$ ); IR (film): 3605, 1596, 1427, 1118, 808, 746, 700, 504 $\mathrm{cm}^{-1} ;{ }^{1} \mathrm{H}$ NMR $\left(400 \mathrm{MHz}, \mathrm{CDCl}_{3}\right) \delta$ 7.59-7.53 (m, 4H), 7.46-7.33 (m, 8H), 7.14-7.10 (m, 1H), 5.06-5.02 (m, 1H), 4.79-4.76 (m, 1H), 2.84 (brs, 1H), $1.82(\mathrm{~s}, 3 \mathrm{H}) .{ }^{13} \mathrm{C} \mathrm{NMR}\left(126 \mathrm{MHz}, \mathrm{CDCl}_{3}\right) \delta$ 149.06, 148.30, 136.02, 135.49, 135.31, 134.97, 132.51, 130.10, 129.77, 129.14, 127.90, 116.37, 25.14. HRMS-DART Positive calcd. for $\mathrm{C}_{21} \mathrm{H}_{20} \mathrm{ClOSi}[\mathrm{M}+\mathrm{H}]^{+}$351.0972, found: 351.0975.<smiles>C=C(C)c1cc(C(F)(F)F)ccc1[Si](O)(c1ccccc1)c1ccccc1</smiles>

$1 \mathrm{~g}$

Diphenyl(5-trifluoromethyl-2-(prop-1-en-2-yl)phenyl)silanol (1g): Following the general procedure, $1 \mathrm{~g}$ was synthesized from commercial available S1 as slightly yellow oil (1.6 g, 41\% for three steps). $\mathrm{R} f=0.5$ (petroleum ether/ethyl acetate =15:1); IR (film): 3602, 1596, 1425, 1118, 806, 745, 706, 502 $\mathrm{cm}^{-1} ;{ }^{1} \mathrm{H}$ NMR $\left(400 \mathrm{MHz}, \mathrm{CDCl}_{3}\right) \delta$ 7.59-7.53 (m, 5H), 7.46-7.35 (m, 8H), 5.12-5.08 (m, 1H), 4.85-4.81 (m, 1H), 2.88 (brs, 1H), $1.88(\mathrm{~s}, 3 \mathrm{H}) .{ }^{19} \mathrm{~F} \mathrm{NMR}\left(376 \mathrm{MHz}, \mathrm{CDCl}_{3}\right) \delta-63.00 .{ }^{13} \mathrm{C} \mathrm{NMR}(126$ $\left.\mathrm{MHz}, \mathrm{CDCl}_{3}\right) \delta 151.46,148.15,137.45,137.18,135.43,134.95,131.66$ (d, J= 7.6 Hz), 130.17, 127.94, $124.08(\mathrm{q}, J=3.8 \mathrm{~Hz}), 122.55(\mathrm{q}, J=3.8 \mathrm{~Hz}), 116.85,25.05$. HRMS-DART Positive calcd. for $\mathrm{C}_{22} \mathrm{H}_{20} \mathrm{~F}_{3} \mathrm{OSi}[\mathrm{M}+\mathrm{H}]^{+}$385.1236, found: 385.1234 .<smiles>C=C(C)c1cccc(C)c1[Si](O)(c1ccccc1)c1ccccc1</smiles>

$1 \mathrm{~h}$

Diphenyl(6-methyl-2-(prop-1-en-2-yl)phenyl)silanol (1h): Following the general procedure, 1h was 
synthesized from commercial available S1 as slightly yellow oil (1.0 g, 30\% for three steps).

$\mathrm{R} f=0.5$ (petroleum ether/ethyl acetate $=10: 1)$; IR (film): 3604, 2967, 1598, 1424, 1112, 814, 746, 700, $507 \mathrm{~cm}^{-1}$; ${ }^{1} \mathrm{H}$ NMR (400 MHz, $\left.\mathrm{CDCl}_{3}\right)$ 8 7.65-7.59 (m, 4H), 7.44-7.30 (m, 7H), 7.09-7.04 (m, 2H), 5.07-5.05 (m, 1H), 4.83-4.81 (m, 1H), 3.24 (brs, 1H), 2.11 (s, 3H), 2.06 (s, 3H). ${ }^{13} \mathrm{C}$ NMR (126 MHz, $\left.\mathrm{CDCl}_{3}\right) \delta 151.98,151.42,144.98,137.01,135.04,130.59,129.76,129.59,129.21,127.87,125.30$, 115.48, 26.08, 25.00. HRMS-DART Positive calcd. for $\mathrm{C}_{22} \mathrm{H}_{23} \mathrm{OSi}[\mathrm{M}+\mathrm{H}]^{+} 331.1518$, found: 331.1516.<smiles>C=C(C)c1ccc2ccccc2c1[Si](O)(c1ccccc1)c1ccccc1</smiles>

Diphenyl(2-(prop-1-en-2-yl)naphthalen-1-yl)silanol (1i): Following the general procedure, 1i was synthesized from commercial available S1 as slightly yellow oil (1.2 g, 32\% for three steps). $\mathrm{R} f=0.5$ (petroleum ether/ethyl acetate $=8: 1$ ); IR (film): 3603, 1597, 1425, 1120, 811, 745, 707, 502 $\mathrm{cm}^{-1} ;{ }^{1} \mathrm{H}$ NMR $\left(500 \mathrm{MHz}, \mathrm{CDCl}_{3}\right) \delta 8.00(\mathrm{~d}, J=8.5 \mathrm{~Hz}, 1 \mathrm{H}), 7.93(\mathrm{~d}, J=8.5 \mathrm{~Hz}, 1 \mathrm{H}), 7.84(\mathrm{~d}, J=8.0$ $\mathrm{Hz}, 1 \mathrm{H}), 7.71-7.65(\mathrm{~m}, 4 \mathrm{H}), 7.45-7.33(\mathrm{~m}, 8 \mathrm{H}), 7.21-7.17(\mathrm{~m}, 1 \mathrm{H}), 5.12-5.09(\mathrm{~m}, 1 \mathrm{H}), 4.93-4.91(\mathrm{~m}$, 1H), 3.29 (brs, 1H), 2.17 (s, 3H). $\left.{ }^{13} \mathrm{C} \mathrm{NMR} \mathrm{(126} \mathrm{MHz,} \mathrm{CDCl}_{3}\right) \delta 151.54,151.05,137.61,137.32$, $135.15,132.30,130.51,129.81,129.65,128.66,128.46,127.87,126.06,125.57,125.30,116.74,25.35$. HRMS-DART Positive calcd. for $\mathrm{C}_{25} \mathrm{H}_{23} \mathrm{OSi}[\mathrm{M}+\mathrm{H}]^{+} 367.1518$, found: 367.1513 .<smiles>C=C(CC)c1ccccc1[Si](O)(c1ccccc1)c1ccccc1</smiles>

Diphenyl(2-(but-1-en-2-yl)phenyl)silanol (1j): Following the general procedure, $\mathbf{1 j}$ was synthesized from commercial available S1 as slightly yellow oil ( $2.5 \mathrm{~g}, 76 \%$ for three steps). $\mathrm{R} f=0.5$ (petroleum ether/ethyl acetate $=15: 1$ ); IR (film): 3606, 2968, 2821, 1595, 1425, 1122, 810, 748, 703, $506 \mathrm{~cm}^{-1}$; ${ }^{1} \mathrm{H}$ NMR $\left(500 \mathrm{MHz}, \mathrm{CDCl}_{3}\right) \delta$ 7.63-7.57 (m, 4H), 7.46-7.35 (m, 8H), 7.26-7.22 (m, $1 \mathrm{H}), 7.20-7.17(\mathrm{~m}, 1 \mathrm{H}), 5.09-5.07(\mathrm{~m}, 1 \mathrm{H}), 4.91-4.89(\mathrm{~m}, 1 \mathrm{H}), 2.91$ (brs, 1H), 2.27-2.21 (m, 2H), 0.96 $(\mathrm{t}, J=7.5 \mathrm{~Hz}, 3 \mathrm{H}) .{ }^{13} \mathrm{C}$ NMR $\left(126 \mathrm{MHz}, \mathrm{CDCl}_{3}\right) \delta 155.37,150.54,136.66,136.42,134.99,132.65$, 129.81, 129.51, 127.86, 127.76, 126.03, 112.96, 31.13, 11.84. HRMS-DART Positive calcd. for $\mathrm{C}_{22} \mathrm{H}_{23} \mathrm{OSi}[\mathrm{M}+\mathrm{H}]^{+} 331.1518$, found: 367.1514 .

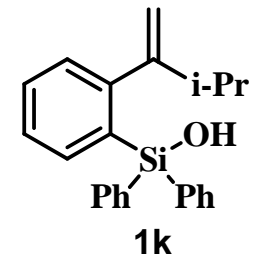

Diphenyl(2-(3-methylbut-1-en-2-yl)phenyl)silanol (1k): Following the general procedure, $1 \mathbf{k}$ was synthesized from commercial available $\mathbf{S 1}$ as slightly yellow oil (2.5 g, $73 \%$ for three steps).

$\mathrm{R} f=0.5$ (petroleum ether/ethyl acetate $=15: 1$ ); IR (film): 3602, 2967, 2826, 1592, 1424, 1128, 811, 744, 709, $501 \mathrm{~cm}^{-1} ;{ }^{1} \mathrm{H}$ NMR (500 MHz, $\left.\mathrm{CDCl}_{3}\right) \delta$ 7.62-7.58 (m, 4H), 7.46-7.36 (m, 8H), 7.27-7.21 (m, $2 \mathrm{H}), 5.16-5.15(\mathrm{~m}, 1 \mathrm{H}), 4.96-4.95(\mathrm{~m}, 1 \mathrm{H}), 3.00(\mathrm{brs}, 1 \mathrm{H}), 2.64-2.56(\mathrm{~m}, 1 \mathrm{H}), 1.06(\mathrm{~d}, J=7.0 \mathrm{~Hz}, 6 \mathrm{H})$. ${ }^{13} \mathrm{C}$ NMR $\left(126 \mathrm{MHz}, \mathrm{CDCl}_{3}\right) \delta 160.17,150.26,136.70,136.59,135.02,132.72,129.83,129.30,128.33$, 127.79, 125.99, 111.89, 34.89, 21.38. HRMS-DART Positive calcd. for $\mathrm{C}_{23} \mathrm{H}_{25} \mathrm{OSi}[\mathrm{M}+\mathrm{H}]^{+} 345.1675$, 
found: 345.1670 .<smiles>CC(C)=Cc1ccccc1[Si](O)(c1ccccc1)c1ccccc1</smiles>

Diphenyl(2-(2-methylprop-1-enyl)phenyl)silanol (1'l): Following the general procedure, 1'l was synthesized from commercial available S2 as slightly yellow oil (3.0 g, 91\% for two steps).

$\mathrm{R} f=0.5$ (petroleum ether/ethyl acetate =12:1); IR (film): 3603, 1595, 1420, 1122, 810, 740, 703, 508 $\mathrm{cm}^{-1} ;{ }^{1} \mathrm{H}$ NMR $\left(400 \mathrm{MHz}, \mathrm{CDCl}_{3}\right) \delta$ 7.62-7.54 (m, 4H), 7.50-7.33 (m, 8H), 7.22-7.14 (m, 2H), $6.24(\mathrm{~s}$, 1H), 2.59 (brs, 1H), 1.63 (s, 3H), $1.53(\mathrm{~s}, 3 \mathrm{H}) .{ }^{13} \mathrm{C} \mathrm{NMR}\left(126 \mathrm{MHz}, \mathrm{CDCl}_{3}\right) \delta 145.24,136.77,136.01$, 135.69, 134.93, 134.11, 129.90, 129.82, 129.64, 127.76, 126.66, 125.47, 25.65, 19.11. HRMS-DART Positive calcd. for $\mathrm{C}_{22} \mathrm{H}_{23} \mathrm{OSi}[\mathrm{M}+\mathrm{H}]^{+} 331.1518$, found: 331.1515 .<smiles>O[Si](c1ccccc1)(c1ccccc1)c1ccccc1/C=C\c1ccccc1</smiles>

Diphenyl(2-(Z)-styrylphenyl)silanol (1'm): Following the general procedure, 1'm was synthesized from commercial available S2 as slightly yellow oil ( $2.5 \mathrm{~g}, 66 \%$ for two steps).

Note: For the synthesis of S3, the pre-prepared solution of Wittig reagent and t-BuOK was cooled to $-30{ }^{\circ} \mathrm{C}$, and then the solution of $\mathbf{S} 2$ in $10 \mathrm{~mL}$ THF was added. The reaction was stirred at the same temperature for $48 \mathrm{~h}$, at which time TLC (petroleum ether/ethyl acetate $=8: 1$ ) showed the starting material was consumed completely, and then the reaction was quenched by pouring into saturate aq. $\mathrm{NH}_{4} \mathrm{Cl}$. NMR of the crude S3 showed only one isomer of double bond formed in the reaction, and the configuration of double bond was determined to be Z- by NOESY spectrum.

$\mathrm{R} f=0.5$ (petroleum ether/ethyl acetate $=8: 1$ ); IR (film): 3604, 1597, 1421, 1128, 807, 742, 705, 502 $\mathrm{cm}^{-1} ;{ }^{1} \mathrm{H}$ NMR $\left(400 \mathrm{MHz}, \mathrm{CDCl}_{3}\right) \delta$ 7.67-7.59 (m, 4H), 7.51-7.47 (m, 1H), 7.45-7.33 (m, 6H), 7.30-7.19 (m, 3H), 7.17-7.12 (m, 3H), 7.09-7.04 (m, 2H), 6.67 (d, $J=12.0 \mathrm{~Hz}, 1 \mathrm{H}), 6.43(\mathrm{~d}, J=12.0$ $\mathrm{Hz}, 1 \mathrm{H}), 2.63$ (brs, 1H). ${ }^{13} \mathrm{C}$ NMR $\left(126 \mathrm{MHz} \mathrm{CDCl}_{3}\right) \delta 144.26,136.74,136.36,135.37,134.93,134.14$, $131.73,131.09,130.20,130.02,129.34,129.15,128.13,127.90,127.27,126.38$. HRMS-DART Positive calcd. for $\mathrm{C}_{26} \mathrm{H}_{23} \mathrm{OSi}[\mathrm{M}+\mathrm{H}]^{+} 379.1518$, found: 379.1520 .<smiles>C/C=C(/C)c1ccccc1[Si](O)(c1ccccc1)c1ccccc1</smiles>

Diphenyl(2-((E)-but-2-en-2-yl)phenyl)silanol (1n): Following the general procedure, 1n was synthesized from commercial available $\mathbf{S} 2$ as colorless oil after purification by preparative HPLC (1.7 g, $51 \%$ for two steps). 
Note: For the synthesis of S3, the solvent used was toluene, after the pre-prepared solution of Wittig reagent and $t$-BuOK was heated to $130{ }^{\circ} \mathrm{C}$, the solution of $\mathbf{S} 2$ in $10 \mathrm{~mL}$ toluene was added dropwise carefully. The reaction was stirred at the same temperature for $6 \mathrm{~h}$. After the reaction was cooled to room temperature, it was purified following the general procedure. NMR of the crude S3 showed two isomers of double bond (The ratio was 3:1) formed in the reaction, and the crude S3 was used in the next step directly. And NMR of the product got from the next step showed it was a mixture of two isomers of double bond (The ratio was 3:1). The product was purified by preparative HPLC to afford the major isomer, whose exact configuration of double bond was confirmed to be $E$ by NOESY spectrum.

$\mathrm{R} f=0.5$ (petroleum ether/ethyl acetate $=10: 1$ ); IR (film): 3603, 1596, 1420, 1129, 803, 745, 709, 503 $\mathrm{cm}^{-1}$; ${ }^{1} \mathrm{H}$ NMR $\left(400 \mathrm{MHz}, \mathrm{CDCl}_{3}\right) \delta$ 7.61-7.55 (m, 4H), 7.47-7.33 (m, 8H), 7.23-7.13 (m, 2H), 5.32-5.24 (m, 1H), 2.68 (brs, $1 \mathrm{H}), 1.67(\mathrm{~s}, 3 \mathrm{H}), 1.52(\mathrm{~d}, J=7.0,3 \mathrm{H}) .{ }^{13} \mathrm{C}$ NMR $\left(126 \mathrm{MHz}, \mathrm{CDCl}_{3}\right) \delta$ 152.92, 139.76, 136.72, 136.53, 134.80, 132.81, 129.69, 129.66, 127.93, 127.70, 125.64, 125.01, 18.34, 13.65. HRMS-DART Positive calcd. for $\mathrm{C}_{22} \mathrm{H}_{22} \mathrm{OSi}[\mathrm{M}+\mathrm{H}]^{+} 331.1518$, found: 331.1516 .<smiles>C/C=C(\C)c1ccccc1[Si](O)(c1ccccc1)c1ccccc1</smiles>

1q

Diphenyl(2-((Z)-but-2-en-2-yl)phenyl)silanol (1q): Following the general procedure, 1q was synthesized from commercial available S2 as slightly yellow oil (2.8 g, 85\% for two steps).

Note: For the synthesis of S3, the pre-prepared solution of Wittig reagent and $t$-BuOK was cooled to $-35{ }^{\circ} \mathrm{C}$, and then the solution of $\mathbf{S 2}$ in $10 \mathrm{~mL}$ THF was added. The reaction was stirred at the same temperature for $72 \mathrm{~h}$, at which time TLC (petroleum ether/ethyl acetate $=8: 1$ ) showed the starting material was consumed completely, and then the reaction was quenched by pouring into saturate aq. $\mathrm{NH}_{4} \mathrm{Cl}$. NMR of the crude $\mathbf{S} 3$ showed only one isomer of double bond formed in the reaction, and the exact configuration of double bond was confirmed to be $Z$ - by NOESY.

$\mathrm{R} f=0.5$ (petroleum ether/ethyl acetate $=10: 1$ ); IR (film): 3605, 1597, 1423, 1126, 808, 740, 702, 506 $\mathrm{cm}^{-1}$; ${ }^{1} \mathrm{H}$ NMR $\left(500 \mathrm{MHz}, \mathrm{CDCl}_{3}\right) \delta$ 7.70-7.63 (m, 4H), 7.53-7.39 (m, 8H), 7.31-7.26 (m, 1H), 7.13-7.09 (m, 1H), 5.66-5.60 (m, 1H), 3.24 (brs, $1 \mathrm{H}), 1.91$ (s, 3H), 1.34 (d, $J=6.7 \mathrm{~Hz}, 3 \mathrm{H}) .{ }^{13} \mathrm{C}$ NMR $\left(126 \mathrm{MHz}, \mathrm{CDCl}_{3}\right) \delta 149.04,141.27,136.57,135.18,135.03,132.70,130.17,129.92,128.52,127.79$, 125.78, 123.14, 26.70, 15.00. HRMS-DART Positive calcd. for $\mathrm{C}_{22} \mathrm{H}_{22} \mathrm{OSi}[\mathrm{M}+\mathrm{H}]^{+}$331.1518, found: 331.1514 .<smiles>CCC/C=C(\C)c1ccccc1[Si](O)(c1ccccc1)c1ccccc1</smiles>

Diphenyl(2-((E)-hex-2-en-2-yl)phenyl)silanol (10): Following the general procedure, 10 was synthesized from commercial available S2 as slightly yellow oil (3.2 g, 86\% for two steps).

Note: For the synthesis of S3, the solvent used was toluene, after the pre-prepared solution of Wittig 
reagent and $t$-BuOK was heated to $130{ }^{\circ} \mathrm{C}$, the solution of $\mathbf{S 2}$ in $10 \mathrm{~mL}$ toluene was added dropwise carefully(Note: The Wittig reagent used was tetrabutylphosphonium bromide.). The reaction was stirred at the same temperature for $6 \mathrm{~h}$. After the reaction was cooled to room temperature, it was purified following the general procedure. NMR of the crude S3 showed only one isomer of double bond formed in the reaction, and the exact configuration of double bond was confirmed to be $E$ by NOESY.

$\mathrm{R} f=0.5$ (petroleum ether/ethyl acetate $=20: 1)$; IR (film): 3601, 2964, 2877, 2826, 1594, 1423, 1126, 810, 743, 705, $509 \mathrm{~cm}^{-1}$; ${ }^{1} \mathrm{H}$ NMR $\left(400 \mathrm{MHz}, \mathrm{CDCl}_{3}\right) \delta$ 7.61-7.54 (m, 4H), 7.45-7.33 (m, 8H), 7.23-7.14 (m, 2H), 5.28-5.22 (m, 1H), $2.72($ brs, $1 \mathrm{H}), 1.93$ (q, $J=7.5 \mathrm{~Hz}, 2 \mathrm{H}), 1.72(\mathrm{~s}, 3 \mathrm{H}), 1.29-1.28$ $(\mathrm{m}, 2 \mathrm{H}), 0.82(\mathrm{t}, J=7.5 \mathrm{~Hz}, 3 \mathrm{H}) .{ }^{13} \mathrm{C}$ NMR $\left(126 \mathrm{MHz}, \mathrm{CDCl}_{3}\right) \delta 152.85,139.15,136.72,136.54$, 134.88, 132.64, 130.83, 129.69, 129.66, 128.06, 127.70, 125.61, 30.34, 22.36, 18.76, 13.90. HRMS-DART Positive calcd. for $\mathrm{C}_{25} \mathrm{H}_{29} \mathrm{OSi}[\mathrm{M}+\mathrm{H}]^{+} 373.1988$, found: 373.1985 .<smiles>CCC/C=C(/C)c1ccccc1[Si](O)(c1ccccc1)c1ccccc1</smiles>

Diphenyl(2-((Z)-hex-2-en-2-yl)phenyl)silanol (1r): Following the general procedure, 1r was synthesized from commercial available S2 as slightly yellow oil (3.2 g, 90\% for two steps).

Note: For the synthesis of S3, the pre-prepared solution of Wittig reagent and $t$-BuOK was cooled to $-15{ }^{\circ} \mathrm{C}$, and then the solution of $\mathbf{S} 2 \mathrm{in} 10 \mathrm{~mL}$ THF was added. The reaction was stirred at the same temperature for $96 \mathrm{~h}$, at which time TLC (petroleum ether/ethyl acetate $=8: 1$ ) showed the starting material was consumed completely, and then the reaction was quenched by pouring into saturate aq. $\mathrm{NH}_{4} \mathrm{Cl}$. NMR of the crude $\mathbf{S} 3$ showed only one isomer of double bond formed in the reaction, and the exact configuration of double bond was confirmed to be Z- by NOESY.

$\mathrm{R} f=0.5$ (petroleum ether/ethyl acetate $=20: 1$ ); IR (film): 3605, 2966, 2932, 2860, 1593, 1420, 1123 , 806, 743, 712, $516 \mathrm{~cm}^{-1}$; ${ }^{1} \mathrm{H}$ NMR (400 MHz, $\left.\mathrm{CDCl}_{3}\right)$ 87.67-7.60 (m, 4H), 7.51-7.35 (m, 8H), 7.29-7.23 (m, 1H), 7.12-7.06 (m, 1H), 5.57 (t, $J=6.5 \mathrm{~Hz}, 1 \mathrm{H}), 3.27$ (brs, 1H), 1.91 (s, 3H), 1.80-1.61 (m, 2H), 1.34-1.22 (m, 2H), $0.79(\mathrm{t}, J=7.0 \mathrm{~Hz}, 3 \mathrm{H}) .{ }^{13} \mathrm{C} \mathrm{NMR}\left(126 \mathrm{MHz}, \mathrm{CDCl}_{3}\right) \delta 149.14,140.44$, 136.49, 136.29, 135.85, 135.21, 134.97, 132.50, 130.02, 129.91, 129.08, 128.58, 127.78, 125.72, 31.47, 26.83, 22.74, 13.84. HRMS-DART Positive calcd. for $\mathrm{C}_{24} \mathrm{H}_{27} \mathrm{OSi}[\mathrm{M}+\mathrm{H}]^{+} 359.1831$, found: 359.1831 .<smiles>C/C(=C\c1ccccc1)c1ccccc1[Si](O)(c1ccccc1)c1ccccc1</smiles><smiles>C/C=C\c1ccccc1[Si](O)(c1ccccc1)c1ccccc1</smiles>

Following the general procedure, $\mathbf{1 p}$ and $\mathbf{1 s}$ were obtained from commercial available S2 as an inseparable mixture by flash chromatography (The ratio was $1: 1$.), as slightly yellow oil. Purification by preparative TLC afforded pure $\mathbf{1 p}(0.7 \mathrm{~g}, 18 \%$ for two steps) and pure $1 \mathrm{~s}(0.7 \mathrm{~g}, 18 \%$ for two steps). And the exact configuration of double bong was confirmed by NOESY. 
Diphenyl(2-((E)-1-phenylprop-1-en-2-yl)phenyl)silanol (1p): $\mathrm{R} f=0.50$ (petroleum ether/ethyl acetate =7:1); IR (film): 3605, 1596, 1424, 1129, 808, 745, 706, $502 \mathrm{~cm}^{-1} ;{ }^{1} \mathrm{H}$ NMR $\left(500 \mathrm{MHz}, \mathrm{CDCl}_{3}\right)$ $\delta$ 7.63-7.58 (m, 4H), 7.56-7.52 (m, 1H), 7.48-7.41 (m, 3H), 7.39-7.35 (m, 4H), 7.33-7.27 (m, 4H), 7.24-7.19 (m, 1H), 7.00-6.97 (m, 2H), 6.33-6.31 (m, 1H), $2.62(\mathrm{brs}, 1 \mathrm{H}), 1.97(\mathrm{~d}, J=1.5 \mathrm{~Hz}, 3 \mathrm{H}) .{ }^{13} \mathrm{C}$ NMR (126 MHz, $\left.\mathrm{CDCl}_{3}\right) \delta 153.13,141.52,137.06,137.04,136.42,134.91,132.95,130.16,129.85$, $129.78,128.85,128.12,127.84,127.78,126.63,125.99,20.53$. HRMS-DART Positive calcd. for $\mathrm{C}_{27} \mathrm{H}_{25} \mathrm{OSi}[\mathrm{M}+\mathrm{H}]^{+}$393.1675, found: 393.1678 .

Diphenyl(2-((Z)-1-phenylprop-1-en-2-yl)phenyl)silanol (1s): $\mathrm{R} f=0.52$ (petroleum ether/ethyl acetate = 7:1); IR (film): 3603, 1595, 1420, 1122, 810, 747, 709, $505 \mathrm{~cm}^{-1} ;{ }^{1} \mathrm{H} \mathrm{NMR}\left(500 \mathrm{MHz}, \mathrm{CDCl}_{3}\right)$ $\delta$ 7.65-7.59 (m, $2 \mathrm{H}), 7.55-7.47(\mathrm{~m}, 4 \mathrm{H}), 7.46-7.41(\mathrm{~m}, 1 \mathrm{H}), 7.41-7.36(\mathrm{~m}, 3 \mathrm{H}), 7.33-7.28(\mathrm{~m}, 3 \mathrm{H})$, 7.26-7.22 (m, 1H), 7.15-7.09 (m, 3H), 6.92-6.87 (m, 2H), 6.41-6.39 (m, 1H), 2.62 (brs, $1 \mathrm{H}), 1.89$ (d, $J$ $=1.5 \mathrm{~Hz}, 3 \mathrm{H}) .{ }^{13} \mathrm{C} \mathrm{NMR}\left(126 \mathrm{MHz}, \mathrm{CDCl}_{3}\right) \delta 149.28,141.94,137.60,136.48,136.23,135.66,134.98$, $134.70,132.44,130.82,129.75,129.69,128.64,128.49,128.35,127.68,127.66,127.62,126.88$, 126.21, 28.57. HRMS-DART Positive calcd. for $\mathrm{C}_{27} \mathrm{H}_{25} \mathrm{OSi}[\mathrm{M}+\mathrm{H}]^{+}$393.1675, found: 393.1676 .<smiles>CC(C)=C(C)c1ccccc1[Si](O)(c1ccccc1)c1ccccc1</smiles>

Diphenyl(2-(3-methylbut-2-en-2-yl)phenyl)silanol (1t): Following the general procedure, 1t was synthesized from commercial available S2 as slightly yellow oil (1.5 g, 43\% for two steps).

Note: For the synthesis of S3, the solvent used was toluene, after the pre-prepared solution of Wittig reagent and t-BuOK was heated to $130{ }^{\circ} \mathrm{C}$, the solution of $\mathbf{S} 2$ in $10 \mathrm{~mL}$ toluene was added dropwise carefully(Note: The Wittig reagent used was isopropyl triphenylphosphonium iodide.). The reaction was stirred at the same temperature for $12 \mathrm{~h}$. After the reaction was cooled to room temperature, it was purified following the general procedure.

$\mathrm{R} f=0.5$ (petroleum ether/ethyl acetate $=12: 1$ ); IR (film): 3605, 1596, 1423, 1124, 806, 742, 705, 508 $\mathrm{cm}^{-1}$; ${ }^{1} \mathrm{H}$ NMR $\left(500 \mathrm{MHz}, \mathrm{CDCl}_{3}\right)$ 87.66-7.63 (m, 4H), 7.54-7.50 (m, $\left.1 \mathrm{H}\right), 7.48-7.38(\mathrm{~m}, 7 \mathrm{H})$, 7.28-7.22 (m, 1H), 7.08-7.06 (m, 1H), $3.04(\mathrm{brs}, 1 \mathrm{H}), 1.78(\mathrm{~s}, 3 \mathrm{H}), 1.76(\mathrm{~s}, 3 \mathrm{H}), 1.36(\mathrm{~s}, 3 \mathrm{H}) .{ }^{13} \mathrm{C}$ NMR $\left(126 \mathrm{MHz}, \mathrm{CDCl}_{3}\right) \delta 152.10,136.52,136.36,135.97,135.12,134.94,132.84,132.57,130.20,129.82$, 129.80, 128.89, 128.74, 127.74, 127.72, 125.36, 22.24, 21.59, 19.81. HRMS-DART Positive calcd. for $\mathrm{C}_{23} \mathrm{H}_{25} \mathrm{OSi}[\mathrm{M}+\mathrm{H}]^{+}$345.1675, found: 345.1678 .

\subsection{General procedures for the synthesis of products 2}

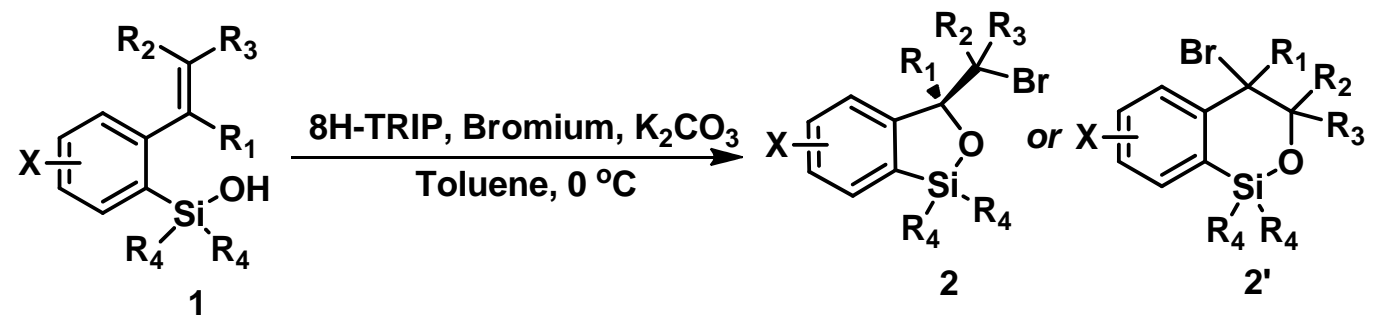


The products 2 were synthesized according to literature with little modification ${ }^{[1,2]}$.

To a mixture of 8H-R-TRIP L1 (7.6 mg, $0.01 \mathrm{mmol})$, bromium salt B1 (69.5 mg, $0.13 \mathrm{mmol})$, and $\mathrm{K}_{2} \mathrm{CO}_{3}(55.2 \mathrm{mg}, 0.4 \mathrm{mmol})$ in anhydrous toluene $(1 \mathrm{~mL})$ was added dropwise a solution of substrate 1 $(0.1 \mathrm{mmol})$ in toluene $(1 \mathrm{~mL})$ at $0{ }^{\circ} \mathrm{C}$ under argon atmosphere. The mixture was stirred at this temperature until TLC showed the complete consumption of substrate 1. The reaction mixture was filtered through a silica gel pad, washed with toluene, and concentrated in vacuum. The crude product was purified by preparative TLC to afford target product 2.

For HPLC analysis, the racemic product 2 was synthesized following the general procedure: To a mixture of substrate $1(0.1 \mathrm{mmol})$ and NBS $(0.13 \mathrm{mmol})$ under Ar was added dichloromethane $(2 \mathrm{~mL})$. The reaction was stirred at room temperature for certain hours until the starting material was consumed completely, and then the reaction was directly purified by preparative TLC to afford the racemic sample of 2 .

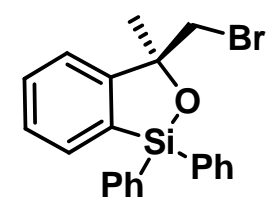

$2 a$

(S)-3-(bromomethyl)-1,3-dihydro-3-methyl-1,1-diphenylbenzo[c][1,2]oxasilole (2a): Following the general procedure, 2a was obtained from 1a (reaction time $4 \mathrm{~h}$ ) as colorless oil (34 mg, 87\%).

$\mathrm{R} f=0.5$ (petroleum ether/ethyl acetate $=30: 1$ ); $[\mathrm{a}]_{26.3}^{\mathrm{D}}=7.7\left(\right.$ c 1.40, $\left.\mathrm{CHCl}_{3}\right)$; IR (film): 3067, 2978, 2937, 1429, 1368, 1119, 1062, 957, 730, $516 \mathrm{~cm}^{-1} ;{ }^{1} \mathrm{H}$ NMR (400 MHz, $\left.\mathrm{CDCl}_{3}\right) \delta$ 7.77-7.72 (m, 3H), 7.63-7.59 (m, 2H), 7.54-7.31 (m, 9H), $3.80(\mathrm{~d}, J=10.5 \mathrm{~Hz}, 1 \mathrm{H}), 3.68(\mathrm{~d}, J=10.5 \mathrm{~Hz}, 1 \mathrm{H}), 1.79(\mathrm{~s}, 3 \mathrm{H})$. ${ }^{13} \mathrm{C}$ NMR $\left(126 \mathrm{MHz}, \mathrm{CDCl}_{3}\right) \delta 154.43,135.18,135.06,134.17,133.42,132.86,132.29,130.58,130.53$, 130.50, 128.17, 127.99, 127.98, 122.62, 84.45, 43.75, 28.59. HRMS-DART Positive calcd. for $\mathrm{C}_{21} \mathrm{H}_{20} \mathrm{BrOSi}[\mathrm{M}+\mathrm{H}]^{+}$395.0467, found: 395.0466. Enantiomeric excess was found to be $95.5 \%$ by chiral HPLC (ChiralPak OD-H column, hexane/i-PrOH 99:1 $0.3 \mathrm{~mL} / \mathrm{min}, \mathrm{t}_{\text {major }}=14.93 \mathrm{~min}, \mathrm{t}_{\text {minor }}=$ $15.75 \mathrm{~min})$.

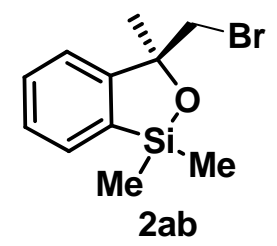

(S)-3-(bromomethyl)-1,3-dihydro-1,1,3-trimethylbenzo[c][1,2]oxasilole (2ab): Following the general procedure, 2ab was obtained from 1ab (reaction time $4 \mathrm{~h}$ ) as colorless oil (24 mg, 91\%). $\mathrm{R} f=0.5$ (petroleum ether/ethyl acetate $=35: 1) ;[\mathrm{a}]_{26.4}^{\mathrm{D}}=25.1\left(\right.$ c $\left.0.42, \mathrm{CHCl}_{3}\right) ; \mathrm{IR}$ (film): 3047, 2976, 2935, 1420, 1361, 1110, 1066, 953, 738, $526 \mathrm{~cm}^{-1} ;{ }^{1} \mathrm{H}$ NMR $\left(400 \mathrm{MHz}, \mathrm{CDCl}_{3}\right) \delta 7.55(\mathrm{~d}, J=7.5 \mathrm{~Hz}$, 1H), $7.43(\mathrm{t}, J=7.5 \mathrm{~Hz}, 1 \mathrm{H}), 7.34(\mathrm{t}, J=7.5 \mathrm{~Hz}, 1 \mathrm{H}), 7.20(\mathrm{~d}, J=7.5 \mathrm{~Hz}, 1 \mathrm{H}), 3.72(\mathrm{~d}, J=10.5 \mathrm{~Hz}$, $1 \mathrm{H}), 3.61(\mathrm{~d}, J=10.5 \mathrm{~Hz}, 1 \mathrm{H}), 1.65(\mathrm{~s}, 3 \mathrm{H}), 0.47(\mathrm{~s}, 3 \mathrm{H}), 0.38(\mathrm{~s}, 3 \mathrm{H}) .{ }^{13} \mathrm{C} \mathrm{NMR}\left(126 \mathrm{MHz}, \mathrm{CDCl}_{3}\right) \delta$ 153.47, 135.93, 130.92, 129.95, 127.70, 122.14, 83.92, 44.12, 28.89, 1.39, 0.76. HRMS-DART Positive calcd. for $\mathrm{C}_{11} \mathrm{H}_{16} \mathrm{BrOSi}[\mathrm{M}+\mathrm{H}]^{+}$271.0154, found: 271.0158. Enantiomeric excess was found to be $72 \%$ by chiral HPLC (ChiralPak OD-H column, hexane $/ \mathrm{i}-\mathrm{PrOH} 95: 50.7 \mathrm{~mL} / \mathrm{min}, \mathrm{t}_{\text {major }}=5.33 \mathrm{~min}$, $\left.\mathrm{t}_{\text {minor }}=5.96 \mathrm{~min}\right)$. 


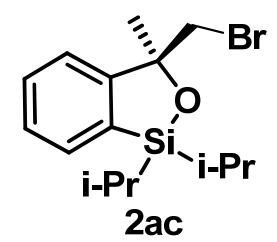

(S)-3-(bromomethyl)-1,3-dihydro-1,1-diisopropyl-3-methylbenzo[c][1,2]oxasilole (2ac): Following the general procedure, 2ac was obtained from 1ac (reaction time $4 \mathrm{~h}$ ) as colorless oil (28 $\mathrm{mg}, 89 \%)$. $\mathrm{R} f=0.5$ (petroleum ether/ethyl acetate $=35: 1) ;[\mathrm{a}]_{26.1}^{\mathrm{D}}=25.9\left(\right.$ c 0.88, $\left.\mathrm{CHCl}_{3}\right) ; \mathrm{IR}$ (film): 3069, 2974, 2933, 1426, 1363, 1109, 1068, 956, 720, $512 \mathrm{~cm}^{-1} ;{ }^{1} \mathrm{H}$ NMR $\left(400 \mathrm{MHz}, \mathrm{CDCl}_{3}\right) \delta 7.54(\mathrm{~d}, J=7.0 \mathrm{~Hz}$, 1H), $7.42(\mathrm{t}, J=7.0 \mathrm{~Hz}, 1 \mathrm{H}), 7.32(\mathrm{t}, J=7.0 \mathrm{~Hz}, 1 \mathrm{H}), 7.24(\mathrm{~d}, J=7.0 \mathrm{~Hz}, 1 \mathrm{H}), 3.68(\mathrm{~d}, J=10.5 \mathrm{~Hz}$, $1 \mathrm{H}), 3.48(\mathrm{~d}, J=10.5 \mathrm{~Hz}, 1 \mathrm{H}), 1.66(\mathrm{~s}, 3 \mathrm{H}), 1.29-1.19(\mathrm{~m}, 2 \mathrm{H}), 1.09(\mathrm{~d}, J=7.0 \mathrm{~Hz}, 3 \mathrm{H}), 1.07$ (d, $J=$ $7.0 \mathrm{~Hz}, 3 \mathrm{H}), 1.03(\mathrm{~d}, J=7.0 \mathrm{~Hz}, 3 \mathrm{H}), 0.97(\mathrm{~d}, J=7.0 \mathrm{~Hz}, 3 \mathrm{H}) .{ }^{13} \mathrm{C} \mathrm{NMR}\left(126 \mathrm{MHz}, \mathrm{CDCl}_{3}\right) \delta 154.29$, 132.60, 132.41, 129.87, 127.56, 122.58, 83.48, 44.33, 27.91, 17.47, 17.45, 17.37, 17.32, 13.15, 12.73. HRMS-DART Positive calcd. for $\mathrm{C}_{15} \mathrm{H}_{24} \mathrm{BrOSi}[\mathrm{M}+\mathrm{H}]^{+}$327.0780, found: 327.0782. Enantiomeric excess was found to be $68 \%$ by chiral HPLC (ChiralPak PC-3 column, methanol/water 8:2 0.7 $\mathrm{mL} / \mathrm{min}$, $\mathrm{t}_{\text {major }}=12.84 \mathrm{~min}, \mathrm{t}_{\text {minor }}=13.92 \mathrm{~min}$ ).<smiles>Cc1ccc2c(c1)[Si]([PH3+])([PH3+])O[C@@]2(C)CBr</smiles>

(S)-3-(bromomethyl)-1,3-dihydro-3,5-dimethyl-1,1-diphenylbenzo[c][1,2]oxasilole (2b): Following the general procedure, $\mathbf{2 b}$ was obtained from $\mathbf{1 b}$ (reaction time $4 \mathrm{~h}$ ) as colorless oil (38 $\mathrm{mg}, 94 \%)$. $\mathrm{R} f=0.5$ (petroleum ether/ethyl acetate $=30: 1) ;[\mathrm{a}]_{26.2}^{\mathrm{D}}=18.7\left(\right.$ c 1.10, $\left.\mathrm{CHCl}_{3}\right) ; \mathrm{IR}$ (film): 3047, 2972 , 2936, 1424, 1362, 1110, 1065, 937, 732, $526 \mathrm{~cm}^{-1} ;{ }^{1} \mathrm{H}$ NMR (400 MHz, $\left.\mathrm{CDCl}_{3}\right) \delta$ 7.75-7.72 (m, 2H), 7.63-7.58 (m, 3H), 7.48-7.34 (m, 6H), 7.25-7.22 (m, 1H), $7.14(\mathrm{~s}, 1 \mathrm{H}), 3.79(\mathrm{~d}, J=10.5 \mathrm{~Hz}, 1 \mathrm{H}), 3.66$ $(\mathrm{d}, J=10.5 \mathrm{~Hz}, 1 \mathrm{H}), 2.43(\mathrm{~s}, 3 \mathrm{H}), 1.77(\mathrm{~s}, 3 \mathrm{H}) .{ }^{13} \mathrm{C} \mathrm{NMR}\left(126 \mathrm{MHz}, \mathrm{CDCl}_{3}\right) \delta 154.87,140.72,135.15$, 135.04, 134.39, 133.66, 132.11, 130.48, 130.44, 129.41, 129.37, 127.94, 123.23, 84.28, 43.87, 28.58, 21.84. HRMS-DART Positive calcd. for $\mathrm{C}_{22} \mathrm{H}_{22} \mathrm{BrOSi}[\mathrm{M}+\mathrm{H}]^{+}$409.0623, found: 409.0625 . Enantiomeric excess was found to be $96 \%$ by chiral HPLC (ChiralPak PA-2 column, hexane/i-PrOH 99:1 $\left.0.4 \mathrm{~mL} / \mathrm{min}, \mathrm{t}_{\text {major }}=11.76 \mathrm{~min}, \mathrm{t}_{\text {minor }}=10.96 \mathrm{~min}\right)$.<smiles>Cc1ccc2c(c1)[Si](c1ccccc1)(c1ccccc1)O[C@@]2(C)CBr</smiles>

(S)-3-(bromomethyl)-1,3-dihydro-3,6-dimethyl-1,1-diphenylbenzo[c][1,2]oxasilole (2c): Following the general procedure, 2c was obtained from 1c (reaction time $4 \mathrm{~h}$ ) as colorless oil (37 mg, 92\%).

$\mathrm{R} f=0.5$ (petroleum ether/ethyl acetate $=30: 1) ;[\mathrm{a}]_{26.3}^{\mathrm{D}}=13.5\left(\right.$ c 0.84, $\left.\mathrm{CHCl}_{3}\right) ; \mathrm{IR}$ (film): 3041, 2973, 2938, 1424, 1360, 1120, 1069, 917, 736, $556 \mathrm{~cm}^{-1} ;{ }^{1} \mathrm{H}$ NMR (500 MHz, $\left.\mathrm{CDCl}_{3}\right) \delta$ 7.78-7.74 (m, 2H), 7.65-7.60 (m, 2H), 7.56-7.53 (m, 1H), 7.49-7.37 (m, 6H), 7.34-7.31 (m, 1H), 7.24 (d, J=8.0 Hz, 1H), $3.79(\mathrm{~d}, J=10.5 \mathrm{~Hz}, 1 \mathrm{H}), 3.67(\mathrm{~d}, J=10.5 \mathrm{~Hz}, 1 \mathrm{H}), 2.41(\mathrm{~s}, 3 \mathrm{H}), 1.79$ (s, 3H). ${ }^{13} \mathrm{C} \mathrm{NMR}(126 \mathrm{MHz}$, $\left.\mathrm{CDCl}_{3}\right) \delta 151.66,137.80,135.14,135.04,134.27,133.54,132.95,132.55,131.54,130.51,130.47$, 127.95, 122.38, 84.27, 43.90, 28.59, 21.21. HRMS-DART Positive calcd. for $\mathrm{C}_{22} \mathrm{H}_{22} \mathrm{BrOSi}[\mathrm{M}+\mathrm{H}]^{+}$ 
409.0623, found: 409.0626. Enantiomeric excess was found to be $92.5 \%$ by chiral HPLC (ChiralPak OD-H column, hexane $/ \mathrm{i}$-PrOH 99.5:0.5 0.5 mL/min, $\mathrm{t}_{\text {major }}=8.69 \mathrm{~min}, \mathrm{t}_{\text {minor }}=9.31 \mathrm{~min}$ ).<smiles>COc1ccc2c(c1)[C@](C)(CBr)O[Si]2(c1ccccc1)c1ccccc1</smiles>

2d

(S)-3-(bromomethyl)-1,3-dihydro-5-methoxy-3-methyl-1,1-diphenylbenzo[c][1,2]oxasilole (2d): Following the general procedure, 2d was obtained from 1d (reaction time $4 \mathrm{~h}$ ) as colorless oil (39 $\mathrm{mg}$, $93 \%)$.

$\mathrm{R} f=0.5$ (petroleum ether/ethyl acetate $=20: 1) ;[\mathrm{a}]_{26.2}^{\mathrm{D}}=15.7\left(\right.$ c 1.12, $\left.\mathrm{CHCl}_{3}\right) ; \mathrm{IR}$ (film): 3021, 2978, 2928, 1426, 1369, 1110, 1059, 914, 739, $550 \mathrm{~cm}^{-1} ;{ }^{1} \mathrm{H}$ NMR (400 MHz, $\left.\mathrm{CDCl}_{3}\right) \delta$ 7.78-7.68 (m, 2H), 7.67-7.55 (m, 3H), 7.54-7.29 (m, 6H), $6.97(\mathrm{dd}, J=8.0,2.0 \mathrm{~Hz}, 1 \mathrm{H}), 6.84(\mathrm{~d}, J=2.0 \mathrm{~Hz}, 1 \mathrm{H}), 3.86(\mathrm{~s}$, $3 \mathrm{H}), 3.76(\mathrm{~d}, J=10.5 \mathrm{~Hz}, 1 \mathrm{H}), 3.66(\mathrm{~d}, J=10.5 \mathrm{~Hz}, 1 \mathrm{H}), 1.77(\mathrm{~s}, 3 \mathrm{H}) .{ }^{13} \mathrm{C} \mathrm{NMR}\left(126 \mathrm{MHz}, \mathrm{CDCl}_{3}\right) \delta$ $161.92,156.71,135.14,135.03,134.51,133.77,133.43,130.47,130.42,127.94,127.93,123.65$, 114.67, 108.26, 84.12, 55.36, 43.64, 28.53. HRMS-DART Positive calcd. for $\mathrm{C}_{22} \mathrm{H}_{22} \mathrm{BrO}_{2} \mathrm{Si}[\mathrm{M}+\mathrm{H}]^{+}$ 425.0572, found: 425.0570. Enantiomeric excess was found to be $96 \%$ by chiral HPLC (ChiralPak OD-H column, hexane $/ \mathrm{i}-\mathrm{PrOH} 98: 20.7 \mathrm{~mL} / \mathrm{min}, \mathrm{t}_{\text {major }}=6.98 \mathrm{~min}, \mathrm{t}_{\text {minor }}=7.41 \mathrm{~min}$ ).<smiles>C[C@]1(CBr)O[Si](c2ccccc2)(c2ccccc2)c2ccc(Cl)cc21</smiles>

(S)-3-(bromomethyl)-5-chloro-1,3-dihydro-3-methyl-1,1-diphenylbenzo[c][1,2]oxasilole

Following the general procedure, 2e was obtained from 1e (reaction time $4 \mathrm{~h}$ ) as colorless oil (38.5 mg, $92 \%)$.

$\mathrm{R} f=0.5$ (petroleum ether/ethyl acetate $=30: 1) ;[\mathrm{a}]_{26.3}^{\mathrm{D}}=19.2\left(c 0.84, \mathrm{CHCl}_{3}\right) ; \mathrm{IR}$ (film): 3010, 2978, 2930, 1436, 1365, 1113, 1056, 912, 739, $560 \mathrm{~cm}^{-1} ;{ }^{1} \mathrm{H}$ NMR (400 MHz, $\left.\mathrm{CDCl}_{3}\right) \delta$ 7.72-7.68 (m, 2H), $7.63(\mathrm{~d}, J=7.5 \mathrm{~Hz}, 1 \mathrm{H}), 7.60-7.54(\mathrm{~m}, 2 \mathrm{H}), 7.49-7.32(\mathrm{~m}, 8 \mathrm{H}), 3.75(\mathrm{~d}, J=10.5 \mathrm{~Hz}, 1 \mathrm{H}), 3.65(\mathrm{~d}, J=$ $10.5 \mathrm{~Hz}, 1 \mathrm{H}), 1.77$ (s, 3H). ${ }^{13} \mathrm{C}$ NMR $\left(126 \mathrm{MHz}, \mathrm{CDCl}_{3}\right) \delta 156.48,137.03,135.17,134.98,133.64$, 133.40, 132.80, 131.31, 130.77, 130.74, 128.60, 128.07, 128.03, 123.03, 84.16, 43.05, 28.54. HRMS-DART Positive calcd. for $\mathrm{C}_{21} \mathrm{H}_{19} \mathrm{BrClOSi}[\mathrm{M}+\mathrm{H}]^{+}$429.0077, found: 429.0074. Enantiomeric excess was found to be $98 \%$ by chiral HPLC (ChiralPak OD-H column, hexane/i-PrOH 100:0 0.5 $\mathrm{mL} / \mathrm{min}, \mathrm{t}_{\text {major }}=12.55 \mathrm{~min}, \mathrm{t}_{\text {minor }}=13.72 \mathrm{~min}$ ).<smiles>[Z17][Si](O[C@@]1(C)O[C@@](C)(CBr)c2ccc(Cl)cc21)(c1ccccc1)c1ccc(Cl)cc1</smiles>

(S)-3-(bromomethyl)-6-chloro-1,3-dihydro-3-methyl-1,1-diphenylbenzo[c][1,2]oxasilole

Following the general procedure, $2 \mathbf{f}$ was obtained from 1 f (reaction time $4 \mathrm{~h}$ ) as colorless oil (38 $\mathrm{mg}$, $91 \%)$

$\mathrm{R} f=0.5$ (petroleum ether/ethyl acetate $=40: 1) ;[\mathrm{a}]_{26.5}^{\mathrm{D}}=10.4\left(\right.$ c 0.64, $\left.\mathrm{CHCl}_{3}\right) ; \mathrm{IR}$ (film): 3013, 2976, 2936, 1426, 1365, 1118, 1050, 913, 735, $561 \mathrm{~cm}^{-1} ;{ }^{1} \mathrm{H}$ NMR (400 MHz, $\left.\mathrm{CDCl}_{3}\right) \delta$ 7.74-7.68 (m, 2H), 
$7.64(\mathrm{~d}, J=2.0 \mathrm{~Hz}, 1 \mathrm{H}), 7.61-7.55(\mathrm{~m}, 2 \mathrm{H}), 7.49-7.36(\mathrm{~m}, 7 \mathrm{H}), 7.25(\mathrm{~d}, J=3.0 \mathrm{~Hz}, 1 \mathrm{H}), 3.74(\mathrm{~d}, J=$ $10.5 \mathrm{~Hz}, 1 \mathrm{H}), 3.65(\mathrm{~d}, J=10.5 \mathrm{~Hz}, 1 \mathrm{H}), 1.76(\mathrm{~s}, 3 \mathrm{H}) .{ }^{13} \mathrm{C} \mathrm{NMR}\left(126 \mathrm{MHz}, \mathrm{CDCl}_{3}\right) \delta 152.67,135.64$, 135.18, 134.98, 134.48, 133.40, 132.58, 131.79, 130.84, 130.77, 130.66, 128.09, 128.07, 124.03, 84.23, 43.19, 28.57. HRMS-DART Positive calcd. for $\mathrm{C}_{21} \mathrm{H}_{19} \mathrm{BrClOSi}[\mathrm{M}+\mathrm{H}]^{+} 429.0077$, found: 429.0075 . Enantiomeric excess was found to be $94 \%$ by chiral HPLC (ChiralPak OD-H column, hexane/i-PrOH 100:0 $0.7 \mathrm{~mL} / \mathrm{min}, \mathrm{t}_{\text {major }}=30.03 \mathrm{~min}, \mathrm{t}_{\text {minor }}=33.92 \mathrm{~min}$ ).<smiles>[Z9][PH]1(c2ccc(C(F)(F)F)cc2)O[C@](C)(CBr)c2ccccc21</smiles>

(S)-3-(bromomethyl)-5-(trifluoromethyl)-1,3-dihydro-3-methyl-1,1-diphenylbenzo[c][1,2]oxasilole (2g): Following the general procedure, $\mathbf{2 g}$ was obtained from $\mathbf{1 g}$ (reaction time $4 \mathrm{~h}$ ) as colorless oil (41 $\mathrm{mg}, 90 \%)$.

$\mathrm{R} f=0.5$ (petroleum ether/ethyl acetate $=40: 1) ;[\mathrm{a}]^{\mathrm{D}} 26.4=3.4\left(\right.$ c $\left.0.88, \mathrm{CHCl}_{3}\right) ; \mathrm{IR}($ film): 3007, 2974, 2935, 1420, 1355, 1108, 1053, 903, 725, $560 \mathrm{~cm}^{-1} ;{ }^{19} \mathrm{~F}$ NMR $\left(376 \mathrm{MHz}, \mathrm{CDCl}_{3}\right) \delta-62.60$ (s). ${ }^{1} \mathrm{H}$ NMR $\left(400 \mathrm{MHz}, \mathrm{CDCl}_{3}\right) \delta 7.84(\mathrm{~d}, J=7.5 \mathrm{~Hz}, 1 \mathrm{H}), 7.77-7.68(\mathrm{~m}, 2 \mathrm{H}), 7.65(\mathrm{~d}, J=7.5 \mathrm{~Hz}, 1 \mathrm{H}), 7.60-7.55$ $(\mathrm{m}, 3 \mathrm{H}), 7.52-7.35(\mathrm{~m}, 6 \mathrm{H}), 3.78(\mathrm{~d}, J=10.5 \mathrm{~Hz}, 1 \mathrm{H}), 3.69(\mathrm{~d}, J=10.5 \mathrm{~Hz}, 1 \mathrm{H}), 1.81(\mathrm{~s}, 3 \mathrm{H}) .{ }^{13} \mathrm{C}$ NMR $\left(126 \mathrm{MHz}, \mathrm{CDCl}_{3}\right) \delta 155.20,137.87,135.19,134.97,133.19,132.85,132.35,130.93,130.86$, 128.14, 128.10, 124.95 (q, $J=3.9 \mathrm{~Hz}$ ), 119.29 (q, $J=3.9 \mathrm{~Hz}$ ), 84.49, 42.89, 28.58. HRMS-DART Positive calcd. for $\mathrm{C}_{22} \mathrm{H}_{19} \mathrm{BrF}_{3} \mathrm{OSi}[\mathrm{M}+\mathrm{H}]^{+} 463.0341$, found: 463.0340 . Enantiomeric excess was found to be $99 \%$ by chiral HPLC (ChiralPak OD-H column, hexane $/ \mathrm{i}-\mathrm{PrOH}$ 100:0 $0.5 \mathrm{~mL} / \mathrm{min}$, $\mathrm{t}_{\text {major }}=10.61$ $\min , \mathrm{t}_{\text {minor }}=11.57 \mathrm{~min}$ ).<smiles>Cc1cccc2c1[Si](c1ccccc1)(c1ccccc1)OC2(C)CBr</smiles>

$2 h$

(S)-3-(bromomethyl)-1,3-dihydro-3,7-dimethyl-1,1-diphenylbenzo[c][1,2]oxasilole (2h): Following the general procedure, $2 \mathbf{h}$ was obtained from $\mathbf{1 h}$ (reaction time $6 \mathrm{~h}$ ) as colorless oil (31 $\mathrm{mg}, 75 \%$ ).

$\mathrm{R} f=0.5$ (petroleum ether/ethyl acetate $=30: 1) ;[\mathrm{a}]_{26.2}^{\mathrm{D}}=3.1\left(\right.$ c 1.51, $\left.\mathrm{CHCl}_{3}\right) ; \mathrm{IR}($ film): 3012, 2972, 2937, 1426, 1345, 1118, 1043, 913, 728, $562 \mathrm{~cm}^{-1} ;{ }^{1} \mathrm{H}$ NMR (500 MHz, CDCl 3$) \delta$ 7.80-7.77 (m, 2H), 7.67-7.64 (m, 2H), 7.51-7.39 (m, 7H), 7.22-7.16 (m, 2H), $3.82(\mathrm{~d}, J=10.5 \mathrm{~Hz}, 1 \mathrm{H}), 3.70(\mathrm{~d}, J=10.5$ $\mathrm{Hz}, 1 \mathrm{H}), 2.41$ (s, 3H), 1.80 (s, 3H). ${ }^{13} \mathrm{C}$ NMR $\left(126 \mathrm{MHz}, \mathrm{CDCl}_{3}\right) \delta 154.67,142.78,135.63,135.35$, 133.75, 133.01, 132.55, 131.08, 130.61, 130.54, 128.69, 128.01, 127.99, 119.89, 84.30, 43.88, 28.71, 23.32. HRMS-DART Positive calcd. for $\mathrm{C}_{22} \mathrm{H}_{22} \mathrm{BrOSi}[\mathrm{M}+\mathrm{H}]^{+}$409.0623, found: 409.0620 . Enantiomeric excess was found to be $76 \%$ by chiral HPLC (ChiralPak OD-H column, hexane/i-PrOH 100:0 $0.5 \mathrm{~mL} / \mathrm{min}, \mathrm{t}_{\text {major }}=12.77 \mathrm{~min}, \mathrm{t}_{\text {minor }}=14.11 \mathrm{~min}$ ).<smiles>C[C@]1(CBr)O[Si](c2ccccc2)(c2ccccc2)c2c1ccc1ccccc21</smiles>

2i

(S)-3-(bromomethyl)-1,3-dihydro-3-methyl-1,1-diphenylnaphtho[1,2-c][1,2]oxasilole

(2i): 
Following the general procedure, $2 \mathbf{i}$ was obtained from $1 \mathbf{i}$ (reaction time $6 \mathrm{~h}$ ) as colorless oil (35 $\mathrm{mg}$, $81 \%)$.

$\mathrm{R} f=0.5$ (petroleum ether/ ethyl acetate $=25: 1) ;[\mathrm{a}]_{26.2}^{\mathrm{D}}=-6.0\left(c 1.32, \mathrm{CHCl}_{3}\right) ; \mathrm{IR}($ film): 3010, 2978, 2930, 1423, 1325, 1116, 1033, 923, 725, $542 \mathrm{~cm}^{-1} ;{ }^{1} \mathrm{H}$ NMR (500 MHz, $\left.\mathrm{CDCl}_{3}\right) \delta 8.02(\mathrm{~d}, J=8.5 \mathrm{~Hz}$, $1 \mathrm{H}), 7.95-7.93(\mathrm{~m}, 1 \mathrm{H}), 7.88-7.83(\mathrm{~m}, 3 \mathrm{H}), 7.75-7.70(\mathrm{~m}, 2 \mathrm{H}), 7.54-7.38(\mathrm{~m}, 9 \mathrm{H}), 3.93(\mathrm{~d}, J=10.5 \mathrm{~Hz}$, $1 \mathrm{H}), 3.80(\mathrm{~d}, J=10.5 \mathrm{~Hz}, 1 \mathrm{H}), 1.88(\mathrm{~s}, 3 \mathrm{H}) .{ }^{13} \mathrm{C} \mathrm{NMR}\left(126 \mathrm{MHz}, \mathrm{CDCl}_{3}\right) \delta 154.18,135.70,135.33$, $134.00,133.25,133.05,131.80,131.13,130.69,130.59,129.11,128.65,128.06,128.02,127.25$, 126.24, 120.62, 84.81, 43.38, 28.49. HRMS-DART Positive calcd. for $\mathrm{C}_{25} \mathrm{H}_{22} \mathrm{BrOSi}[\mathrm{M}+\mathrm{H}]^{+} 445.0623$, found: 445.0625 . Enantiomeric excess was found to be $84 \%$ by chiral HPLC (ChiralPak OD-H column, hexane/i-PrOH 99:1 $0.7 \mathrm{~mL} / \mathrm{min}, \mathrm{t}_{\text {major }}=8.42 \mathrm{~min}, \mathrm{t}_{\text {minor }}=9.93 \mathrm{~min}$ ).<smiles>CC[C@@]1(CBr)O[Si]([TlH])(c2ccccc2)c2ccccc21</smiles>

(S)-3-(bromomethyl)-3-ethyl-1,3-dihydro-1,1-diphenylbenzo[c][1,2]oxasilole (2j): Following the general procedure, $\mathbf{2 j}$ was obtained from $\mathbf{1} \mathbf{j}$ (reaction time $6 \mathrm{~h}$ ) as colorless oil (37 $\mathrm{mg}, 91 \%$ ).

$\mathrm{R} f=0.5$ (petroleum ether/ethyl acetate $=40: 1) ;[\mathrm{a}]_{26.3}^{\mathrm{D}}=23.4\left(c 1.24, \mathrm{CHCl}_{3}\right) ; \mathrm{IR}($ film): 3005, 2972, 2933, 1420, 1315, 1114, 1023, 927, 735, $547 \mathrm{~cm}^{-1} ;{ }^{1} \mathrm{H}$ NMR (500 MHz, $\left.\mathrm{CDCl}_{3}\right) \delta$ 7.81-7.73 (m, 3H), 7.65-7.60 (m, 2H), $7.52(\mathrm{t}, J=7.5 \mathrm{~Hz}, 1 \mathrm{H}), 7.47-7.34(\mathrm{~m}, 8 \mathrm{H}), 3.77(\mathrm{~d}, J=10.5 \mathrm{~Hz}, 1 \mathrm{H}), 3.69(\mathrm{~d}, J=$ $10.5 \mathrm{~Hz}, 1 \mathrm{H}), 2.29$ (dq, $J=14.5,7.0 \mathrm{~Hz}, 1 \mathrm{H}), 2.00(\mathrm{dq}, J=14.5,7.0 \mathrm{~Hz}, 1 \mathrm{H}), 0.79$ (t, $J=7.0 \mathrm{~Hz}, 3 \mathrm{H})$. ${ }^{13} \mathrm{C}$ NMR $\left(126 \mathrm{MHz}, \mathrm{CDCl}_{3}\right) \delta 153.12,135.23,135.02,133.99,133.61,132.36,130.51,130.44,130.41$, $128.11,127.94,127.89,122.99,87.28,43.05,32.74,8.46$. HRMS-DART Positive calcd. for $\mathrm{C}_{22} \mathrm{H}_{22} \mathrm{BrOSi}[\mathrm{M}+\mathrm{H}]^{+}$409.0623, found: 409.0622. Enantiomeric excess was found to be $87.5 \%$ by chiral HPLC (ChiralPak PC-3 column, methanol/water 9:1 $0.7 \mathrm{~mL} / \mathrm{min}, \mathrm{t}_{\text {major }}=12.67 \mathrm{~min}, \mathrm{t}_{\text {minor }}=14.19$ $\min )$.<smiles>CC(C)[C@]1(CBr)O[Si](c2ccccc2)(c2ccccc2)c2ccccc21</smiles>

(S)-3-(bromomethyl)-1,3-dihydro-3-isopropyl-1,1-diphenylbenzo[c][1,2]oxasilole (2k): Following the general procedure, $\mathbf{2 k}$ was obtained from $\mathbf{1 k}$ (reaction time $10 \mathrm{~h}$ ) as colorless oil (37 $\mathrm{mg}, 89 \%$ ).

$\mathrm{R} f=0.5$ (petroleum ether/ethyl acetate $=40: 1) ;[\mathrm{a}]^{\mathrm{D}} 26.2=4.1\left(\right.$ c 1.04, $\left.\mathrm{CHCl}_{3}\right)$; IR (film): 3009, 2974, 2936, 1422, 1319, 1104, 1013, 924, 725, $540 \mathrm{~cm}^{-1} ;{ }^{1} \mathrm{H}$ NMR $\left(500 \mathrm{MHz}, \mathrm{CDCl}_{3}\right) \delta 7.80(\mathrm{~d}, J=7.0 \mathrm{~Hz}$, $1 \mathrm{H})$, 7.73-7.63 (m, 4H), 7.55-7.50 (m, 2H), 7.48-7.36 (m, 7H), $3.87(\mathrm{~d}, J=10.5 \mathrm{~Hz}, 1 \mathrm{H}), 3.71(\mathrm{~d}, J=$ $10.5 \mathrm{~Hz}, 1 \mathrm{H}), 2.53(\mathrm{dq}, J=13.5,7.0 \mathrm{~Hz}, 1 \mathrm{H}), 1.10(\mathrm{~d}, J=7.0 \mathrm{~Hz}, 3 \mathrm{H}), 0.69(\mathrm{~d}, J=7.0 \mathrm{~Hz}, 3 \mathrm{H}) .{ }^{13} \mathrm{C}$ NMR $\left(126 \mathrm{MHz}, \mathrm{CDCl}_{3}\right) \delta 153.77,135.15,135.11,134.48,134.17,133.27,132.25,130.37,130.13$, 127.93, 127.89, 127.86, 123.62, 89.23, 41.25, 35.29, 18.13, 16.68. HRMS-DART Positive calcd. for $\mathrm{C}_{23} \mathrm{H}_{24} \mathrm{BrOSi}[\mathrm{M}+\mathrm{H}]^{+} 423.0780$, found: 423.0782 . Enantiomeric excess was found to be $59 \%$ by chiral HPLC (ChiralPak PC-2 column, acetonitrile/water $55: 450.7 \mathrm{~mL} / \mathrm{min}, \mathrm{t}_{\text {major }}=62.16 \mathrm{~min}, \mathrm{t}_{\text {minor }}=65.24$ $\min )$. 
<smiles>CC1(C)O[Si](c2ccccc2)(c2ccccc2)c2ccccc2[C@H]1Br</smiles>

(S)-4-bromo-3,4-dihydro-3,3-dimethyl-1,1-diphenyl-1H-benzo[c][1,2]oxasiline (2'l): Following the general procedure, 2'l was obtained from 1'l (reaction time $8 \mathrm{~h}$ ) as colorless oil (35 $\mathrm{mg}, 87 \%$ ). $\mathrm{R} f=0.5$ (petroleum ether/ethyl acetate $=35: 1) ;[\mathrm{a}]_{26.2}^{\mathrm{D}}=75.2\left(c 0.68, \mathrm{CHCl}_{3}\right) ; \mathrm{IR}$ (film): 3001, 2973, 2937, 1428, 1310, 1108, 1023, 928, 723, $530 \mathrm{~cm}^{-1} ;{ }^{1} \mathrm{H}$ NMR (500 MHz, $\left.\mathrm{CDCl}_{3}\right) \delta$ 7.77-7.73 (m, 2H), 7.63-7.57 (m, 3H), 7.45-7.30 (m, 9H), 5.15 (s, 1H), 1.69 (s, 3H), 1.43 (s, 3H). ${ }^{13} \mathrm{C}$ NMR (126 MHz, $\left.\mathrm{CDCl}_{3}\right) \delta 146.82,135.41,135.39,135.30,135.08,134.33,130.57,130.11,130.03,130.01,129.02$, 127.94, 127.85, 127.62, 75.93, 62.97, 32.01, 28.24. HRMS-DART Positive calcd. for $\mathrm{C}_{22} \mathrm{H}_{22} \mathrm{BrOSi}$ $[\mathrm{M}+\mathrm{H}]^{+} 409.0623$, found: 409.0625 . Enantiomeric excess was found to be $83 \%$ by chiral HPLC (ChiralPak PC-3 column, acetonitrile/water 7:3 $0.7 \mathrm{~mL} / \mathrm{min}, \mathrm{t}_{\text {major }}=9.67 \mathrm{~min}, \mathrm{t}_{\text {minor }}=9.01 \mathrm{~min}$ ).

Note: The exact structure of 2'I was confirmed by reduction of bromine, the reductive product has no optical rotation. Additionally its $g H S Q C$ spectrum displayed correlation of $C-4$ with two protons, which showed that product resulted from the 6-endo cyclization.

\section{Reduction of 2'l was conducted as follow.}

To a solution of 2'l (41 mg, $0.1 \mathrm{mmol})$ and $(n-\mathrm{Bu})_{3} \mathrm{SnH}(43.6 \mathrm{mg}, 0.15 \mathrm{mmol})$ in toluene $(1 \mathrm{~mL})$ under Ar at $70{ }^{\circ} \mathrm{C}$ was added AIBN $(1.6 \mathrm{mg}, 0.01 \mathrm{mmol})$ in one portion and the mixture was stirred at the same temperature for $5 \mathrm{~h}$. The reaction was cooled to room temperature and condensed. The residue was purified by preparative TLC (petroleum ether/ethyl acetate $=50: 1$ ) to afford the target product as colorless oil (31 mg, 94\%).

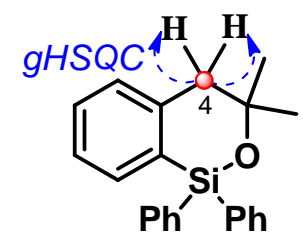

2'।

3,4-dihydro-3,3-dimethyl-1,1-diphenyl-1H-benzo[c][1,2]oxasiline (3'l): $\mathrm{R} f=0.5$ (petroleum ether/ethyl acetate $=50: 1$ ); IR (film): 3001, 2976, 2934, 1420, 1312, 1104, 1026, 918, 724, $515 \mathrm{~cm}^{-1}$; ${ }^{1} \mathrm{H}$ NMR $\left(400 \mathrm{MHz}, \mathrm{CDCl}_{3}\right)$ 87.65-7.58 (m, 4H), 7.50-7.34 (m, 8H), 7.28-7.22 (m, 1H), 7.17-7.14 (m, $1 \mathrm{H}), 2.94(\mathrm{~s}, 2 \mathrm{H}), 1.40(\mathrm{~s}, 6 \mathrm{H}) .{ }^{13} \mathrm{C} \mathrm{NMR}\left(126 \mathrm{MHz}, \mathrm{CDCl}_{3}\right) \delta 145.05,135.19,135.06,134.02,132.07$, 130.09, 129.97, 128.88, 127.81, 125.92, 74.66, 46.96, 30.37. HRMS-DART Positive calcd. for $\mathrm{C}_{22} \mathrm{H}_{23} \mathrm{OSi}[\mathrm{M}+\mathrm{H}]^{+} 331.1518$, found: 331.1812 .

Note: The absolute configuration of 2'l was established by VCD and IR studies.

\section{VCD and IR experimental of 2'l.}

BioTools ChiralIR-2X FT-VCD spectrometer, equipped with a single photoelastic modulation (PEM) and a mercury cadmium tellurium (MCT) detector, was used to record the VCD and IR spectra. A solution of 2'l $(40 \mathrm{mg})$ in $\mathrm{CDCl}_{3}(150 \mu \mathrm{L})$ was placed in a $\mathrm{BaF}_{2}$ cell with a path length of $75 \mu \mathrm{m}$. Data were acquired at a resolution of $4 \mathrm{~cm}^{-1}$ for $3 \mathrm{~h}$. The racemic sample was measured under the same 
conditions to obtain VCD baseline.

\section{VCD and IR calculations}

Conformational analysis of (S)-2'l was performed with Compute VOA (BioTools Inc., Jupiter, FL) using the Monte Carlo protocol at the molecular mechanic force field MMFF94 level. Within a 20 $\mathrm{kcal} / \mathrm{mol}$ window, four energetically distinct conformers were predicted. Geometry optimization and frequencies calculation were carried out using the B3PW91 hybrid density functional and LANL2DZ basis set with Gaussian 09 (Gaussian Inc., Wallingford, CT). Boltzmann-population-weighted composite VCD and IR spectra were then generated by Compute VOA.

Comparisons of experimental and calculated VCD and IR spectra can be seen in Fig. S1. A scaling factor of 0.96, obtained from Compare VOA (BioTools Inc., Jupiter, FL), has been applied to the calculated VCD and IR frequencies. The comparisons establish the absolute configuration of $\mathbf{2 c}$ as $(S)$.

The assignment was evaluated by Compare VOA. Table S6 shows the related result, including spectral similarities and enantiomeric similarity index (the difference between the VCD spectral similarity of the correct and the incorrect enantiomers, ESI). The confidence level of the (S) assignment is $99 \%$, based on the current Compare VOA database consisting of 105 previous correct assignments for different chiral structures.
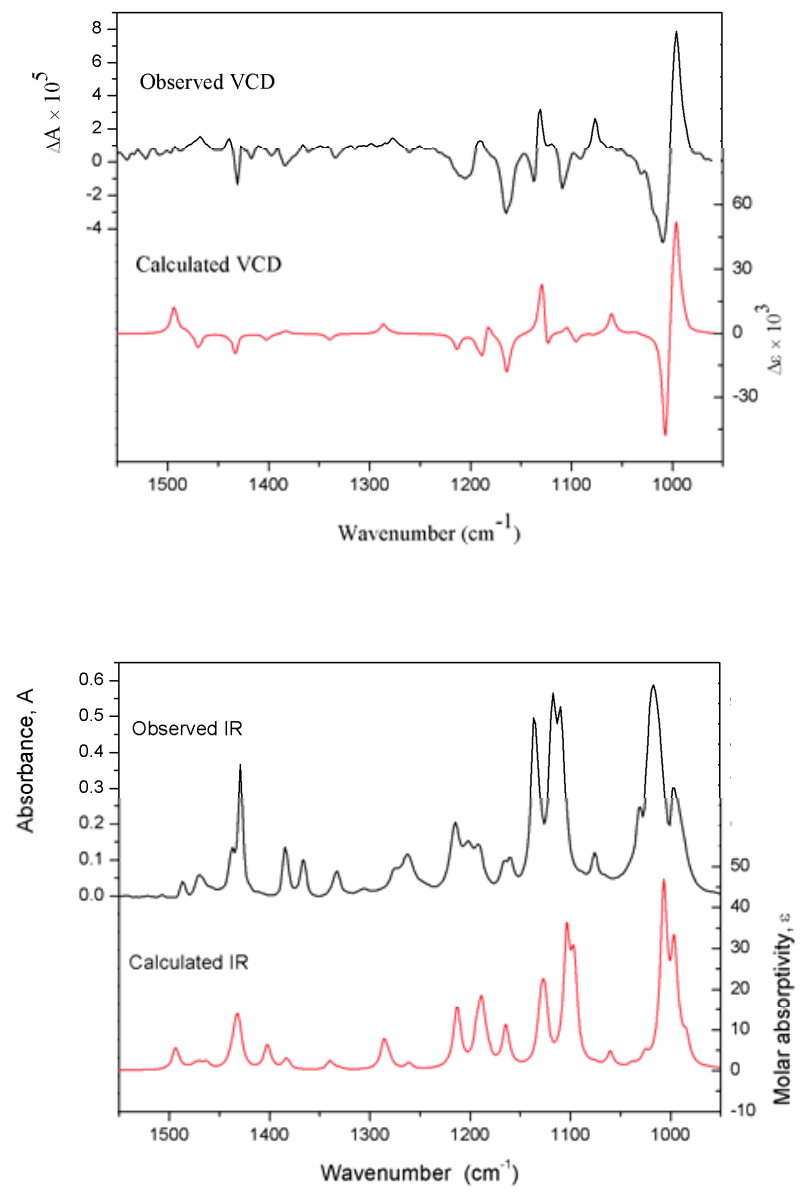

Figure S1. VCD and IR spectra observed for 2'l compared with the corresponding calculated spectra 
Table S6. Compare VOA result for VCD and IR spectra of 2'l

\begin{tabular}{ccccc}
\hline Calculation Method & ${ }^{\mathrm{a}} S_{I R}$ & ${ }^{\mathrm{b}} S_{R}$ & ${ }^{\mathrm{c}} S_{S}$ & ${ }^{\mathrm{d}} E S I$ \\
\hline DFT//B3PW91/LANL2DZ & 73.9 & 7.5 & 77.5 & 70.0 \\
\hline
\end{tabular}

${ }^{\mathrm{a}}$ IR spectral similarity

${ }^{\mathrm{b}} \mathrm{VCD}$ spectral similarity for the $(R)$-configuration

${ }^{\mathrm{c}} \mathrm{VCD}$ spectral similarity for the $(S)$-configuration

${ }^{\mathrm{d}}$ Enantiomeric similarity index.

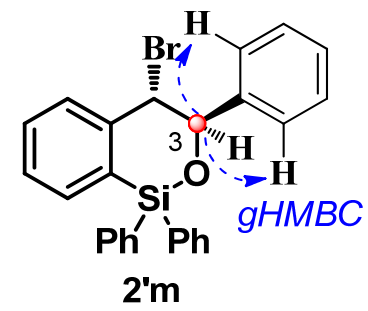

(3R,4S)-4-bromo-3,4-dihydro-1,1,3-triphenyl-1H-benzo[c][1,2]oxasiline (2'm): Following the general procedure, 2'm was obtained from 1'm (reaction time $24 \mathrm{~h}$ ) as colorless oil (23 mg, 52\%).

Note: The exact structure of 2'm was established by 2D-NMR studies with key correlation of C-3 with two proton of the appendage phenl in $g H M B C$ spectrum.

$\mathrm{R} f=0.5$ (petroleum ether/ethyl acetate $=25: 1) ;[\mathrm{a}]_{26.1}^{\mathrm{D}}=33.2\left(\right.$ c $\left.0.18, \mathrm{CHCl}_{3}\right) ; \mathrm{IR}$ (film): 3001, 2973, 2937, 1428, 1310, 1108, 1023, 928, 723, $530 \mathrm{~cm}^{-1} ;{ }^{1} \mathrm{H}$ NMR (500 MHz, $\left.\mathrm{CDCl}_{3}\right) \delta$ 7.92-7.88 (m, 2H), 7.71-7.62 (m, 3H), $7.60(\mathrm{~d}, J=7.5 \mathrm{~Hz}, 2 \mathrm{H}), 7.58-7.29(\mathrm{~m}, 12 \mathrm{H}), 5.51(\mathrm{~s}, 1 \mathrm{H}), 5.41(\mathrm{~s}, 1 \mathrm{H}) .{ }^{13} \mathrm{C}$ NMR $\left(126 \mathrm{MHz}, \mathrm{CDCl}_{3}\right) \delta 147.65,141.34,135.61,135.26,134.95,133.79,133.006,130.61,130.54,130.57$, 129.80, 129.11, 128.39, 128.06. 127.93, 127.71, 126.01, 75.40, 59.34. HRMS-DART Positive calcd. for $\mathrm{C}_{26} \mathrm{H}_{22} \mathrm{BrOSi}[\mathrm{M}+\mathrm{H}]^{+} 457.0623$, found: 457.0620 . Enantiomeric excess was found to be $65 \%$ by chiral HPLC (ChiralPak PC-1 column, acetonitrile/water $8: 20.7 \mathrm{~mL} / \mathrm{min}, \mathrm{t}_{\text {major }}=11.98 \mathrm{~min}$, $\mathrm{t}_{\text {minor }}=13.06$ $\min )$.<smiles>C[C@H](Br)[C@]1(C)O[Si](c2ccccc2)(c2ccccc2)c2ccccc21</smiles>

(S)-3-((R)-1-bromoethyl)-1,3-dihydro-3-methyl-1,1-diphenylbenzo[c][1,2]oxasilole (2n): Following the general procedure, $2 \mathbf{n}$ was obtained from $1 \mathrm{n}$ (reaction time $6 \mathrm{~h}$ ) as colorless oil (36 mg, 89\%).

$\mathrm{R} f=0.5$ (petroleum ether/ethyl acetate $=35: 1) ;[\mathrm{a}]_{22.4}^{\mathrm{D}}=-8.3\left(\right.$ c 3.95, $\left.\mathrm{CHCl}_{3}\right)$; IR (film): 3009, 2977 , 2936, 1423, 1319, 1128, 1027, 918, 729, $520 \mathrm{~cm}^{-1} ;{ }^{1} \mathrm{H}$ NMR (400 MHz, $\left.\mathrm{CDCl}_{3}\right) \delta$ 7.76-7.65 (m, 3H), 7.59-7.54 (m, 2H), 7.50-7.30 (m, 9H), 4.47 (q, $J=7.0 \mathrm{~Hz}, 1 \mathrm{H}), 1.84(\mathrm{~s}, 3 \mathrm{H}), 1.34$ (d, $J=7.0 \mathrm{~Hz}, 3 \mathrm{H})$. ${ }^{13} \mathrm{C}$ NMR $\left(126 \mathrm{MHz}, \mathrm{CDCl}_{3}\right) \delta 154.37,135.13,135.05,134.37,133.33,132.87,132.48,130.53,130.44$, 127.99, 127.98, 127.89, 122.74, 87.58, 58.84, 30.47, 21.65. HRMS-DART Positive calcd. for $\mathrm{C}_{22} \mathrm{H}_{22} \mathrm{BrOSi}[\mathrm{M}+\mathrm{H}]^{+}$409.0623, found: 409.0625. Enantiomeric excess was found to be $93.5 \%$ by chiral HPLC (ChiralPak AD-H column, hexane $/ \mathrm{i}-\mathrm{PrOH} 99: 10.7 \mathrm{~mL} / \mathrm{min}, \mathrm{t}_{\text {major }}=6.99 \mathrm{~min}, \mathrm{t}_{\text {minor }}=9.02$ $\min )$. 
<smiles>CCCC(Br)[C@]1(C)O[Si]([OH2+])(c2ccccc2)c2ccccc21</smiles>

(S)-3-((R)-1-bromobutyl)-1,3-dihydro-3-methyl-1,1-diphenylbenzo[c][1,2]oxasilole (2o): Following the general procedure, $2 \mathbf{o}$ was obtained from 10 (reaction time $6 \mathrm{~h}$ ) as colorless oil (39 $\mathrm{mg}, 90 \%$ ). $\mathrm{R} f=0.5$ (petroleum ether/ethyl acetate $=40: 1) ;[\mathrm{a}]_{23.3}^{\mathrm{D}}=-29.2\left(\mathrm{c} 1.83, \mathrm{CHCl}_{3}\right) ; \mathrm{IR}$ (film): 3004, 2975, 2936, 1420 1310, 1123, 1017, 912, 720, $523 \mathrm{~cm}^{-1}$; ${ }^{1} \mathrm{H}$ NMR (400 MHz, $\left.\mathrm{CDCl}_{3}\right) \delta$ 7.74-7.71 (m, 1H), 7.69-7.65 (m, 2H), 7.61-7.56 (m, 2H), 7.51-7.31 (m, 9H), 4.28-4.23 (m, 1H), $1.85(\mathrm{~s}, 3 \mathrm{H}), 1.63-1.47(\mathrm{~m}$, $2 \mathrm{H}), 1.36-1.26(\mathrm{~m}, 1 \mathrm{H}), 1.22-1.13(\mathrm{~m}, 1 \mathrm{H}), 0.63(\mathrm{t}, J=7.0 \mathrm{~Hz}, 3 \mathrm{H}) .{ }^{13} \mathrm{C}$ NMR $\left(126 \mathrm{MHz}, \mathrm{CDCl}_{3}\right) \delta$ $155.05,135.21,135.09,134.26,133.40,132.92,132.47,130.53,130.47,130.45,127.92,127.90$, 122.91, 87.80, 66.06, 35.89, 30.42, 21.17, 12.95. HRMS-DART Positive calcd. for $\mathrm{C}_{24} \mathrm{H}_{26} \mathrm{BrOSi}$ $[\mathrm{M}+\mathrm{H}]^{+}$437.0936, found: 437.0938. Enantiomeric excess was found to be $90 \%$ by chiral HPLC (ChiralPak AD-H column, hexane $/ \mathrm{i}-\mathrm{PrOH} 100: 00.7 \mathrm{~mL} / \mathrm{min}, \mathrm{t}_{\text {major }}=8.51 \mathrm{~min}, \mathrm{t}_{\text {minor }}=7.09 \mathrm{~min}$ ).<smiles>C[C@]1(C(Br)c2ccccc2)O[Si](c2ccccc2)(c2ccccc2)c2ccccc21</smiles>

(S)-3-((R)-bromo(phenyl)methyl)-1,3-dihydro-3-methyl-1,1-diphenylbenzo[c][1,2]oxasilole (2p): Following the general procedure, $\mathbf{2 p}$ was obtained from $\mathbf{1 p}$ (reaction time $24 \mathrm{~h}$ ) as colorless oil (41 mg, $89 \%)$.

$\mathrm{R} f=0.5$ (petroleum ether/ethyl acetate $=25: 1) ;[\mathrm{a}]_{22.7}^{\mathrm{D}}=-80.2\left(c 0.55, \mathrm{CHCl}_{3}\right) ; \mathrm{IR}(\mathrm{film}): 3009,2972$, 2934, 1427 1320, 1128, 1037, 917, 724, $526 \mathrm{~cm}^{-1}$; ${ }^{1} \mathrm{H}$ NMR (500 MHz, $\left.\mathrm{CDCl}_{3}\right) \delta$ 7.61-7.53 (m, 3H), 7.49-7.30 (m, 11H), 7.05-7.02 (m, 2H), 6.95-6.90 (m, 1H), 6.75-6.70 (m, 2H), $5.38(\mathrm{~s}, 1 \mathrm{H}), 1.98(\mathrm{~s}, 3 \mathrm{H})$. ${ }^{13} \mathrm{C}$ NMR $\left(126 \mathrm{MHz}, \mathrm{CDCl}_{3}\right) \delta 153.99,137.66,135.50,134.85,134.71,133.03,132.54,132.41,130.37$, 130.22, 130.07, 129.96, 127.81, 127.70, 127.49, 127.12, 123.45, 88.13, 63.84, 31.38. HRMS-DART Positive calcd. for $\mathrm{C}_{27} \mathrm{H}_{24} \mathrm{BrOSi}[\mathrm{M}+\mathrm{H}]^{+} 471.0780$, found: 471.0782 . Enantiomeric excess was found to be $86 \%$ by chiral HPLC (ChiralPak PC-1 column, methanol/water 9:1 $0.7 \mathrm{~mL} / \mathrm{min}, \mathrm{t}_{\text {major }}=18.52 \mathrm{~min}$, $\left.\mathrm{t}_{\text {minor }}=21.52 \mathrm{~min}\right)$.<smiles>C[C@H](Br)[C@]1(C)O[SiH](c2ccccc2)c2ccccc21</smiles>

(S)-3-((S)-1-bromoethyl)-1,3-dihydro-3-methyl-1,1-diphenylbenzo[c][1,2]oxasilole (2q): Following the general procedure, $\mathbf{2 q}$ was obtained from $\mathbf{1 q}$ (reaction time $6 \mathrm{~h}$ ) as colorless oil (43 $\mathrm{mg}, 91 \%$ ).

$\mathrm{R} f=0.5$ (petroleum ether/ethyl acetate $=35: 1) ;[\mathrm{a}]_{26.4}^{\mathrm{D}}=-21.2\left(\mathrm{c} 1.08, \mathrm{CHCl}_{3}\right) ; \mathrm{IR}(\mathrm{film}): 3003,2972$, 2937, 1426 1325, 1123, 1030, 907, 726, $516 \mathrm{~cm}^{-1} ;{ }^{1} \mathrm{H}$ NMR (400 MHz, $\left.\mathrm{CDCl}_{3}\right) \delta$ 7.84-7.70 (m, 3H), 7.59-7.55 (m, 2H), 7.52-7.33 (m, 9H), 4.50 (q, $J=7.0 \mathrm{~Hz}, 1 \mathrm{H}), 1.78$ (s, 3H), $1.77(\mathrm{~d}, J=7.0 \mathrm{~Hz}, 3 \mathrm{H})$. 
${ }^{13} \mathrm{C}$ NMR $\left(126 \mathrm{MHz}, \mathrm{CDCl}_{3}\right) \delta 155.77,135.45,135.01,134.93,133.25,132.59,132.04,130.47,130.35$, 130.33, 127.93, 127.86, 127.82, 122.73, 87.47, 58.54, 28.49, 21.30. HRMS-DART Positive calcd. for $\mathrm{C}_{22} \mathrm{H}_{22} \mathrm{BrOSi}[\mathrm{M}+\mathrm{H}]^{+} 409.0623$, found: 409.0623 . Enantiomeric excess was found to be $69 \%$ by chiral HPLC (ChiralPak AD-H column, hexane $/ \mathrm{i}-\mathrm{PrOH} 99: 10.7 \mathrm{~mL} / \mathrm{min}, \mathrm{t}_{\text {major }}=5.76 \mathrm{~min}, \mathrm{t}_{\text {minor }}=6.08 \mathrm{~min}$ ).<smiles>CCCC(Br)[C@]1(C)O[Si]([PH])(c2ccccc2)c2ccccc21</smiles>

$2 r$

(S)-3-((S)-1-bromobutyl)-1,3-dihydro-3-methyl-1,1-diphenylbenzo[c][1,2]oxasilole (2r): Following the general procedure, $2 \mathbf{r}$ was obtained from $\mathbf{r} \mathbf{r}$ (reaction time $10 \mathrm{~h}$ ) as colorless oil (40 $\mathrm{mg}, 93 \%$ ).

$\mathrm{R} f=0.5$ (petroleum ether/ethyl acetate $=40: 1) ;[\mathrm{a}]_{26.6}^{\mathrm{D}}=-102.7\left(\mathrm{c} 1.50, \mathrm{CHCl}_{3}\right) ; \mathrm{IR}($ film): 3006, 2974, 2935, $14231315,1113,1019,910,724,526 \mathrm{~cm}^{-1} ;{ }^{1} \mathrm{H}$ NMR (400 MHz, $\left.\mathrm{CDCl}_{3}\right) \delta$ 7.80-7.70 (m, 3H), 7.60-7.32 (m, 11H), $4.33(\mathrm{dd}, J=11.0,2.0 \mathrm{~Hz}, 1 \mathrm{H}), 1.97-1.88(\mathrm{~m}, 1 \mathrm{H}), 1.82(\mathrm{~s}, 3 \mathrm{H}), 1.76-1.64(\mathrm{~m}, 2 \mathrm{H})$, $1.39-1.30(\mathrm{~m}, 1 \mathrm{H}), 0.82(\mathrm{t}, J=7.0 \mathrm{~Hz}, 3 \mathrm{H}) .{ }^{13} \mathrm{C} \mathrm{NMR}\left(126 \mathrm{MHz}, \mathrm{CDCl}_{3}\right) \delta 155.94,135.45,135.00$, 134.90, 133.30, 132.55, 131.99, 130.49, 130.36, 130.33, 127.94, 127.82, 127.78, 123.02, 87.65, 65.63, 35.46, 29.54, 21.64, 13.31. HRMS-DART Positive calcd. for $\mathrm{C}_{24} \mathrm{H}_{26} \mathrm{BrOSi}[\mathrm{M}+\mathrm{H}]^{+}$437.0936, found: 437.0937. Enantiomeric excess was found to be $97 \%$ by chiral HPLC (ChiralPak PC-1 column, methanol/water $85: 150.7 \mathrm{~mL} / \mathrm{min}, \mathrm{t}_{\text {major }}=35.33 \mathrm{~min}, \mathrm{t}_{\text {minor }}=33.13 \mathrm{~min}$ ).<smiles>C[C@]1(C(Br)c2ccccc2)O[Si](c2ccccc2)(c2ccccc2)c2ccccc21</smiles>

(S)-3-((S)-bromo(phenyl)methyl)-1,3-dihydro-3-methyl-1,1-diphenylbenzo[c][1,2]oxasilole $\quad$ (2s): Following the general procedure, $2 \mathrm{~s}$ was obtained from $1 \mathrm{~s}$ (reaction time $24 \mathrm{~h}$ ) as colorless oil (41 $\mathrm{mg}$, $89 \%)$.

$\mathrm{R} f=0.5$ (petroleum ether/ethyl acetate $=25: 1) ;[\mathrm{a}]_{22.5}^{\mathrm{D}}=58.5\left(\right.$ c 0.55, $\left.\mathrm{CHCl}_{3}\right) ; \mathrm{IR}($ film): 3003, 2974, 2936, $14231310,1120,1030,907,726,527 \mathrm{~cm}^{-1} ;{ }^{1} \mathrm{H}$ NMR (500 MHz, $\left.\mathrm{CDCl}_{3}\right) \delta 7.80-7.73(\mathrm{~m}, 3 \mathrm{H})$, 7.58-7.33 (m, 13H), 7.27-7.23 (m, 3H), $5.27(\mathrm{~s}, 1 \mathrm{H}), 1.63(\mathrm{~s}, 3 \mathrm{H}) .{ }^{13} \mathrm{C}$ NMR $\left(126 \mathrm{MHz}, \mathrm{CDCl}_{3}\right) \delta$ $155.76,139.06,135.44,135.00,133.15,133.03,132.04,130.38,130.35,130.28,129.80,128.30$, 127.96, 127.90, 127.86, 127.77, 122.41, 87.67, 63.52, 28.81. HRMS-DART Positive calcd. for $\mathrm{C}_{27} \mathrm{H}_{24} \mathrm{BrOSi}[\mathrm{M}+\mathrm{H}]^{+}$471.0780, found: 471.0781. Enantiomeric excess was found to be $98.5 \%$ by chiral HPLC (ChiralPak PC-3 column, methanol/water 99:1 $0.7 \mathrm{~mL} / \mathrm{min}, \mathrm{t}_{\text {major }}=41.29 \mathrm{~min}, \mathrm{t}_{\text {minor }}=$ $22.17 \mathrm{~min})$.<smiles>[Z12][R]1(c2ccccc2)O[C@](C)(C(C)(C)Br)c2ccccc21</smiles>

(S)-3-(2-bromopropan-2-yl)-1,3-dihydro-3-methyl-1,1-diphenylbenzo[c][1,2]oxasilole

(2t): 
Following the general procedure, 2t was obtained from 1t (reaction time $12 \mathrm{~h}$ ) as colorless oil (34 $\mathrm{mg}$, $82 \%)$.

$\mathrm{R} f=0.5$ (petroleum ether/ ethyl acetate $=40: 1) ;[\mathrm{a}]_{26.5}^{\mathrm{D}}=-58.0\left(\right.$ ( $\left.0.28, \mathrm{CHCl}_{3}\right) ; \mathrm{IR}($ film): 3009, 2977, 2936, 1422, 1314, 1110, 1027, 903, 723, $517 \mathrm{~cm}^{-1}$; ${ }^{1} \mathrm{H}$ NMR (500 MHz, $\left.\mathrm{CDCl}_{3}\right) \delta 7.93$ (d, $J=8.0 \mathrm{~Hz}$, $1 \mathrm{H}), 7.77-7.73(\mathrm{~m}, 3 \mathrm{H})$, 7.51-7.47 (m, 4H), 7.46-7.31 (m, 6H), $1.98(\mathrm{~s}, 3 \mathrm{H}), 1.91(\mathrm{~s}, 3 \mathrm{H}), 1.53(\mathrm{~s}, 3 \mathrm{H})$. ${ }^{13} \mathrm{C}$ NMR $\left(126 \mathrm{MHz}, \mathrm{CDCl}_{3}\right) \delta 154.58,135.07,134.99,134.90,133.37,133.18,132.02,130.44,130.38$, 129.94, 128.02, 127.88, 127.84, 125.20, 90.33, 72.93, 30.93, 30.90, 27.86. HRMS-DART Positive calcd. for $\mathrm{C}_{23} \mathrm{H}_{24} \mathrm{BrOSi}[\mathrm{M}+\mathrm{H}]^{+} 423.0780$, found: 423.0780 . Enantiomeric excess was found to be $91 \%$ by chiral HPLC (ChiralPak PC-1 column, methanol/water $84: 160.7 \mathrm{~mL} / \mathrm{min}, \mathrm{t}_{\text {major }}=36.41 \mathrm{~min}, \mathrm{t}_{\text {minor }}=$ $34.01 \mathrm{~min})$.

\subsection{Derivatization of Benzoxasilole}

\section{Gram scale synthesis of 2a}

To a mixture of 8H-TRIP L1 (304 mg, $0.4 \mathrm{mmol})$, bromium salt B1 (2.78 g, $5.2 \mathrm{mmol})$, and $\mathrm{K}_{2} \mathrm{CO}_{3}$ $(2.21 \mathrm{~g}, 16 \mathrm{mmol})$ in anhydrous toluene $(40 \mathrm{~mL})$ was added dropwise a solution of substrate 1a (4 mmol) in toluene $(40 \mathrm{~mL})$ at $0{ }^{\circ} \mathrm{C}$ under argon atmosphere. The mixture was stirred at this temperature for $48 \mathrm{~h}$, at which time TLC showed the starting material still remained, and then another batch of B1 $(2.78 \mathrm{~g}, 5.2 \mathrm{mmol})$ was added and the reaction was stirred at the same temperature for additional $48 \mathrm{~h}$, at which time the starting material was consumed completely. The reaction mixture was filtered through a silica gel pad, washed with toluene, and concentrated in vacuum to afford the target product 2a as slightly yellow oil (1.48 g, 94\%) and it's pure enough for further usage. And enantiomeric excess was found to be $95 \%$ by chiral HPLC (ChiralPak OD-H column, hexane $/ i-\mathrm{PrOH}$ 99:1 $0.3 \mathrm{~mL} / \mathrm{min}, \mathrm{t}_{\text {major }}$ $\left.=14.93 \mathrm{~min}, \mathrm{t}_{\text {minor }}=15.75 \mathrm{~min}\right)$.<smiles>C[C@]1(CN)O[Si](c2ccccc2)(c2ccccc2)c2ccccc21</smiles>

To a mixture of 2a (39 mg, $0.1 \mathrm{mmol})$ and $\mathrm{NaN}_{3}(32 \mathrm{mg}, 0.5 \mathrm{mmol})$ under Ar was added DMF ( $\left.2 \mathrm{~mL}\right)$ and the mixture was stirred at $70{ }^{\circ} \mathrm{C}$ for $8 \mathrm{~h}$ and quenched by pouring into brine and the mixture was extracted with ethyl acetate. The combined organic phases were dried over $\mathrm{Na}_{2} \mathrm{SO}_{4}$, condensed and purified by preparative TLC to afford the target product 3a as colorless oil (32 $\mathrm{mg}, 89 \%$ ).

(S)-3-(azidomethyl)-1,3-dihydro-3-methyl-1,1-diphenylbenzo[c][1,2]oxasilole $\quad$ (3a): $\mathrm{R} f=0.5$ (petroleum ether/ethyl acetate $=30: 1)$; [a] ${ }_{23.0}^{\mathrm{D}}=21.3\left(\right.$ c 1.37, $\mathrm{CHCl}_{3}$ ); IR (film): 2973, 2937, 1423, 1317, 1115, 1026, 913, 729, $514 \mathrm{~cm}^{-1}$; ${ }^{1} \mathrm{H}$ NMR $\left(500 \mathrm{MHz}, \mathrm{CDCl}_{3}\right) \delta$ 7.75-7.71 (m, 1H), 7.71-7.67 (m, 2H), 7.64-7.60 (m, 2H), 7.50-7.35 (m, 8H), 7.28-7.25 (m, 1H), 3.55 (d, $J=12.5 \mathrm{~Hz}, 1 \mathrm{H}), 3.46$ (d, $J=$ $12.5 \mathrm{~Hz}, 1 \mathrm{H}), 1.68(\mathrm{~s}, 3 \mathrm{H}) .{ }^{13} \mathrm{C}$ NMR $\left(126 \mathrm{MHz}, \mathrm{CDCl}_{3}\right) \delta 154.38,135.08,134.96,133.51,132.83$, 132.37, 130.59, 130.53, 130.50, 128.07, 128.03, 128.00, 122.40, 85.87, 61.89, 27.42. HRMS-DART Positive calcd. for $\mathrm{C}_{21} \mathrm{H}_{20} \mathrm{~N}_{3} \mathrm{OSi}[\mathrm{M}+\mathrm{H}]^{+} 358.1376$, found: 356.1370 . Enantiomeric excess was found to be $92 \%$ by chiral HPLC (ChiralPak PA-2 column, hexane $/ \mathrm{i}-\mathrm{PrOH} 100: 0$ DIPEA $0.10 .7 \mathrm{~mL} / \mathrm{min}$, $\mathrm{t}_{\text {major }}=$ $\left.7.67 \mathrm{~min}, \mathrm{t}_{\mathrm{minor}}=6.98 \mathrm{~min}\right)$. 


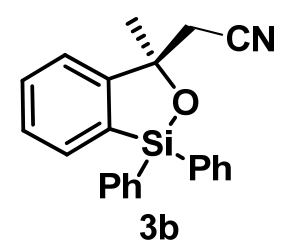

To a mixture of $2 \mathbf{a}(39 \mathrm{mg}, 0.1 \mathrm{mmol})$ and $\mathrm{NaCN}$ (4.6 mg, $0.095 \mathrm{mmol})$ under Ar was added DMF (2 $\mathrm{mL}$ ) and the mixture was stirred at $70{ }^{\circ} \mathrm{C}$ for $8 \mathrm{~h}$ and quenched by pouring into brine and the mixture was extracted with ethyl acetate. The combined organic phases were dried over $\mathrm{Na}_{2} \mathrm{SO}_{4}$, condensed and purified by preparative TLC to afford the target product $\mathbf{3 b}$ as colorless oil (27 $\mathrm{mg}, 82 \%)$.

2-((R)-1,3-dihydro-3-methyl-1,1-diphenylbenzo[c][1,2]oxasilol-3-yl)acetonitrile (3b): $\mathrm{R} f=0.5$ (petroleum ether/ethyl acetate $=20: 1) ;[\mathrm{a}]_{23.7}^{\mathrm{D}}=-2.2\left(\right.$ c $\left.0.85, \mathrm{CHCl}_{3}\right)$; IR (film): 2975, 2930, 1428, 1313, 1105, 1027, 910, 724, $508 \mathrm{~cm}^{-1} ;{ }^{1} \mathrm{H}$ NMR (400 MHz, $\left.\mathrm{CDCl}_{3}\right) \delta$ 7.78-7.35 (m, 14H), $2.88(\mathrm{dd}, J=$ $2.0 \mathrm{~Hz}, 1 \mathrm{H}), 2.84(\mathrm{dd}, J=2.0 \mathrm{~Hz}, 1 \mathrm{H}), 1.80(\mathrm{~s}, 3 \mathrm{H}) .{ }^{13} \mathrm{C} \mathrm{NMR}\left(126 \mathrm{MHz}, \mathrm{CDCl}_{3}\right) \delta 154.40,135.07$, $134.93,133.58,133.08,132.48,132.09,130.89,130.84,130.69,128.50,128.15,128.09,122.24$, 117.32, 82.71, 33.44, 29.56. HRMS-DART Positive calcd. for $\mathrm{C}_{22} \mathrm{H}_{20} \mathrm{NOSi}[\mathrm{M}+\mathrm{H}]^{+} 342.1314$, found: 342.1312. Enantiomeric excess was found to be $95 \%$ by chiral HPLC (ChiralPak OD-H column, hexane $/ \mathrm{i}-\mathrm{PrOH}$ 9:1 $\left.0.7 \mathrm{~mL} / \mathrm{min}, \mathrm{t}_{\text {major }}=9.30 \mathrm{~min}, \mathrm{t}_{\text {minor }}=10.14 \mathrm{~min}\right)$.<smiles>C[C@@](O)(CBr)c1ccccc1O</smiles>

4

4 was synthesized according to literature with little modification ${ }^{[5]}$.A mixture of $2 \mathbf{2 a}(39 \mathrm{mg}, 0.1 \mathrm{mmol})$, $\mathrm{KF}(29 \mathrm{mg}, 0.5 \mathrm{mmol})$ and $\mathrm{NaHCO}_{3}(29 \mathrm{mg}, 0.5 \mathrm{mmol})$ in $\mathrm{THF}(2 \mathrm{~mL})$ was cooled to $0{ }^{\circ} \mathrm{C}$ and $30 \%$ $\mathrm{H}_{2} \mathrm{O}_{2}(1 \mathrm{~mL})$. The mixture was allowed to warm to room temperature and stirred overnight. The reaction was directly purified by preparative TLC to afford the target product $\mathbf{4}$ as colorless oil (19 $\mathrm{mg}$, $85 \%)$.

2-((S)-1-bromo-2-hydroxypropan-2-yl)phenol (4): $\mathrm{R} f=0.5$ (petroleum ether/ethyl acetate $=4: 1$ ); $[\mathrm{a}]_{23.2}^{\mathrm{D}}=35.6$ (c 0.32, $\mathrm{CHCl}_{3}$ ); IR (film): 3387, 2975, 2936, 1425, 1310, 1106, 1024, 912, 728, 516 $\mathrm{cm}^{-1} ;{ }^{1} \mathrm{H}$ NMR $\left(500 \mathrm{MHz}, \mathrm{CDCl}_{3}\right) \delta$ 7.36-7.33 (m, 1H), 7.29-7.24 (m, 2H), $6.96(\mathrm{t}, J=7.5 \mathrm{~Hz}, 1 \mathrm{H})$, $6.88(\mathrm{~d}, J=8.0 \mathrm{~Hz}, 1 \mathrm{H}), 4.49$ (d, $J=10.0 \mathrm{~Hz}, 1 \mathrm{H}), 4.31$ (d, $J=10.0 \mathrm{~Hz}, 1 \mathrm{H}), 2.08(\mathrm{~s}, 1 \mathrm{H}), 1.69$ (s, 3H). ${ }^{13} \mathrm{C}$ NMR $\left(126 \mathrm{MHz}, \mathrm{CDCl}_{3}\right) \delta 159.76,131.91,130.30,122.93,121.05,110.67,84.01,77.92,24.89$. HRMS-DART Positive calcd. for $\mathrm{C}_{9} \mathrm{H}_{12} \mathrm{BrO}_{2}[\mathrm{M}+\mathrm{H}]^{+}$231.0021, found: 231.0031. Enantiomeric excess was found to be $99 \%$ by chiral HPLC (ChiralPak OD-H column, hexane/i-PrOH 9:1 0.7 mL/min, $\mathrm{t}_{\text {major }}$ $=9.23 \mathrm{~min}, \mathrm{t}_{\text {minor }}=8.43 \mathrm{~min}$ ).

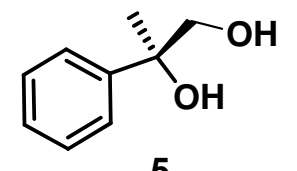

5

5 was synthesized according to literature ${ }^{[6]}$ and its absolute configuration was established to be $\mathrm{S}$ according literature ${ }^{[7,8]}$. The mixture of $\mathbf{2 a}(79 \mathrm{mg}, 0.2 \mathrm{mmol})$ and $\mathrm{KOH}(44 \mathrm{mg}, 0.8 \mathrm{mmol})$ in $\mathrm{EtOH} / \mathrm{H}_{2} \mathrm{O}(5 \mathrm{~mL}, \mathrm{v} / \mathrm{v}=4: 1)$ was stirred at $80{ }^{\circ} \mathrm{C}$ for $8 \mathrm{~h}$ and quenched by pouring into brine and the mixture was extracted with ethyl acetate. The combined organic phases were dried over $\mathrm{Na}_{2} \mathrm{SO}_{4}$, condensed and purified by preparative TLC to afford the target product 5 as colorless oil (27 mg, 90\%). (S)-2-phenylpropane-1,2-diol (5): $\mathrm{R} f=0.5$ (petroleum ether/ethyl acetate $=1: 1)$; $[\mathrm{a}]^{\mathrm{D}}{ }_{23.8}=11.4(c 0.40$, 
$\mathrm{CHCl}_{3}$ ); IR (film): 3450, 2975, 2937, 1423, 1314, 1109, 1024, 917, 720, $510 \mathrm{~cm}^{-1}$; ${ }^{1} \mathrm{H}$ NMR (400 MHz, $\left.\mathrm{CDCl}_{3}\right) \delta 7.44(\mathrm{~d}, J=7.5 \mathrm{~Hz}, 2 \mathrm{H}), 7.36(\mathrm{t}, J=7.5 \mathrm{~Hz}, 2 \mathrm{H}), 7.30-7.21(\mathrm{~m}, 1 \mathrm{H}), 3.77(\mathrm{~d}, J=11.0 \mathrm{~Hz}$, $1 \mathrm{H}), 3.61(\mathrm{~d}, J=11.0 \mathrm{~Hz}, 1 \mathrm{H}), 2.76$ (brs, $1 \mathrm{H}), 2.08$ (brs, $1 \mathrm{H}), 1.51(\mathrm{~s}, 3 \mathrm{H}) .{ }^{13} \mathrm{C} \mathrm{NMR}\left(126 \mathrm{MHz}, \mathrm{CDCl}_{3}\right)$ $\delta$ 144.94, 128.43, 127.19, 125.06, 74.86, 71.07, 26.02. HRMS-DART Positive calcd. for $\mathrm{C}_{9} \mathrm{H}_{13} \mathrm{O}_{2}$ $[\mathrm{M}+\mathrm{H}]^{+}$153.0916, found: 153.0913. Enantiomeric excess was found to be $95 \%$ by chiral HPLC (ChiralPak ID3 column, hexane/i-PrOH 9:1 $0.7 \mathrm{~mL} / \mathrm{min}, \mathrm{t}_{\text {major }}=7.54 \mathrm{~min}, \mathrm{t}_{\text {minor }}=9.86 \mathrm{~min}$ ).

\section{$2.5 \mathbf{m m o l}$ scale synthesis of $\mathbf{2 0}$}

To a mixture of 8H-TRIP L1 (190 mg, $0.25 \mathrm{mmol}$ ), bromium salt B1 (1.74 g, $3.25 \mathrm{mmol}$ ), and $\mathrm{K}_{2} \mathrm{CO}_{3}$ $(1.38 \mathrm{~g}, 10 \mathrm{mmol})$ in anhydrous toluene $(25 \mathrm{~mL})$ was added dropwise a solution of substrate $10(2.5$ mmol) in toluene $(25 \mathrm{~mL})$ at $0{ }^{\circ} \mathrm{C}$ under argon atmosphere. The mixture was stirred at this temperature for $36 \mathrm{~h}$, at which time TLC showed the starting material still remained, and then another batch of $\mathbf{B 1}$ ( $1.74 \mathrm{~g}, 3.25 \mathrm{mmol}$ ) was added and the reaction was stirred at the same temperature for additional $36 \mathrm{~h}$, at which time the starting material was consumed completely. The reaction mixture was filtered through a silica gel pad, washed with toluene, and concentrated in vacuum to afford the target product 20 as white solid (1.02 g, 94\%) and it's pure enough for further usage. And enantiomeric excess was found to be $89 \%$ by chiral HPLC (ChiralPak AD-H column, hexane $/ \mathrm{i}-\mathrm{PrOH}$ 100:0 $0.7 \mathrm{~mL} / \mathrm{min}$, $\mathrm{t}_{\text {major }}=$ $\left.8.51 \mathrm{~min}, \mathrm{t}_{\text {minor }}=7.09 \mathrm{~min}\right)$.

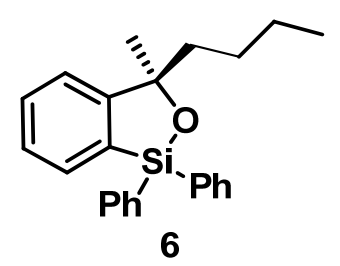

To a solution of $20(437 \mathrm{mg}, 1 \mathrm{mmol})$ and $(n-\mathrm{Bu})_{3} \mathrm{SnH}(436 \mathrm{mg}, 1.5 \mathrm{mmol})$ in toluene $(10 \mathrm{~mL})$ under Ar at $70{ }^{\circ} \mathrm{C}$ was added AIBN (16 mg, $0.1 \mathrm{mmol}$ ) in one portion and the mixture was stirred at the same temperature for $5 \mathrm{~h}$. The reaction was cooled to room temperature and condensed. The residue was purified by flash chromatography (petroleum ether/ethyl acetate $=400: 1$ ) to afford the target product as vicious oil (340 mg, 95\%).

(R)-3-butyl-1,3-dihydro-3-methyl-1,1-diphenylbenzo[c][1,2]oxasilole (6): $\mathrm{R} f=0.5$ (petroleum ether/ethyl acetate $=80: 1) ;[\mathrm{a}]_{26.2}^{\mathrm{D}}=-36.7\left(c 0.62, \mathrm{CHCl}_{3}\right)$; IR (film): 3006, 2975, 2937, 1423, 1314, 1109, 1024, 917, 720, $510 \mathrm{~cm}^{-1}$; ${ }^{1} \mathrm{H}$ NMR (500 MHz, $\left.\mathrm{CDCl}_{3}\right) \delta$ 7.79-7.63 (m, 5H), 7.53-7.35 (m, 8H), 7.32-7.28 (m, 1H), 2.01-1.95 (m, 1H), 1.95-1.87 (m, 1H), $1.67(\mathrm{~s}, 3 \mathrm{H}), 1.36-1.32(\mathrm{~m}, 1 \mathrm{H}), 1.27-1.20(\mathrm{~m}$, 2H), 1.12-1.05 (m, 1H), $0.79(\mathrm{t}, J=7.0 \mathrm{~Hz}, 3 \mathrm{H}) .{ }^{13} \mathrm{C}$ NMR $\left(126 \mathrm{MHz}, \mathrm{CDCl}_{3}\right) \delta 157.97,135.16,134.98$, 134.37, 132.18, 132.00, 130.31, 130.27, 130.23, 127.90, 127.84, 127.11, 122.39, 86.79, 43.44, 30.93, 26.47, 22.97, 13.94. HRMS-DART Positive calcd. for $\mathrm{C}_{24} \mathrm{H}_{27} \mathrm{OSi}[\mathrm{M}+\mathrm{H}]^{+}$359.1831, found: 359.1830 . Enantiomeric excess was found to be $90 \%$ by chiral HPLC (ChiralPak AD-H column, hexane/i-PrOH 1:0 $0.7 \mathrm{~mL} / \mathrm{min}, \mathrm{t}_{\text {major }}=7.07 \mathrm{~min}, \mathrm{t}_{\text {minor }}=7.05 \mathrm{~min}$ ).<smiles>[3H][PH](c1ccccc1)(c1ccccc1)c1ccccc1[Si](C)(c1ccccc1)C(C)(O)C[P+]#CC</smiles> 
7 was synthesized according to literature with little modification ${ }^{[9]}$. A solution of 6 (144 mg, $\left.0.4 \mathrm{mmol}\right)$ $2 \mathrm{~mL}$ THF was cooled to $-78{ }^{\circ} \mathrm{C}$ under Ar, and MeLi (1.2 eq) was added dropwise. The mixture was stirred for $2 \mathrm{~h}$ at the same temperature and quenched by adding $2 \mathrm{M}$ aq. $\mathrm{H}_{2} \mathrm{SO}_{4}$. The resulting mixture was extracted with ethyl acetate. The organic phases were combined, dried over $\mathrm{Na}_{2} \mathrm{SO}_{4}$, and concentrated. The crude product was purified by preparative TLC to afford the target product, as white solid (140 mg, 95\%).

(R)-2-(2-(methyldiphenylsilyl)phenyl)hexan-2-ol (7): $\mathrm{R} f=0.5$ (petroleum ether/ethyl acetate $=40: 1$ ); $[\mathrm{a}]_{26.6}^{\mathrm{D}}=-26.0$ (c 1.00, $\mathrm{CHCl}_{3}$ ); IR (film): 3404, 2975, 2937, 2827, 1425, 1313, 1120, 1015, 914, 723, $527 \mathrm{~cm}^{-1} ;{ }^{1} \mathrm{H}$ NMR $\left(500 \mathrm{MHz}, \mathrm{CDCl}_{3}\right) \delta$ 7.55-7.51 (m, 4H), 7.48-7.31 (m, 8H), 7.30-7.27 (m, 1H), 7.17 $(\mathrm{td}, J=7.5,1.0 \mathrm{~Hz}, 1 \mathrm{H}), 1.97-1.90(\mathrm{~m}, 1 \mathrm{H}), 1.71-1.65(\mathrm{~m}, 1 \mathrm{H}), 1.49(\mathrm{~s}, 3 \mathrm{H}), 1.28-1.21(\mathrm{~m}, 2 \mathrm{H})$, $1.12-1.03(\mathrm{~m}, 2 \mathrm{H}), 1.05(\mathrm{~s}, 3 \mathrm{H}), 0.87(\mathrm{t}, J=7.5 \mathrm{~Hz}, 3 \mathrm{H}) .{ }^{13} \mathrm{C} \mathrm{NMR}\left(126 \mathrm{MHz}, \mathrm{CDCl}_{3}\right) \delta 155.37,141.32$, $141.13,139.33,134.75,134.70,131.65,129.45,128.51,128.47,127.83,125.79,125.56,77.43,44.24$, 31.67, 25.95, 22.99, 14.03, 0.80. HRMS-DART Positive calcd. for $\mathrm{C}_{25} \mathrm{H}_{31} \mathrm{OSi}[\mathrm{M}+\mathrm{H}]^{+} 375.2144$, found: 375.2147. Enantiomeric excess was found to be $89 \%$ by chiral HPLC (ChiralPak PC-3 column, methanol/water $85: 150.7 \mathrm{~mL} / \mathrm{min}, \mathrm{t}_{\text {major }}=17.47 \mathrm{~min}, \mathrm{t}_{\text {minor }}=23.02 \mathrm{~min}$ ). 
Table S6. Optimization of reaction condition for selective transfer of phenyl.

\begin{tabular}{|c|c|c|c|c|c|c|c|c|c|}
\hline entry & solvent & $\begin{array}{c}\text { cat. } \\
\text { (equiv) }\end{array}$ & $\begin{array}{c}\text { base } \\
\text { (equiv) }\end{array}$ & $\begin{array}{l}\text { ligand } \\
\text { (equiv) }\end{array}$ & $\begin{array}{l}\mathrm{T} \\
{ }^{\circ} \mathrm{C}\end{array}$ & $\begin{array}{c}\mathrm{t} \\
(\mathrm{h})\end{array}$ & $\begin{array}{l}\text { yield } \\
(\%)^{[c]}\end{array}$ & $8: 6^{[d]}$ & $\begin{array}{l}\mathrm{dr} \text { of } \\
8^{\text {[d] }}\end{array}$ \\
\hline $1^{[\mathrm{a}]}$ & THF & $\begin{array}{c}{\left[\mathrm{Rh}(\mathrm{OH})(\mathrm{coe})_{2}\right]_{2}} \\
(0.1)\end{array}$ & -- & L1 (0.15) & 65 & 12 & 85 & $1: 17$ & -- \\
\hline $2^{[b]}$ & DMSO & $\mathrm{PdCl}_{2}(0.1)$ & $\mathrm{K}_{2} \mathrm{CO}_{3}(2.0)$ & L2 (0.15) & 65 & 10 & 73 & $1: 1.5$ & $1.3: 1$ \\
\hline $3^{[\mathrm{b}]}$ & DMSO & $\begin{array}{c}\mathrm{PdCl}_{2}(0.1) \\
\mathrm{CuI}(0.3)\end{array}$ & $\mathrm{K}_{2} \mathrm{CO}_{3}(2.0)$ & L2 (0.15) & 65 & 10 & 54 & $1: 0$ & $1.3: 1$ \\
\hline $4^{[f]}$ & DMSO & $\mathrm{CuI}(0.3)$ & $\mathrm{K}_{2} \mathrm{CO}_{3}(2.0)$ & -- & 65 & 1.5 & 62 & $1: 0$ & $1.3: 1$ \\
\hline 5 & DMSO & $\mathrm{CuI}(0.3)$ & -- & -- & 65 & 5 & 62 & $1: 0$ & $1.3: 1$ \\
\hline 6 & THF & $\mathrm{CuI}(0.3)$ & -- & -- & 65 & 5 & 85 & $1: 0$ & $1.3: 1$ \\
\hline 7 & THF & $\mathrm{CuI}(0.2)$ & -- & -- & 65 & 5 & 86 & $1: 0$ & $1.3: 1$ \\
\hline 8 & THF & $\mathrm{CuI}(0.1)$ & -- & -- & 65 & 8 & 75 & $1: 0$ & $1.3: 1$ \\
\hline 9 & THF & -- & -- & -- & 65 & 24 & 0 & -- & -- \\
\hline 10 & THF & $\mathrm{CuI}(0.2)$ & -- & -- & 40 & 8 & 89 & $1: 0$ & $1.3: 1$ \\
\hline 11 & THF & $\mathrm{CuI}(0.2)$ & -- & -- & 20 & 24 & 23 & $1: 0$ & $1.3: 1$ \\
\hline 12 & THF & $\mathrm{CuBr}(0.2)$ & -- & -- & 40 & 8 & 58 & $1: 0$ & $1.3: 1$ \\
\hline 13 & THF & $\mathrm{CuCl}(0.2)$ & -- & -- & 40 & 8 & 63 & $1: 0$ & $1.3: 1$ \\
\hline 14 & THF & $\mathrm{Cu}(\mathrm{OTf})_{2}(0.2)$ & -- & -- & 40 & 24 & $0^{[\mathrm{ee}]}$ & -- & -- \\
\hline 15 & THF & $\mathrm{Cu}(\mathrm{OAc})_{2}(0.2)$ & -- & -- & 40 & 8 & 91 & $1: 0$ & $1.3: 1$ \\
\hline 16 & THF & $\mathrm{Cu}(\mathrm{OAc})_{2}(0.2)$ & -- & $\mathbf{L 1}(0.25)$ & 40 & 8 & 91 & $1: 0$ & $1.3: 1$ \\
\hline 17 & THF & $\mathrm{Cu}(\mathrm{OAc})_{2}(0.2)$ & -- & L3 (0.25) & 40 & 8 & 88 & $1: 0$ & $1.3: 1$ \\
\hline 18 & THF & $\mathrm{Cu}(\mathrm{OAc})_{2}(0.2)$ & -- & L4 (0.25) & 40 & 8 & 43 & $1: 0$ & $1.3: 1$ \\
\hline 19 & THF & $\mathrm{Cu}(\mathrm{OAc})_{2}(0.2)$ & -- & L5 (0.25) & 40 & 8 & 64 & $1: 0$ & $1.3: 1$ \\
\hline $20^{[\mathrm{g}]}$ & THF & $\mathrm{Cu}(\mathrm{OAc})_{2}(0.2)$ & -- & L6 $(0.25)$ & 40 & 8 & 87 & $1: 0$ & $1.3: 1$ \\
\hline $21^{[\mathrm{g}]}$ & THF & $\mathrm{Cu}(\mathrm{OAc})_{2}(0.2)$ & -- & L7 (0.25) & 40 & 8 & 90 & $1: 0$ & $1.3: 1$ \\
\hline $22^{[\mathrm{g}]}$ & THF & $\mathrm{Cu}(\mathrm{OAc})_{2}(0.2)$ & -- & L8 $(0.25)$ & 40 & 8 & 72 & $1: 0$ & $1.3: 1$ \\
\hline
\end{tabular}

[a] A mixture of $\left[\mathrm{Rh}(\mathrm{OH})(\mathrm{coe})_{2}\right]_{2}(0.01 \mathrm{mmol}), \mathbf{L 1}(0.015 \mathrm{mmol})$ in THF $(0.5 \mathrm{~mL})$ was stirred for $20 \mathrm{~min}$ at $\mathrm{rt}$, then was added $7(1.0 \mathrm{~mol})$ and methyl acrylate $(0.12 \mathrm{~mol})$ in THF $(0.5 \mathrm{~mL})$ under Ar and stirred at $65^{\circ} \mathrm{C}$. [b] A mixture of $7\left(1.0 \mathrm{~mol}, \mathrm{PhI}(0.12 \mathrm{~mol}), \mathrm{PdCl}_{2}(0.01 \mathrm{mmol}), \mathbf{L} 2(0.15 \mathrm{mmol})\right.$ and $\mathrm{K}_{2} \mathrm{CO}_{3}(0.2 \mathrm{mmol})$ in DMSO $(1 \mathrm{~mL})$ was heated to $65^{\circ} \mathrm{C}$. [c] isolated yield. [d] determined by $1 \mathrm{H}$ NMR. [f] the reaction was carried out in the absence of PhI and Palladium catalyst. L1 $=$ $(S, S)$-Me-Duphos, $\mathbf{L} 2=(2 \text {-furyl })_{3} \mathrm{P}, \mathbf{L 3}=(S)$-Tol-BINAP, L5 = 2,2'-Dipyridyl, L8 $=(R, R)$-DIPAMP<smiles>CC(C)c1cccc(C(C)C)c1N1CCN(c2c(C(C)C)cccc2C(C)C)C1</smiles><smiles>CC(C)(C1=N[C@H](C(C)(C)C)CO1)C1=N[C@H](C(C)(C)C)CO1</smiles>

L6<smiles>O=C(NC1CCCCC1NC(=O)c1ccccn1)c1ccccn1</smiles>

L7 


\section{Optimal reaction condition}

To a mixture of $7(0.1 \mathrm{mmol})$ and $\mathrm{Cu}(\mathrm{OAc})_{2}(0.02 \mathrm{mmol})$ under air was added THF $(2 \mathrm{~mL})$. The mixture was stirred at $40{ }^{\circ} \mathrm{C}$ for $8 \mathrm{~h}$. The reaction was directly purified by preparative TLC to afford the target product 8 , as colorless oil ( $27 \mathrm{mg}, 91 \%)$, as a mixture of two isomers $(\mathrm{dr}=1.3: 1$ determined by ${ }^{1} \mathrm{H}$ NMR). Biphenyl was also identified on ${ }^{1} \mathrm{H}$ NMR spectrum of crude mixture (60\% by using 2,4-dimethoxybenzene as inner standard).

(3R)-3-butyl-1,3-dihydro-1,3-dimethyl-1-phenylbenzo[c][1,2]oxasilole (8): $\mathrm{R} f=0.5$ (petroleum ether/ethyl acetate $=80: 1) ;[\mathrm{a}]_{26.5}^{\mathrm{D}}=-10.7\left(c 0.75, \mathrm{CHCl}_{3}\right)$; IR (film): 3404, 2974, 2933, 2822, 1425, 1316, 1129, 1012, 910, 727, $517 \mathrm{~cm}^{-1}$; For minor isomer: ${ }^{1} \mathrm{H} \mathrm{NMR}\left(500 \mathrm{MHz}, \mathrm{CDCl}_{3}\right) \delta$ 7.64-7.57 (m, $3 \mathrm{H})$, 7.48-7.31 (m, 5H), 7.28-7.21 (m, 1H), 2.05-1.72 (m, 3H), $1.60(\mathrm{~s}, 3 \mathrm{H}), 1.49-1.16(\mathrm{~m}, 4 \mathrm{H})$, 1.11-0.97 (m, 1H), $0.78(\mathrm{t}, J=7.5 \mathrm{~Hz}, 3 \mathrm{H}), 0.70(\mathrm{~s}, 3 \mathrm{H}) .{ }^{13} \mathrm{C}_{\mathrm{NMR}}\left(126 \mathrm{MHz}, \mathrm{CDCl}_{3}\right) \delta 157.86,136.38$, 134.14, 133.33, 131.53, 130.09, 127.83, 126.92, 122.30, 86.28, 43.34, 31.05, 26.45, 22.96, 13.92, -0.37. Enantiomeric excess was found to be $87 \%$ by chiral HPLC (ChiralPak PC-3 column, methanol/water $\left.85: 150.7 \mathrm{~mL} / \mathrm{min}, \mathrm{t}_{\text {major }}=12.00 \mathrm{~min}, \mathrm{t}_{\text {minor }}=14.24 \mathrm{~min}\right)$. For the major isomer: ${ }^{1} \mathrm{H} \mathrm{NMR}(500 \mathrm{MHz}$, $\left.\mathrm{CDCl}_{3}\right) \delta$ 7.64-7.57 (m, 3H), 7.48-7.31 (m, 5H), 7.28-7.21 (m, 1H), 2.05-1.72 (m, 3H), $1.59(\mathrm{~s}, 3 \mathrm{H})$, 1.49-1.16 (m, 4H), 1.11-0.97 (m, 1H), $0.88(\mathrm{t}, J=7.0 \mathrm{~Hz}, 3 \mathrm{H}), 0.73$ (s, 3H). ${ }^{13} \mathrm{C}$ NMR $(126 \mathrm{MHz}$, $\left.\mathrm{CDCl}_{3}\right) \delta 157.21,136.97,133.94,133.69,131.33,130.09,130.03,127.92,122.20,86.43,43.42,31.43$, $26.34,22.98,14.04,-1.36$. Enantiomeric excess was found to be $87 \%$ for the minor isomer and $85 \%$ for the major isomer by chiral HPLC (ChiralPak PC-3 column, methanol/water $85: 150.7 \mathrm{~mL} / \mathrm{min}, \mathrm{t}_{\text {major }}=$ $23.32 \mathrm{~min}, \mathrm{t}_{\text {minor }}=16.59 \mathrm{~min}$ ).

HRMS-DART Positive calcd. for $\mathrm{C}_{19} \mathrm{H}_{25} \mathrm{OSi}[\mathrm{M}+\mathrm{H}]^{+}$297.1675, found: 297.1677.

Biphenyl: ${ }^{1} \mathrm{H}$ NMR $\left(500 \mathrm{MHz}, \mathrm{CDCl}_{3}\right) \delta 7.65(\mathrm{~d}, J=12.0 \mathrm{~Hz}, 2 \mathrm{H}), 7.50(\mathrm{t}, J=12.5 \mathrm{~Hz}, 2 \mathrm{H}), 7.40(\mathrm{t}, J$ $=12.0 \mathrm{~Hz}, 2 \mathrm{H}){ }^{13} \mathrm{C}$ NMR $\left(126 \mathrm{MHz}, \mathrm{CDCl}_{3}\right) \delta 141.30,128.81,127.30,127.22$. 


\section{References}

[1] W. Xie, G. Jiang, H. Liu, J. Hu, X. Pan, H. Zhang, X. Wan, Y. Lai, D. Ma, Angew. Chem. 2013, 125, $1-5$.

[2] H. Liu, G. Jiang, X. Pan, X. Wan, Y. Lai, D. Ma, W. Xie, Org. Lett. 2014, 16, 1908.

[3] J. Kim, H. B. Li, A. S. Rosenthal, D. Sang, T. A. Shapiro, M. D. Bachi, G. H. Posner, Tetrahedron, 2006, 62, 4120-4127.

[4] G. Rouquet, F. Robert, R. Méreau, F. Castet, P. Renaud, Y. Landais, Chem.-Eur. J., 2012, 18, 940-950.

[5] J. A. Marshall, M. M. Yanik, J. Org. Chem. 2001, 66, 1373-1379.

[6] M. Schlosser, C. Heiss, Eur. J. Org. Chem. 2003, 4618-4624.

[7] C. Gaul, K. Scharer, D. Seebach, J. Org. Chem. 2001, 66, 3059-3073.

[8] K. Toribatake, H. Nishiyama, Angew. Chem.Int. Ed. 2013, 52, 11011-11015.

[9] Y. Nakao, M. Takeda, T. Matsumoto, T. Hiyama, Angew. Chem.Int. Ed. 2010, 49, 4447-4450.

[10] R. Shintani, E. E. Maciver, F. Tamakuni, T. Hayashi, J. Am. Chem. Soc. 2012, 134, 16955-16958.

[11] Y. Nakao, H. Imanaka, J. Chen, A. Yada, T. Hiyama J. Organometal. Chem. 2007, 692, 583-603.

[12] Y. Nakao, H. Imanada, A. K. Sahoo, A. Yada, T. Hiyama, J. Am. Chem. Soc. 2005, 127, 6952-6953. 


\section{Plausible catalytic cycle and transition sate}

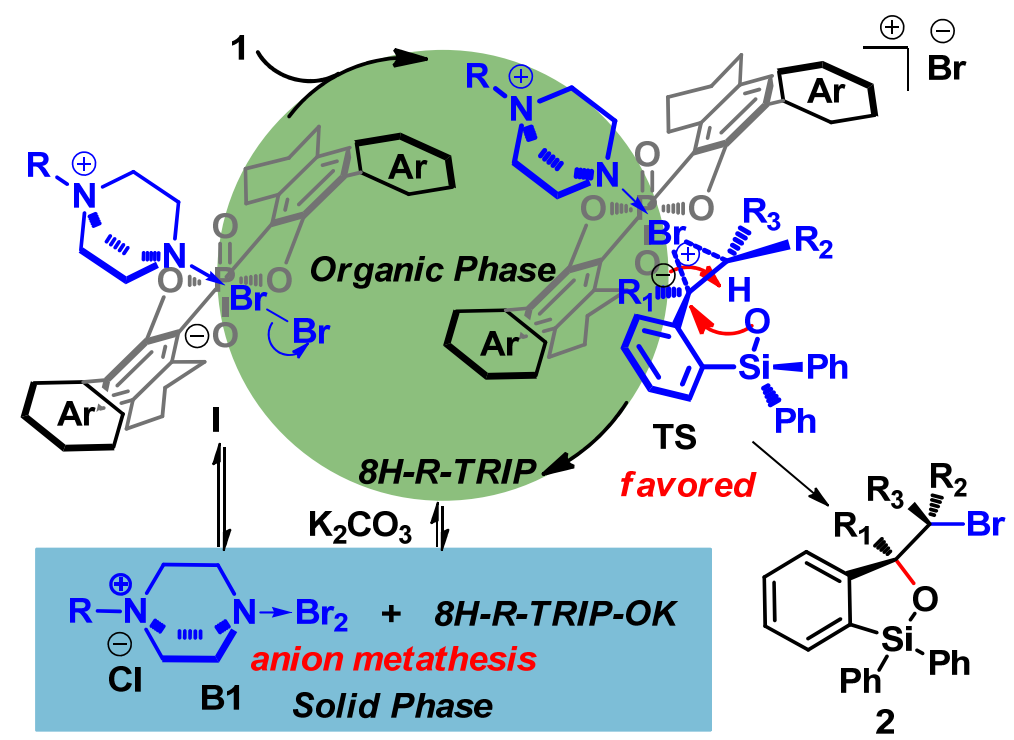

Figure S2. Plausible catalytic cycle of asymmetric bromo-oxycyclization of silanol.

Based on Toste's working model, the catalytic cycle of this reaction is proposed to begin with formation of potassium phosphate from $8 \mathrm{H}-\mathrm{R}$-TRIP and $\mathrm{K}_{2} \mathrm{CO}_{3}$, which migrates into solid phase (Figure S2). Anion metathesis of B1 and potassium phosphate in solid phase leads to a new bromine complex I, which enters into organic phase with the assistance of soluble phosphate. At this conjunction, a putative bromium is generated with heterolytic cleavage of bromine, which complexes with alkene of silanol 1 and undergoes 5-exo cyclization to deliver benzosilole 2 with concurrent regeneration of $8 \mathrm{H}-\mathrm{R}$-TRIP. In the energetically favored transition state TS, bromonium intermediate is stabilized by the DABCO ammonium moiety and simultaneously the phosphate interacts with silanol though hydrogen bond, which leads to a well-defined transition state. It's also proposed that the bulky silane moiety points outward the chiral pocket of chiral phosphate, thus favorably producing $S$ isomer. In the scenario, $\mathrm{R}_{1}$ and $\mathrm{R}_{3}$ are sensitive to steric hindrance due to their proximity to phosphate scaffold, while bulky groups are tolerated on $\mathrm{R}_{2}$ as this substituent points toward outside of the chiral pocket of phosphate, which is consistent with observed results. 
Copies of NMR spectra of new compounds
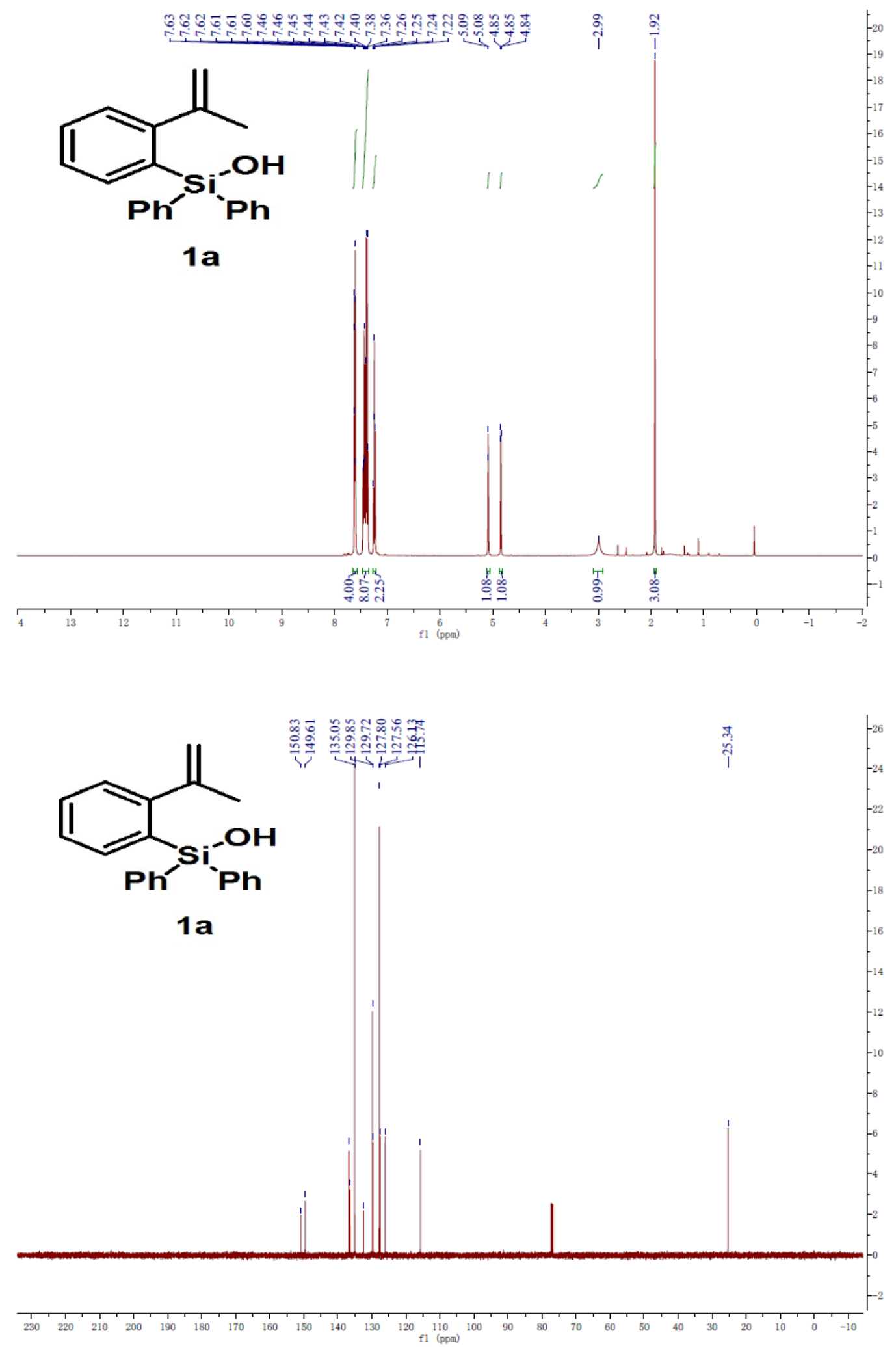

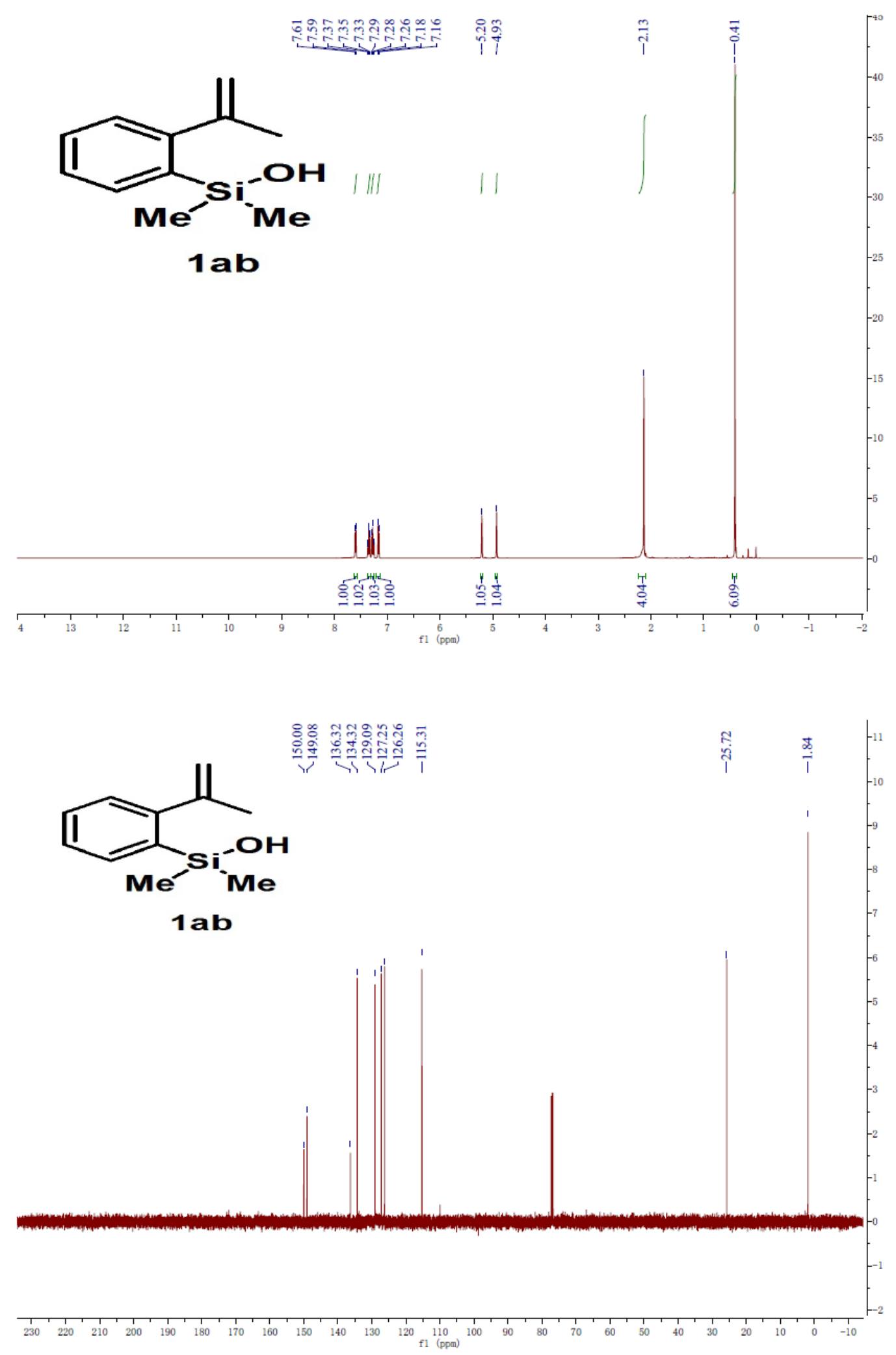

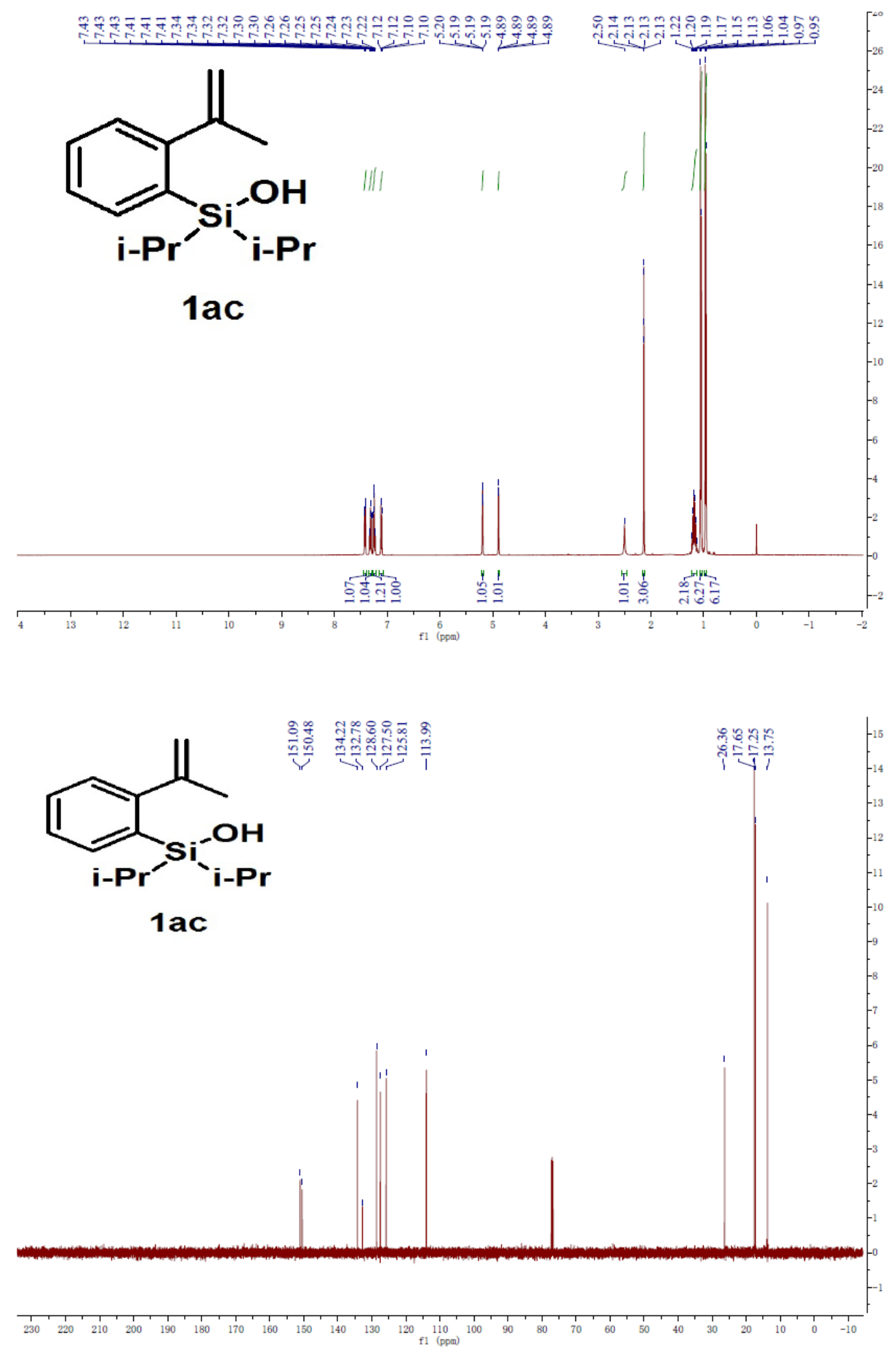

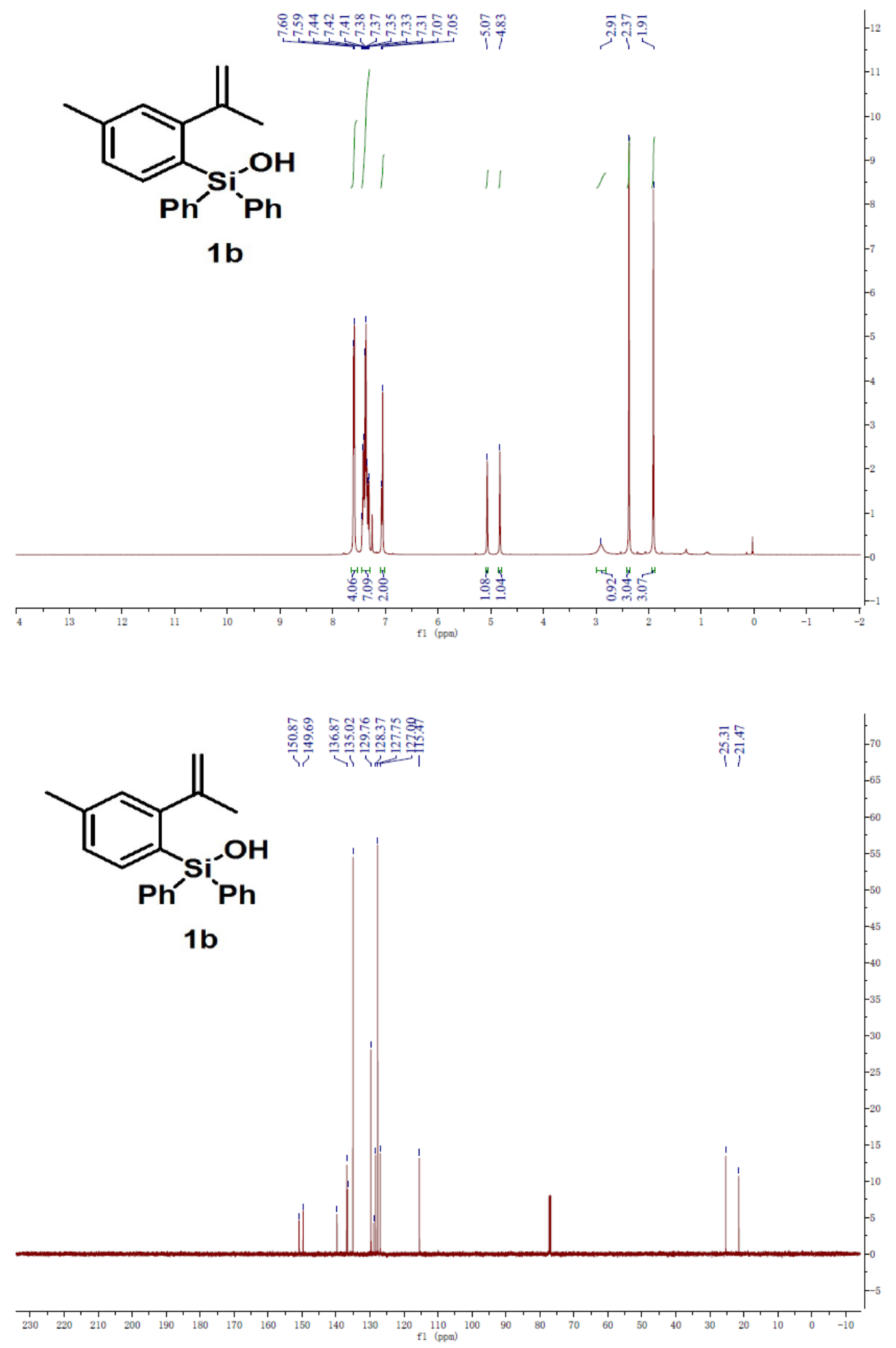

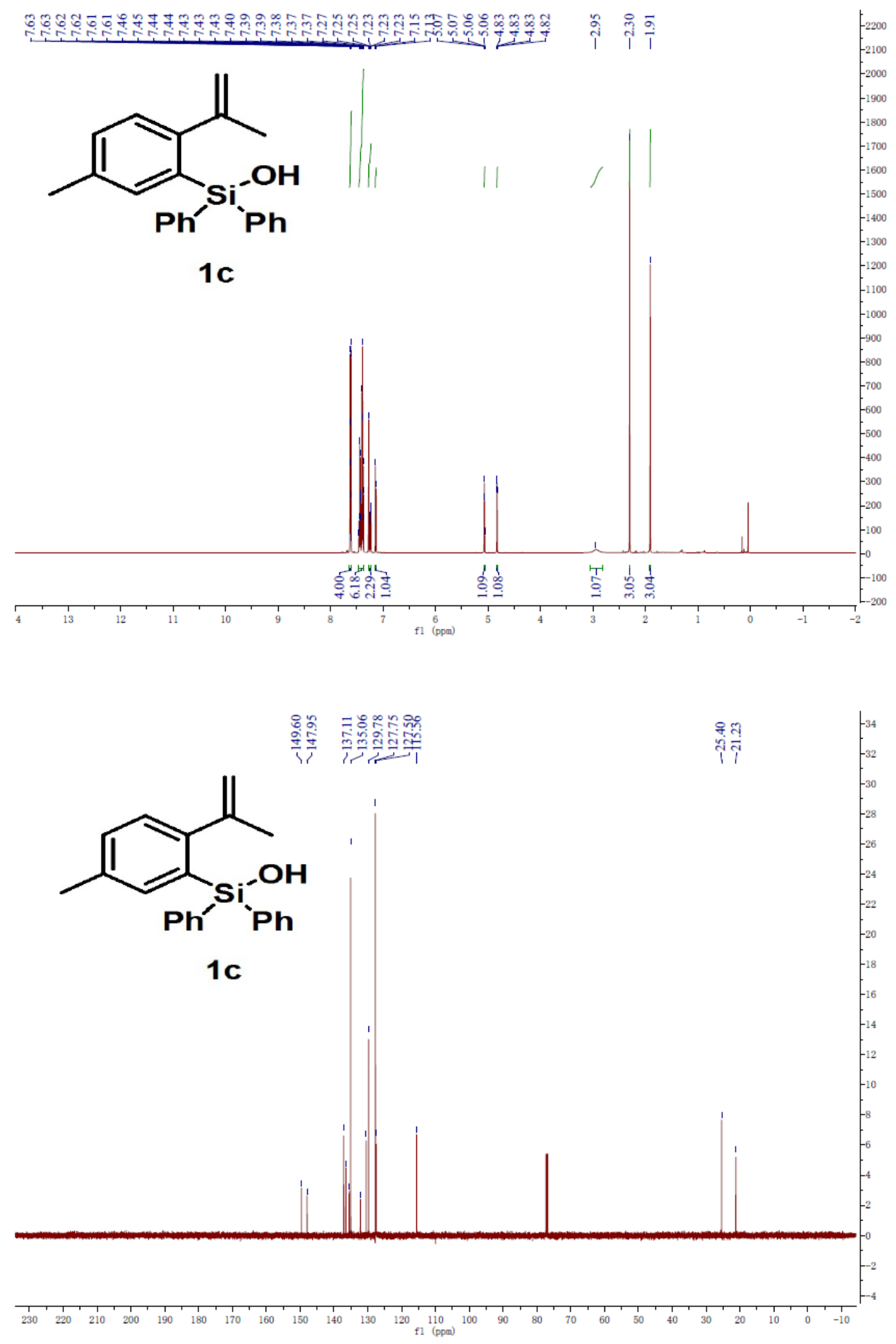

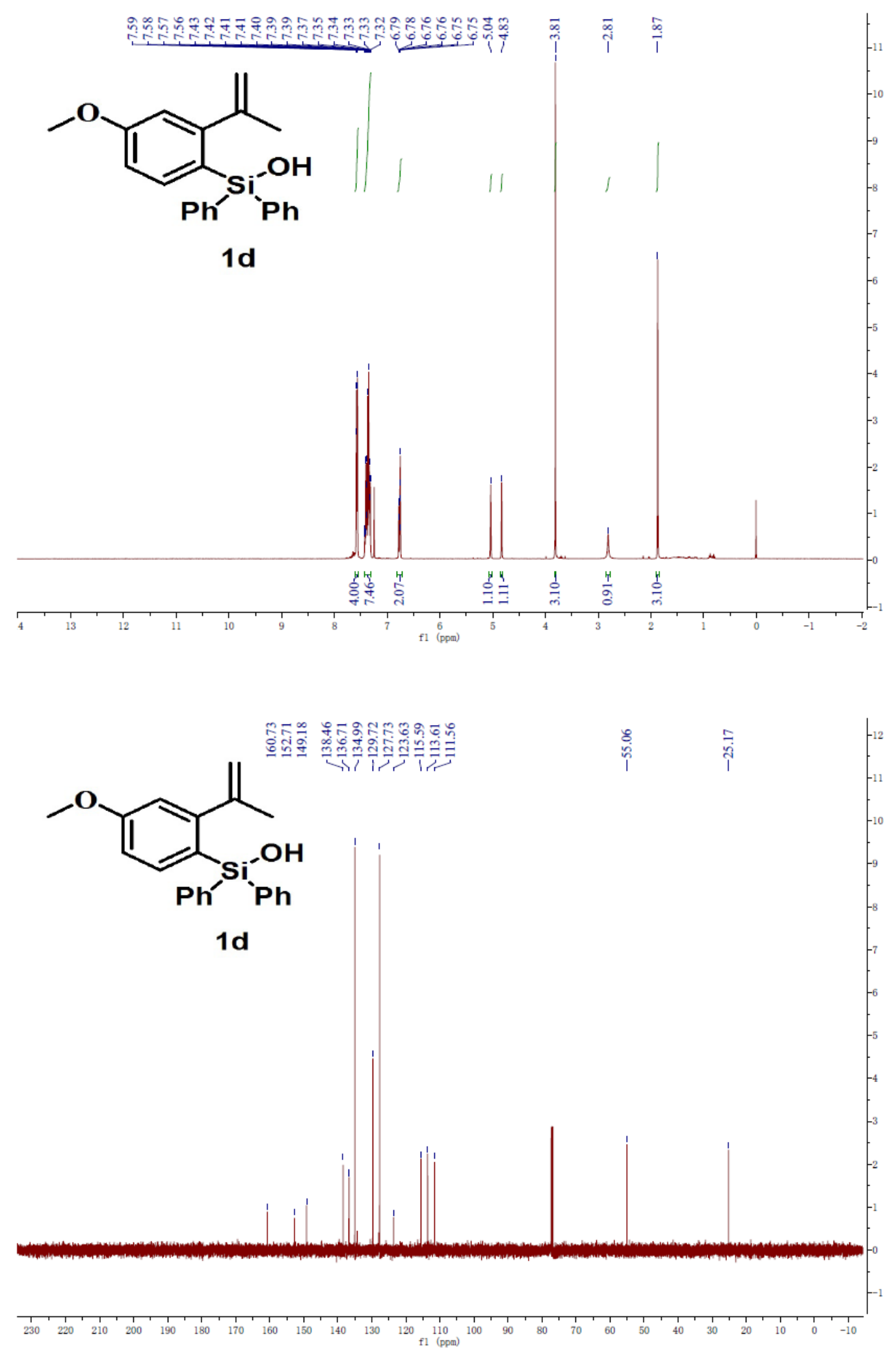

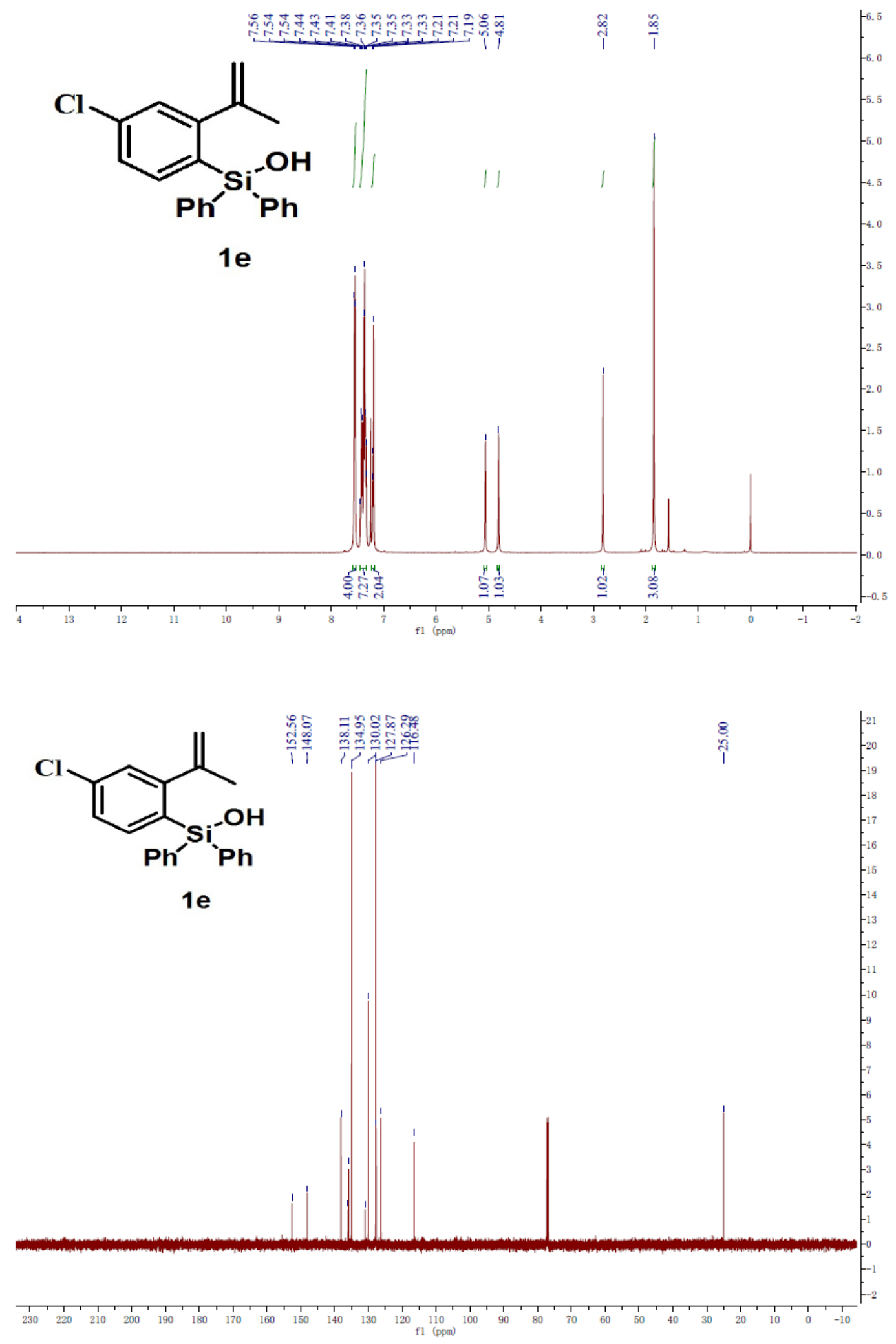

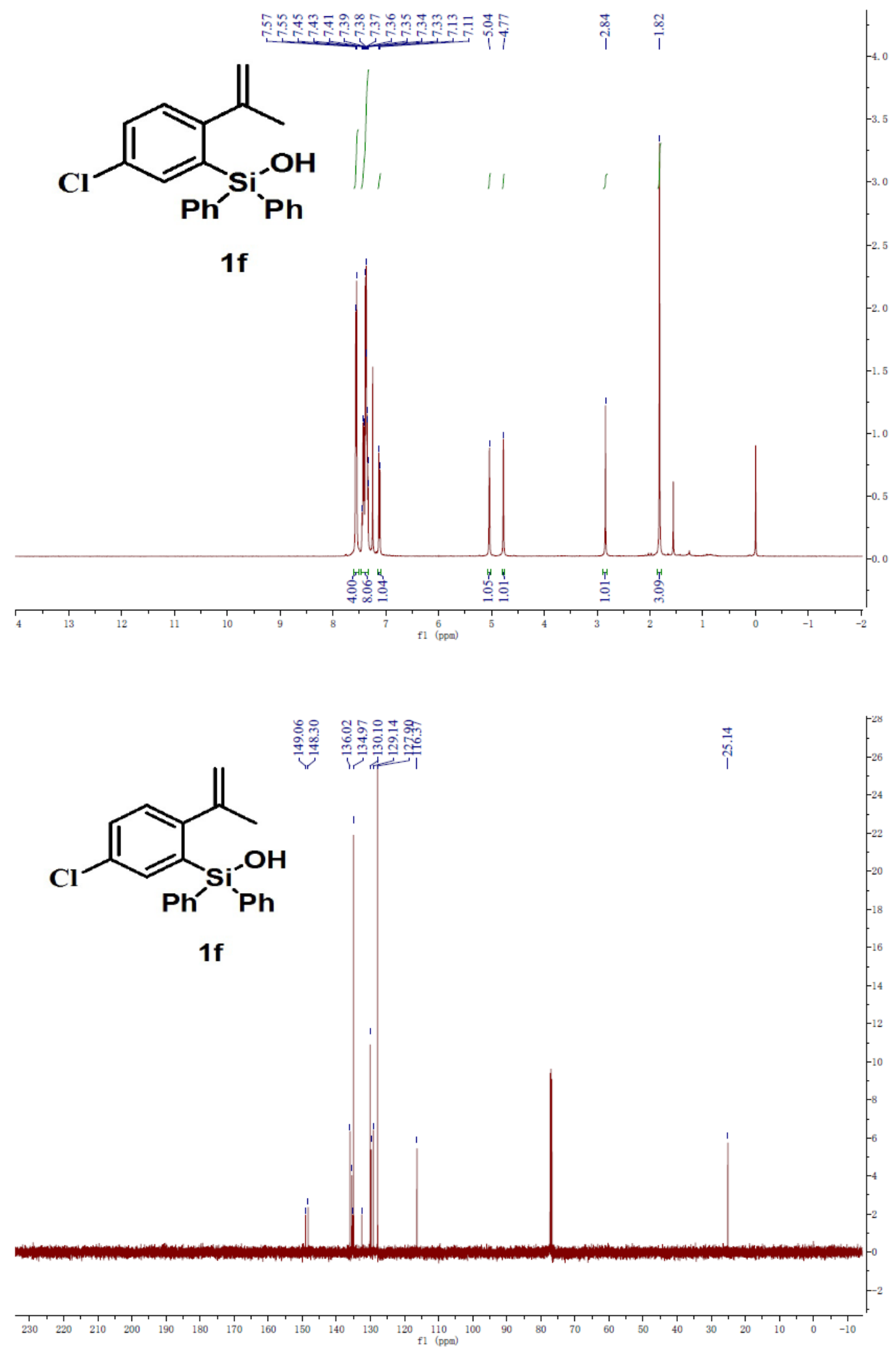

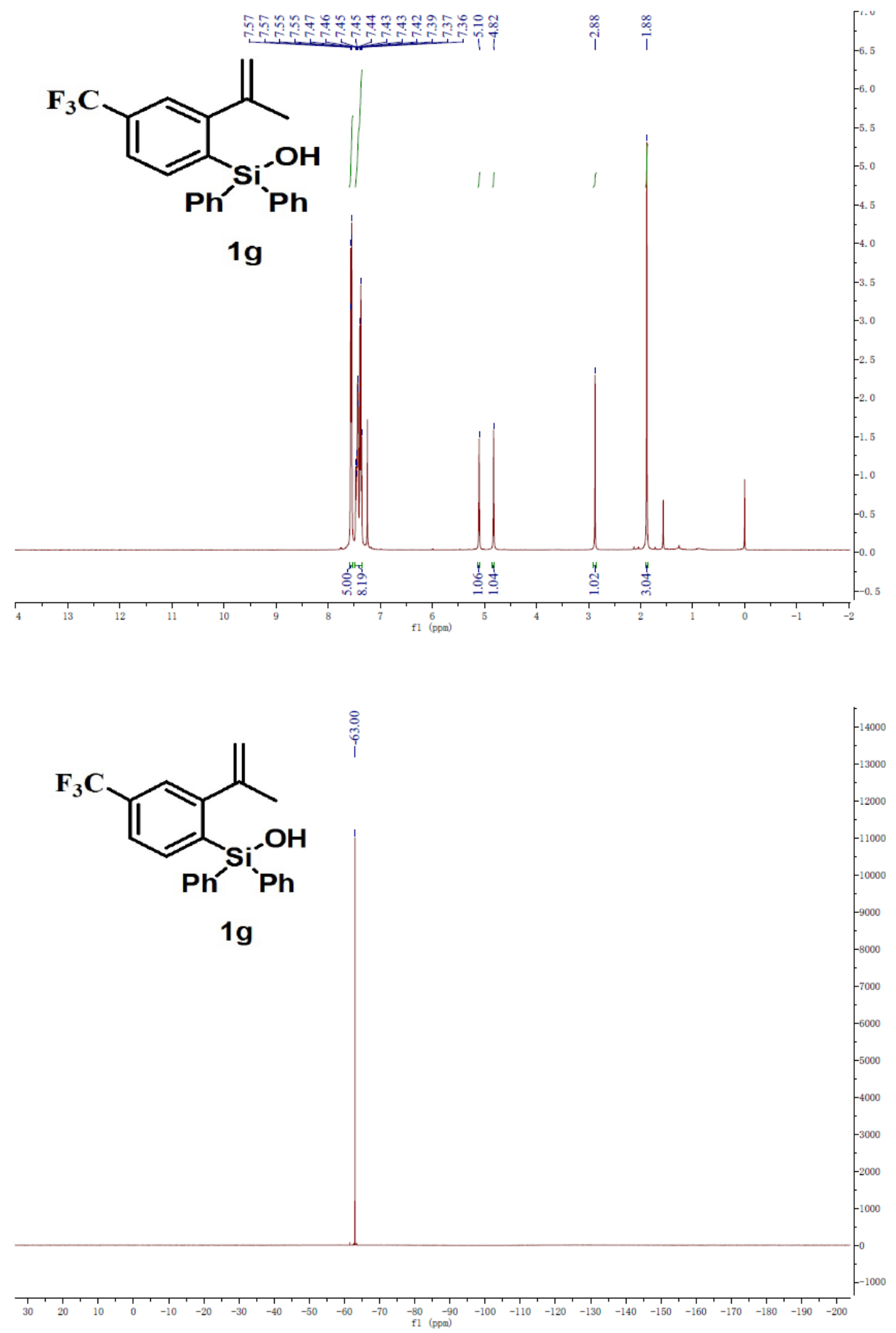

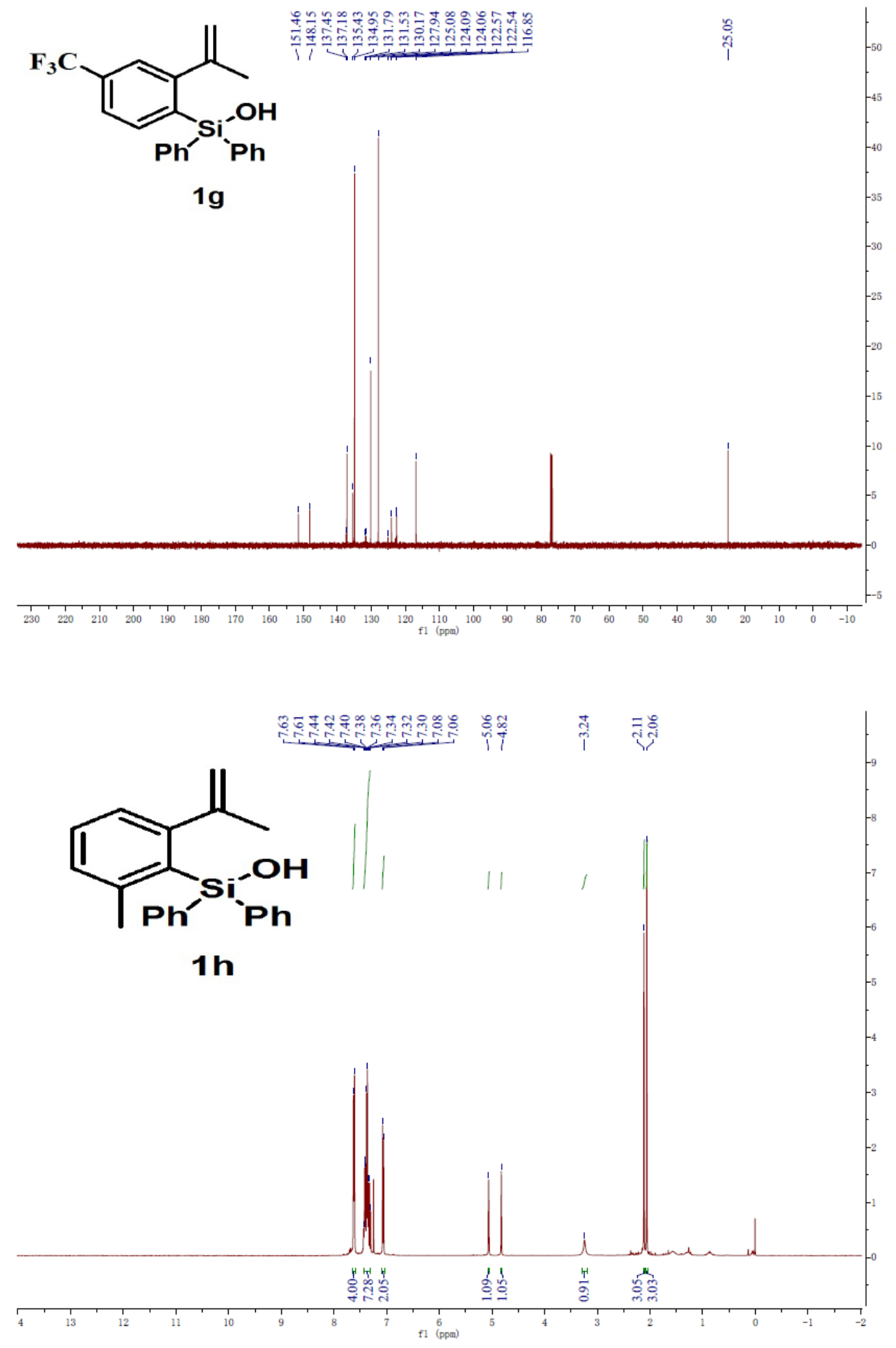

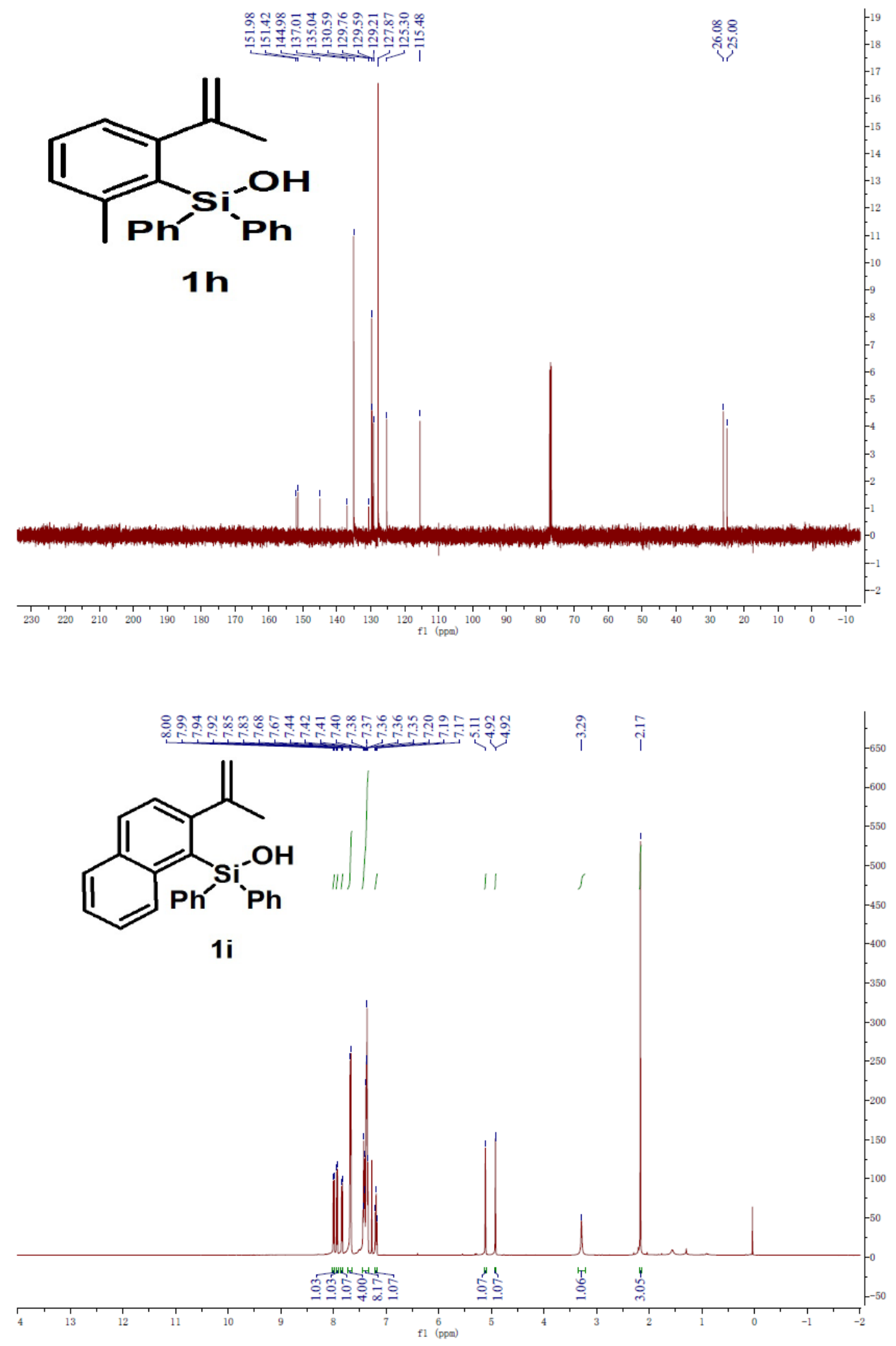

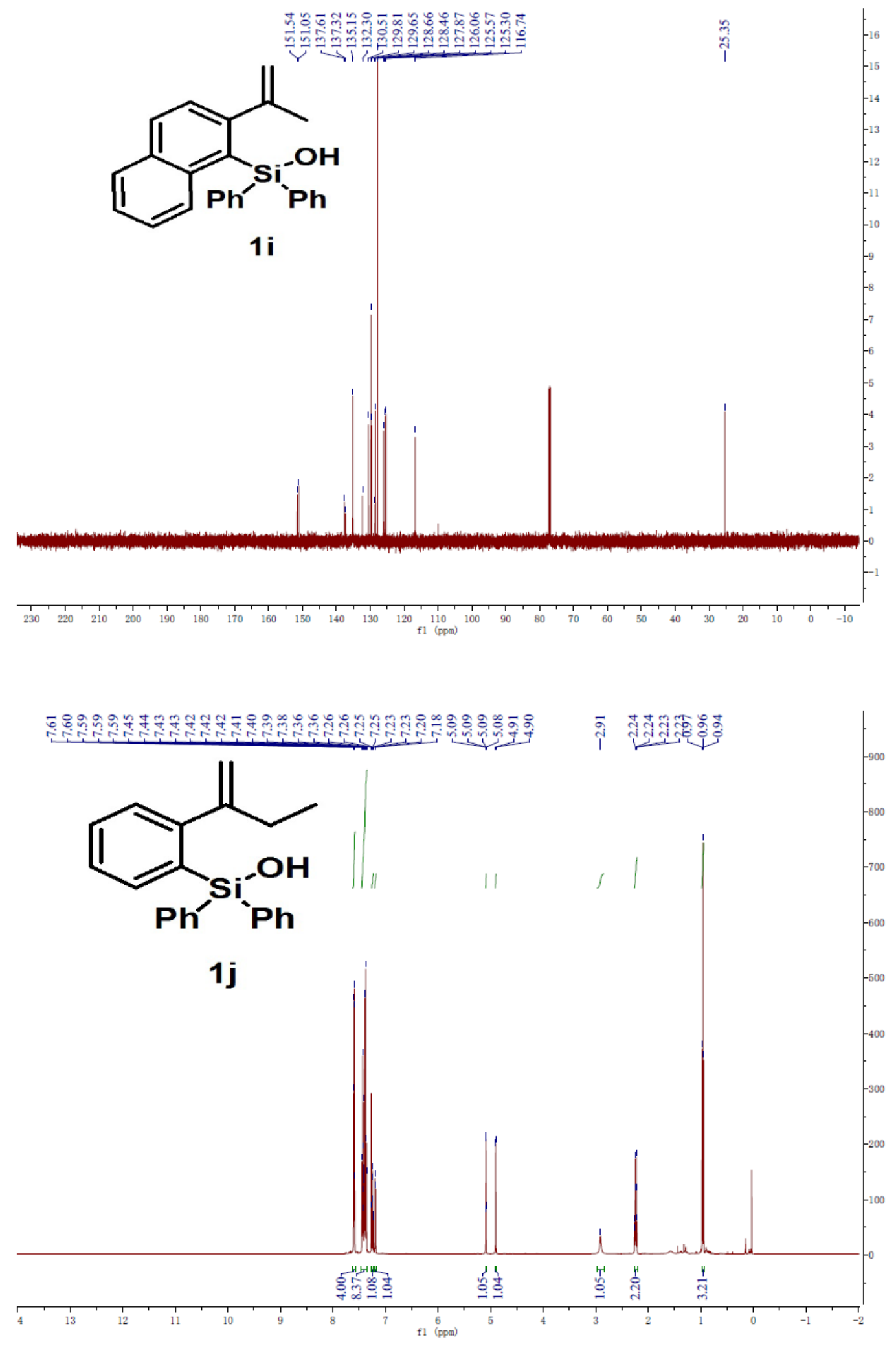

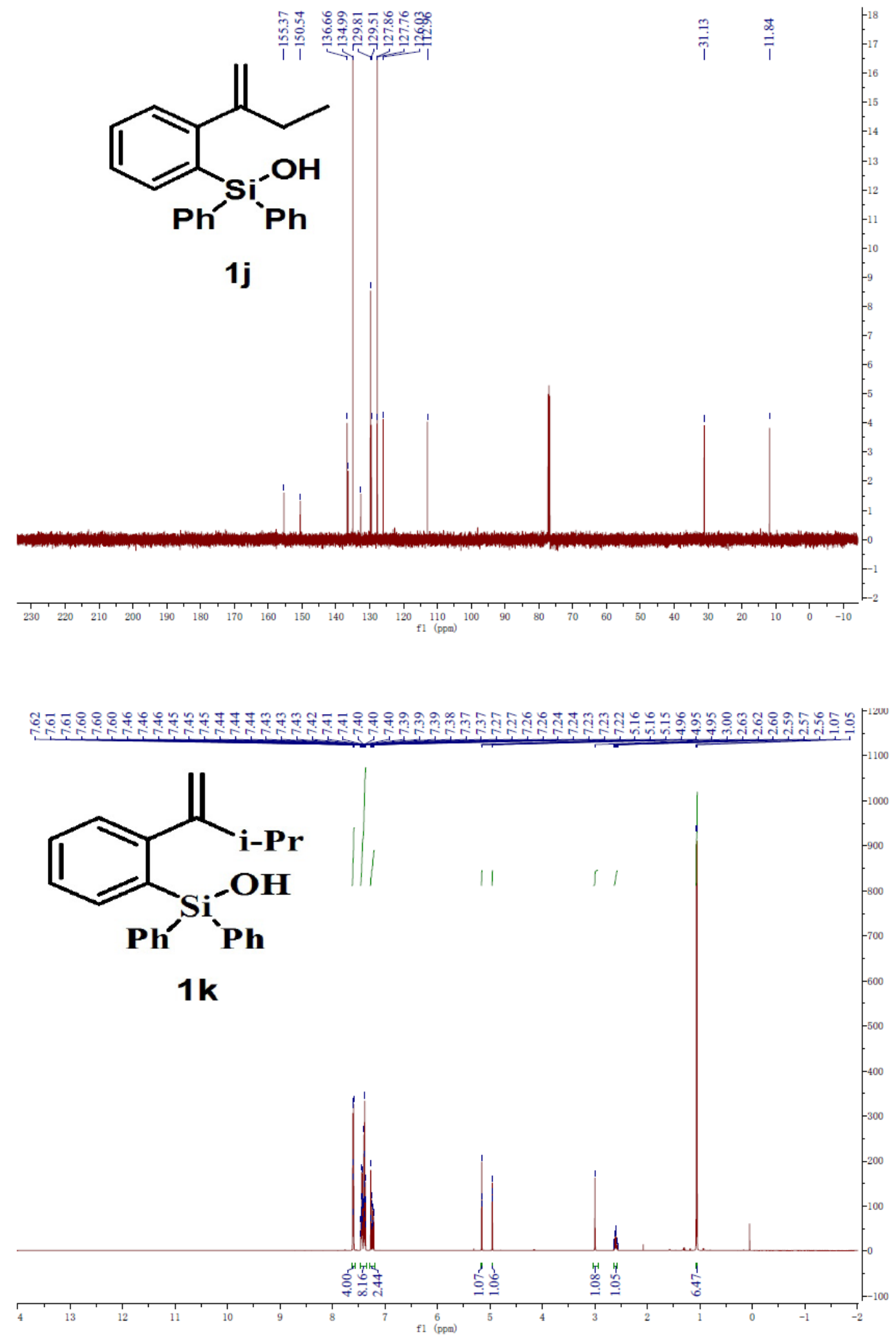

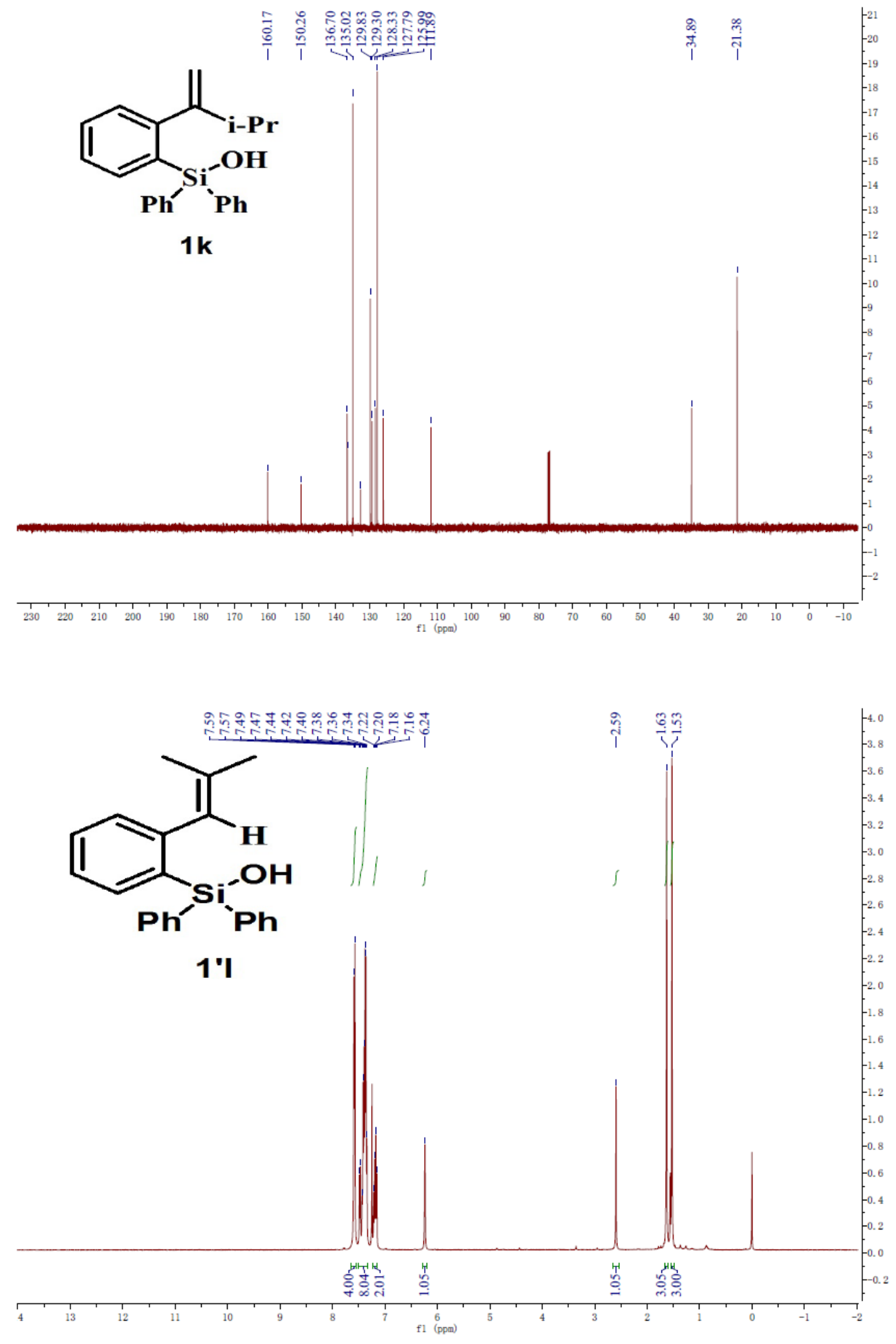

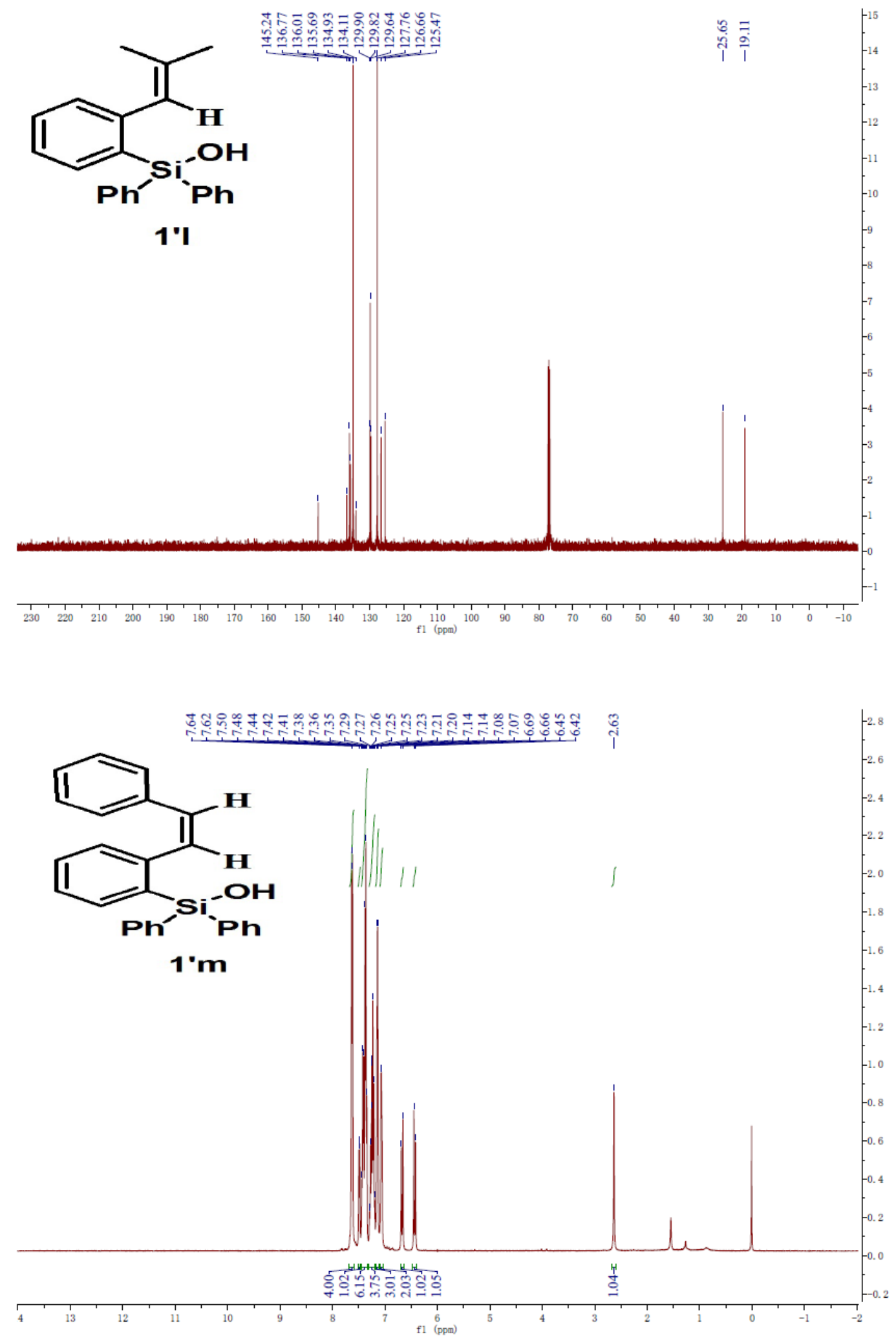

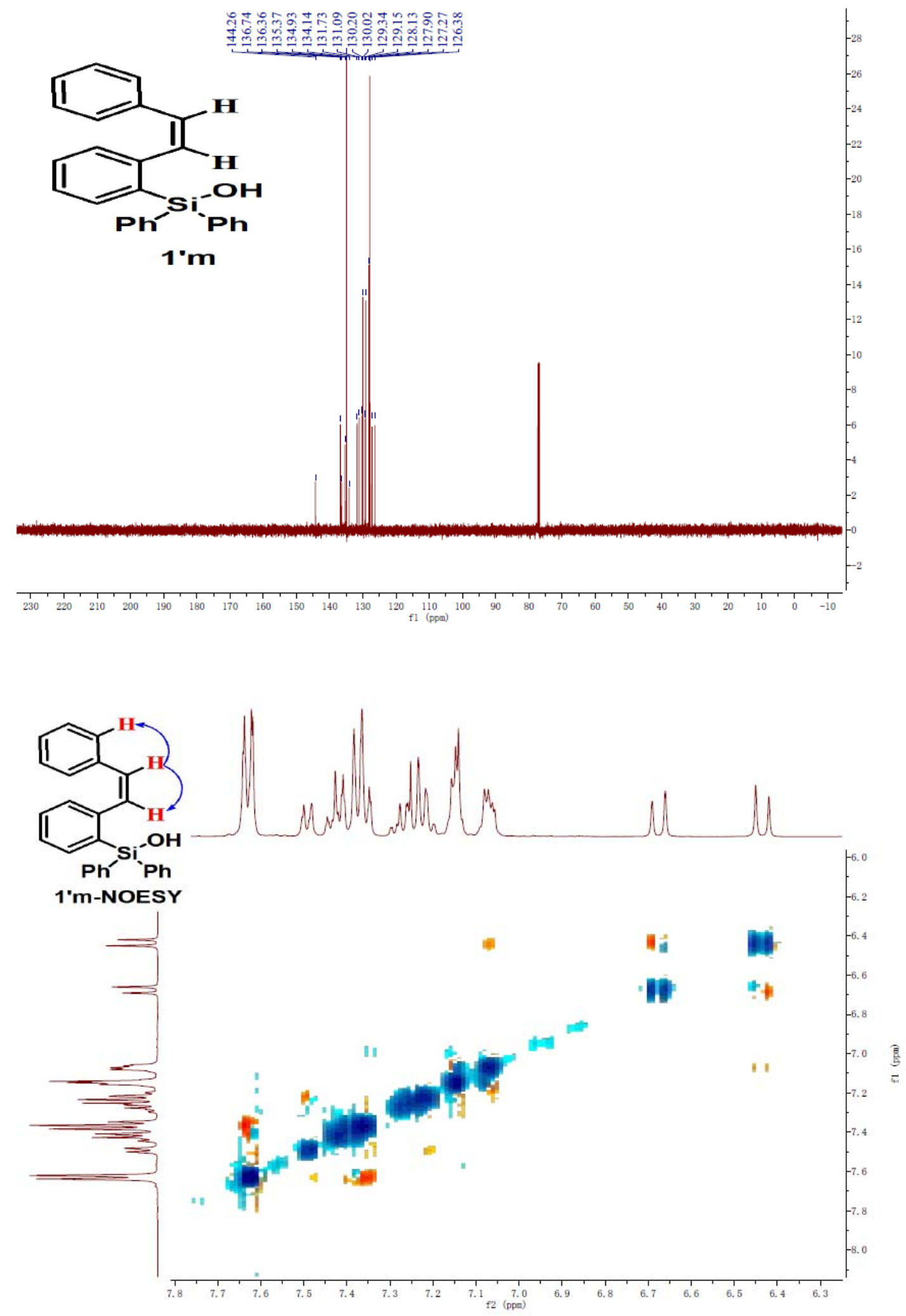

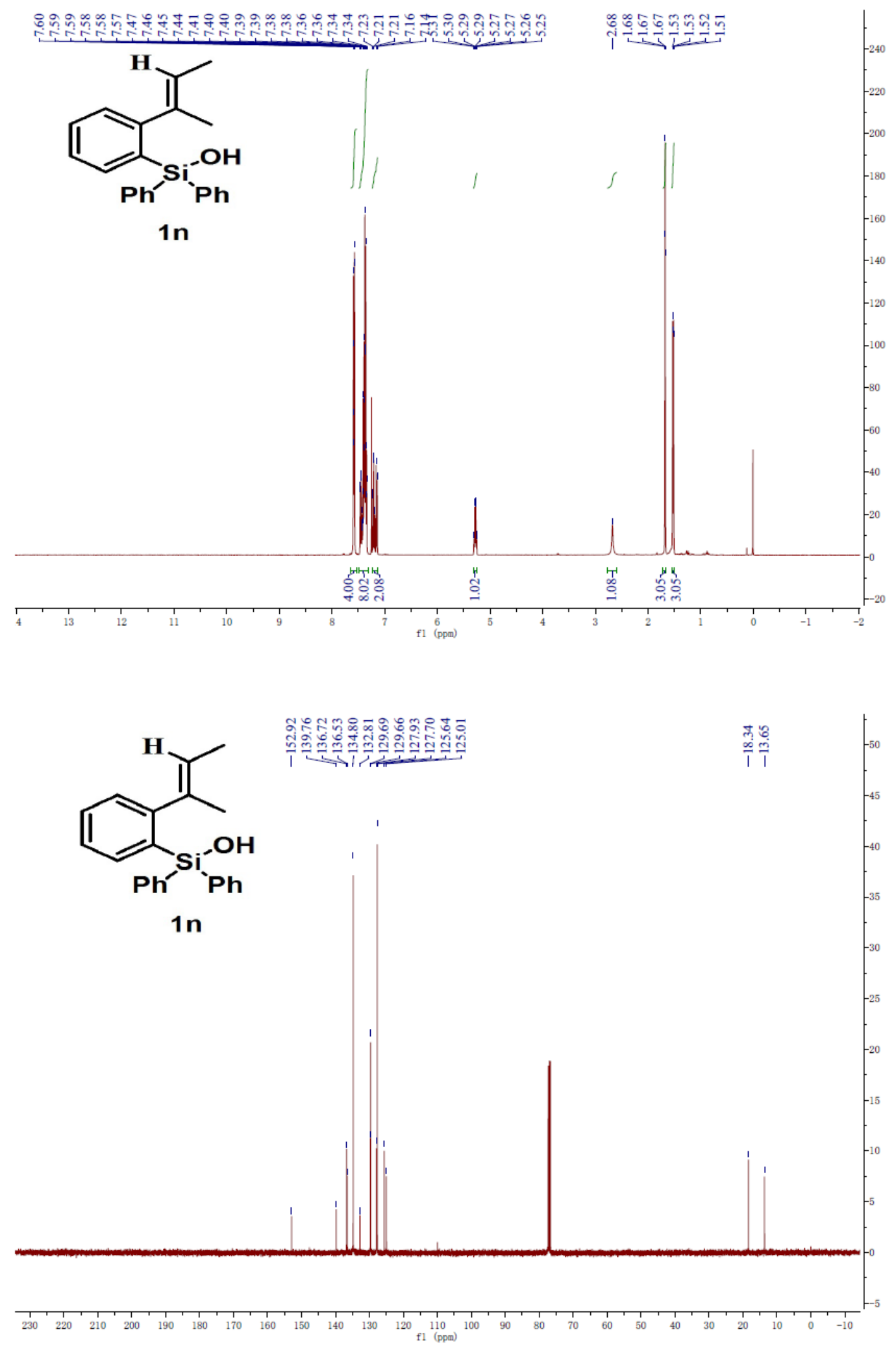

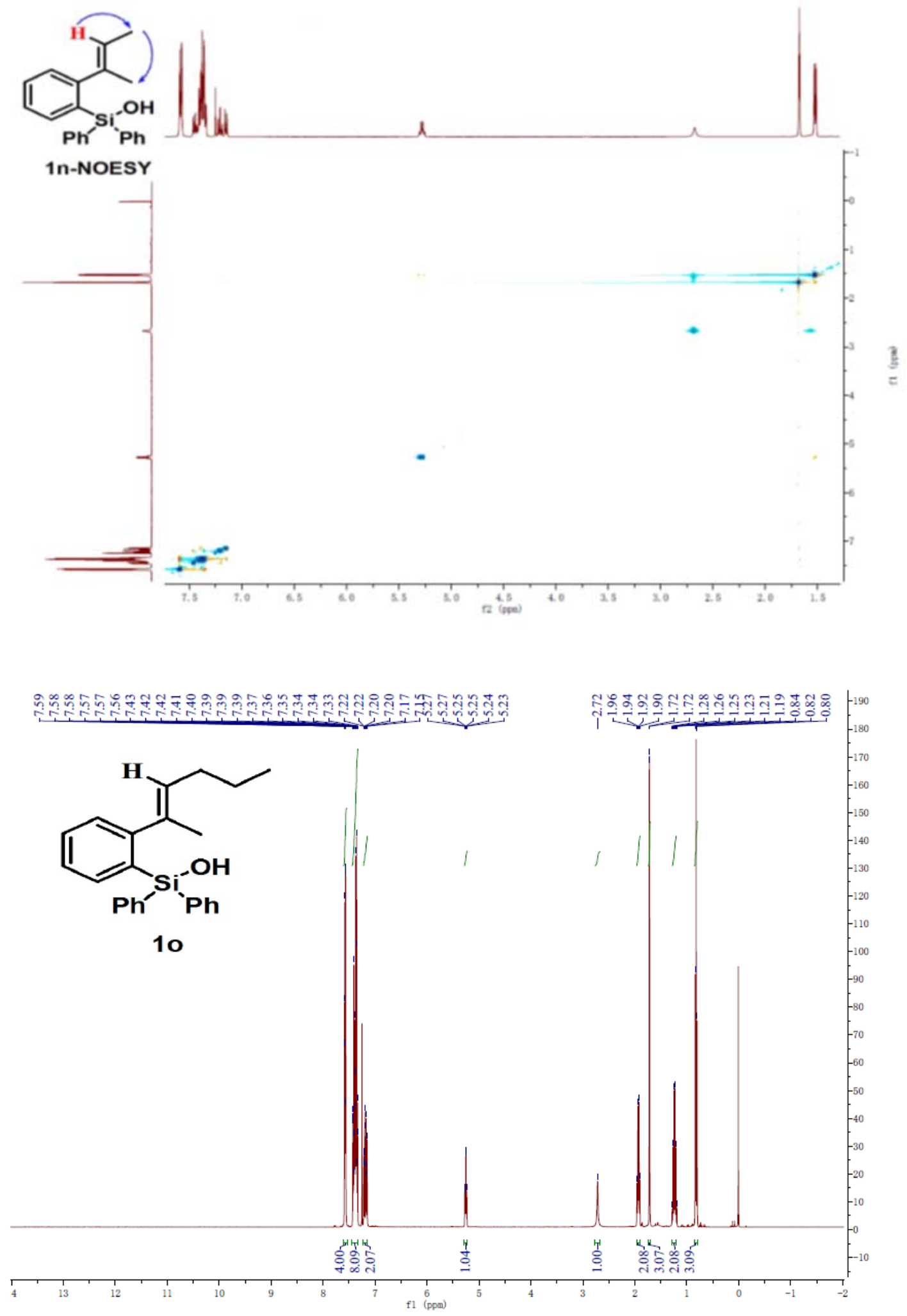

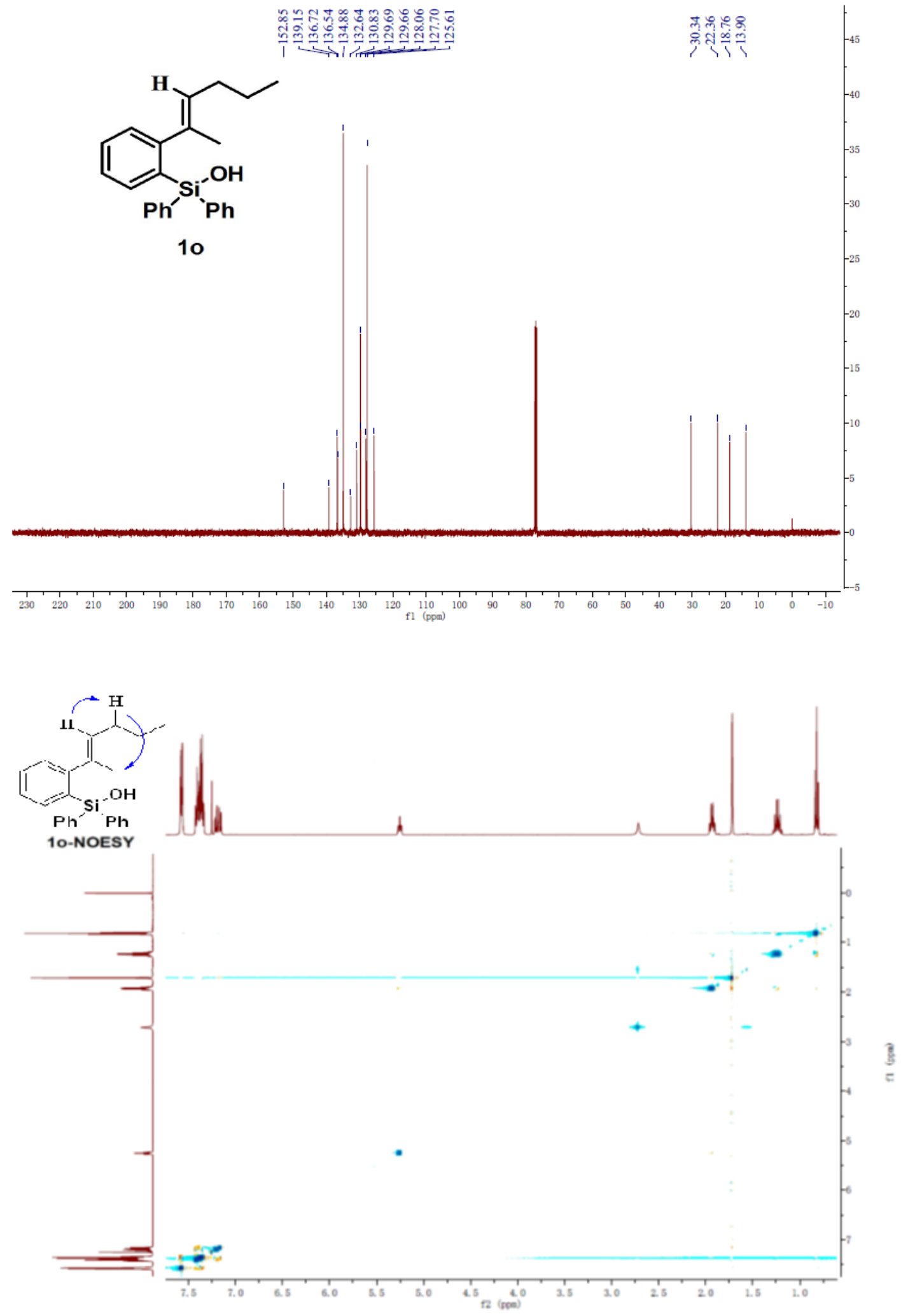

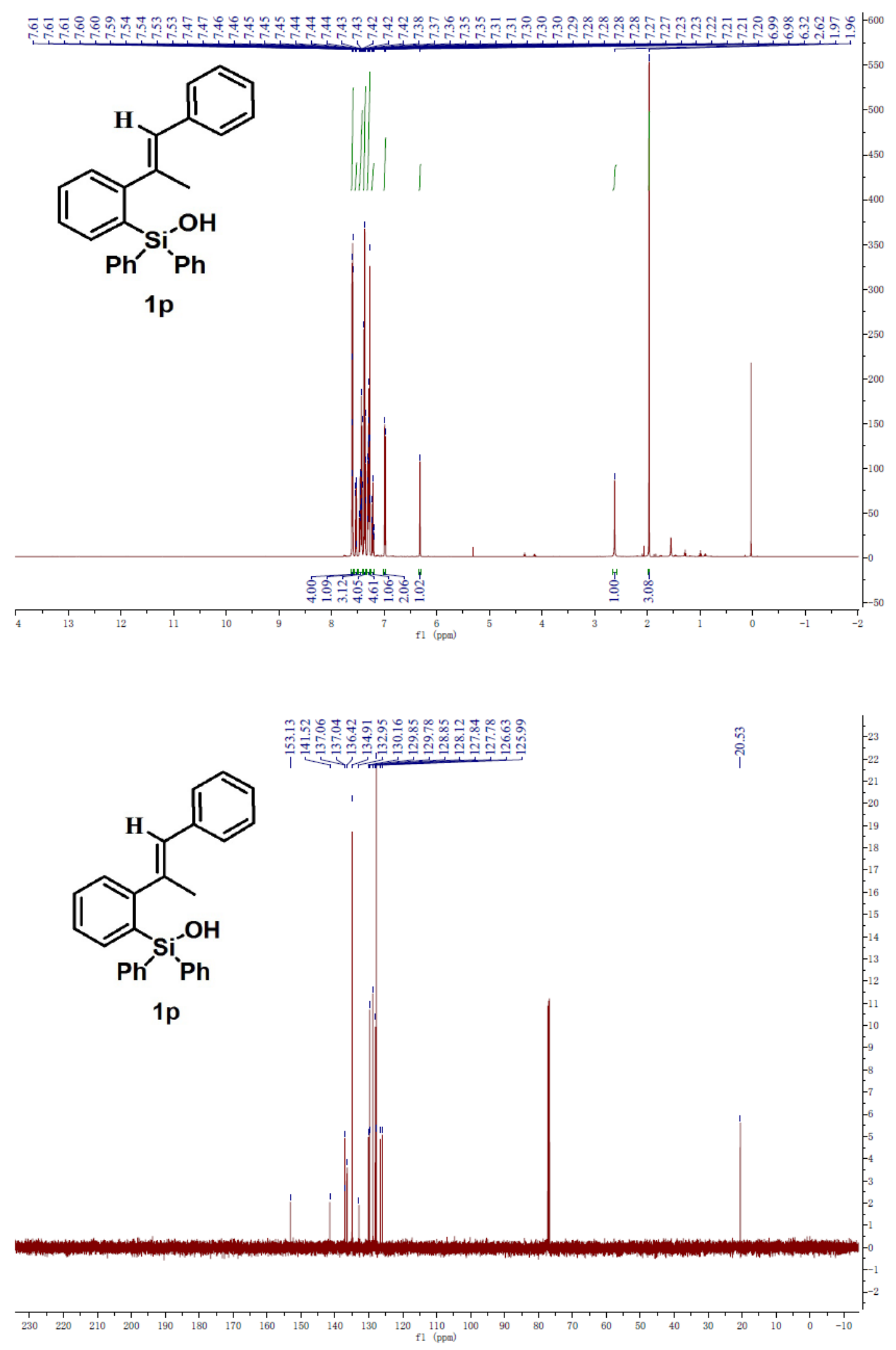

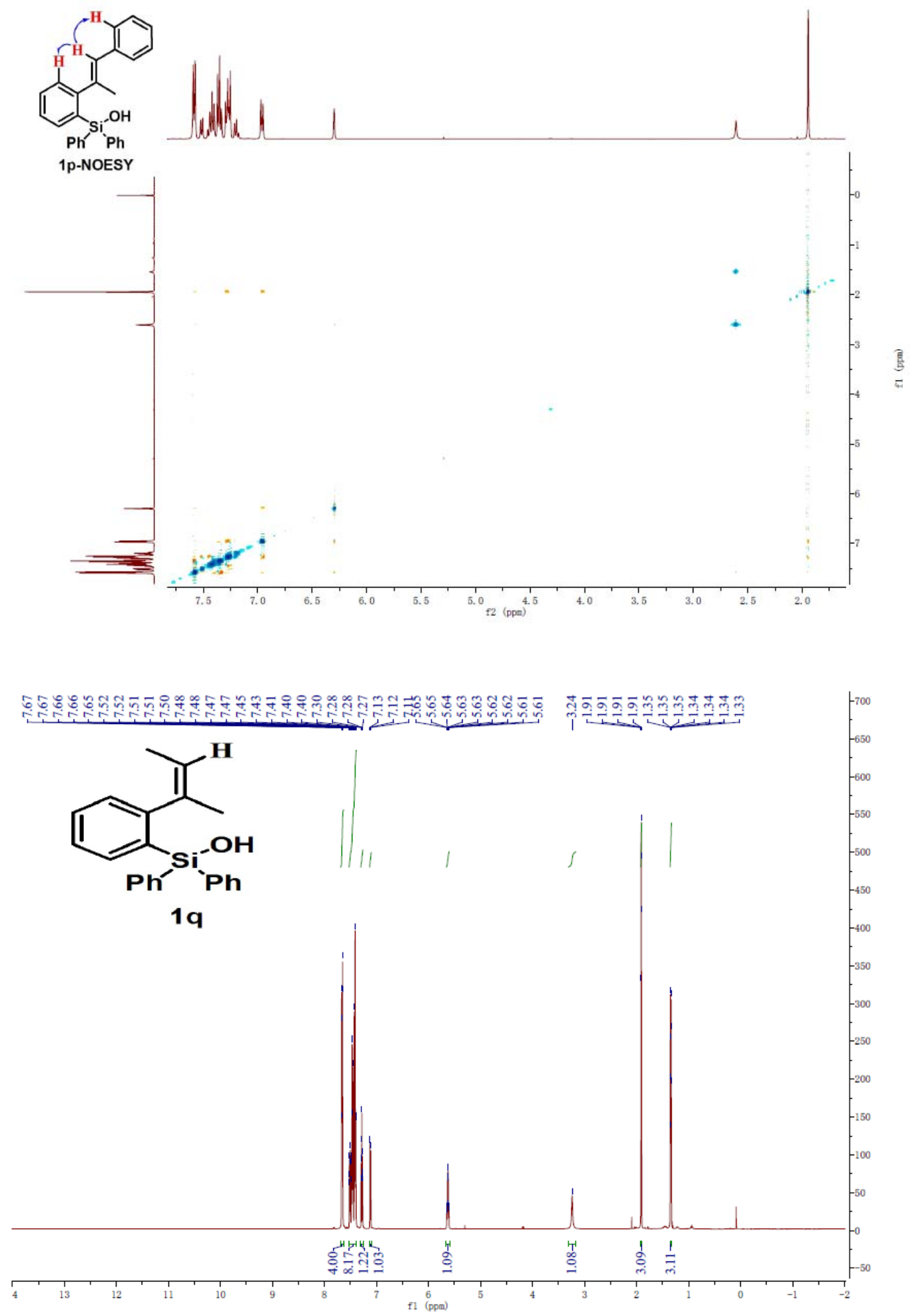

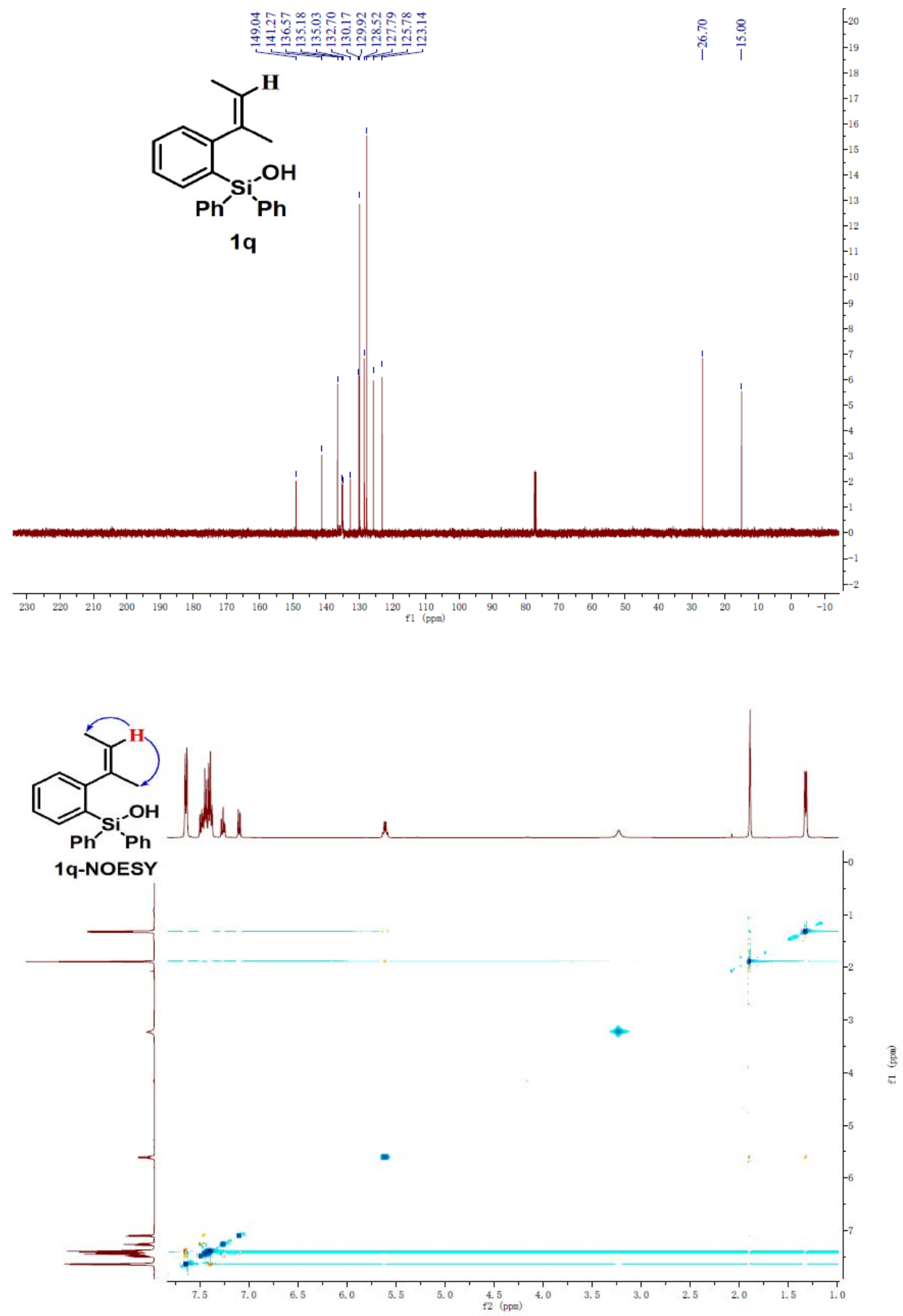

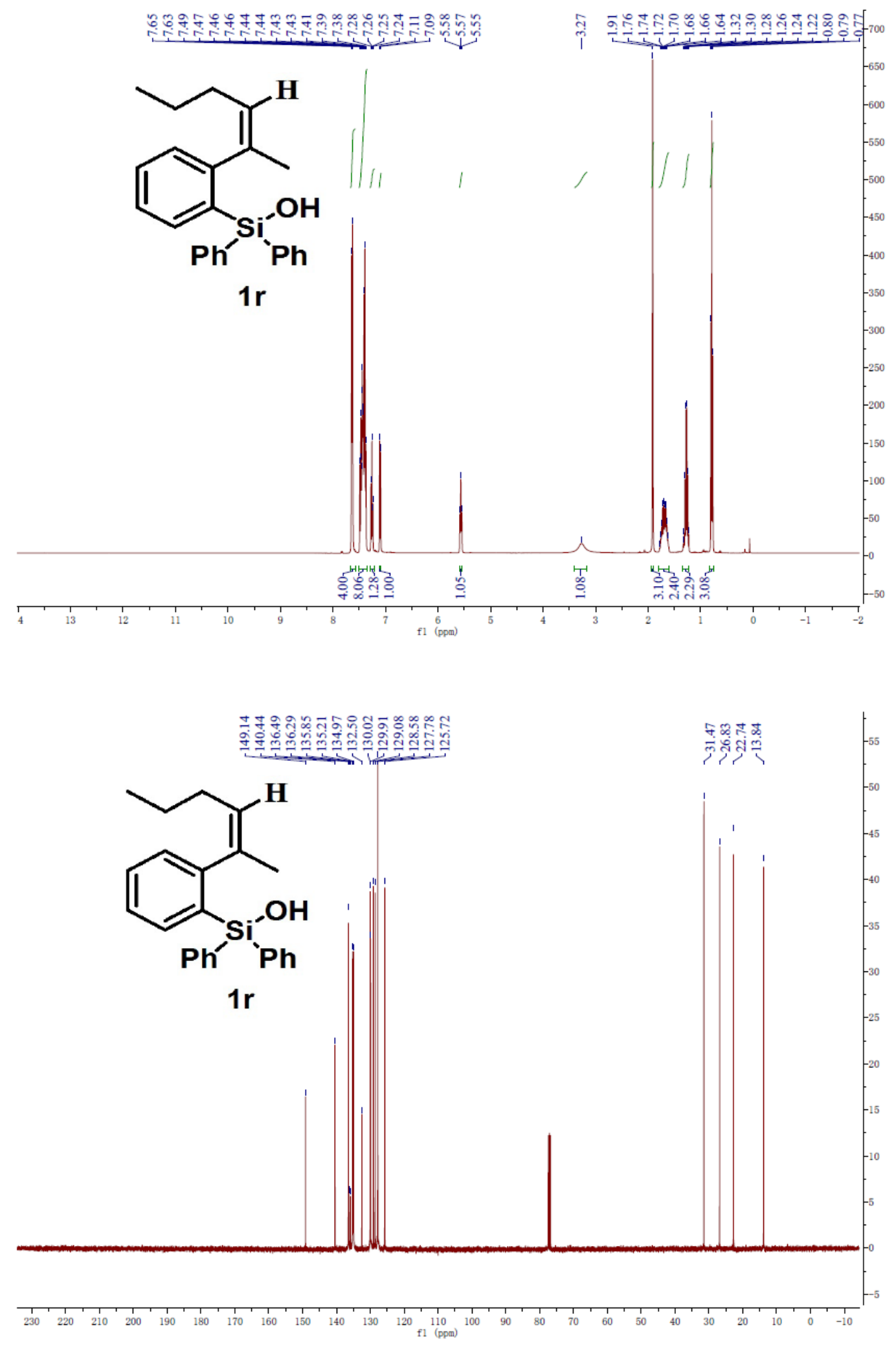

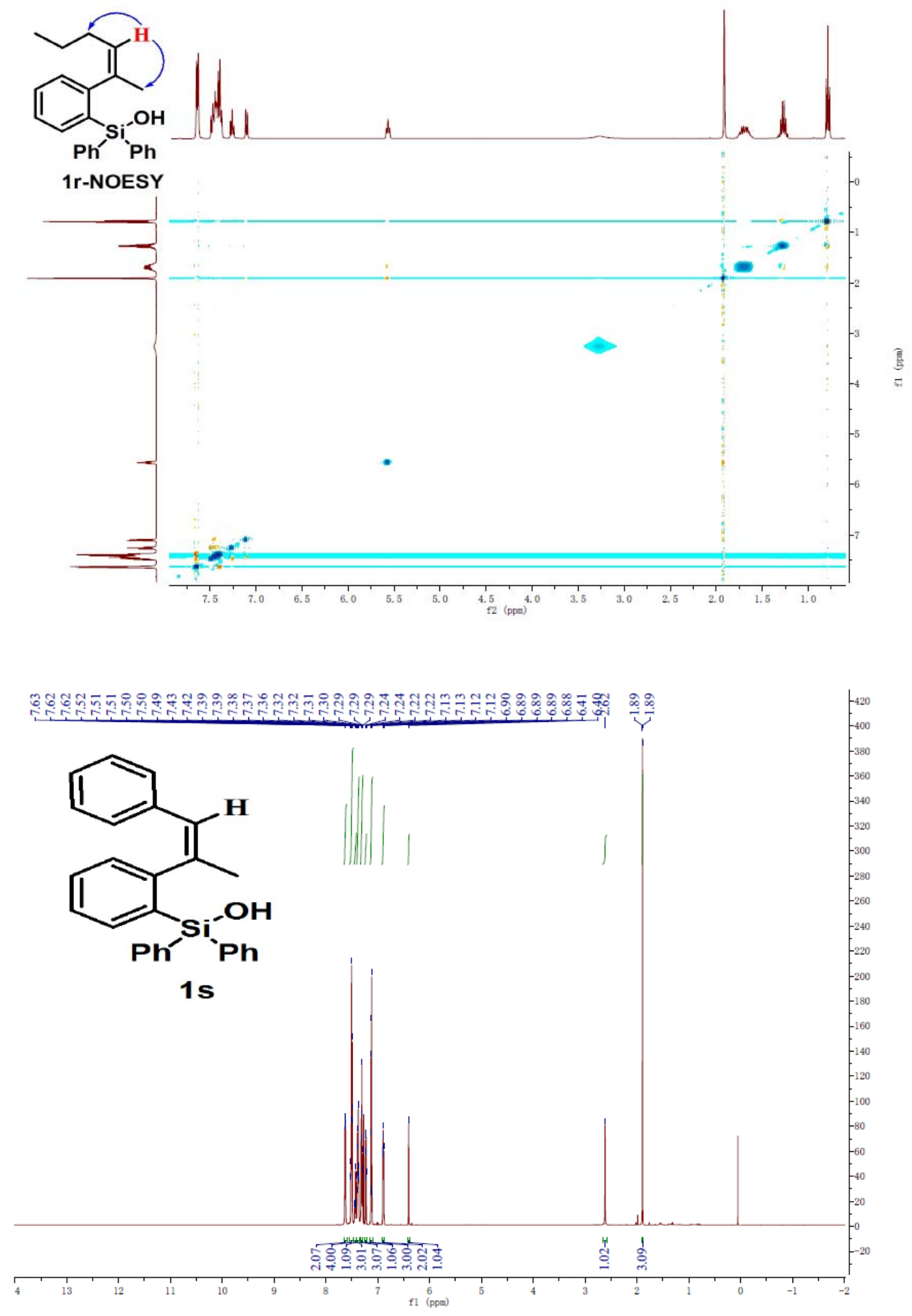

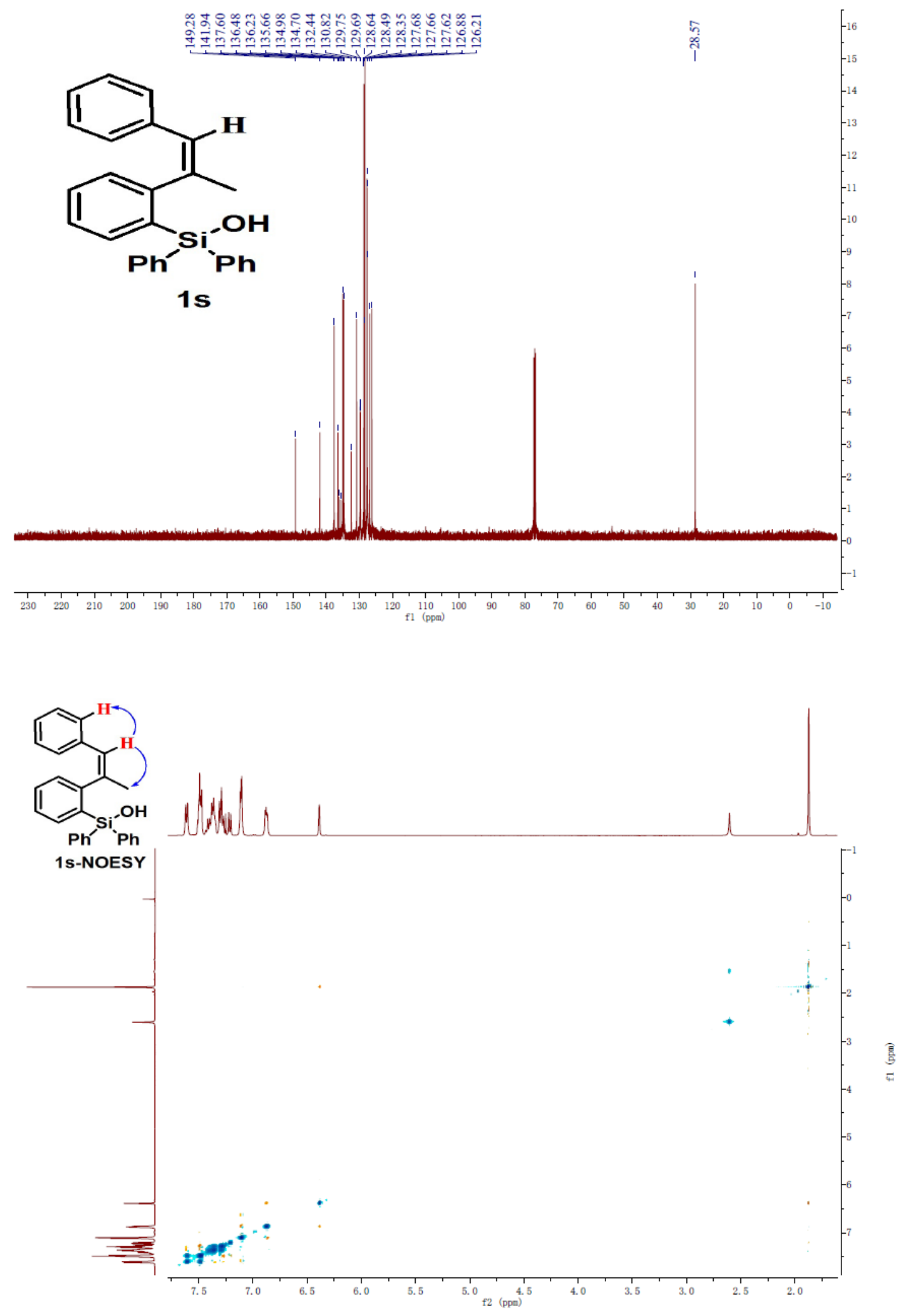

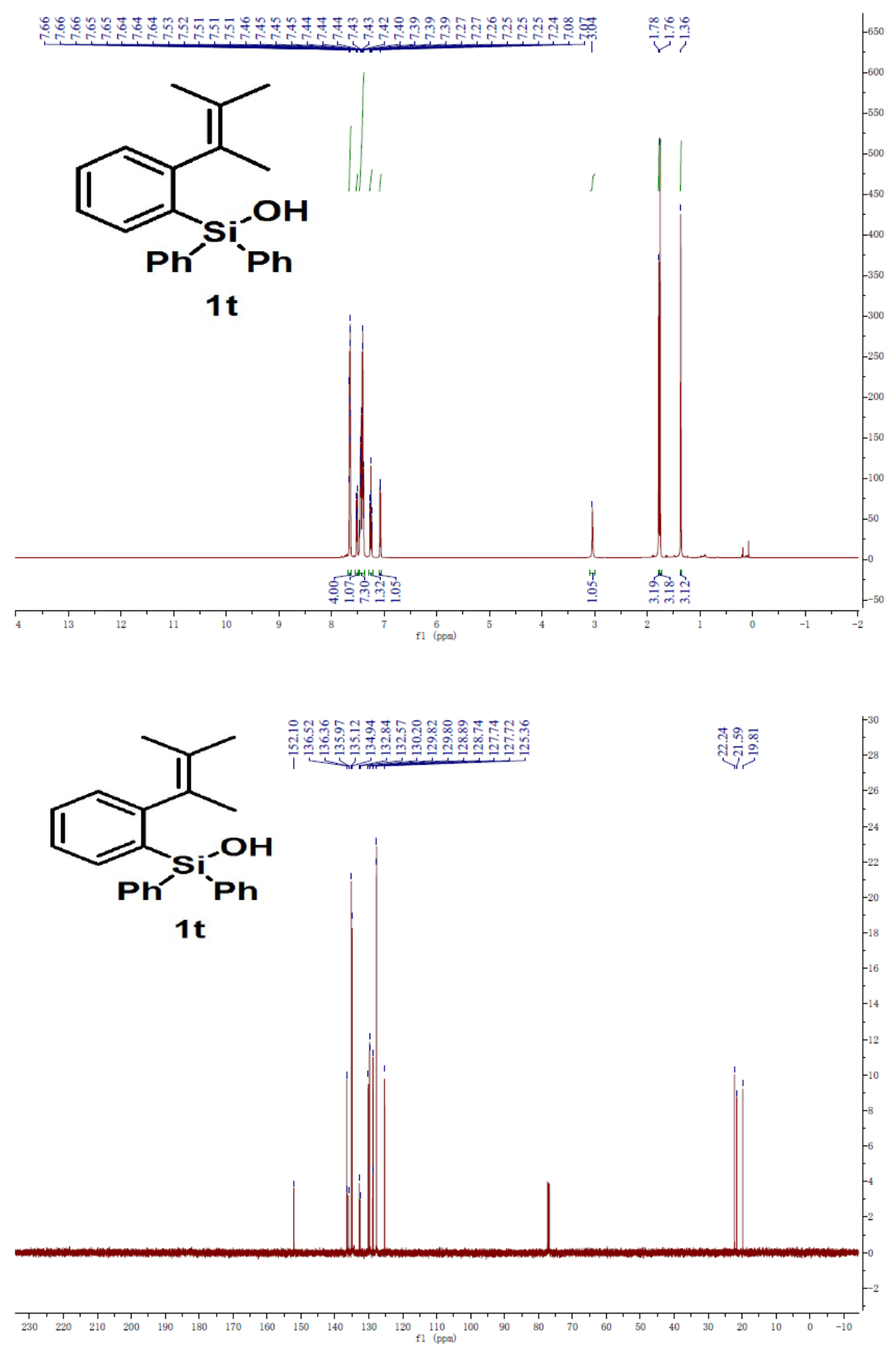

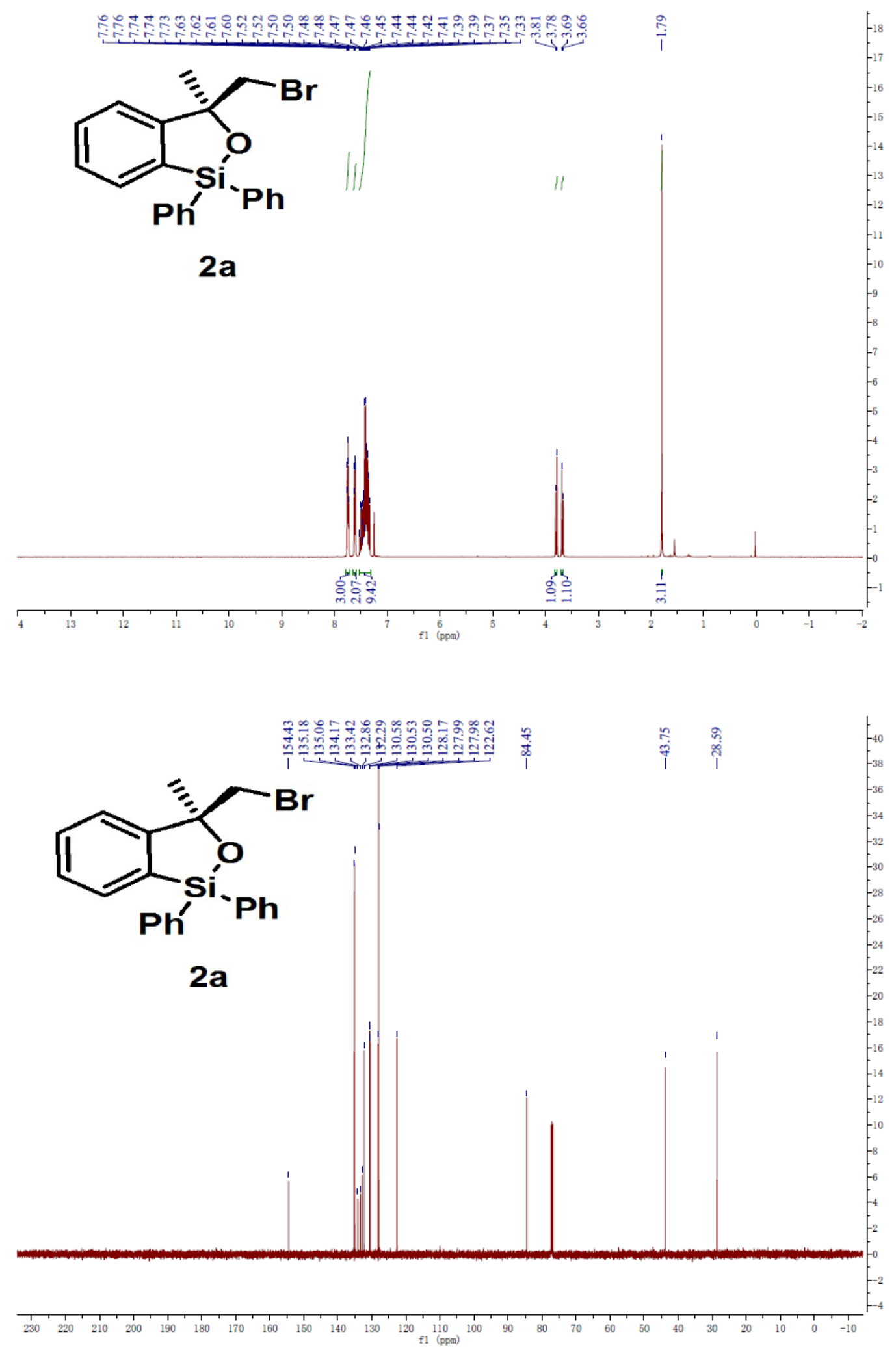

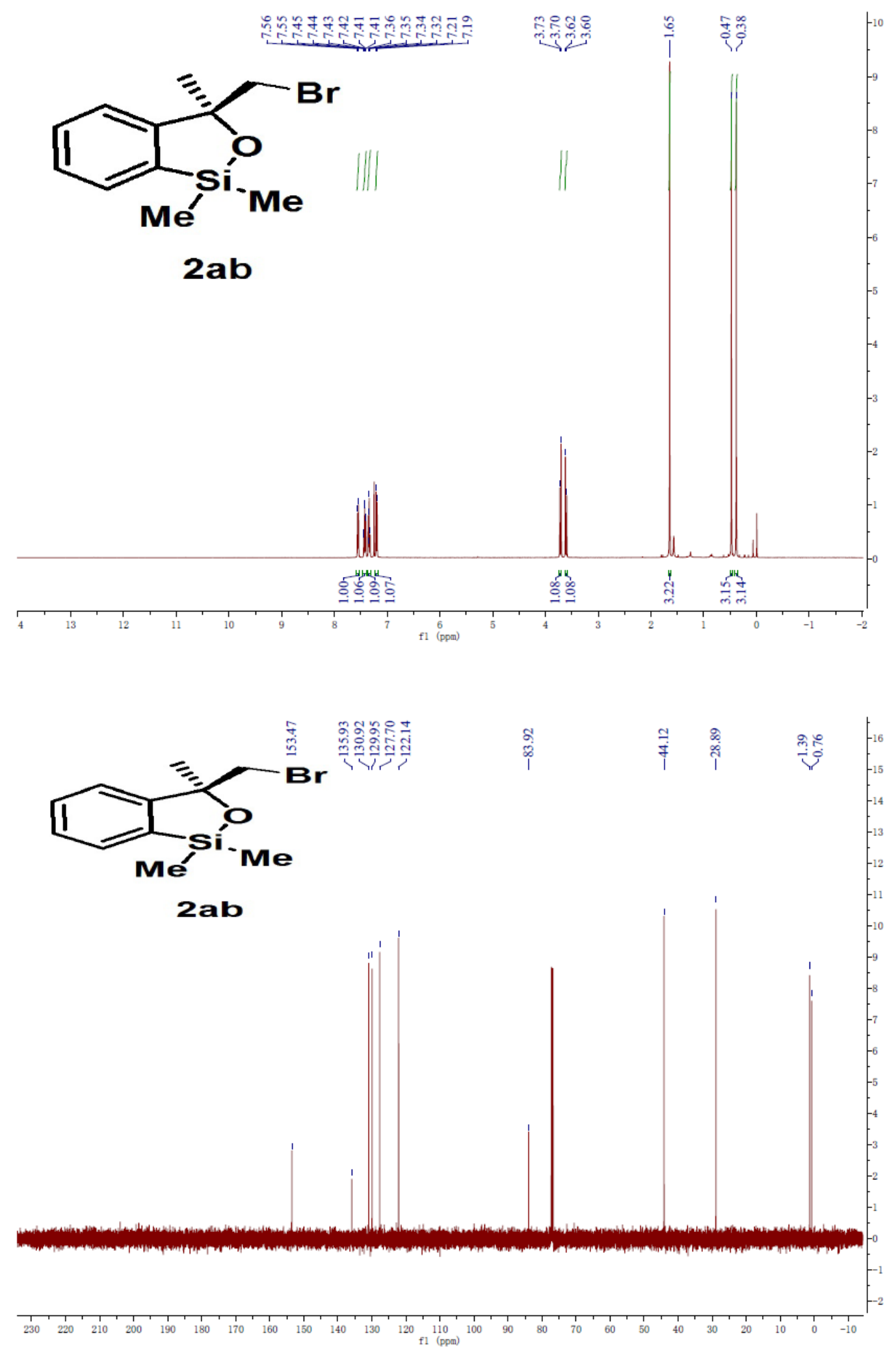

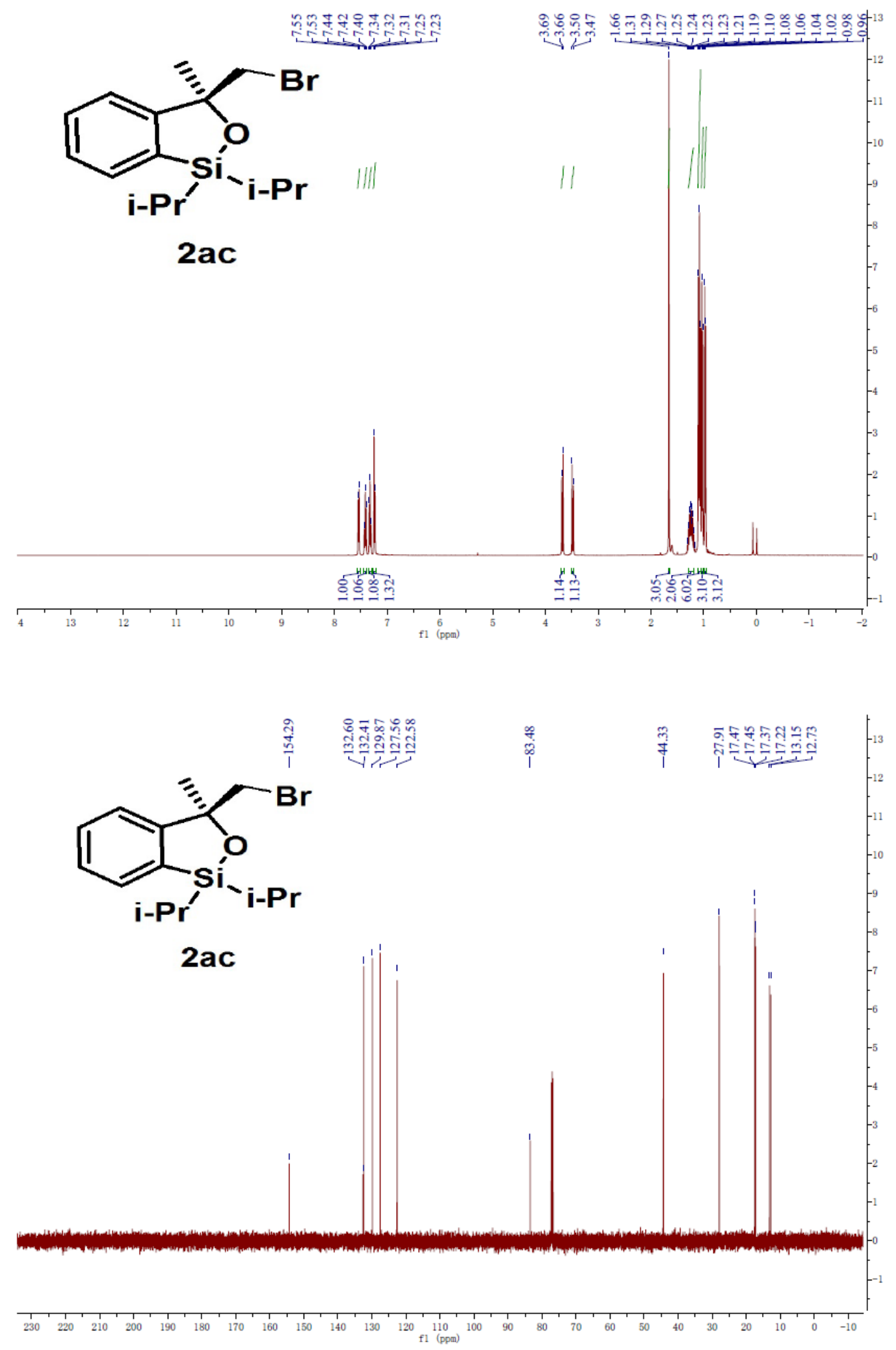

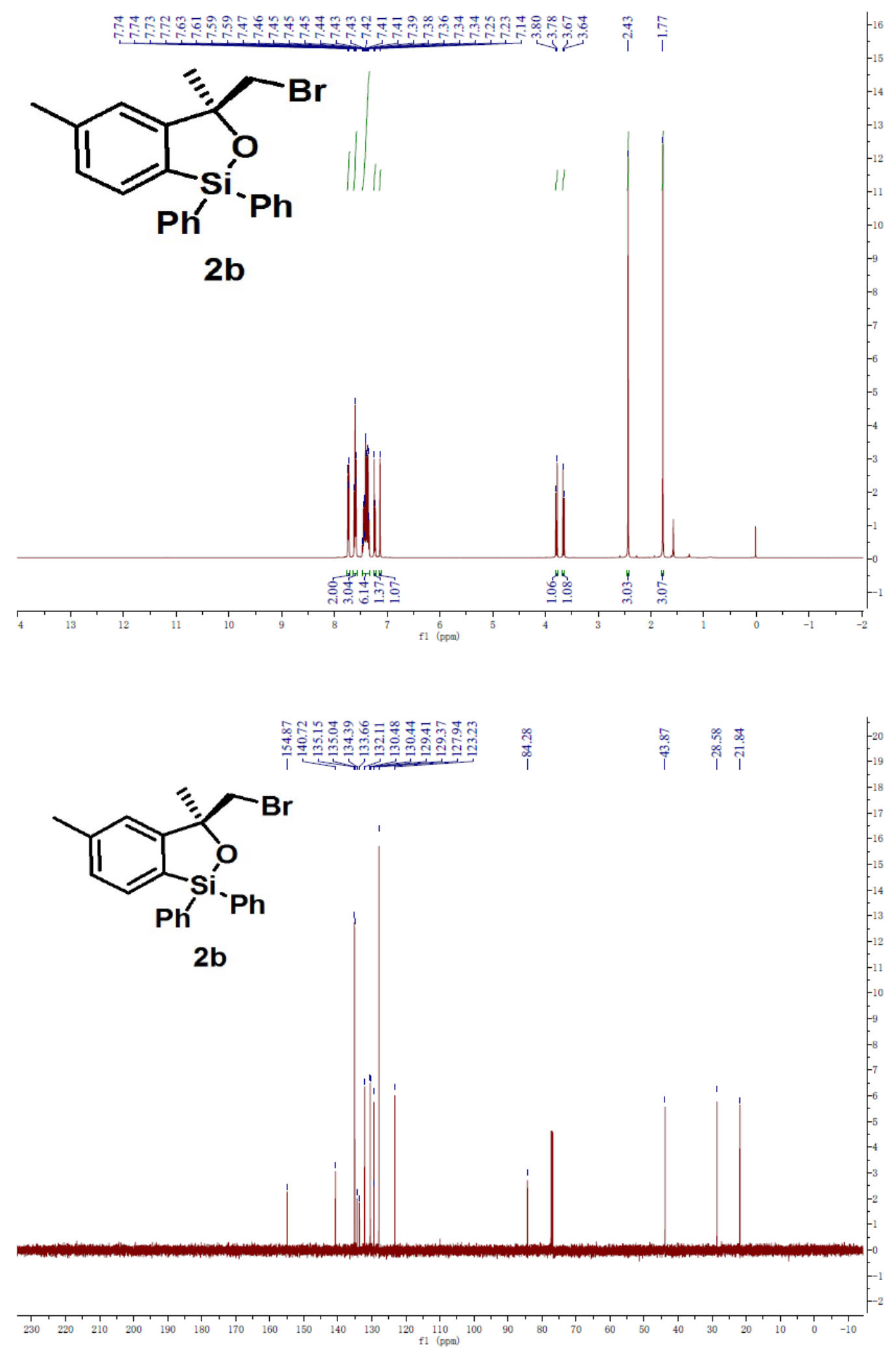

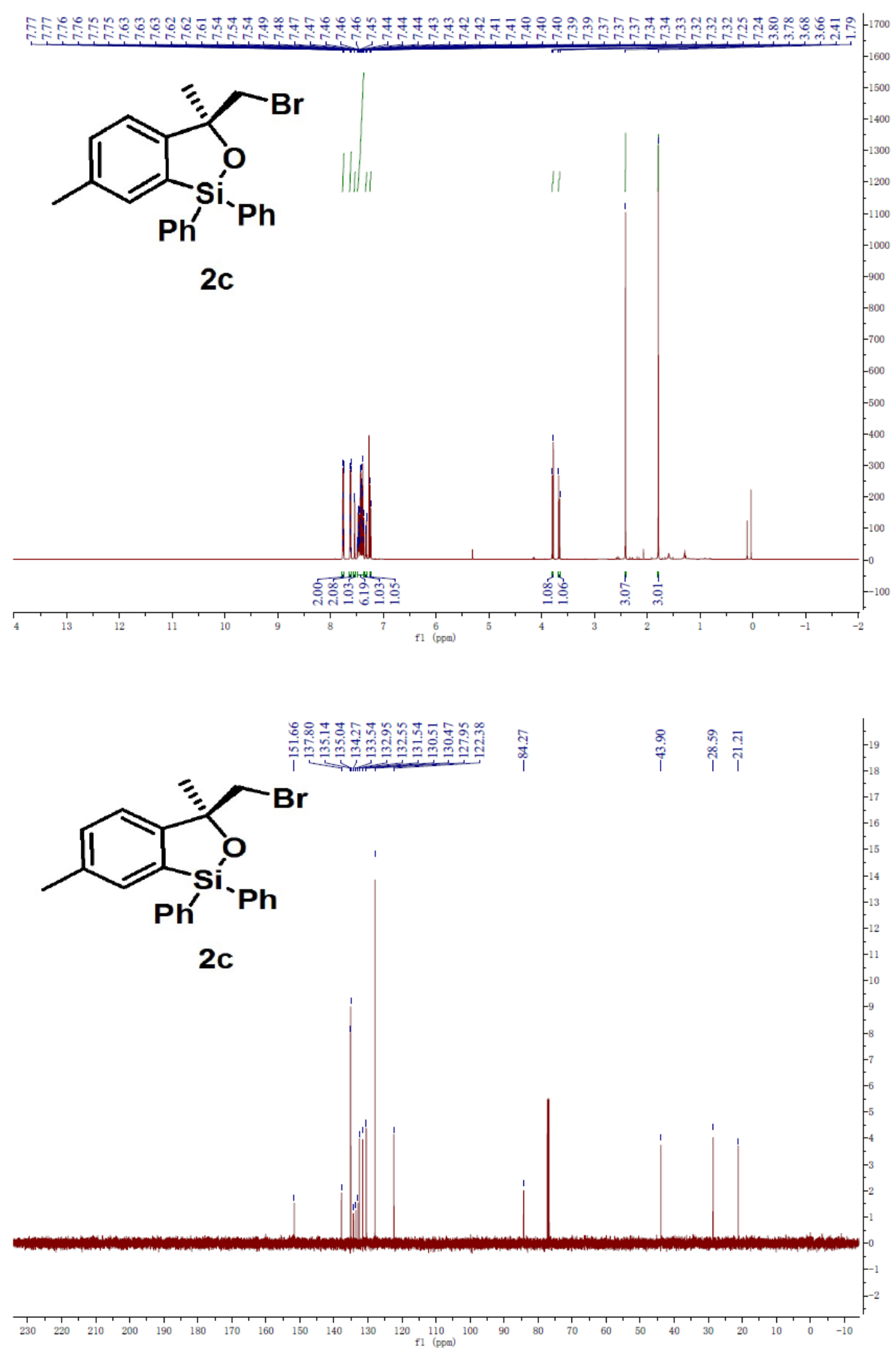

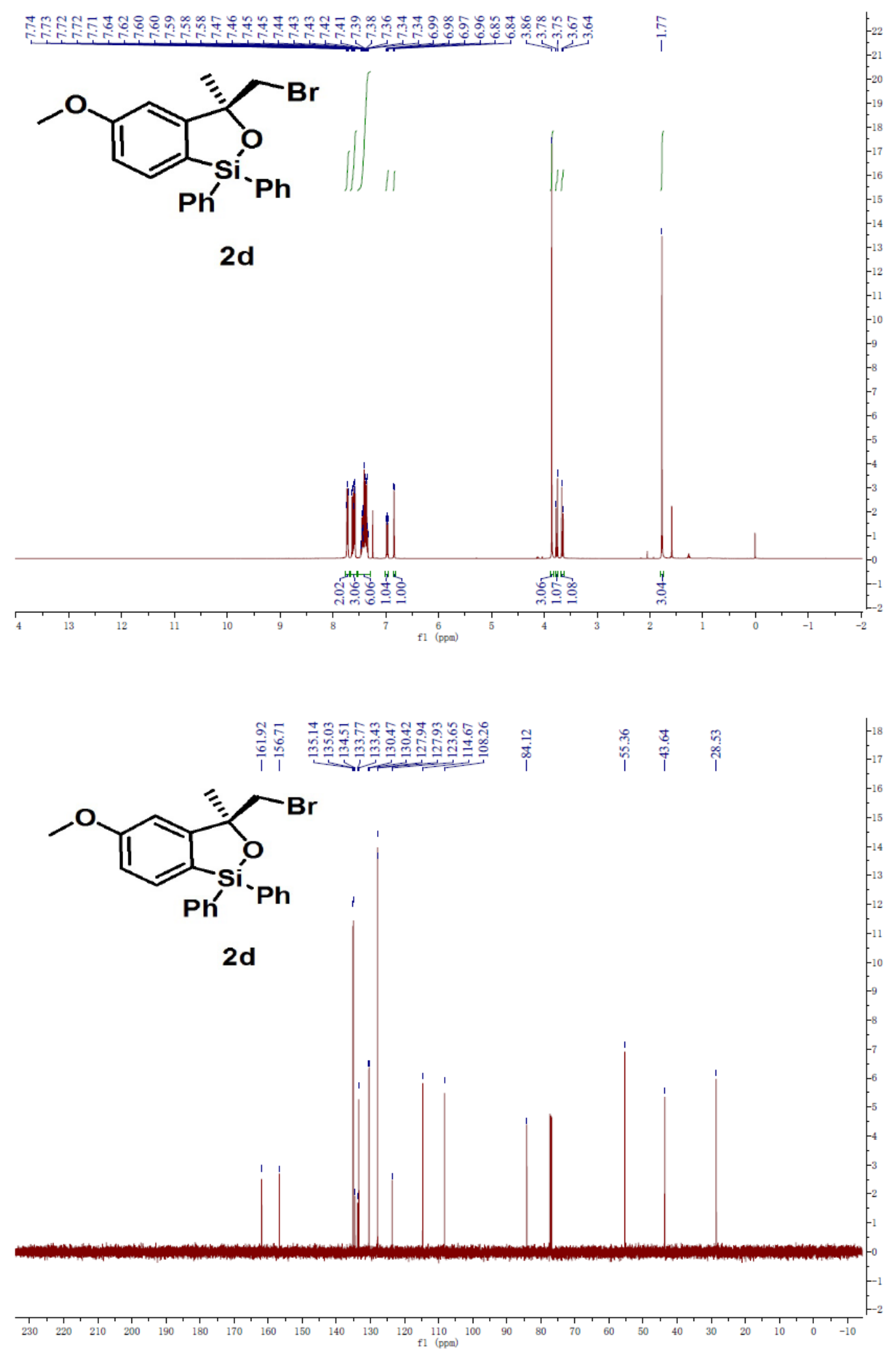

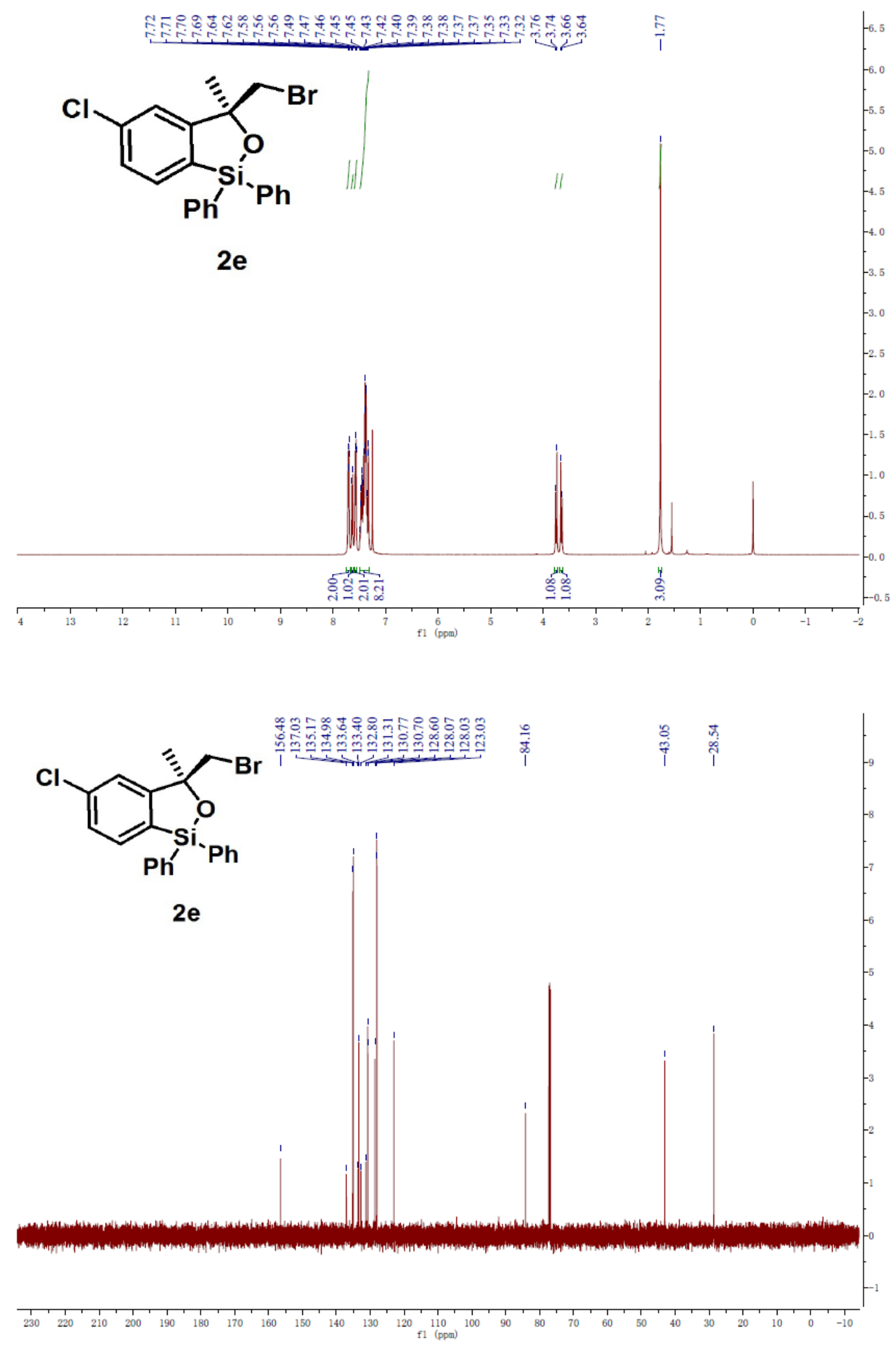

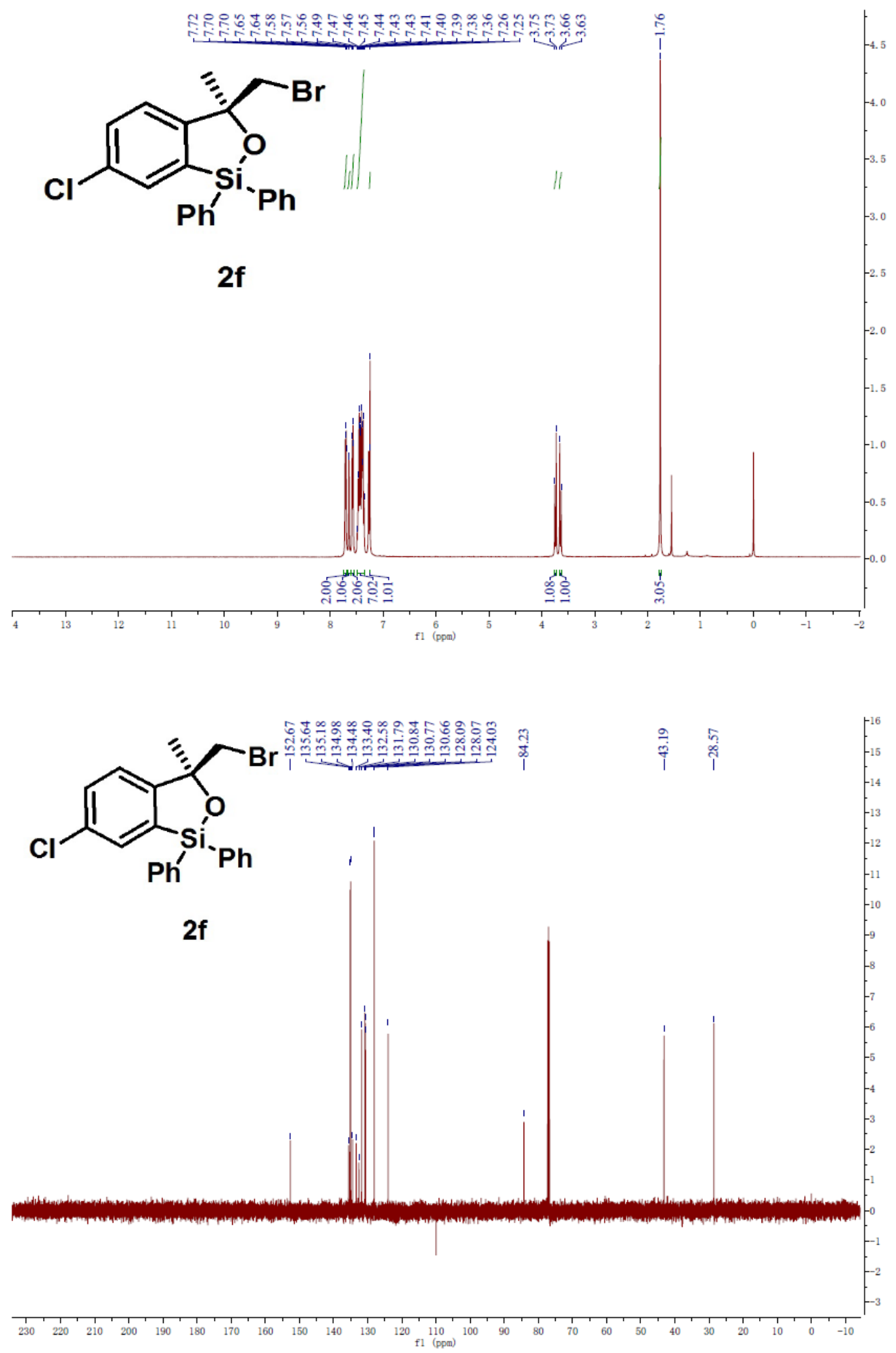

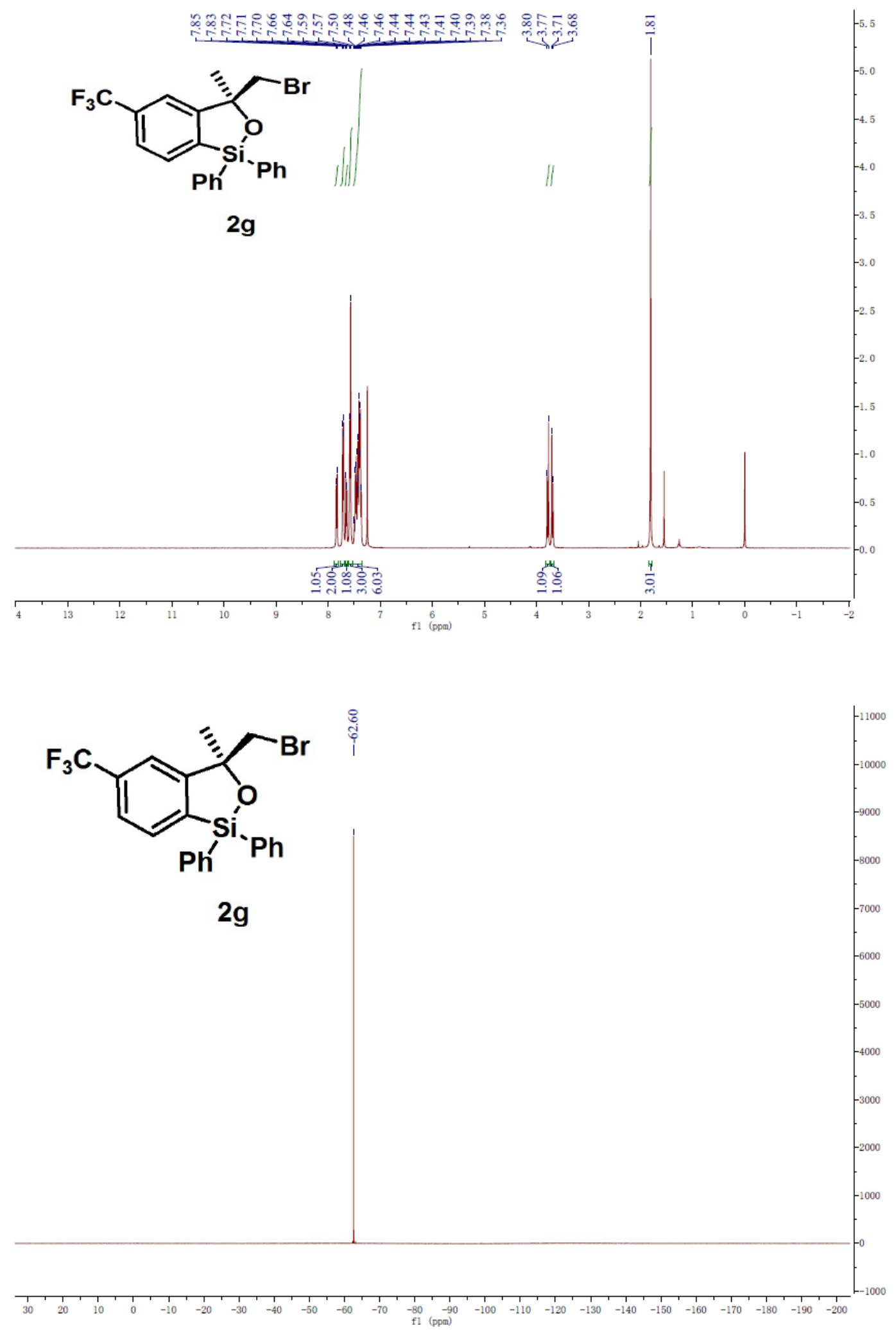


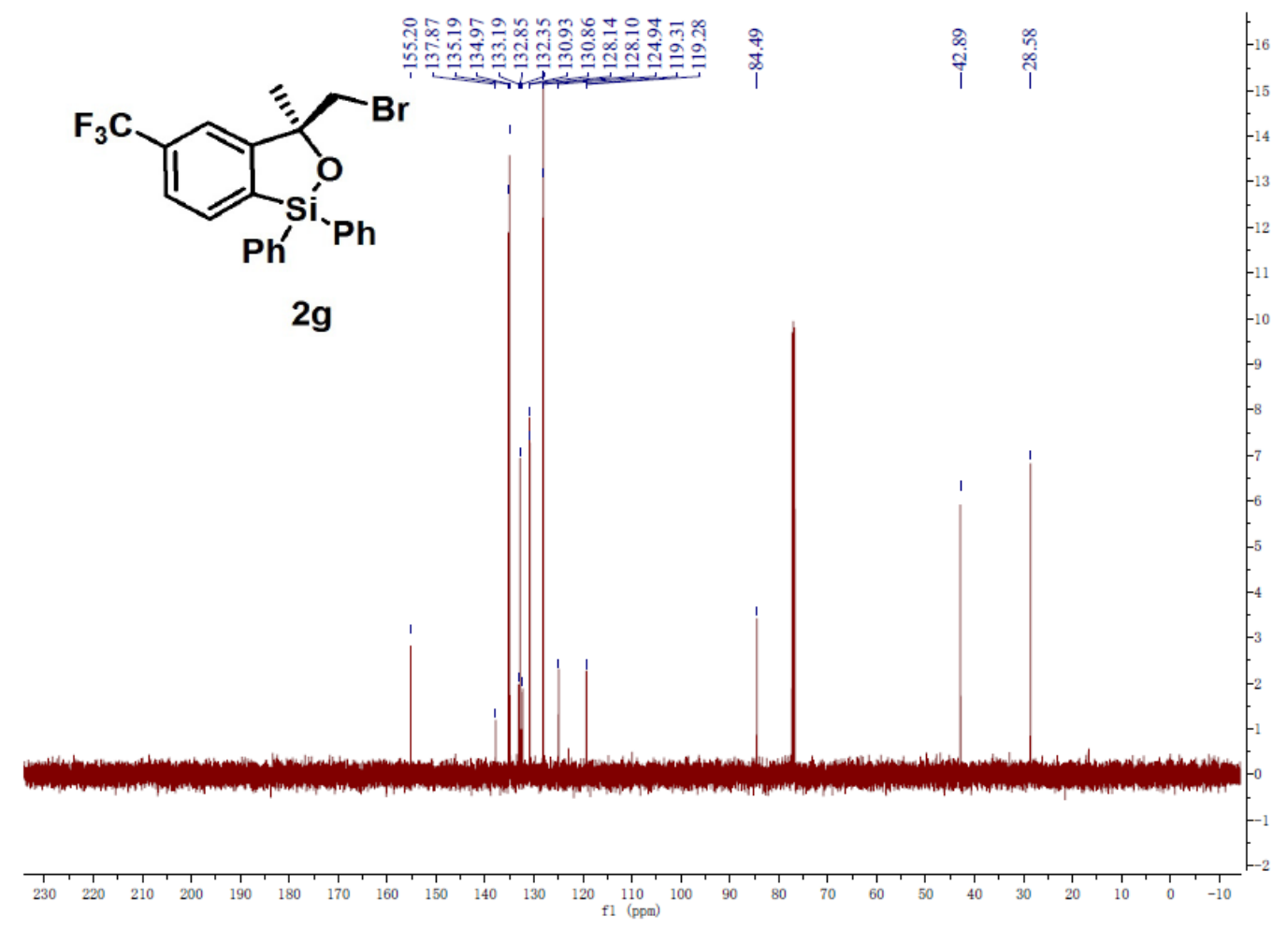

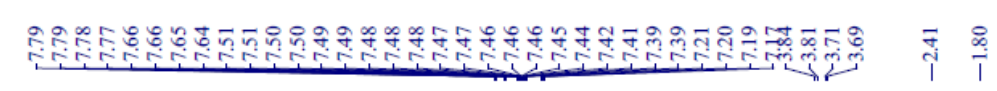
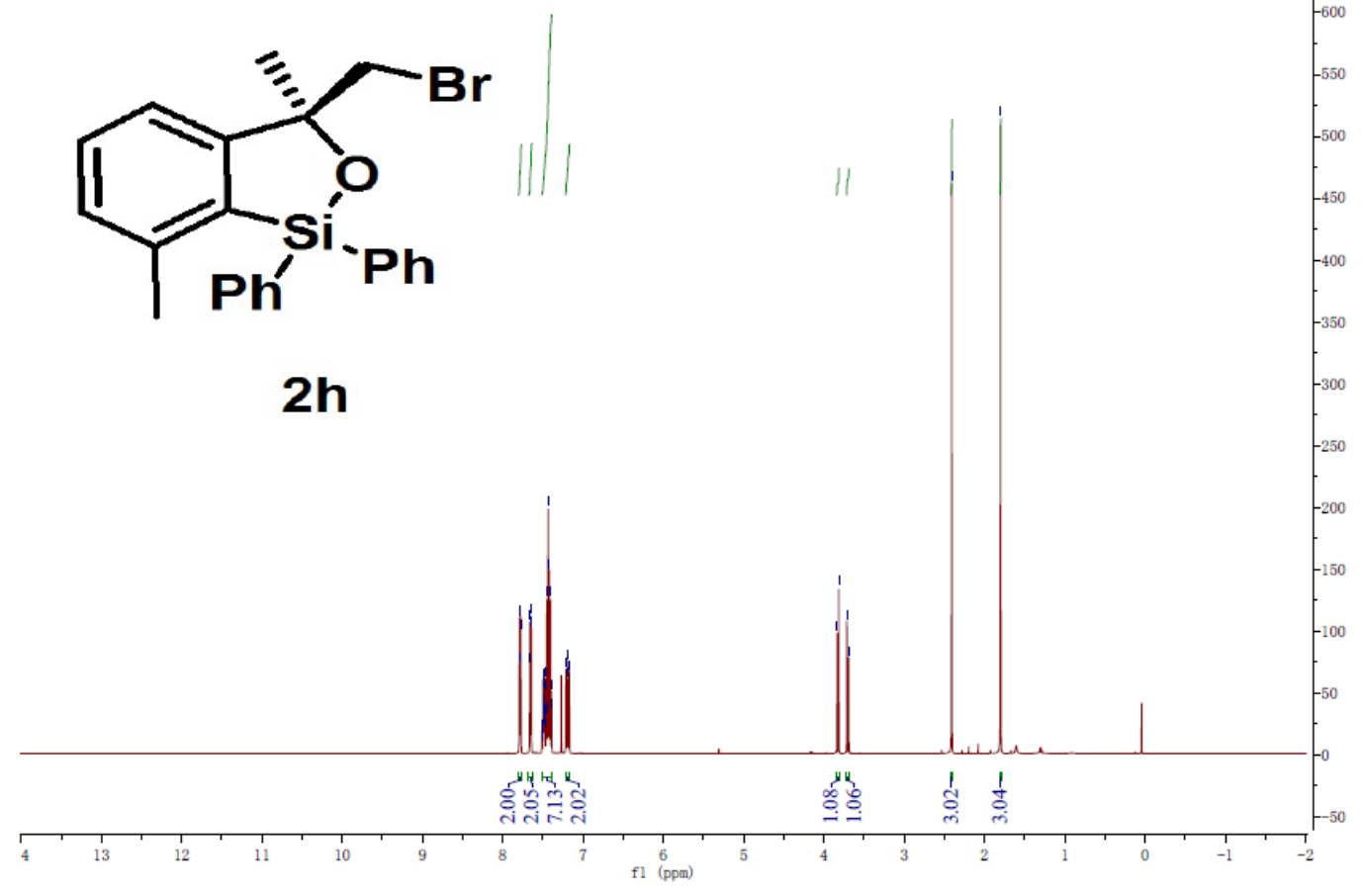


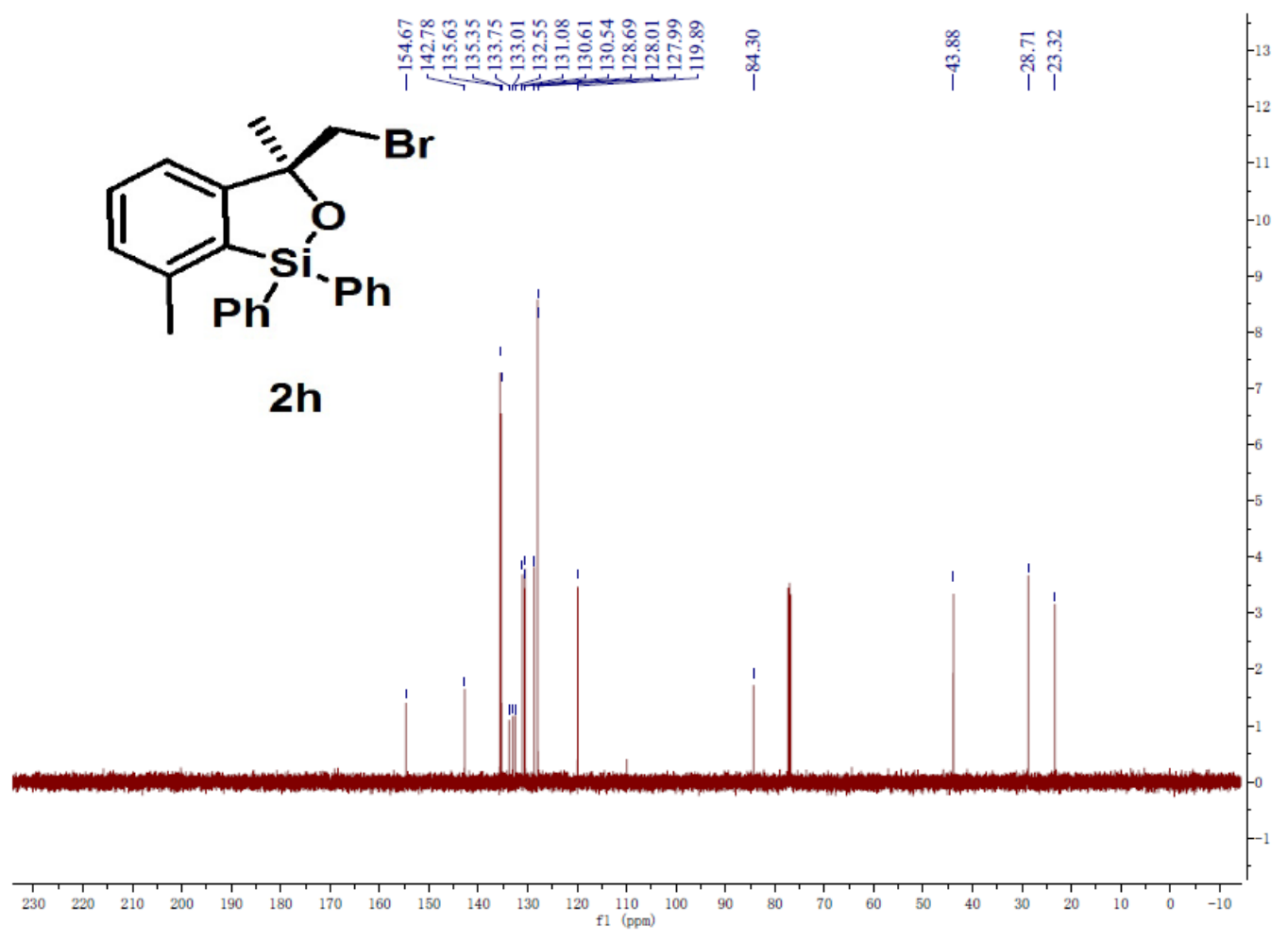

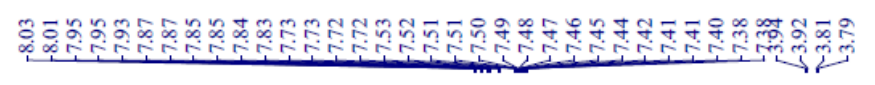

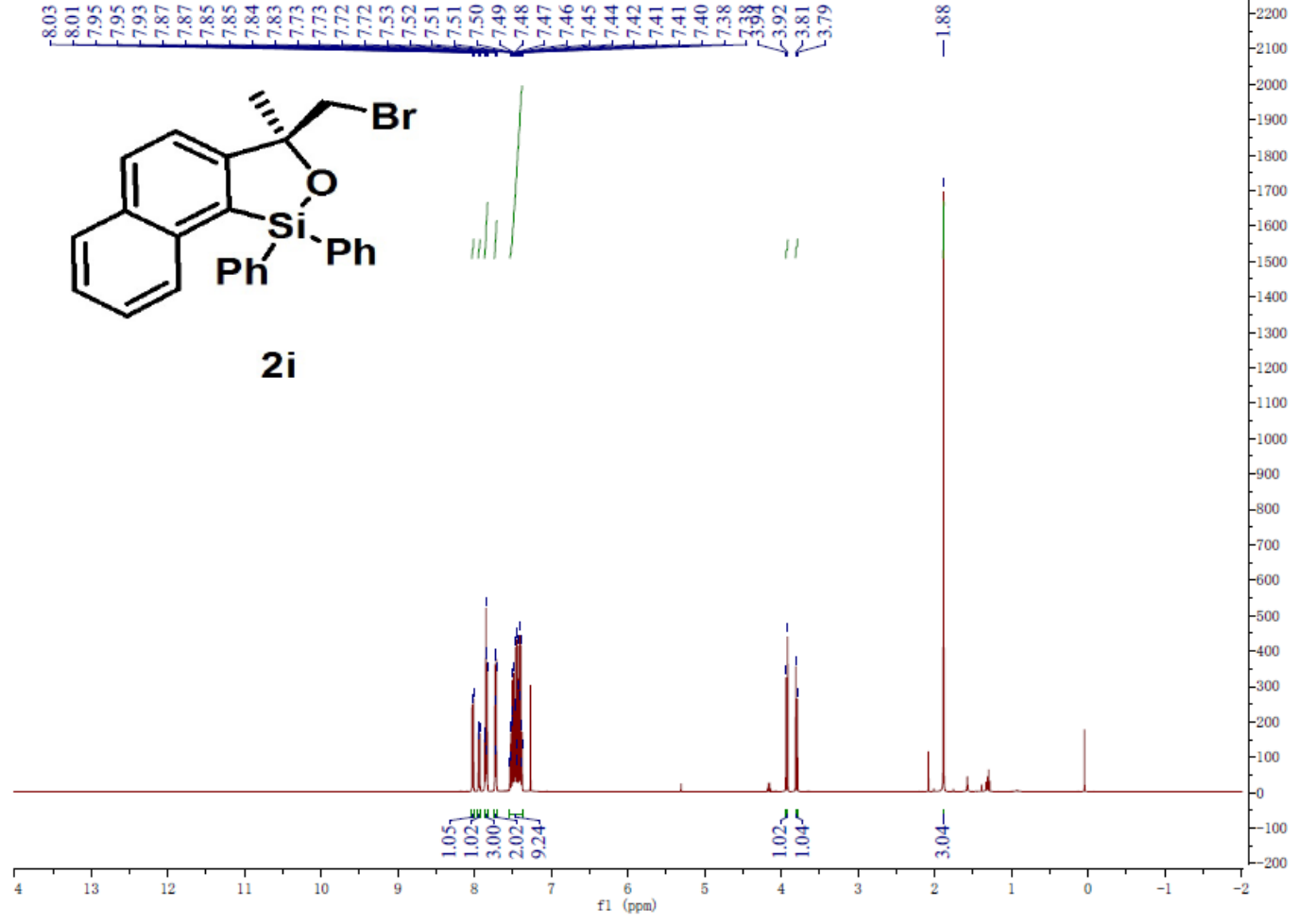

S66 

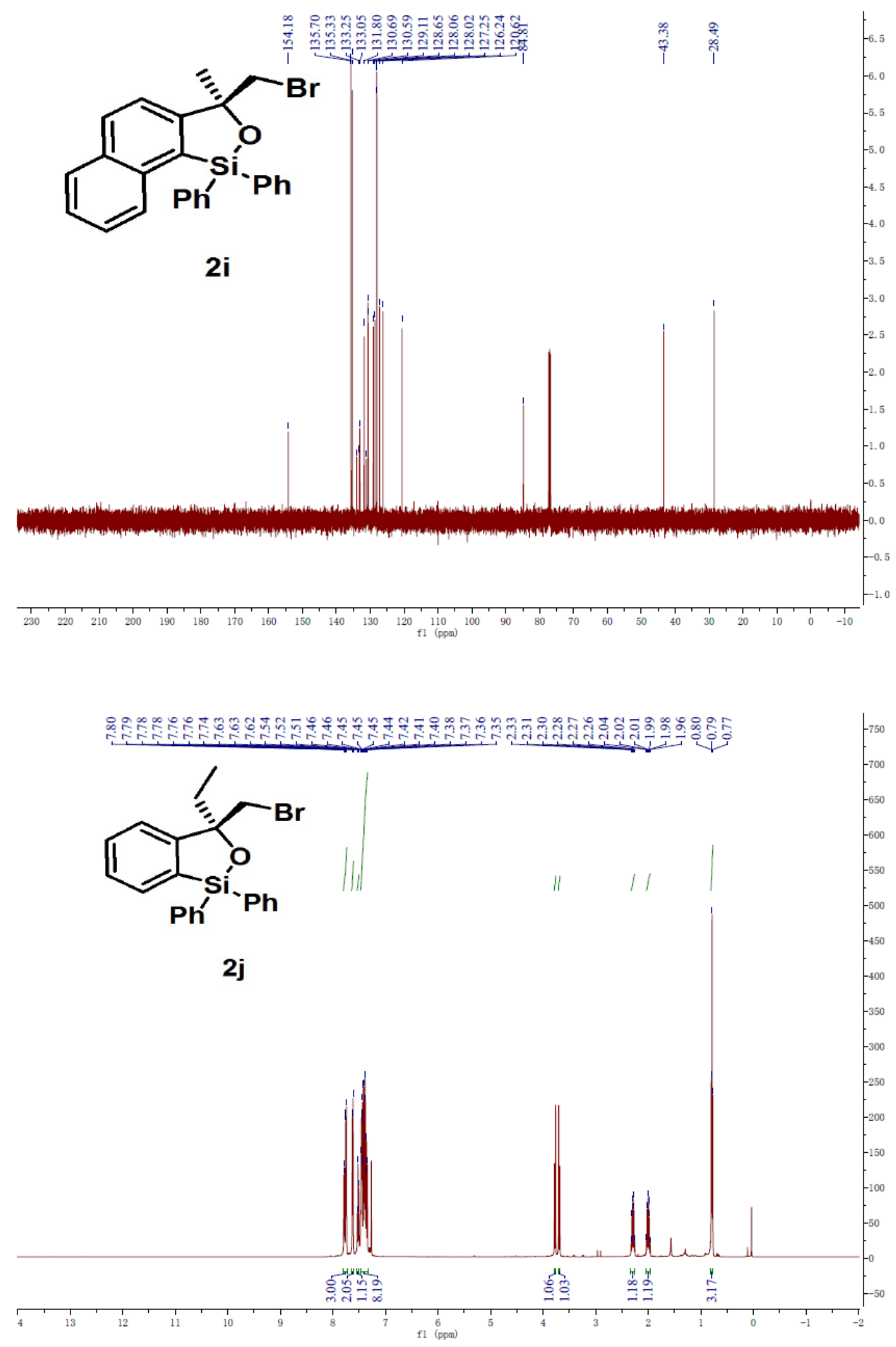

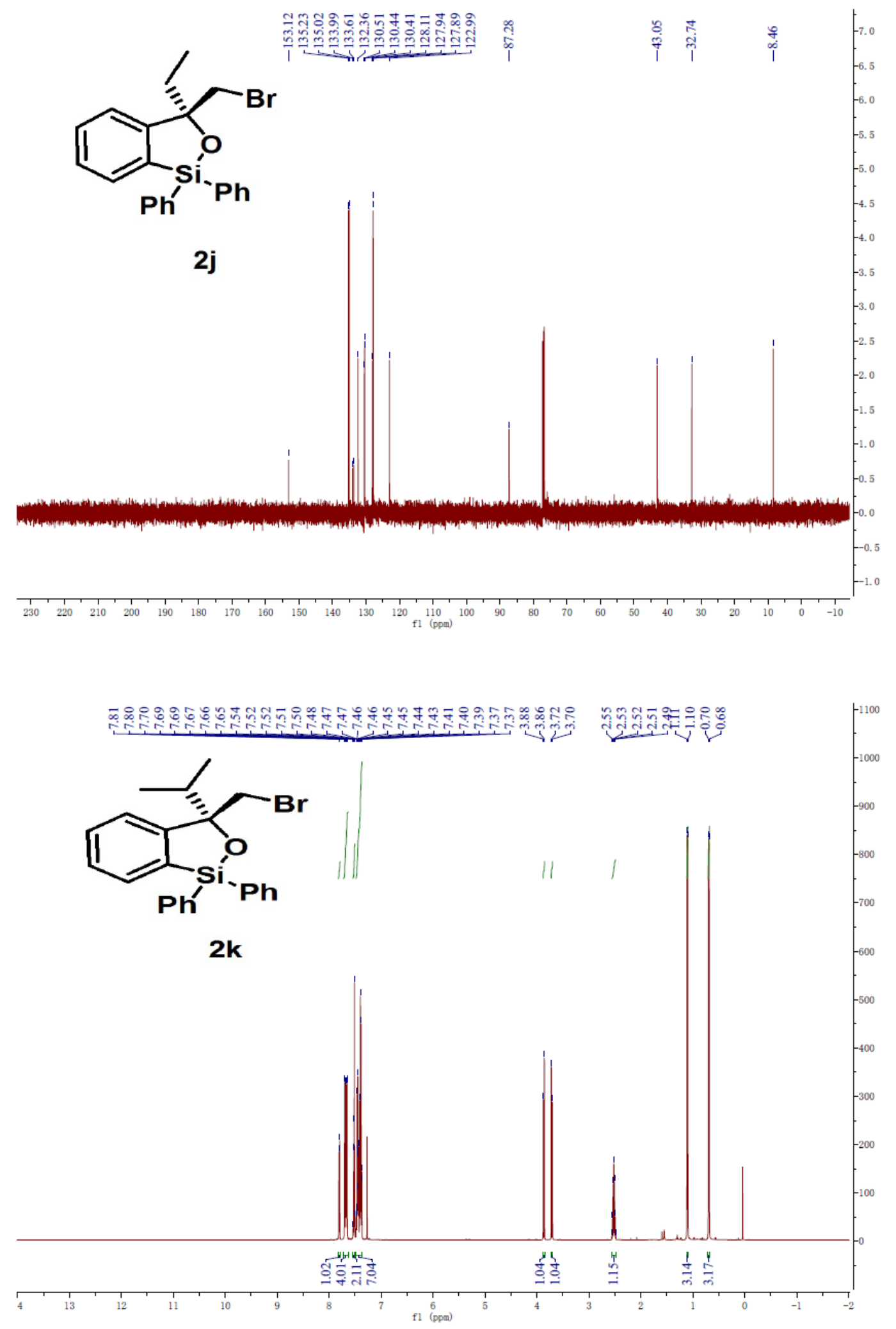

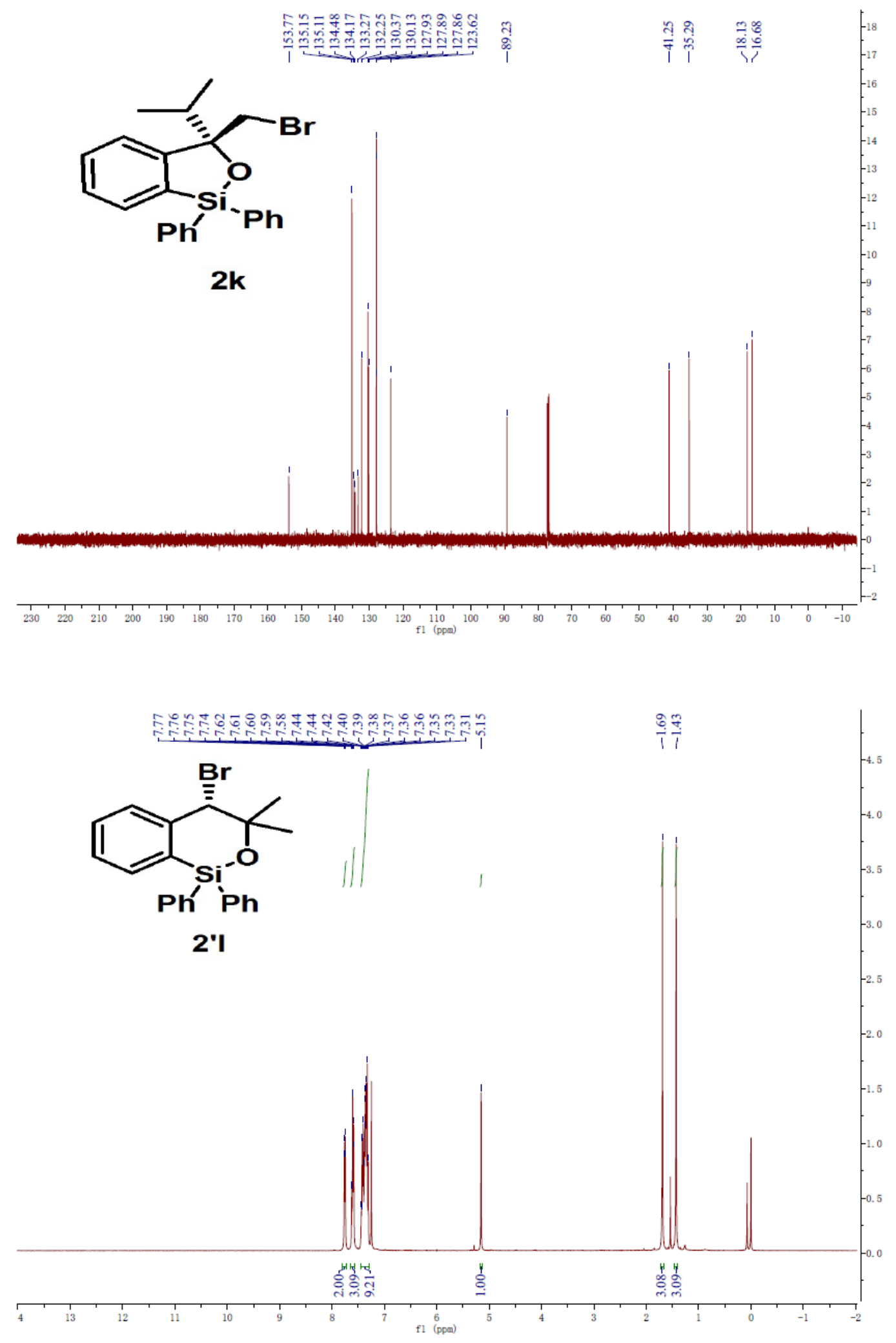

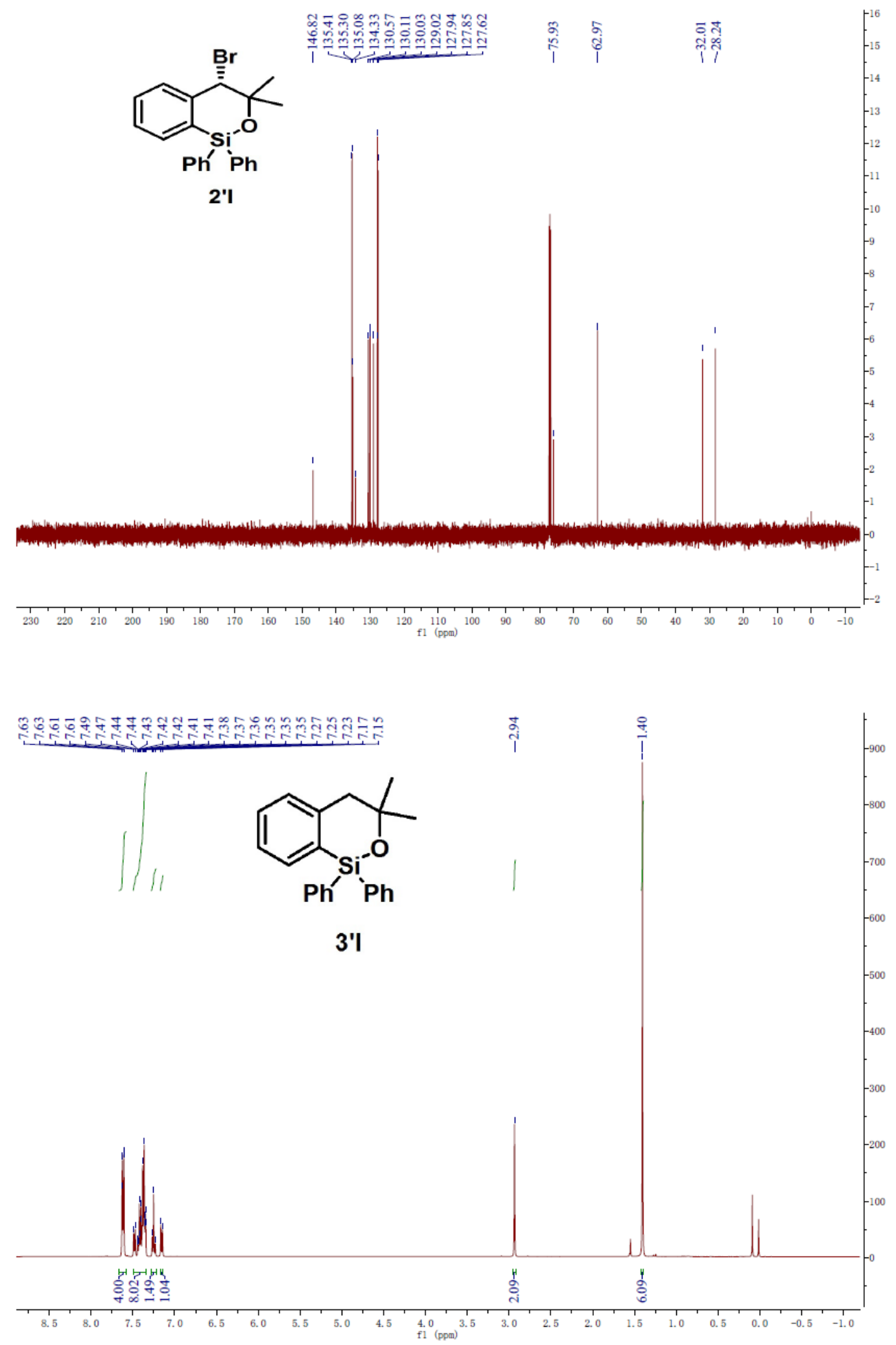

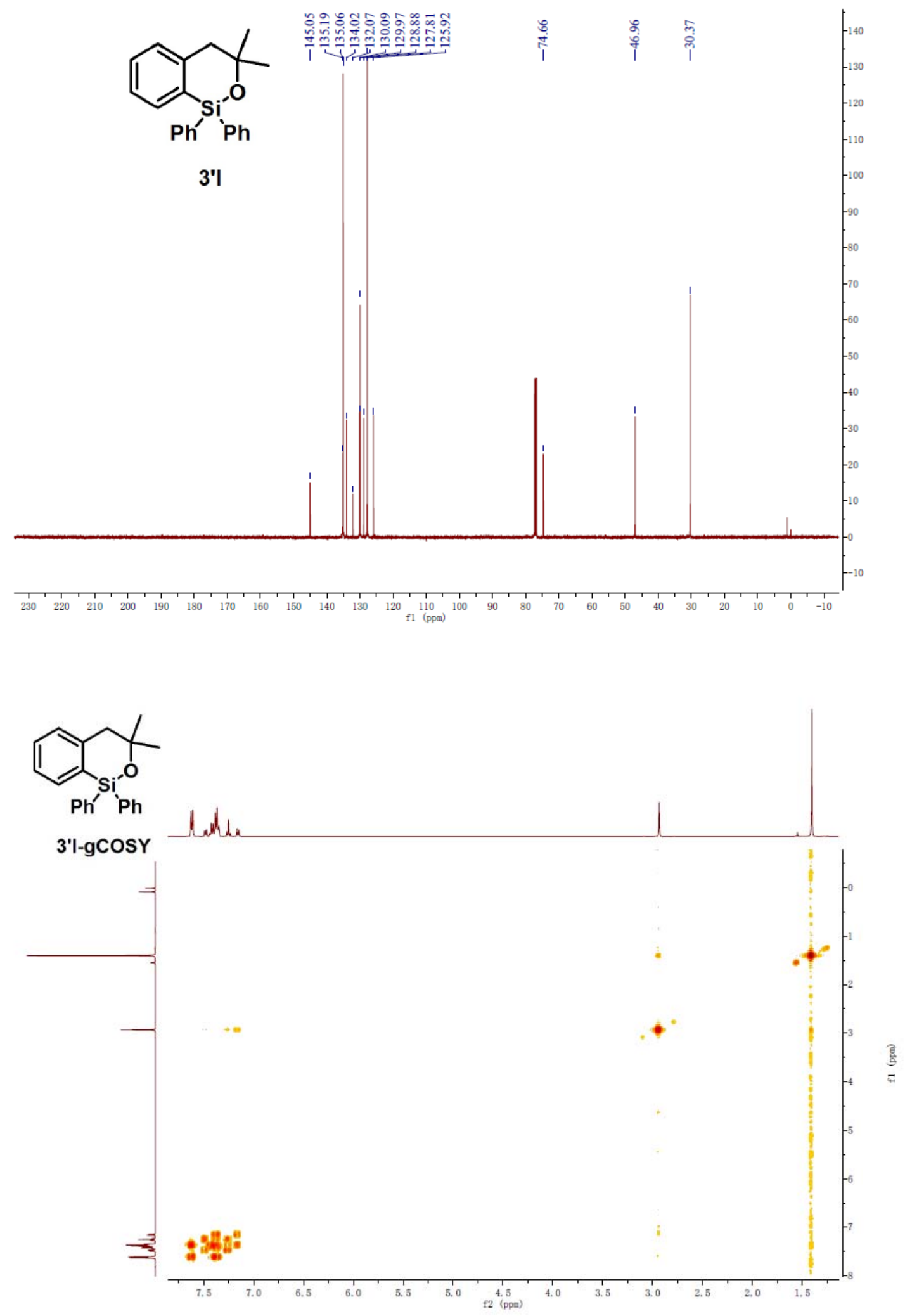

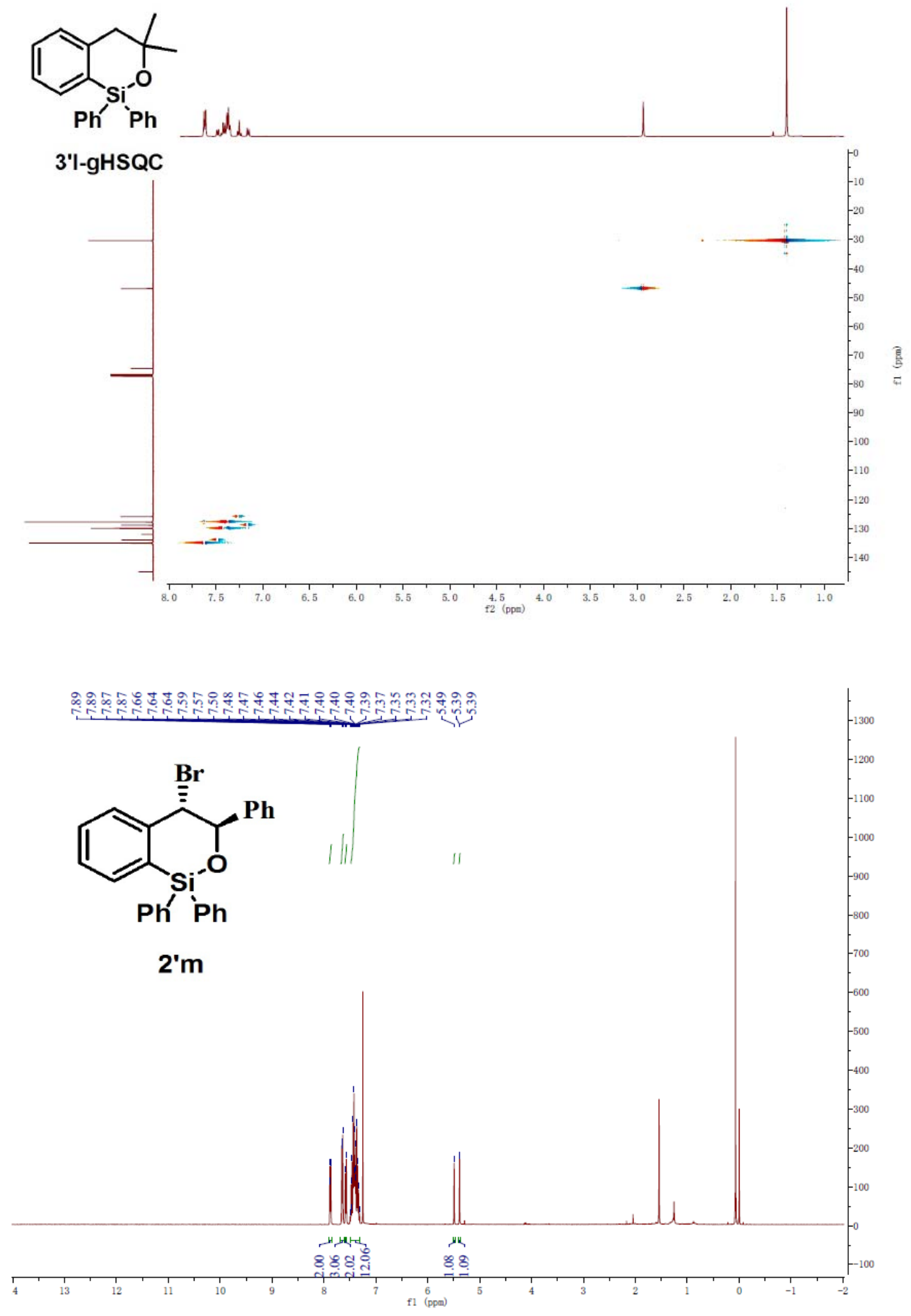

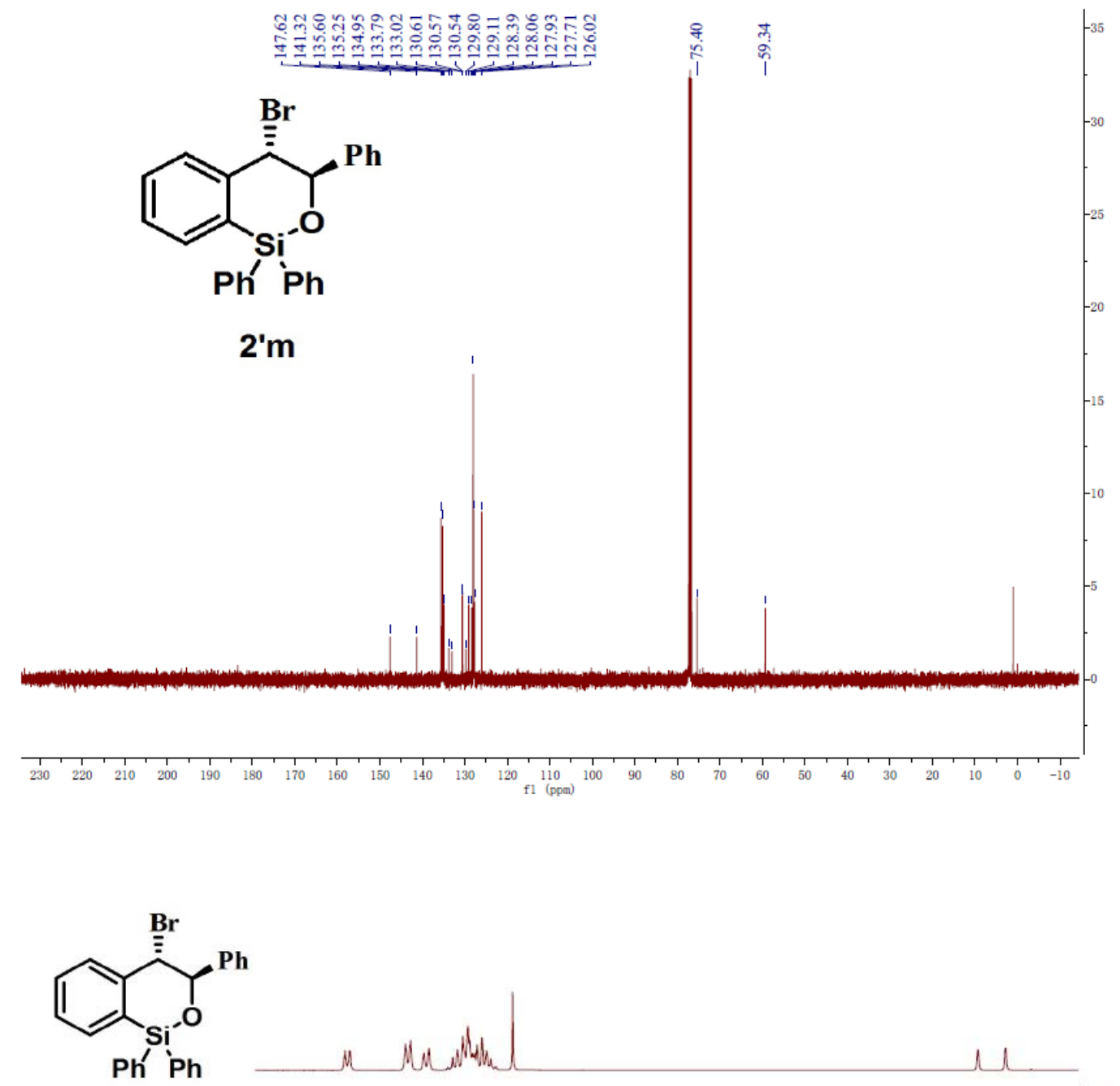

2'm-gcosy

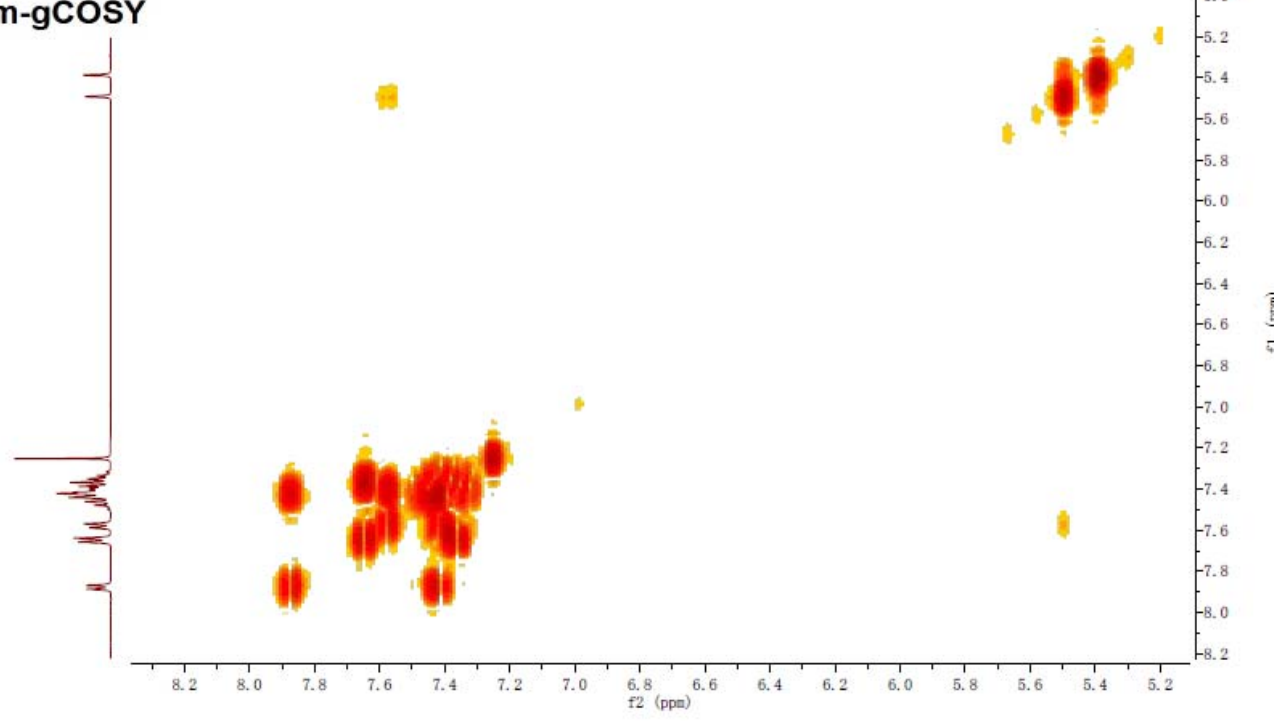




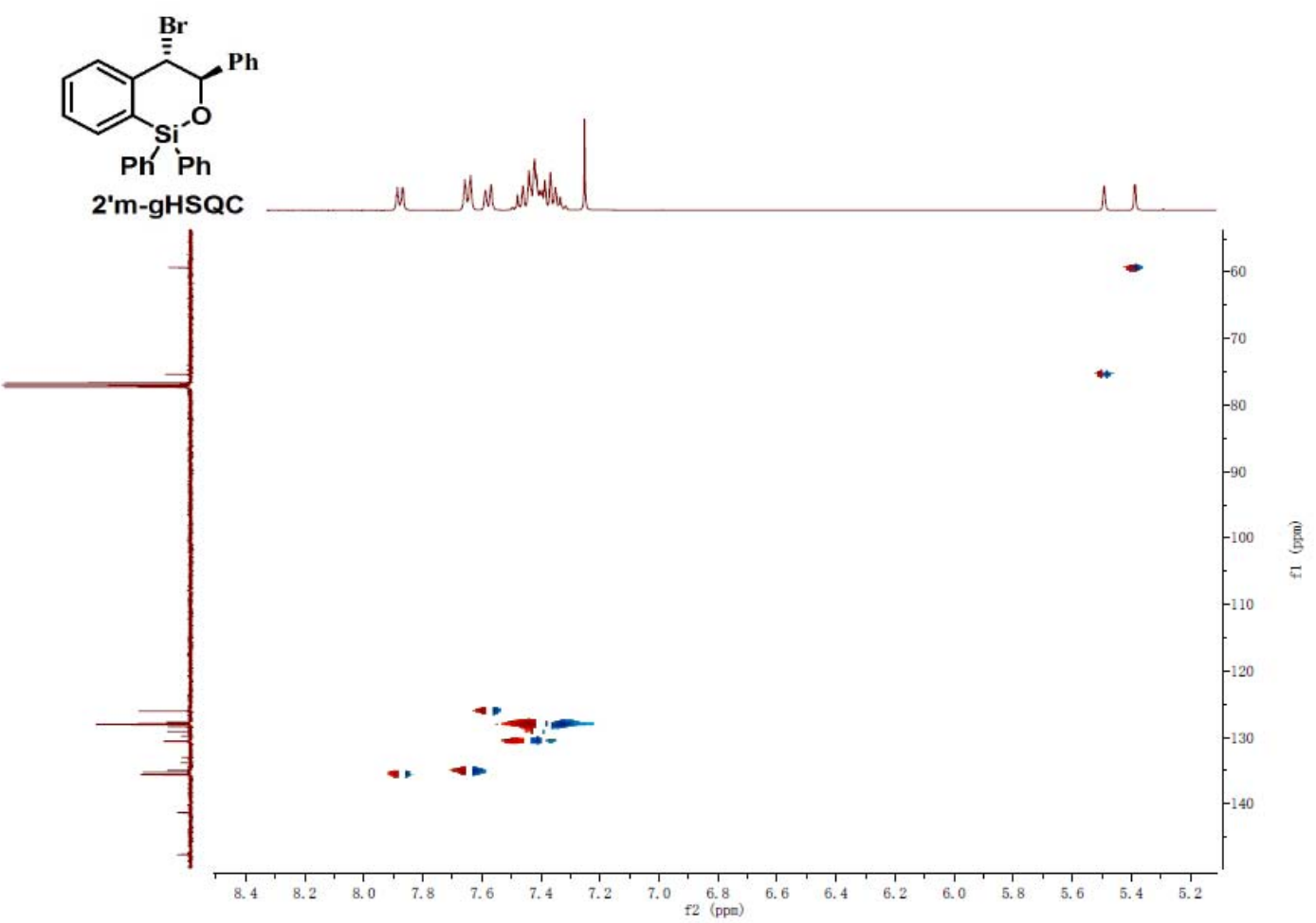

$\overbrace{\mathrm{Si}_{\mathrm{Si}}^{\mathrm{O}} \mathrm{Ph}_{\mathrm{Ph}}^{\mathrm{Br}}}^{\mathrm{Br}}$

2'm-NOESY

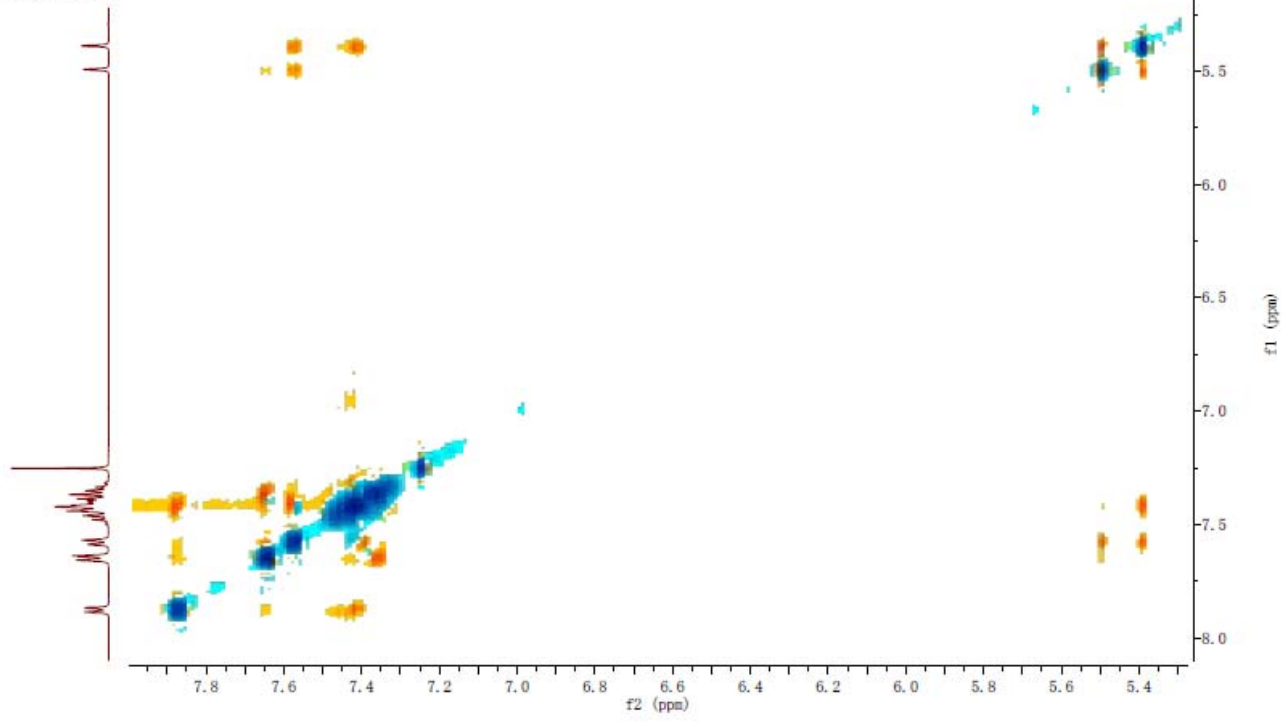



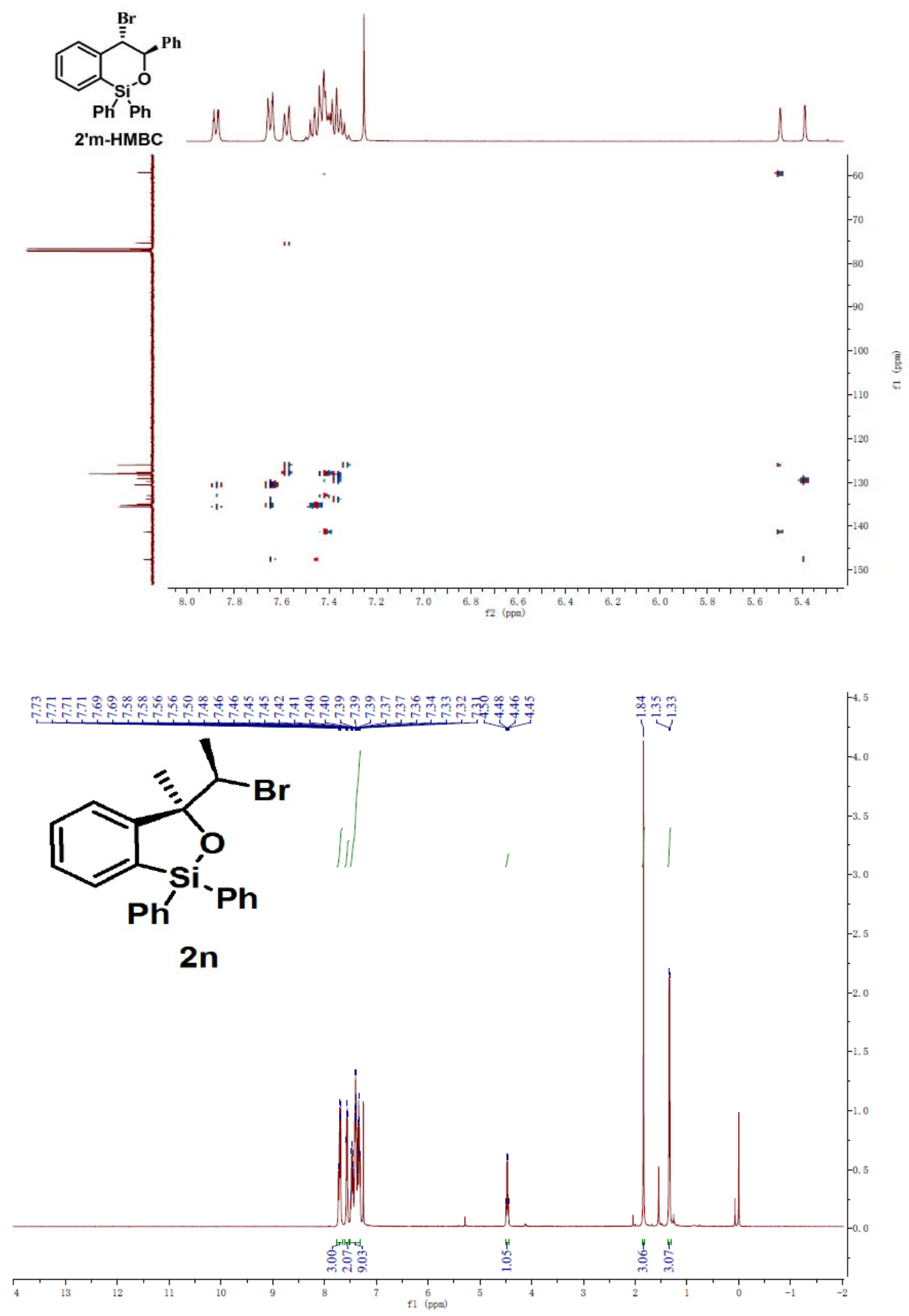

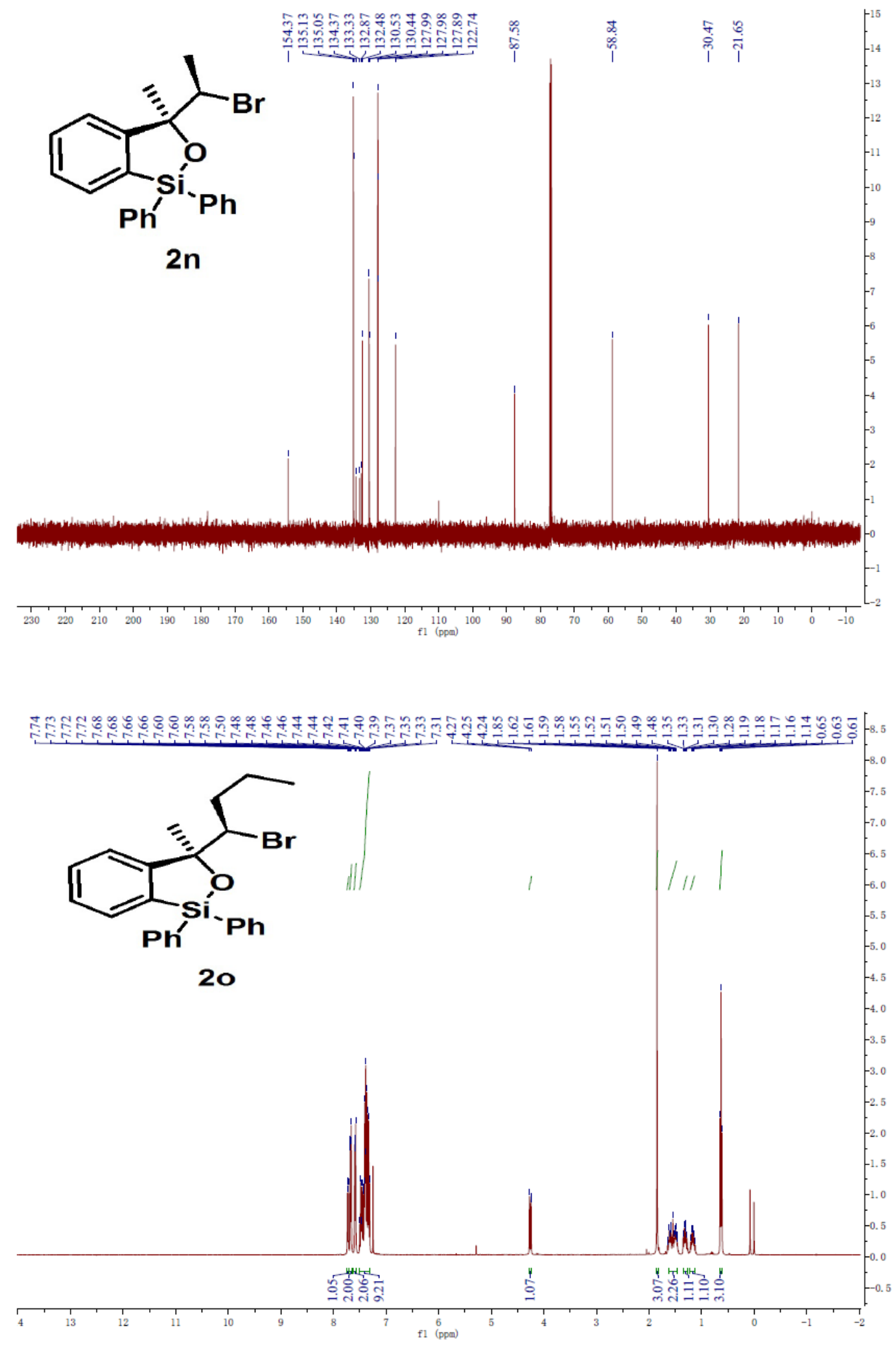

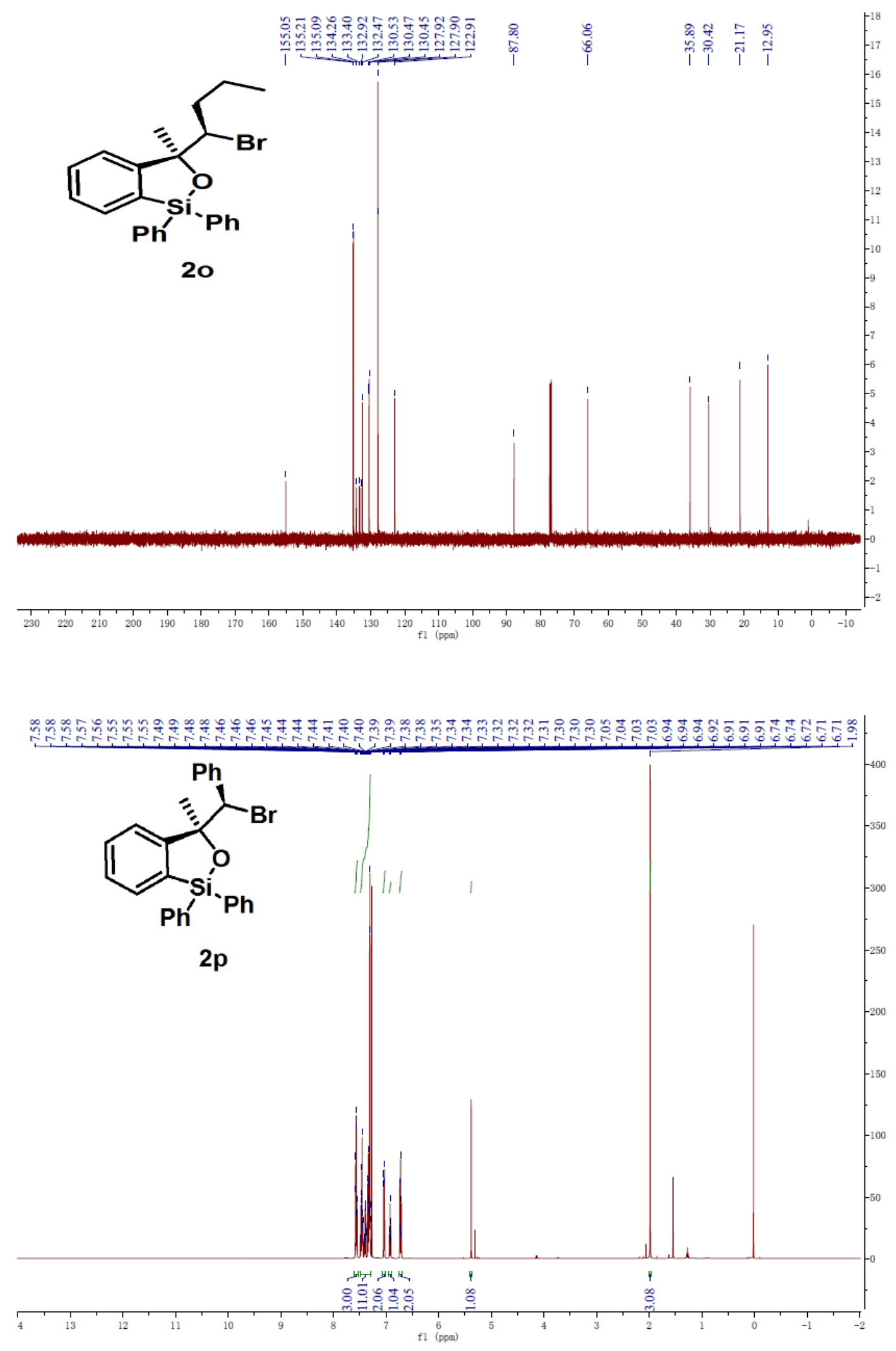

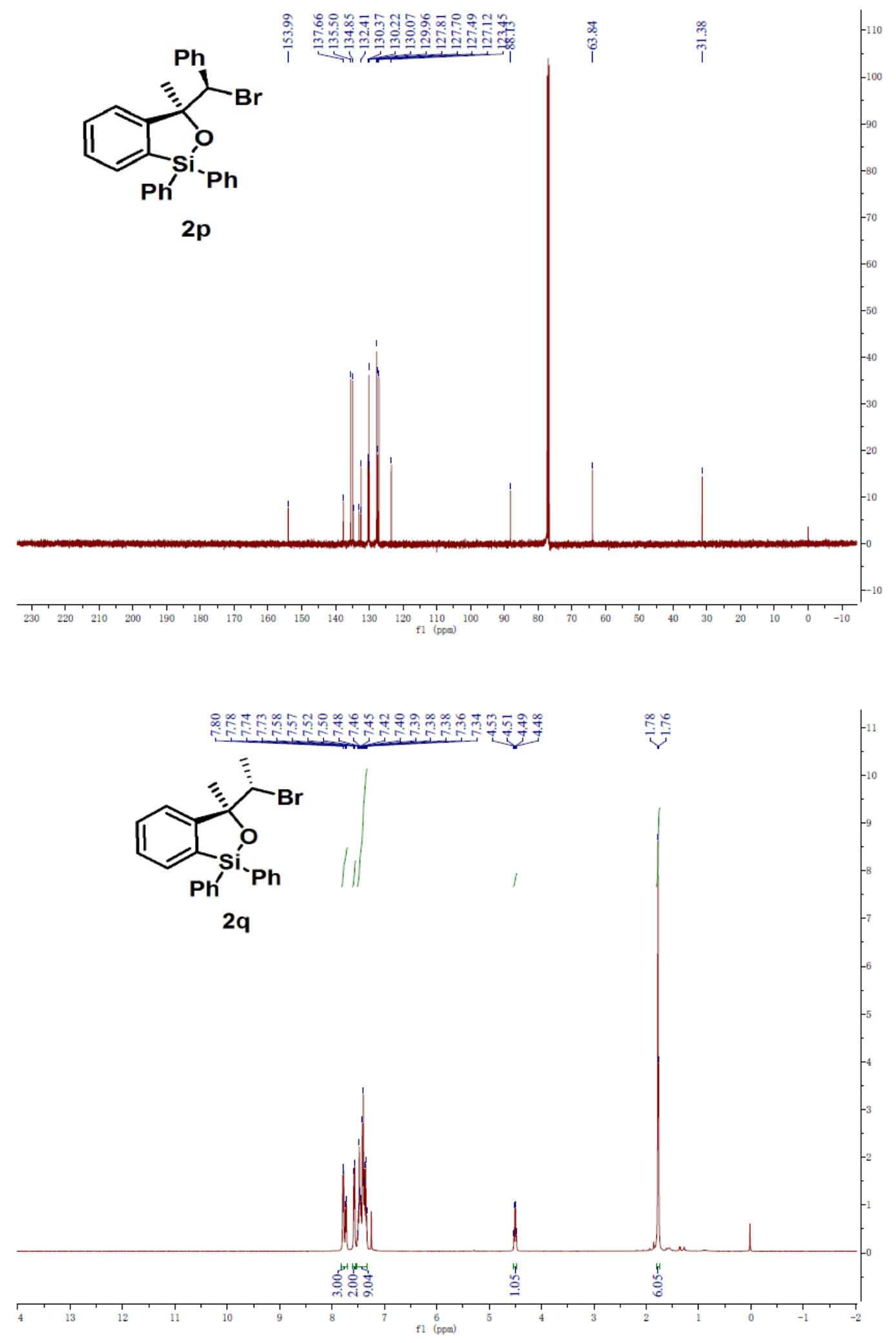

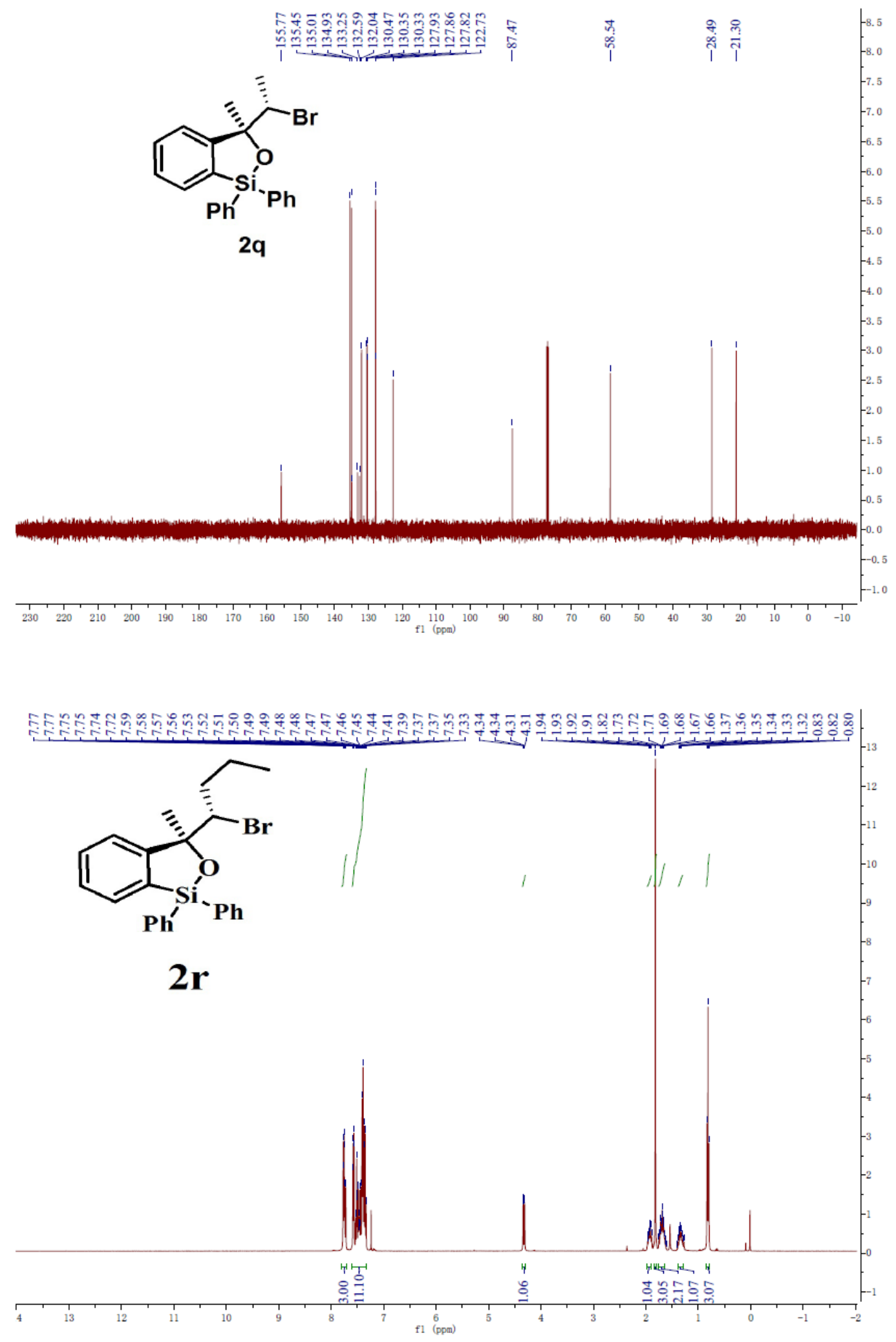

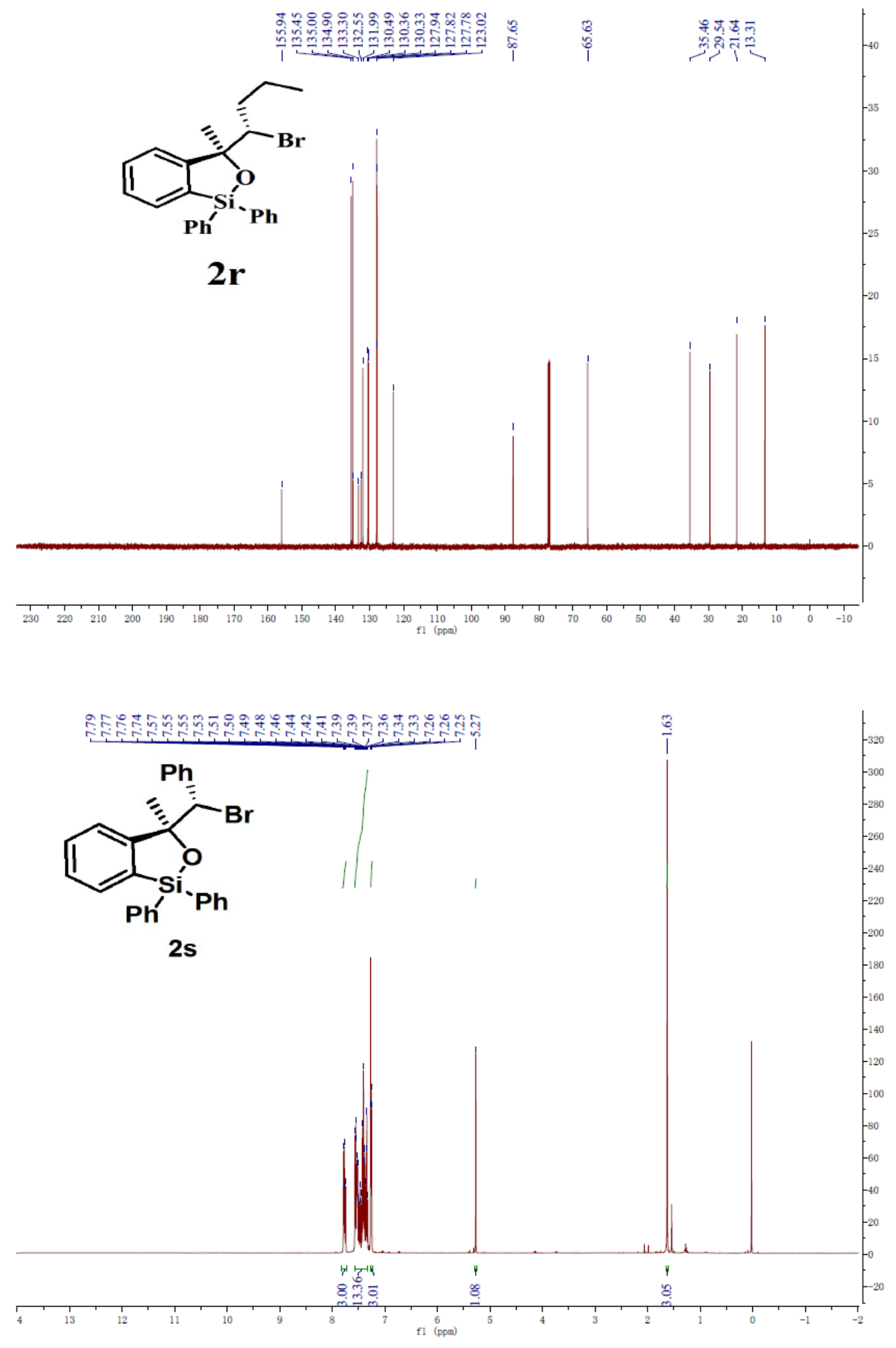

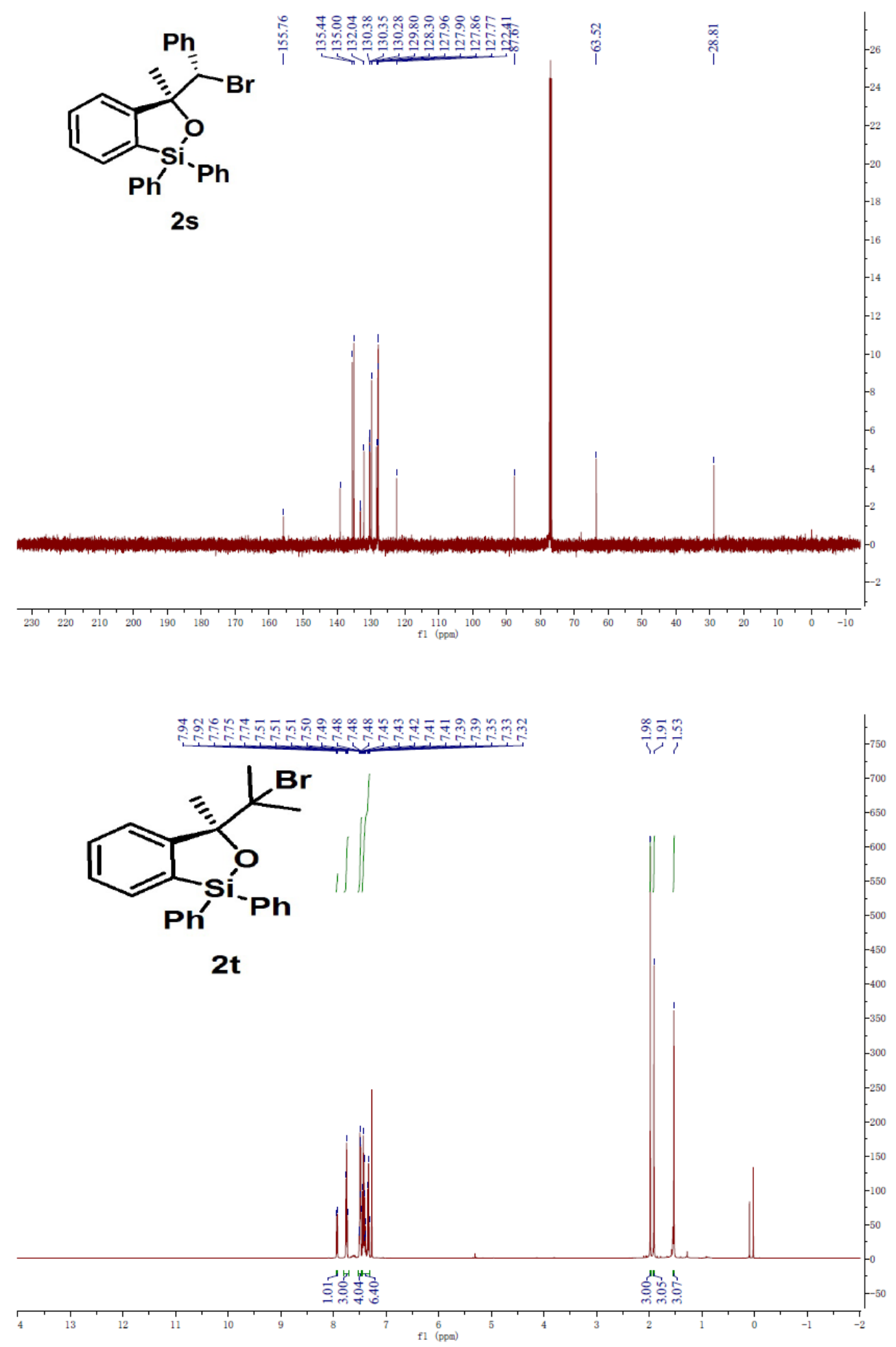

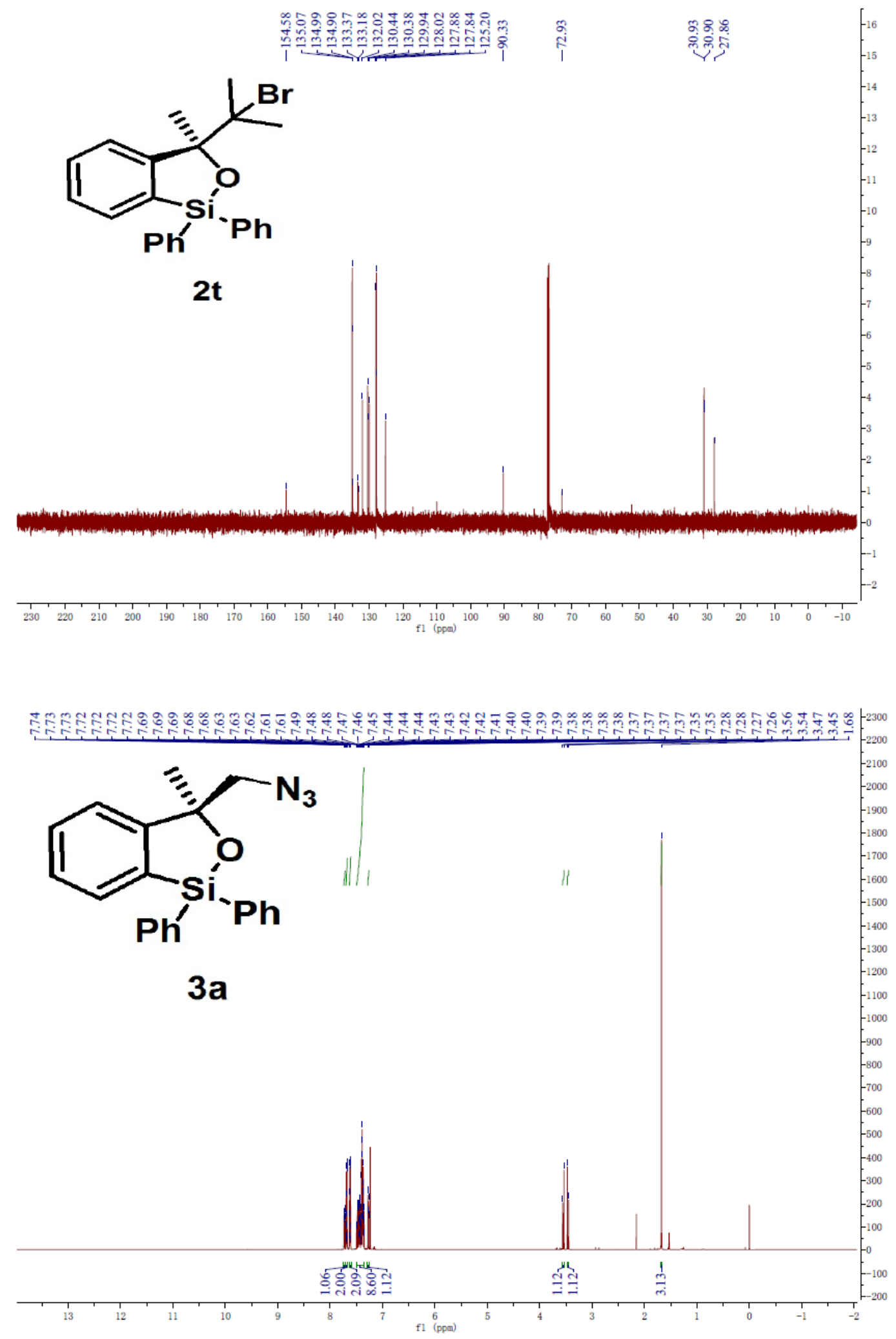

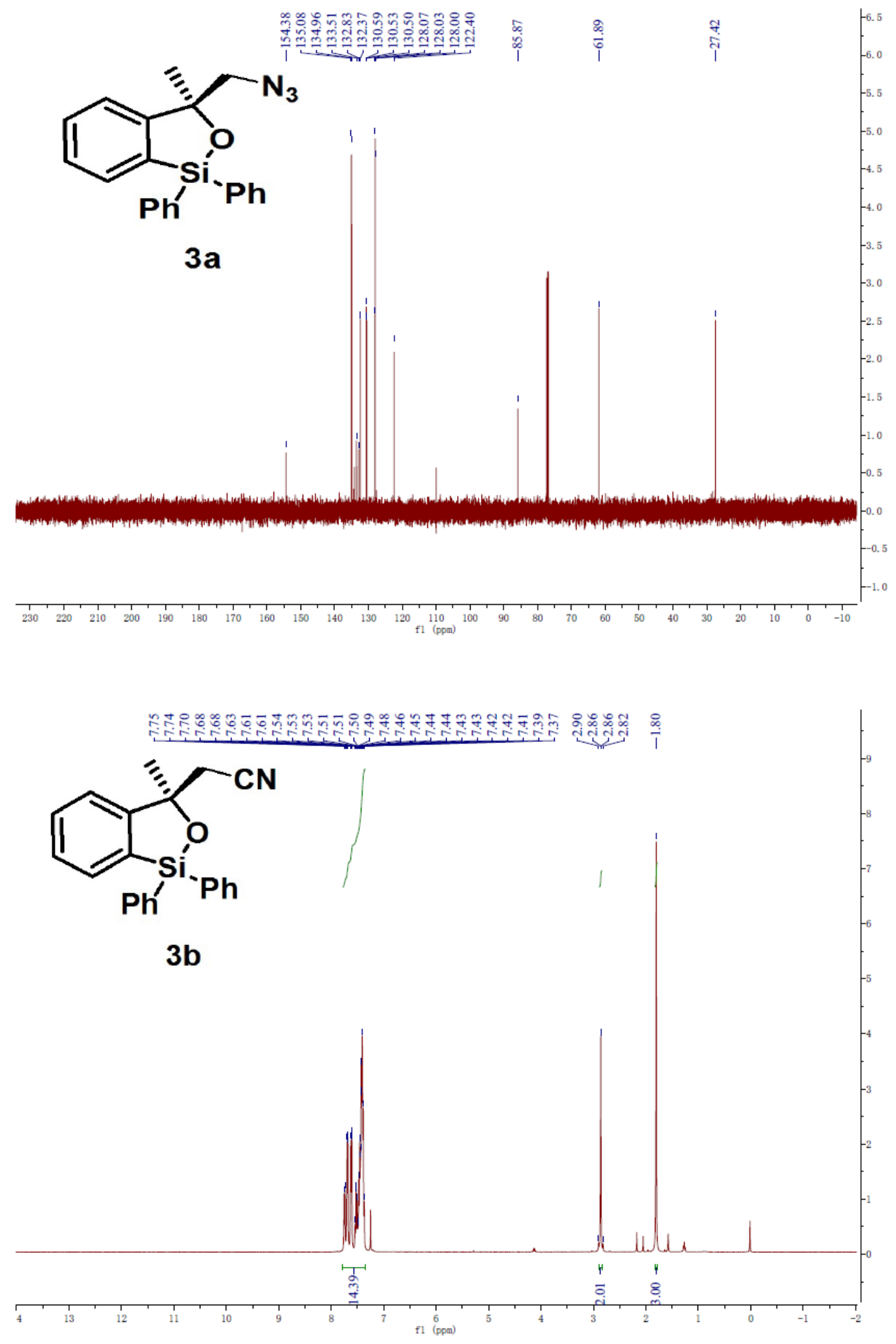

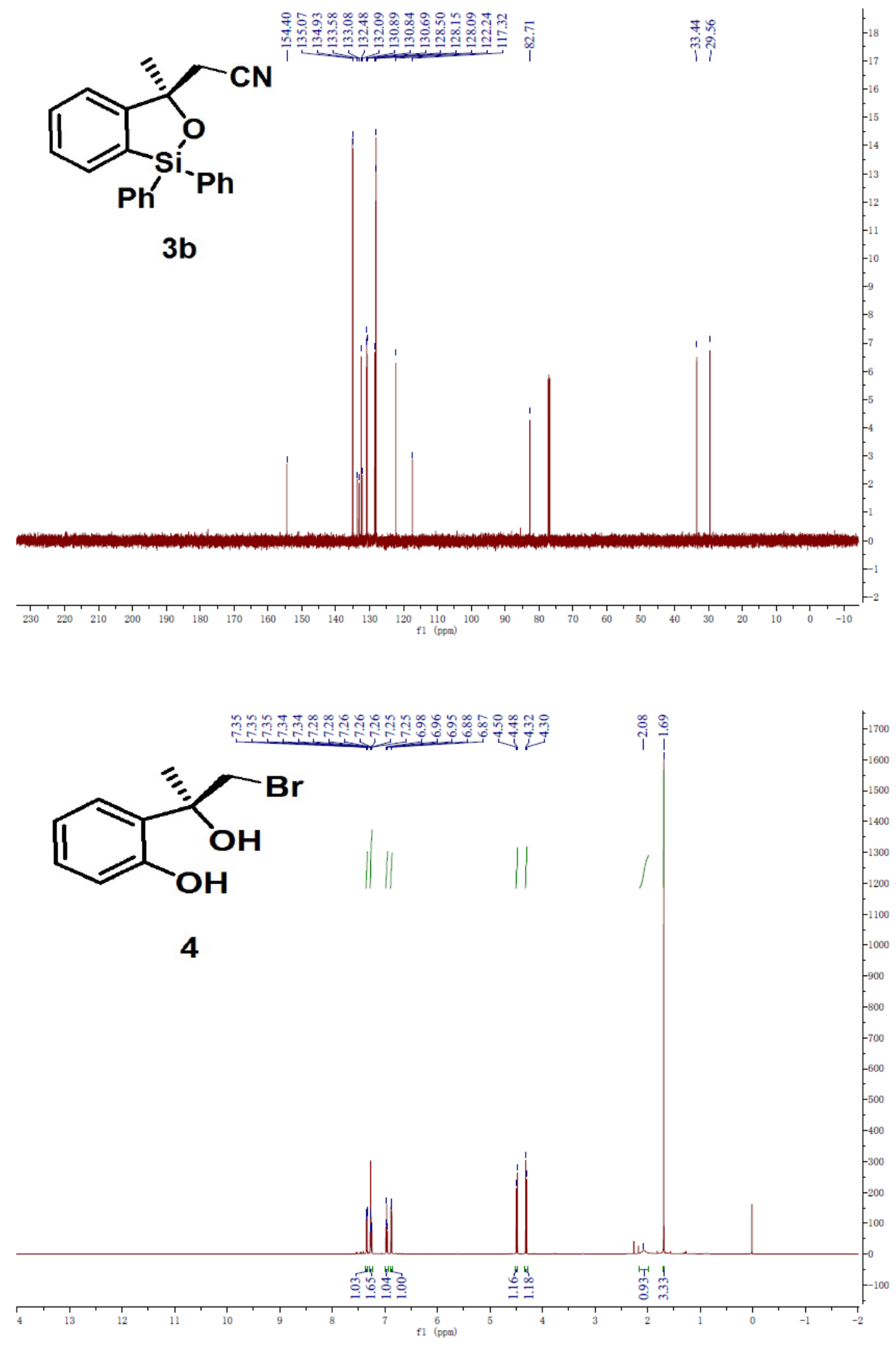

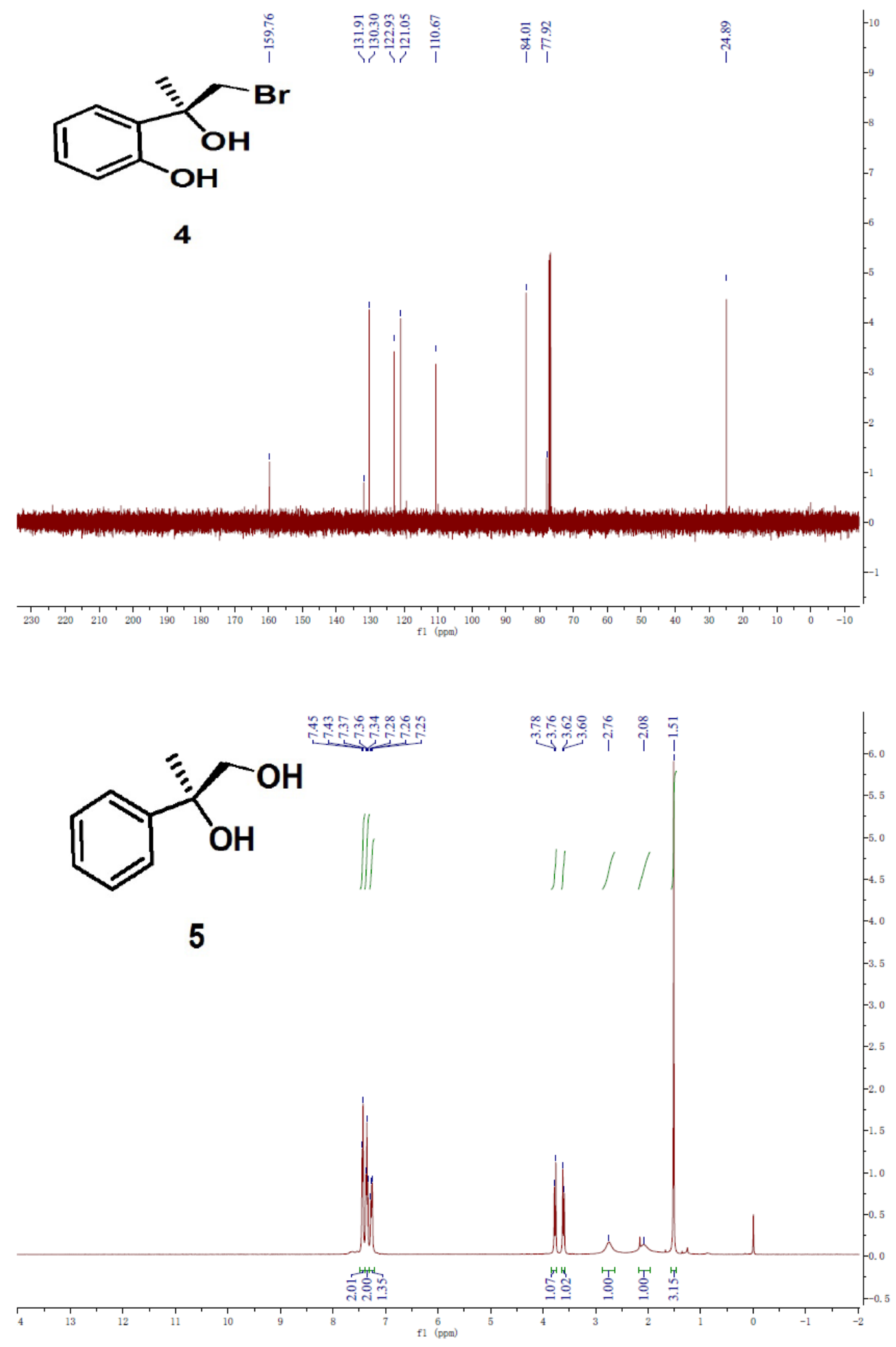

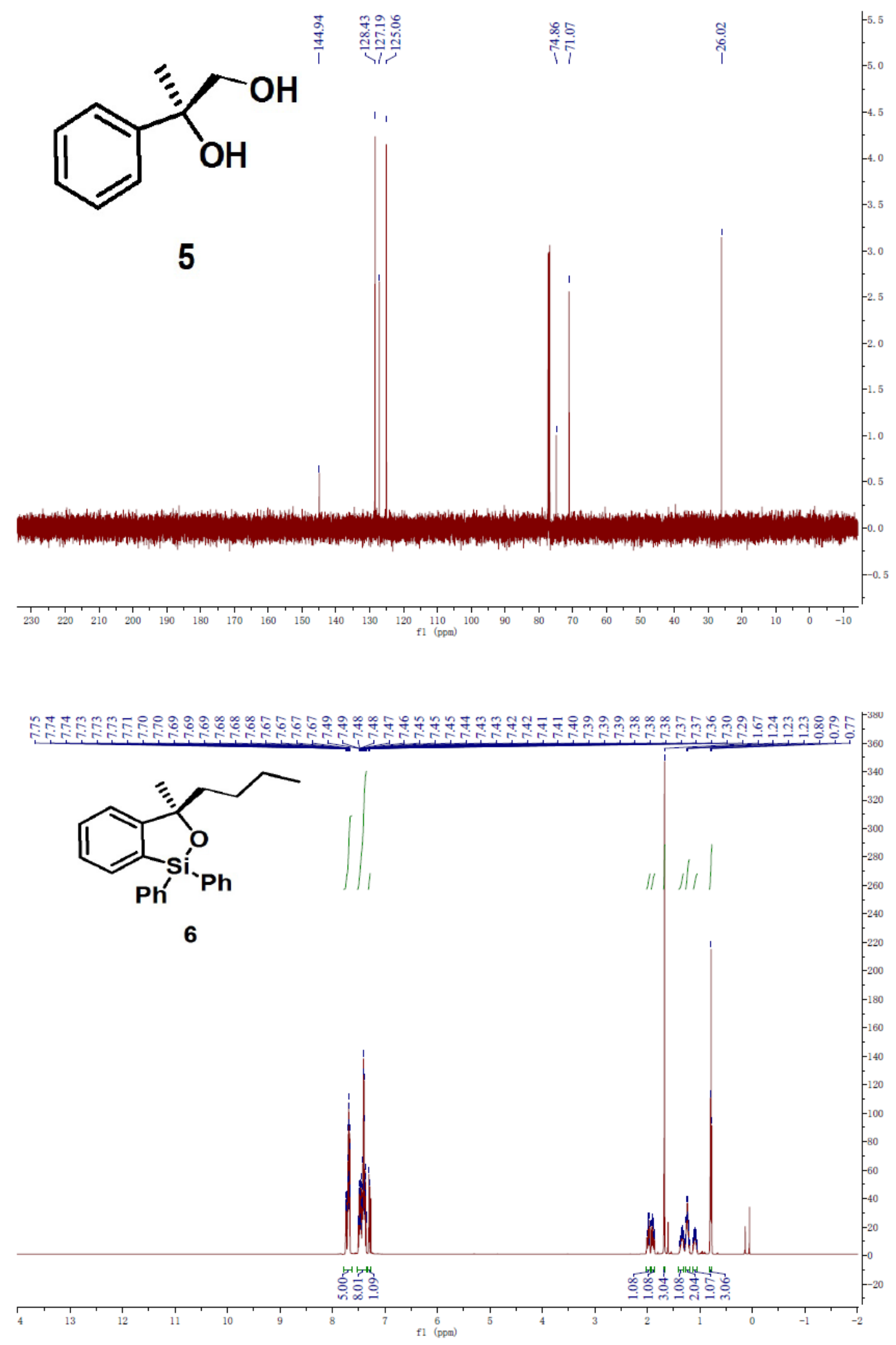

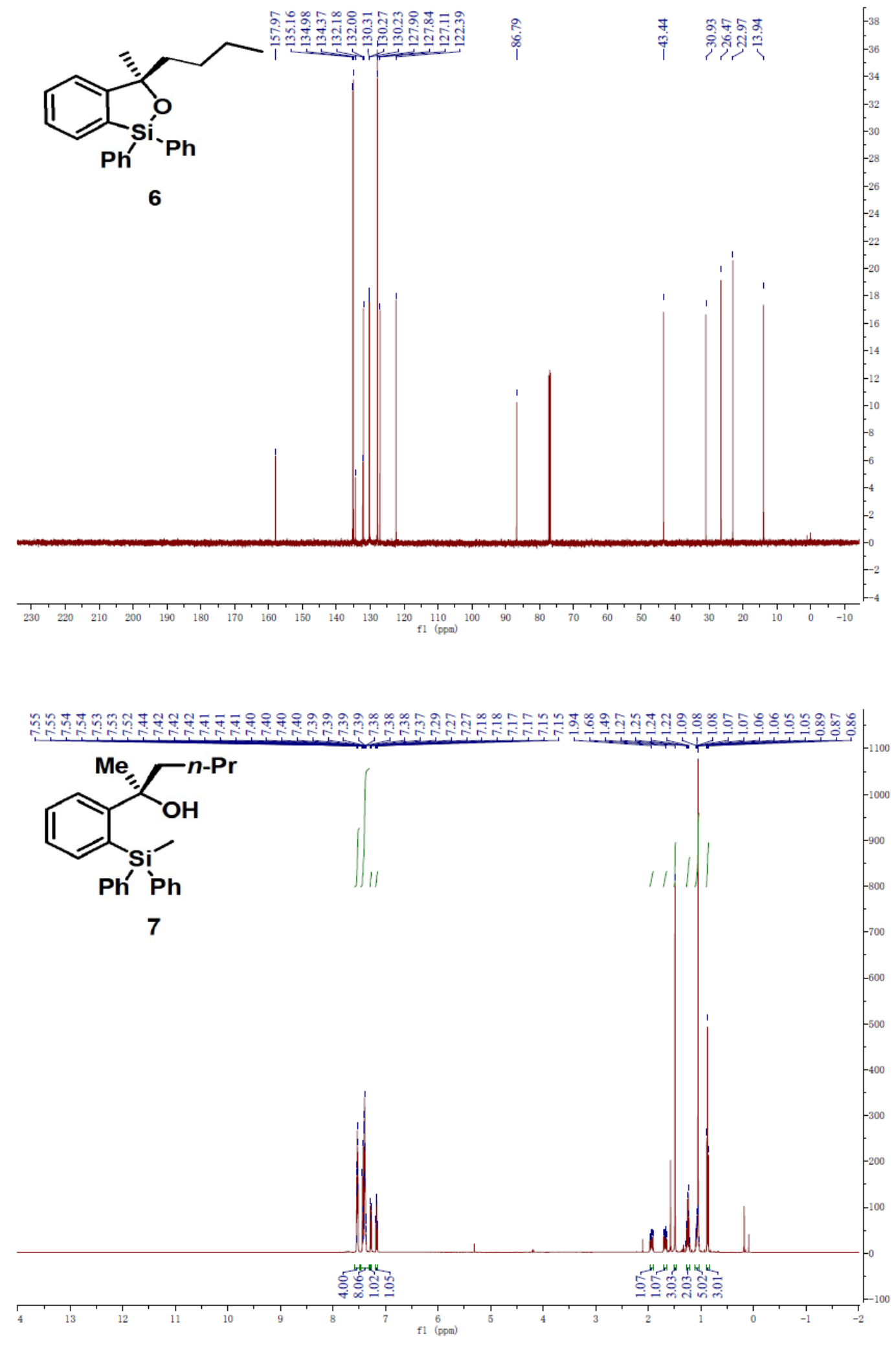

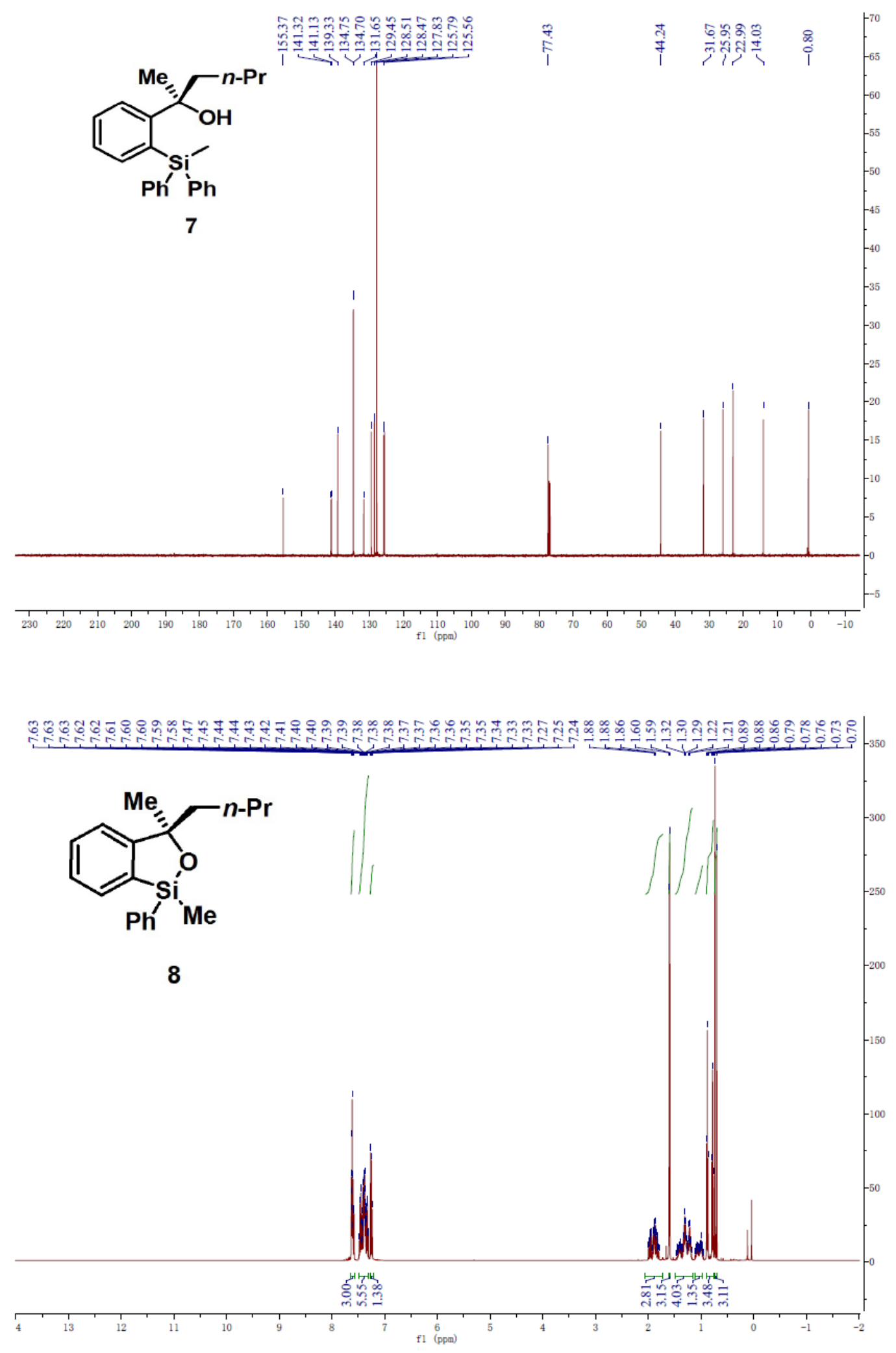


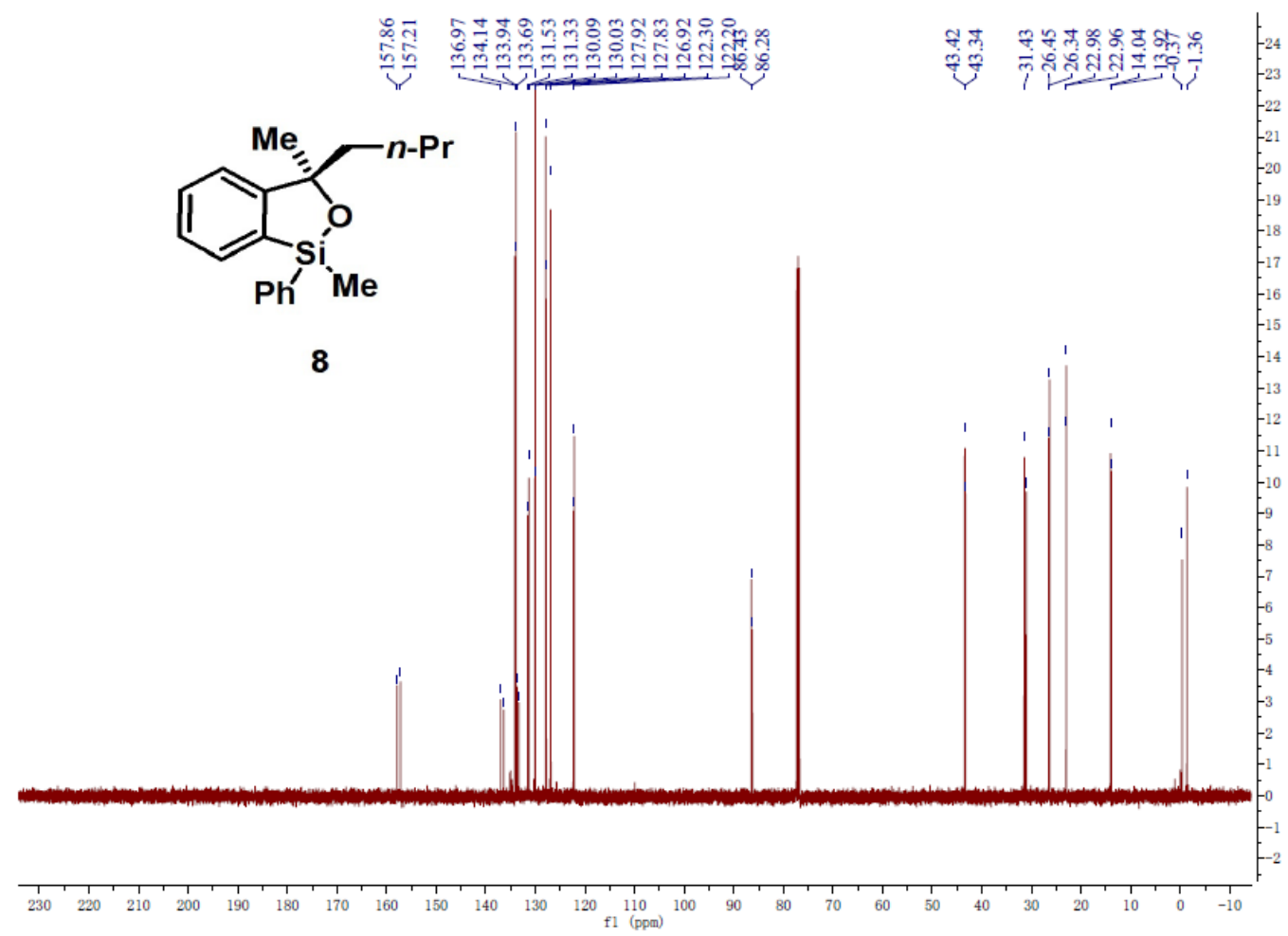




\section{HPLC Reports}

\begin{tabular}{|llll|}
\hline 2095 XZL-1A+- OD-H 9912140.3 & & \\
& & & \\
\hline Sample Name: & XZL-1A+- OD-H 9912140.3 & Injection Volume: & 6.0 \\
Vial Number: & RE1 & Channet & UV_VIS_1 \\
Sample Type: & unknown & Wavelength: & 214 \\
Control Program: & WXL-2014-2 & Bandwidth: & n.a. \\
Quantif. Method: & WXL & Dilution Factor: & 1.0000 \\
Recording Time: & $2014 / 11 / 11$ 21:11 & Sample Weight & 1.0000 \\
Run Time (min): & 22.88 & Sample Amount: & 1.0000 \\
\hline
\end{tabular}

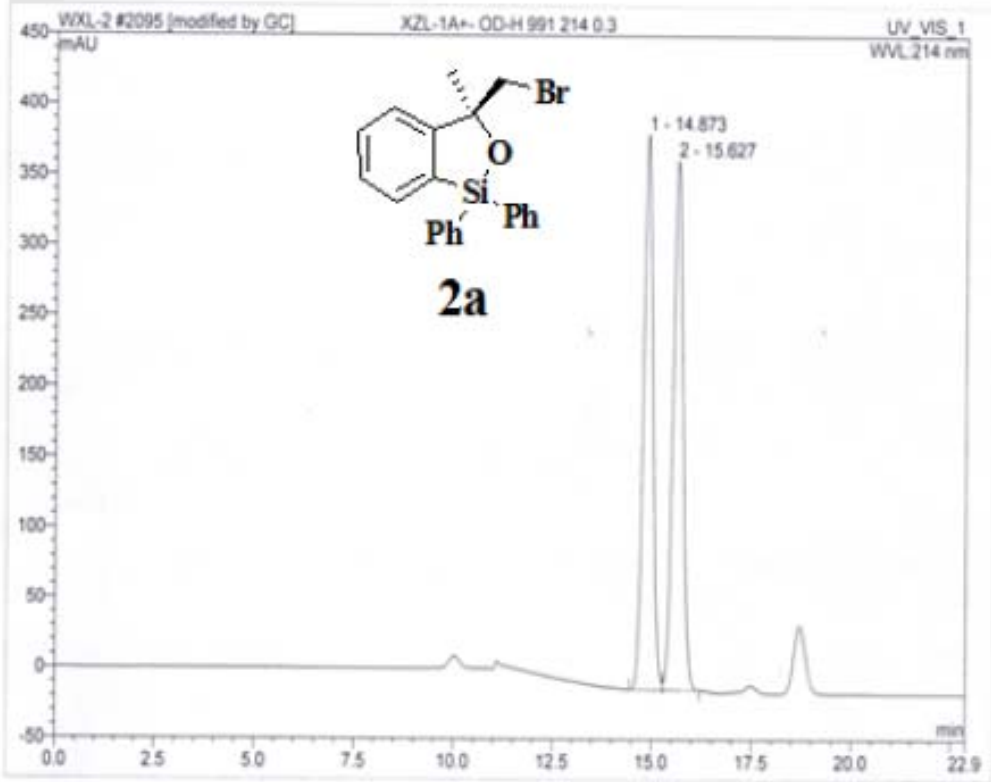

\begin{tabular}{|c|c|c|c|c|c|c|c|}
\hline No. & $\begin{array}{c}\text { Ret. Time } \\
\text { min }\end{array}$ & Peak Name & $\begin{array}{c}\text { Height } \\
\text { mAU }\end{array}$ & $\begin{array}{c}\text { Area } \\
\text { mAU"min }\end{array}$ & $\begin{array}{c}\text { Rel.Area } \\
\%\end{array}$ & Amount & Type \\
\hline 1 & 1487 & n.a. & 394.543 & 108024 & 49.98 & na & BM \\
\hline 2 & 1563 & na & 376429 & 108093 & 5002 & na & MB \\
\hline Total: & & & 770.972 & 216.117 & 10000 & 0.000 & \\
\hline
\end{tabular}




\begin{tabular}{|llll|}
\hline \multicolumn{5}{|c|}{ OD-H 9912140.3} & \\
& & & \\
\hline Sample Name: & XZL-2073-3A 0O-H 9912140.3 & Injection Volume: & 5.0 \\
Vial Number: & BE3 & Channe: & UV_VIS_1 \\
Sample Type: & unknown & Waveliength: & 214 \\
Control Program: & WXL-2014-2 & Bandwidth: & n.a. \\
Quantif: Method: & WXL & Dilution Factor: & 1.0000 \\
Recording Time: & $2014 / 12 / 15$ 10:31 & Sample Weight: & 1.0000 \\
Run Time (min): & 26.49 & Sample Amount: & 1.0000 \\
\hline
\end{tabular}

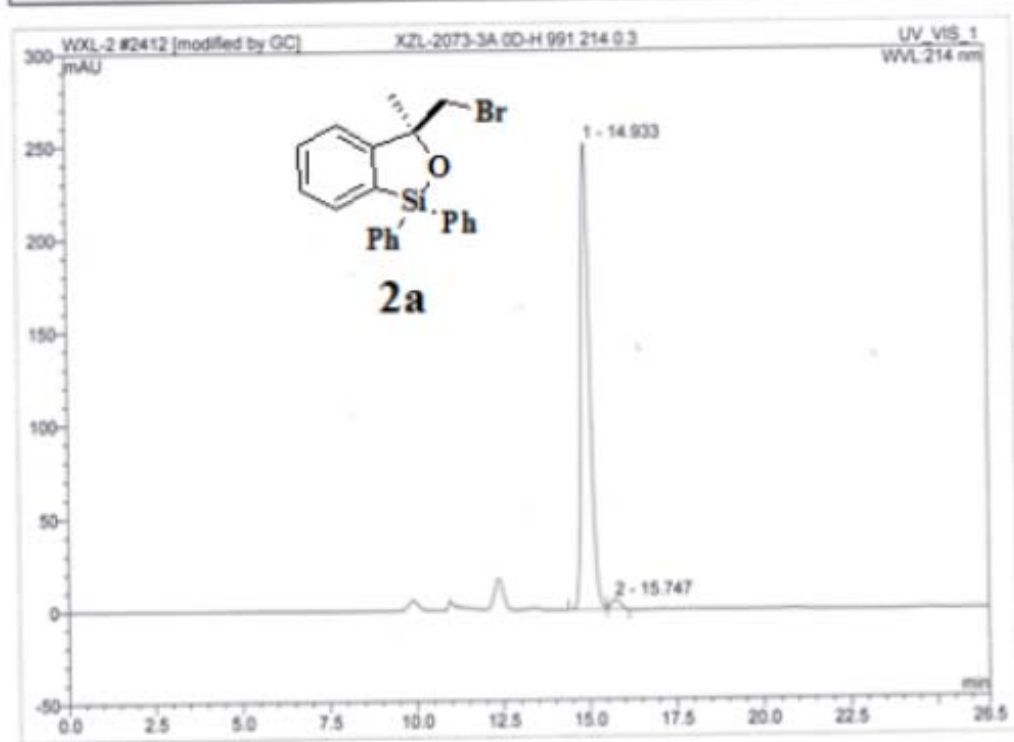

\begin{tabular}{|c|c|c|c|c|c|c|c|}
\hline No. & $\begin{array}{c}\text { Ret. Time } \\
\text { min }\end{array}$ & Peak Name & $\begin{array}{c}\text { Height } \\
\text { maU }\end{array}$ & $\begin{array}{c}\text { Area } \\
\text { maurmin }\end{array}$ & $\begin{array}{c}\text { Rel.Area } \\
8\end{array}$ & Amount & Type \\
\hline 1 & 14.93 & n.a. & 251.303 & 72077 & 97.74 & na. & BM* \\
\hline 2 & 15.75 & na. & 5.484 & 1.667 & 226 & na. & $\mathrm{MB}^{*}$ \\
\hline Total: & & & 256.787 & 73.745 & 10000 & 0.000 & \\
\hline
\end{tabular}




\begin{tabular}{|c|c|c|c|}
\hline \multicolumn{4}{|c|}{$\begin{array}{c}2097 \times Z L-2 A+- \text { OD-H } 9552140.7 \\
\text { 2064 }\end{array}$} \\
\hline $\begin{array}{l}\text { Sample Name: } \\
\text { Vial Number }\end{array}$ & $X Z L-2 A *-O D-H 9552140.7$ & Injection Volume: & 2.0 \\
\hline Vial Number: & RE2 & Channet: & UV_VIS_1 \\
\hline Sample Type: & unknown & Wavelength: & 214 \\
\hline Control Program & WXL-2014-2 & Bandwidth & n.a. \\
\hline Quantil. Method: & $w X L$ & Dilution Factor: & 1.0000 \\
\hline Recording Time: & 2014/11/11 16:31 & Sample Weight: & 1.0000 \\
\hline Run Time (min): & 25.00 & Sample Amount: & 1.0000 \\
\hline
\end{tabular}

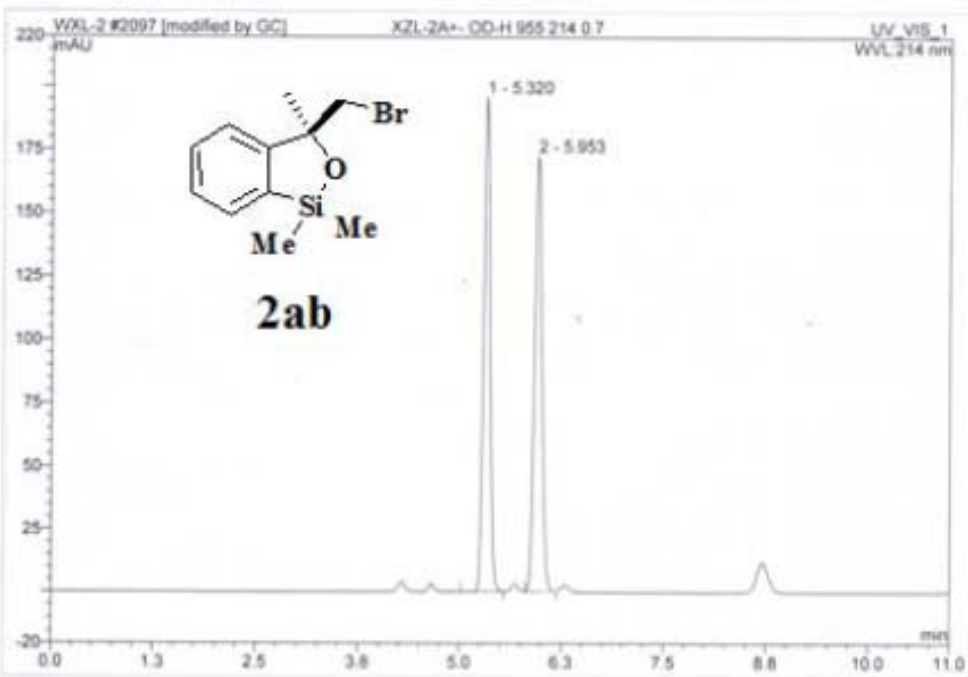

\begin{tabular}{|c|c|c|c|c|c|c|c|}
\hline No. & $\begin{array}{c}\text { Ret. Time } \\
\text { min }\end{array}$ & Peak Name & $\begin{array}{c}\text { Height } \\
\text { mau }\end{array}$ & $\begin{array}{c}\text { Area } \\
\text { mAu'min }\end{array}$ & $\begin{array}{c}\text { Rel.Area } \\
\%\end{array}$ & Amount & Type \\
\hline 1 & 5.32 & na. & 195918 & 17.984 & 49.96 & na & Bens: \\
\hline 2 & 5.95 & na & 172523 & 18014 & 50.04 & na & BMB: \\
\hline Total: & & & 368.441 & 35.999 & 100.00 & 0.000 & \\
\hline
\end{tabular}




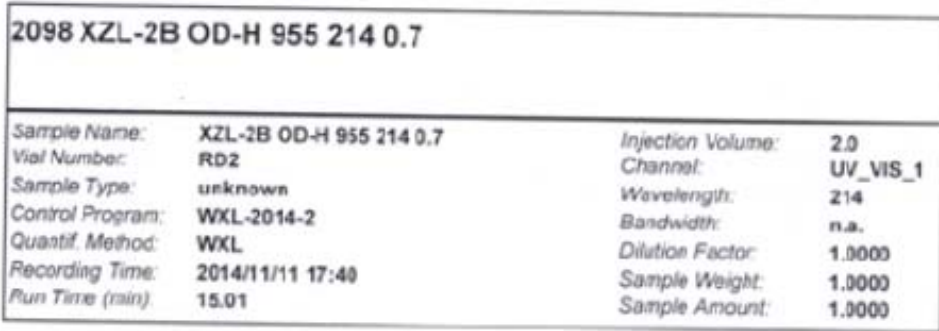

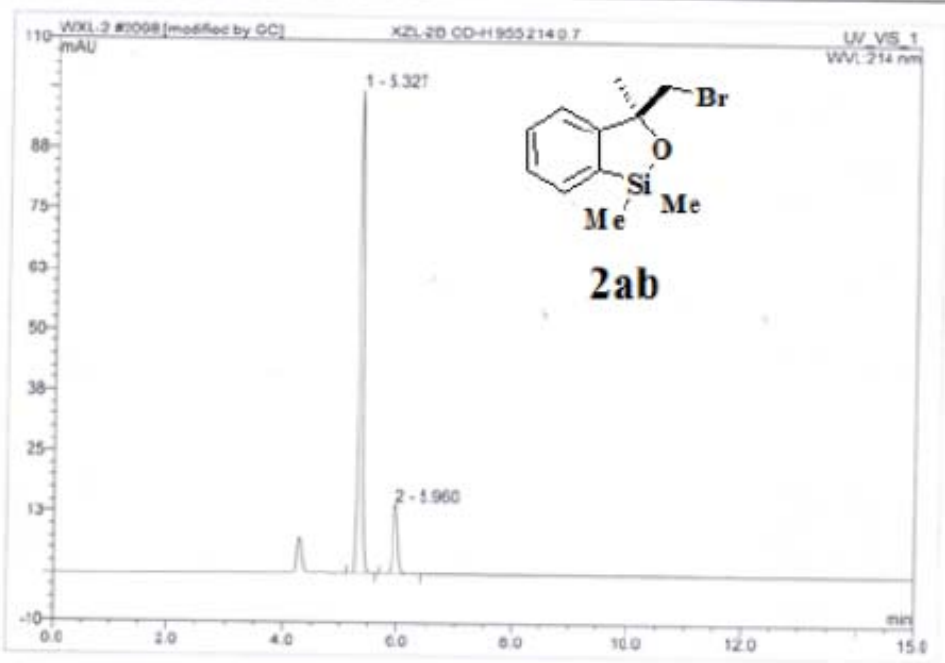

\begin{tabular}{|c|c|c|c|c|c|c|c|}
\hline No. & $\begin{array}{c}\text { Ret. Time } \\
\text { min }\end{array}$ & Peak Name & $\begin{array}{c}\text { Height } \\
\text { maU }\end{array}$ & $\begin{array}{c}\text { Area } \\
\text { mAU*min }\end{array}$ & $\begin{array}{c}\text { Rel.Ares } \\
\times\end{array}$ & Amount & Туре \\
\hline 1 & 5.33 & na. & 99840 & 9.191 & 85.93 & n.a. & BMB' \\
\hline 2 & 5.96 & $n a$ & 14442 & 1.505 & 1607 & na & $8 M B^{\circ}$ \\
\hline Total: & & & 114282 & 10.605 & 100.00 & 0.000 & \\
\hline
\end{tabular}




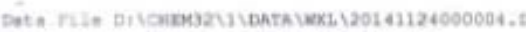

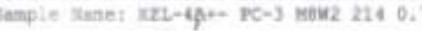
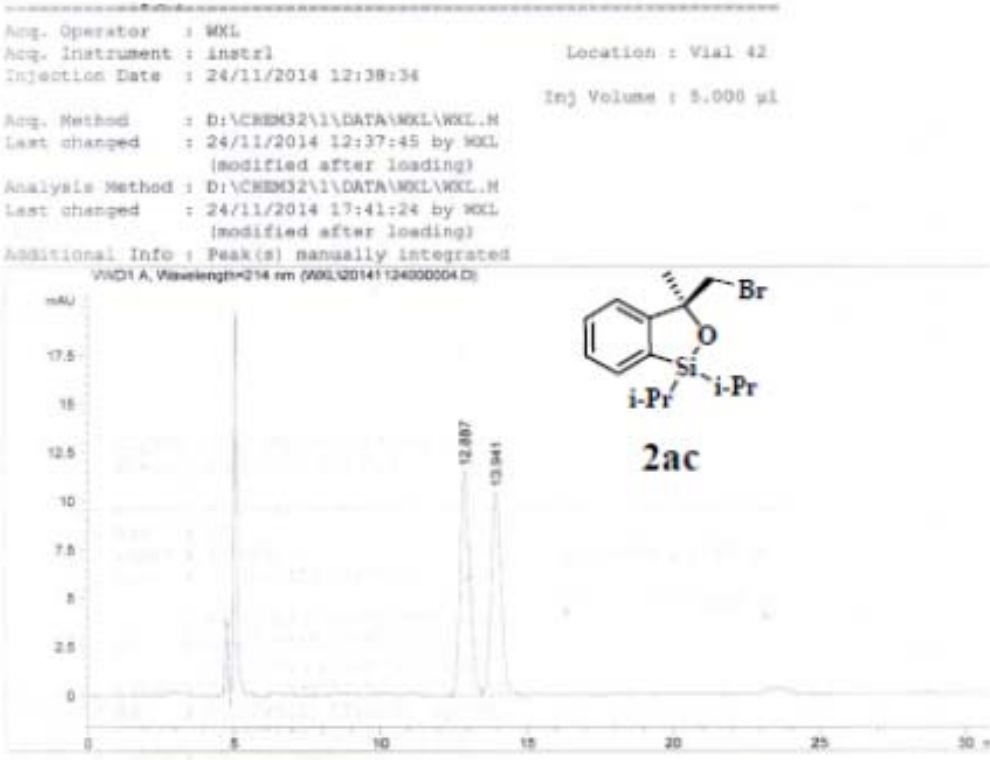

Ares pertent heport

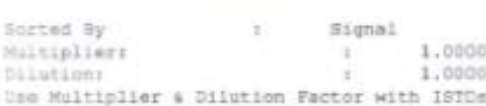

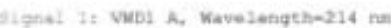

\begin{tabular}{|c|c|c|c|c|c|c|}
\hline thax & $\begin{array}{c}\text { Antrine } \\
\text { [nta] }\end{array}$ & 5yoe & $\begin{array}{l}\text { Width } \\
\text { [ain] }\end{array}$ & $\begin{array}{c}\text { Ares } \\
\text { [nation: }\end{array}$ & $\begin{array}{l}\text { Meight } \\
\text { [radi] }\end{array}$ & Ares: \\
\hline 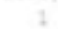 & & av & 0,3319 & 249.41347 & & 49,7258 \\
\hline$z$ & 13.961 & ve & 0.3716 & 231.16075 & 10.46150 & 50.2790 \\
\hline
\end{tabular}

w. End of Bapart ... 


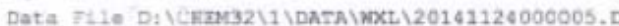
Sampl- Nane: X2T-2064-4B PC-3 M8W2 2140.7

Acg. Operator ; WXX.

Acq. Instrument i instr

Injection Date : 24/11/2014 13:11:24

Acq. Method : D: YCHEM32TIVDATAIWXLVWXL. M

Last changed $; 24 / 12 / 201413: 10 \div 06$ by kXi.

[nodified after loading)

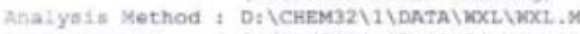

Last shanged : 24/11/2014 17:41:24 by WXL

(modified after loading!

hdditionel Info : Peak (s) manually Integrated

Location : Vial 43

In) Volume : 5,000

400

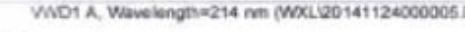

400

300

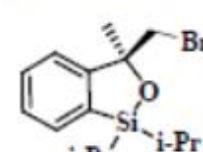

i-Pr ${ }^{\prime} \mathrm{i}-\mathrm{Pr}$

2 ac

200

100

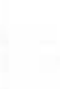

$\circ$

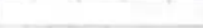

Area Percent Report

Sorted By

Haltiplier:

DSlution:

Signal

1.0000

1.0000
1.0000

Use Multiplier is Dilution Factor with ISTDs

signal It VWD1 h, Wavelengthm214 nn

\begin{tabular}{|c|c|c|c|c|c|c|}
\hline eak & $\begin{array}{c}\text { Retrime } \\
\text { [nin] }\end{array}$ & Type & $\begin{array}{l}\text { Width } \\
\text { [min] }\end{array}$ & $\begin{array}{c}\text { Area } \\
\text { [mAU*s] }\end{array}$ & $\begin{array}{l}\text { Height } \\
\text { (mAv) }\end{array}$ & $\begin{array}{c}\text { Area } \\
+\end{array}$ \\
\hline & & & & & & \\
\hline 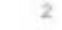 & 3.923 & wv & 0.3791 & 1876.35620 & 76.24662 & 15.9346 \\
\hline
\end{tabular}

7otals : 1.17753 ed 535.76696

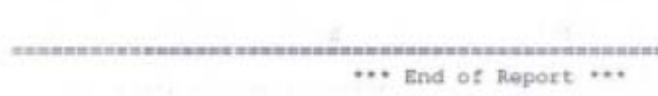

$\stackrel{20}{20}$

15 
Opeator.SC Timebase U3000 Sequence:WXL.2

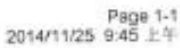

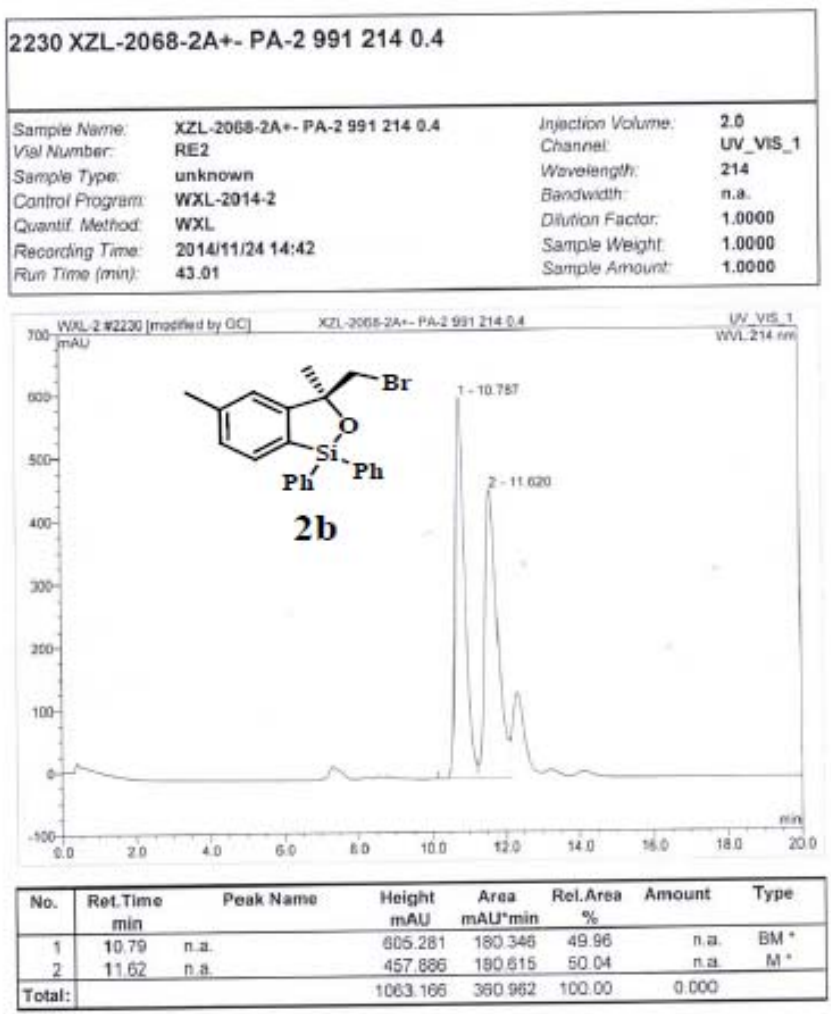




\begin{tabular}{|c|c|c|c|}
\hline \multicolumn{4}{|c|}{2231 XZL-2068-2B PA-2 9912140.4} \\
\hline Sample Name: & $X Z L-2068-28$ PA-2 9912140.4 & Injaction Volume: & 5.0 \\
\hline Vial Number: & RD3 & Channe: & UV_Vis_1 \\
\hline Sample Type: & unknown & Wavelengtt: & $214^{-}$ \\
\hline Control Arogram: & $W \times 2-2014-2$ & Bandwidin & n.a. \\
\hline Quantir Meihod: & $w \times L$ & Dilution Factor & 1.0000 \\
\hline Recording Time: & $2014 / 11 / 24$ 15:36 & Samplo Woight: & 1.0000 \\
\hline Run Trme (min): & 22.23 & Samale Amount: & 1.0000 \\
\hline
\end{tabular}

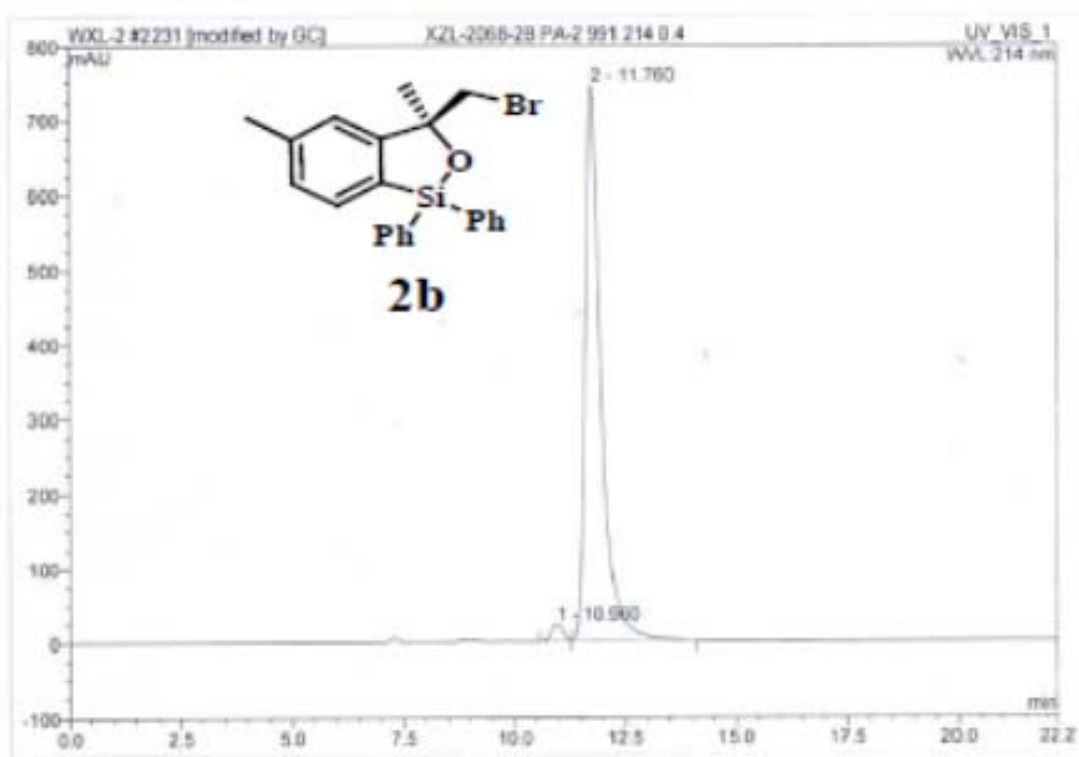

\begin{tabular}{|c|c|c|c|c|c|c|c|}
\hline No. & $\begin{array}{l}\text { Ret. Time } \\
\text { min }\end{array}$ & Peak Name & $\begin{array}{l}\text { Height } \\
\text { mAU }\end{array}$ & $\begin{array}{c}\text { Area } \\
\text { mAưmin }\end{array}$ & $\begin{array}{c}\text { Rel.Area } \\
\%\end{array}$ & Amount & Type \\
\hline 1 & 10.96 & $n a$ & 21.944 & 5969 & 1.88 & ก.a. & BNa \\
\hline 2 & 11.76 & n. & 742704 & 311364 & 98.12 & na. & BVB. \\
\hline Total: & & & 764648 & 317.332 & 100.00 & 0.000 & \\
\hline
\end{tabular}




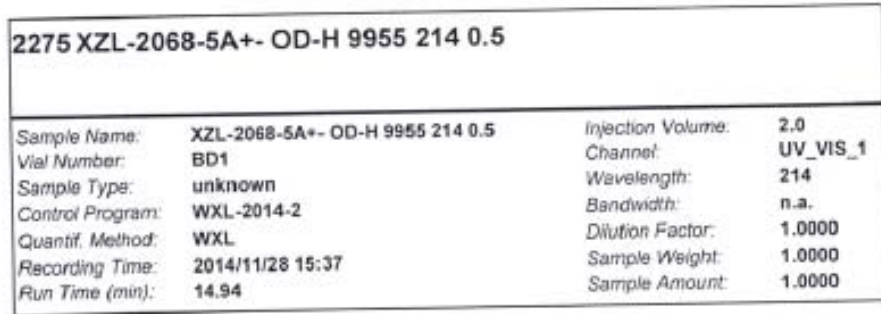

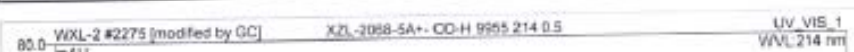

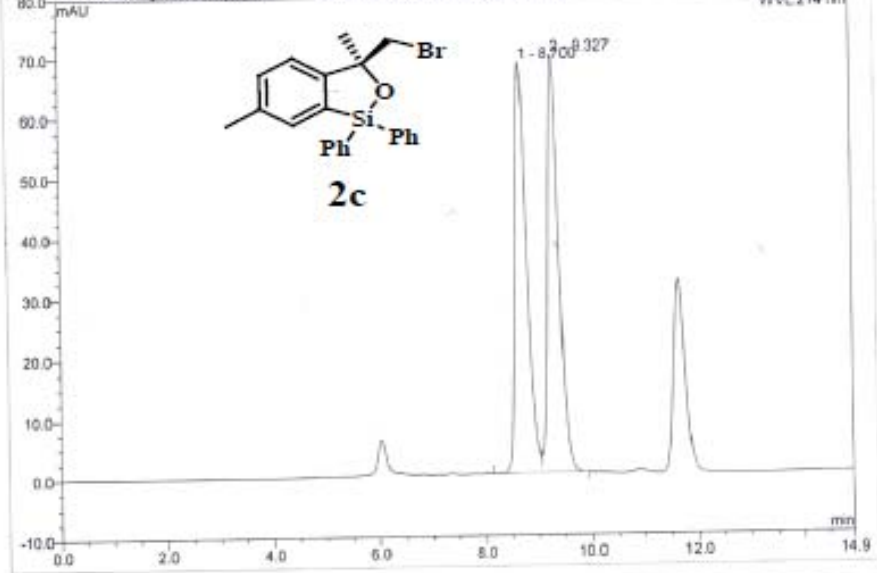

\begin{tabular}{|c|c|c|c|c|c|c|c|}
\hline No. & $\begin{array}{l}\text { Ret.Time } \\
\text { min }\end{array}$ & Peak Name & $\begin{array}{c}\text { Height } \\
\text { mAU }\end{array}$ & $\begin{array}{c}\text { Area } \\
\text { mAU'min }\end{array}$ & $\begin{array}{c}\text { Rel.Area } \\
\%\end{array}$ & Amount & Type \\
\hline $\begin{array}{l}1 \\
2\end{array}$ & 8.70 & n.a. & 68.304 & 16.774 & 49.83 & ก.8. & BM. \\
\hline$\frac{2}{\text { Total: }}$ & 9.33 & na. & 69.461 & $16.8 B 5$ & 50.17 & ก.a. & $\mathrm{MB}^{*}$ \\
\hline Total: & & & 137.766 & 33.658 & 100.00 & 0,000 & \\
\hline
\end{tabular}




\begin{tabular}{|llll|}
\hline 2276 XZL-2068-5B OD-H 99552140.5 & & \\
& & & \\
\hline Sample Name: & XZL-2060-5B OD-H 99552140.5 & injection Volume: & 2.0 \\
Vial Number: & BD2 & Channet: & UV_VIS_1 \\
Sample Type: & unknown & Wavolength: & 214 \\
Control Program: & WXL-2014-2 & Bandwidth: & n.a. \\
Quanti: Method: & WXL. & Oilution Factor & 1.0000 \\
Recording Time: & $2014 / 11 / 2815: 56$ & Sample Weight: & 1.0000 \\
Run Time (min): & 17.05 & Sample Amount: & 1.0000 \\
\hline
\end{tabular}

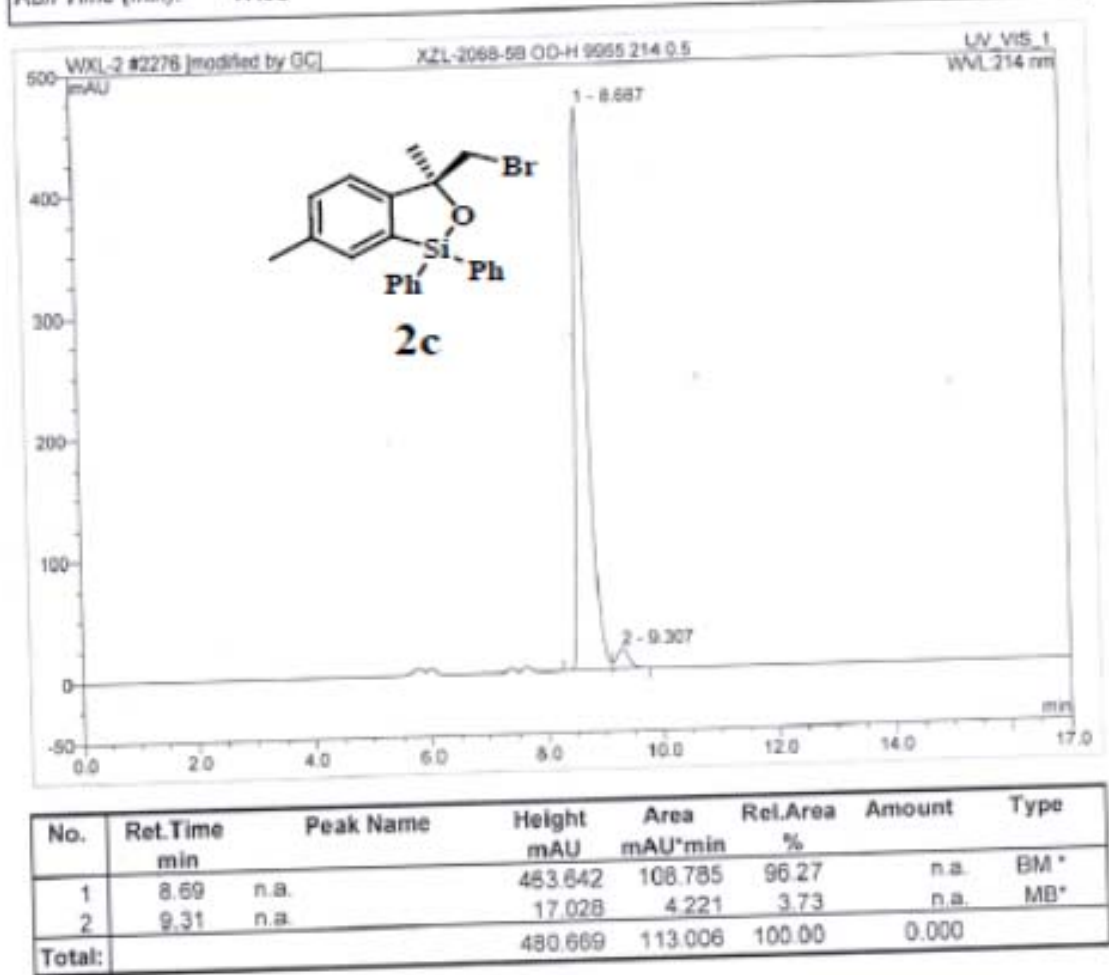


Operator.GC Timebase U3000 Sequence. WXL-2 Page 1-1
2014/11/25 g3at

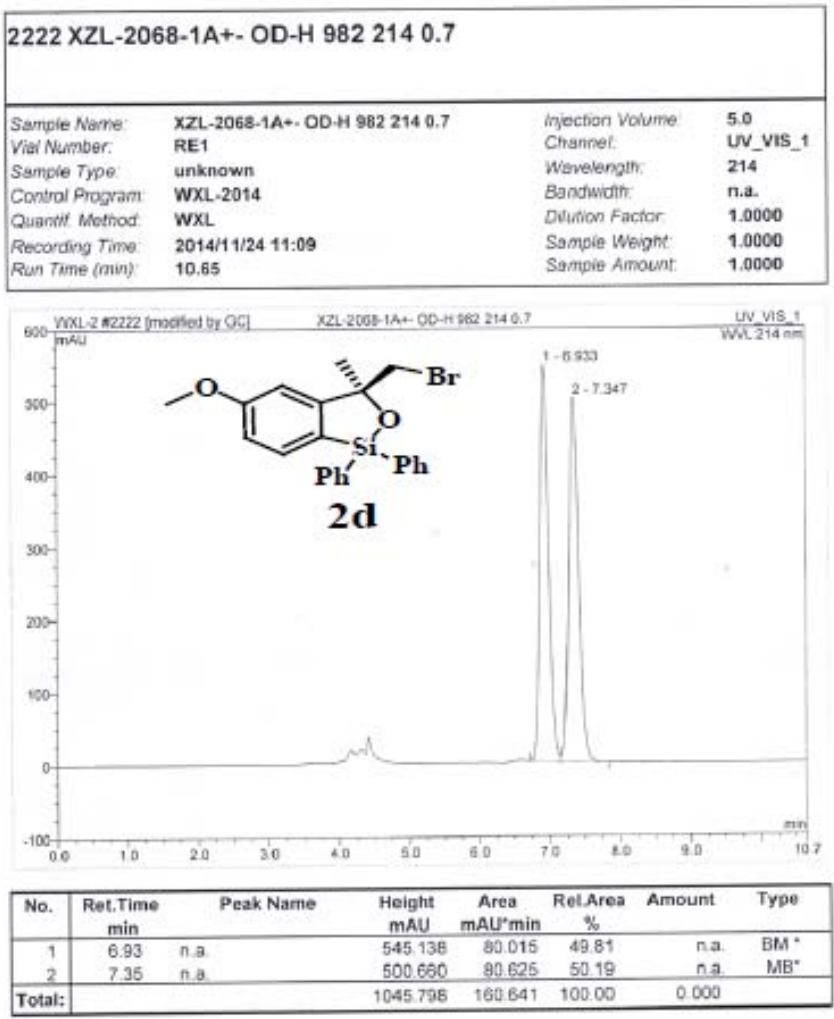




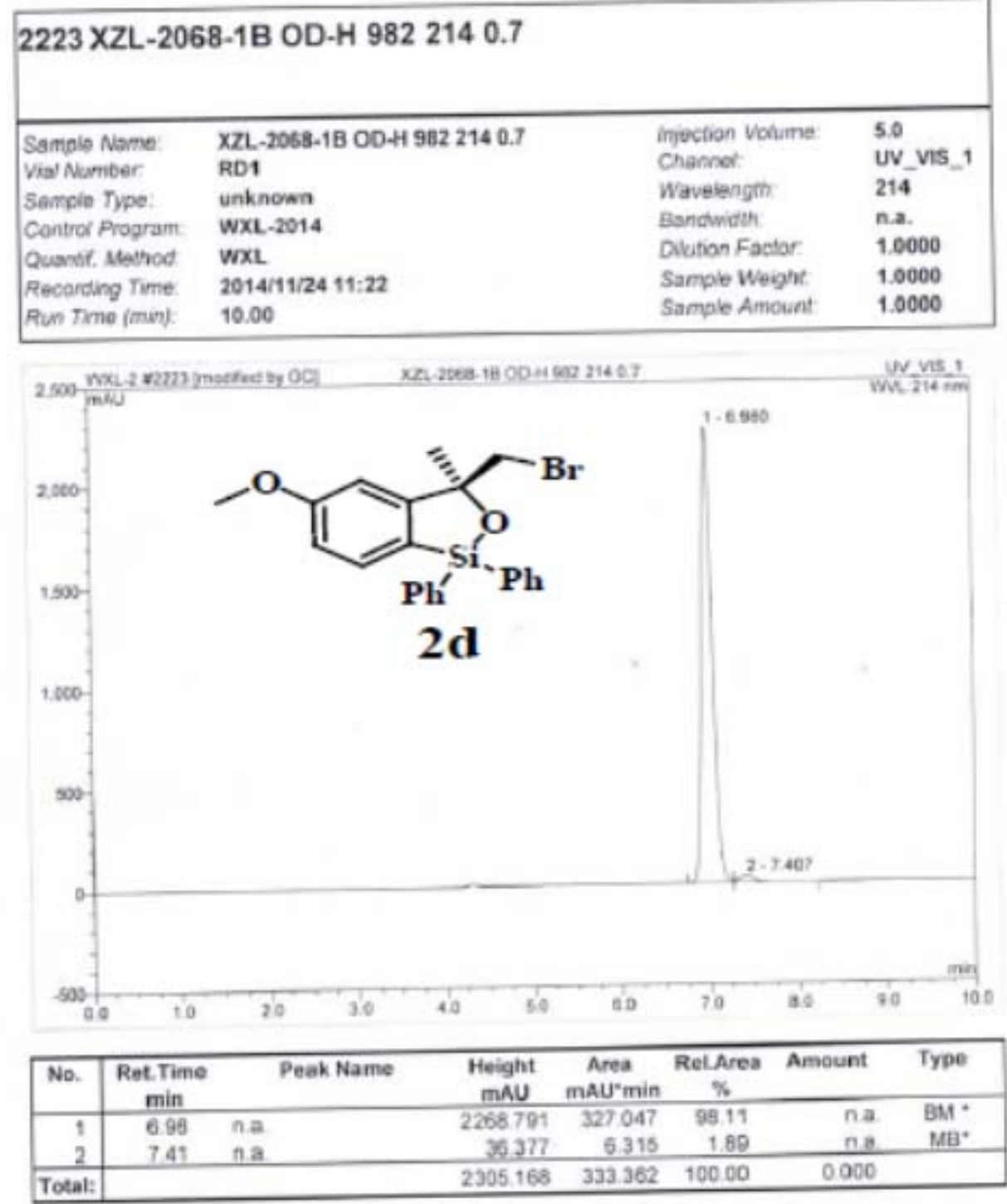




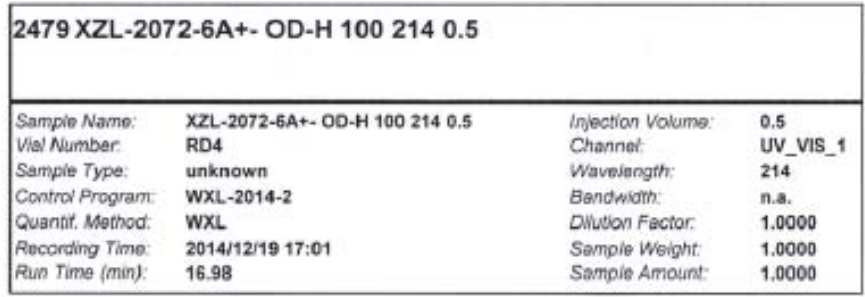

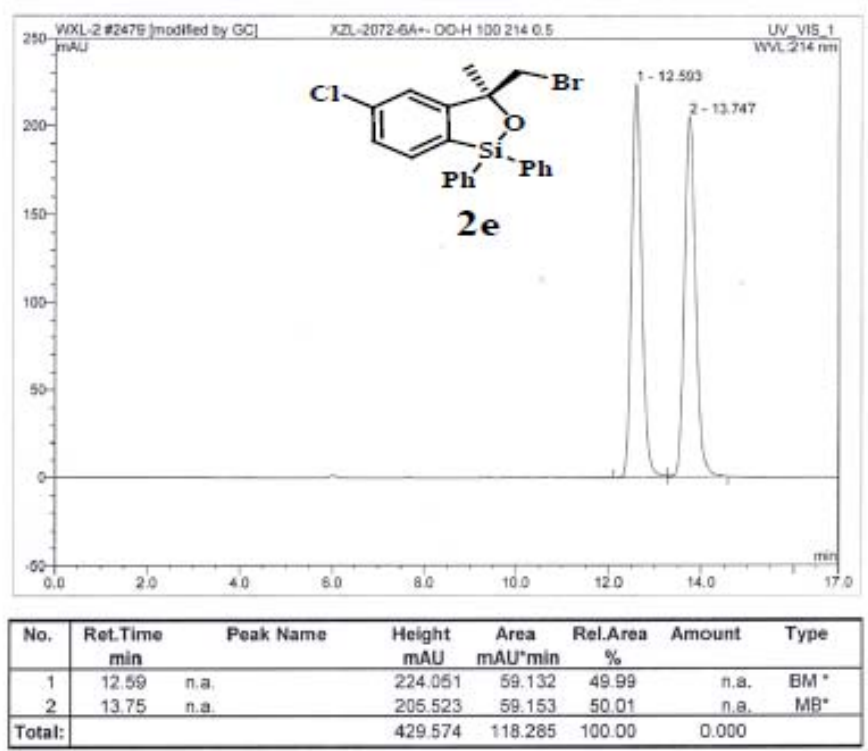




\section{XZL-2072-6B OD-H 1002140.5}

\begin{tabular}{|c|c|c|c|}
\hline Sample Name: & $X Z L-2072-68$ OD-H 1002140.5 & injection Volume: & 0.5 \\
\hline Vial Number: & RE4 & Channet & UV_Vis_1 \\
\hline Sample Type: & unknown & Weveiength: & 214 \\
\hline Control Program: & WXL-2014-2 & Bandwidth: & n.a. \\
\hline Quantil, Mothod: & WXL & Dilution Factor: & 1.0000 \\
\hline Recording Time: & $2014 / 12 / 1917: 40$ & Sampio Whight: & 1.0000 \\
\hline Run Time $(\min )$ : & 17.00 & Sample Amount & 1.0000 \\
\hline
\end{tabular}
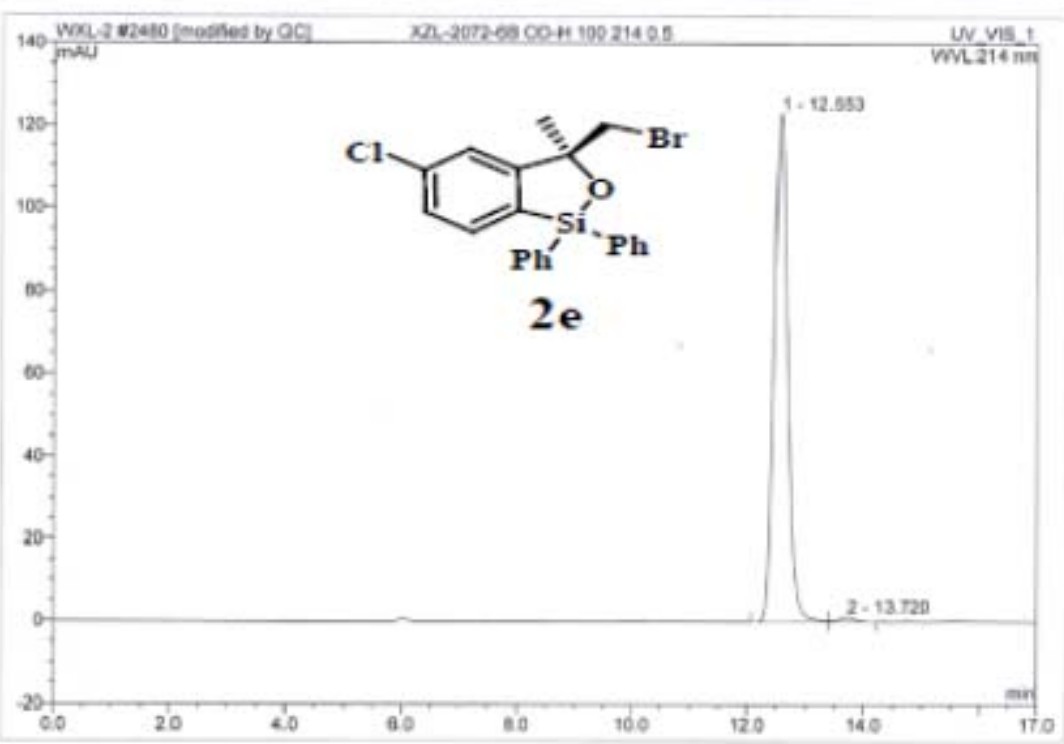

\begin{tabular}{|c|c|c|c|c|c|c|c|}
\hline No. & $\begin{array}{l}\text { Ret.Time } \\
\text { min }\end{array}$ & Peak Name & $\begin{array}{c}\text { Height } \\
\text { mAU }\end{array}$ & $\begin{array}{c}\text { Area } \\
\text { mAu*min }\end{array}$ & $\begin{array}{c}\text { Rel_Area } \\
\%\end{array}$ & Amount & Type \\
\hline 1 & 12.55 & n. 3 & 122.851 & 32.402 & 96.84 & na. & $B M^{*}$ \\
\hline 2 & 13.72 & $n a$ & 1.231 & 0.380 & 1.16 & na. & Ma' \\
\hline Total: & & & 124082 & 32.782 & 100.00 & 0.000 & \\
\hline
\end{tabular}




\begin{tabular}{|c|c|c|c|}
\hline \multicolumn{4}{|c|}{3870 XZL-2072-5A OD-H 1002140.7} \\
\hline $\begin{array}{l}\text { Sample Narme: } \\
\text { Viai Number: } \\
\text { Sample Type: } \\
\text { Contral Program: } \\
\text { Quantid. Mechod: } \\
\text { Recording Time: } \\
\text { Run Time (min): }\end{array}$ & $\begin{array}{l}\text { XZL-2072-5A OD-H } 1002140.7 \\
\text { RDS } \\
\text { unknown } \\
\text { test-dad } \\
\text { WXL } \\
2014-12-2722: 06 \\
45.00\end{array}$ & $\begin{array}{l}\text { injection Volume: } \\
\text { Channel: } \\
\text { Wavelength: } \\
\text { Bandwidth: } \\
\text { Ollution Fector: } \\
\text { Sample Weight: } \\
\text { Sample Amount: }\end{array}$ & $\begin{array}{l}1.0 \\
\text { UV_VIS_2 } \\
214.0 \\
4 \\
1.0000 \\
1.0000 \\
1.0000\end{array}$ \\
\hline
\end{tabular}

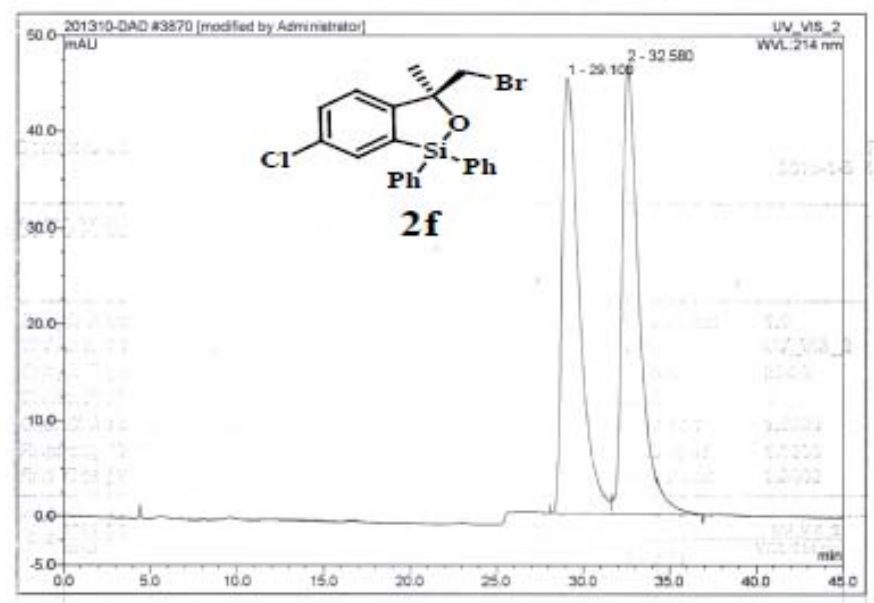

\begin{tabular}{|c|c|c|c|c|c|c|c|}
\hline No. & $\begin{array}{c}\text { Ret. Time } \\
\text { min }\end{array}$ & Peak Name & $\begin{array}{c}\text { Height } \\
\text { mAU }\end{array}$ & $\begin{array}{c}\text { Area } \\
\text { mAưmin }\end{array}$ & $\begin{array}{c}\text { Rel.Area } \\
\%\end{array}$ & Amount & Type \\
\hline 1 & 29.10 & ก.а. & 45.249 & 51.415 & 48.23 & ก.. & $8 M^{*}$ \\
\hline 2 & 32.58 & ก.a. & 46.754 & 55.193 & 51.77 & na & MB: \\
\hline Total: & & & 92.002 & 106.608 & 100.00 & 0.000 & \\
\hline
\end{tabular}




\begin{tabular}{|c|c|c|c|}
\hline \multicolumn{4}{|c|}{$3927 \times Z L-2072-5 B$ OD-H 1002140.7} \\
\hline Sample Name: & $X Z L-2072.5 B$ OD H 1002140.7 & injaction Voume: & 1.0 \\
\hline Vial Number: & RCS & Chamei: & UV_VIS_2 \\
\hline Sample Type: & unknown & Waveiengh: & 214.0 \\
\hline Control Program: & test-dad2 & Banowiden: & 4 \\
\hline Quannit Method: & wxL & Dilution Factor: & 1.0000 \\
\hline Recording Tine: & $2015-1-514: 22$ & Sample Woighe: & 1.0000 \\
\hline Run Time (min): & 42.37 & Sample Amount & 1.0000 \\
\hline
\end{tabular}

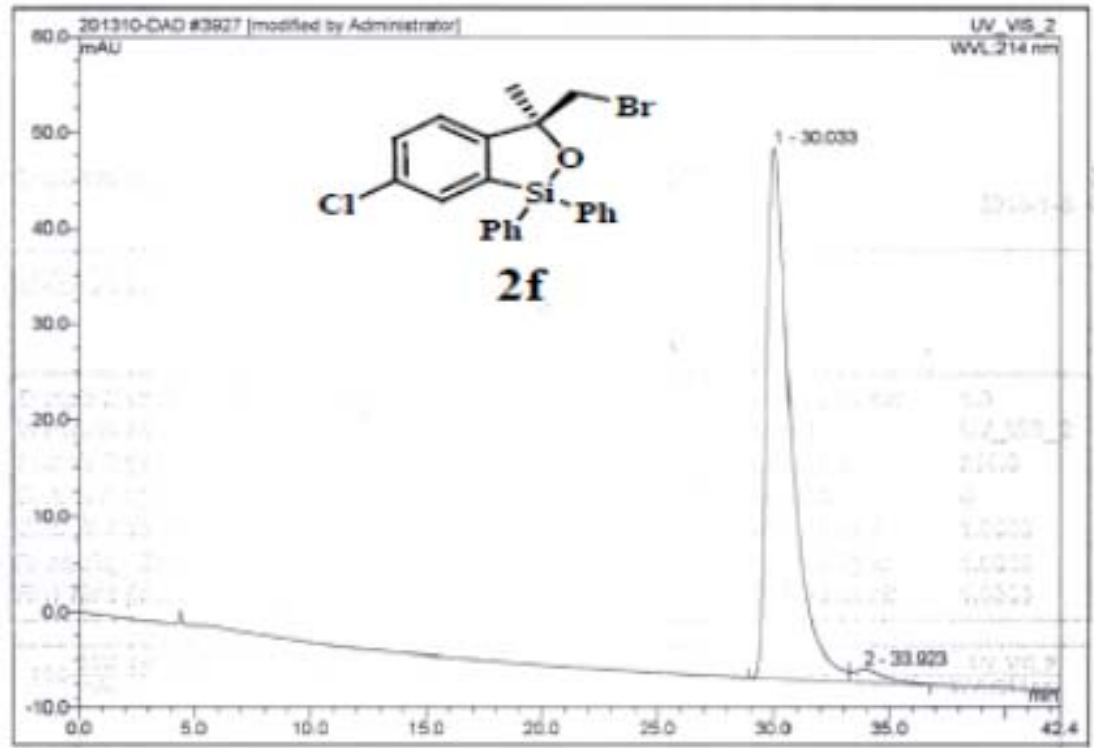

\begin{tabular}{|c|c|c|c|c|c|c|c|}
\hline No. & $\begin{array}{c}\text { Ret. Time } \\
\text { min }\end{array}$ & Peaik Name & $\begin{array}{l}\text { Height } \\
\text { mAU }\end{array}$ & $\begin{array}{c}\text { Arta } \\
\text { maurmin }\end{array}$ & $\begin{array}{c}\text { Rei_Area } \\
*\end{array}$ & Amount & Type \\
\hline 1 & 30.03 & n.a. & 55.357 & 67.486 & 07.01 & n.a. & BM. \\
\hline 2 & 33.92 & n.a. & 1368 & 2.070 & 2.99 & n.a. & $\mathrm{MB}^{*}$ \\
\hline Total: & & & 56.725 & 60.567 & 100.00 & 0.000 & \\
\hline
\end{tabular}




\begin{tabular}{|c|c|c|c|}
\hline \multicolumn{4}{|c|}{$2473 \times Z L-2072-4 A+-$ OD-H 1002140.5} \\
\hline $\begin{array}{l}\text { Sample Name: } \\
\text { Val Number: } \\
\text { Sample Type: } \\
\text { Control Program: } \\
\text { Quantif. Mothod: } \\
\text { Recording Time: } \\
\text { Run Time (min): }\end{array}$ & $\begin{array}{l}X Z L-2072-4 A+-O D-H 1002140.5 \\
\text { RD2 } \\
\text { unknown } \\
\text { WXL-2014-2 } \\
\text { WXL } \\
2014 / 1211916: 34 \\
25.45\end{array}$ & $\begin{array}{l}\text { Injectian Volume: } \\
\text { Channel: } \\
\text { Wavalength: } \\
\text { Bandwidth: } \\
\text { Dhution Factor: } \\
\text { Sample Weight: } \\
\text { Sample Amount: }\end{array}$ & $\begin{array}{l}0.5 \\
\text { UV_VIS_1 } \\
214 \\
\text { n.a. } \\
1.0000 \\
1.0000 \\
1.0000\end{array}$ \\
\hline
\end{tabular}

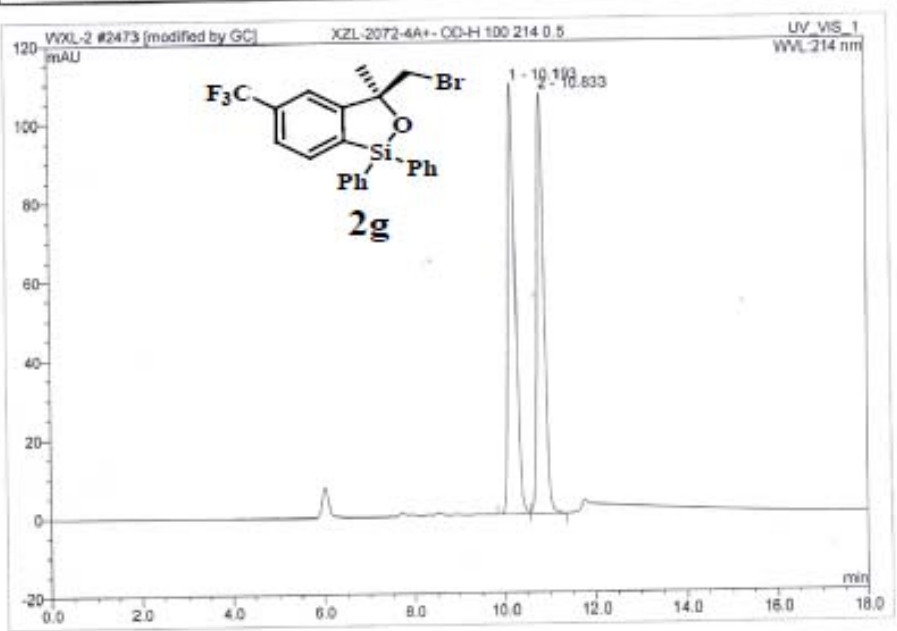

\begin{tabular}{|c|c|c|c|c|c|c|c|}
\hline No. & $\begin{array}{c}\text { Ret.Time } \\
\text { min }\end{array}$ & Peak Name & $\begin{array}{l}\text { Height } \\
\text { mAU }\end{array}$ & $\begin{array}{c}\text { Area } \\
\text { mAU'min }\end{array}$ & $\begin{array}{c}\text { Rel.Aroa } \\
\%\end{array}$ & Amount & Type \\
\hline 1 & 10.19 & n.a. & 109.586 & 19.712 & 49.77 & n.a. & $B M^{\circ}$ \\
\hline 2 & 10.83 & n.a. & 107.314 & 19.897 & 50.23 & na & M. \\
\hline Total: & & & 216.860 & 39.609 & 100.00 & 0.000 & \\
\hline
\end{tabular}




\section{XZL-2072-4B OD-H 1002140.5}

\begin{tabular}{|c|c|c|c|}
\hline Sampie Name: & $X Z L-2072-4 B$ OD-H 1002140.5 & Injection Volume: & 0.5 \\
\hline Vial Number: & RE3 & Channet: & UV_VIS_1 \\
\hline Sample Type: & unknown & Wavelength: & 214 \\
\hline Control Program: & $W X L-2014-2$ & Bandwiath: & n.a. \\
\hline Quantif, Mothod: & WXL & Dilution Factor: & 1.0000 \\
\hline Recording Time: & $2014 / 12 / 19$ 17:21 & Sample Weight: & 1.0000 \\
\hline Run Time (min): & 17.00 & Samale Amount: & 1.0000 \\
\hline
\end{tabular}

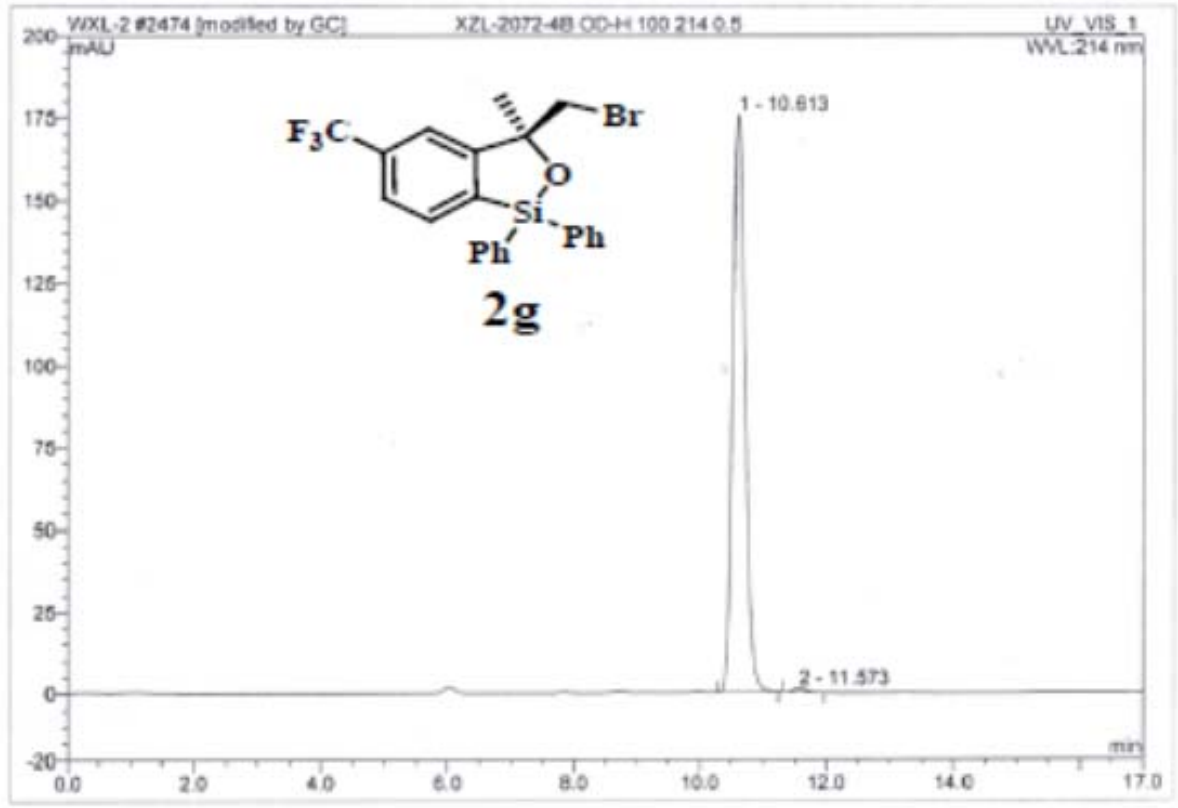

\begin{tabular}{|c|c|c|c|c|c|c|c|}
\hline No. & $\begin{array}{c}\text { Ret.Time } \\
\text { min }\end{array}$ & Peak Name & $\begin{array}{c}\text { Height } \\
\text { mAU }\end{array}$ & $\begin{array}{c}\text { Area } \\
\text { mAU'min }\end{array}$ & $\begin{array}{c}\text { Rel.Area } \\
\%\end{array}$ & Amount & Type \\
\hline 1 & 10.61 & n.a. & 175.428 & 37.406 & 99.38 & n.a. & BMA \\
\hline 2 & 11.57 & $\mathrm{na}$ & 1.087 & 0.233 & 0.62 & n. 3. & $B M B^{\circ}$ \\
\hline Total: & & & 176.496 & 37.639 & 100.00 & 0.000 & \\
\hline
\end{tabular}


Operator.GC Timebsse:U3000 Sequence:WXL-2 $\begin{array}{rl}\text { Page } 1-1 \\ 2014 / 12 / 1 & g 30 \text { 土. }\end{array}$

$2271 \times Z L-2068-3 A+-$ OD-H 1002140.5

\begin{tabular}{|c|c|c|c|}
\hline $\begin{array}{l}\text { Sazinple Name: } \\
\text { Vial Number: }\end{array}$ & $\begin{array}{l}X Z L \text {-2068-3A+- OD-H } 1002140.5 \\
\text { BC1 }\end{array}$ & $\begin{array}{l}\text { Inpoction Volume: } \\
\text { Charnet: }\end{array}$ & $\begin{array}{l}2.0 \\
\text { UV_vis_1 }\end{array}$ \\
\hline $\begin{array}{l}\text { Vial Mumber: } \\
\text { Sampie Type: }\end{array}$ & $\begin{array}{l}\text { BC1 } \\
\text { unknown }\end{array}$ & Wavelength. & 214 \\
\hline Control Program: & WXL-2014-2 & Bandwidth: & n.a. \\
\hline Quantit. Method. & $w \times L$ & Dilution Factor: & 1.0000 \\
\hline Recarding Tins: & $2014 / 11 / 28$ 16:44 & Sample Weight: & 1.0000 \\
\hline RuAn Time (min): & 26.39 & Sampie Amount: & 1.0000 \\
\hline
\end{tabular}

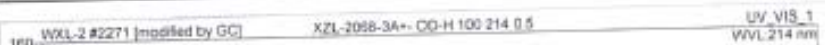

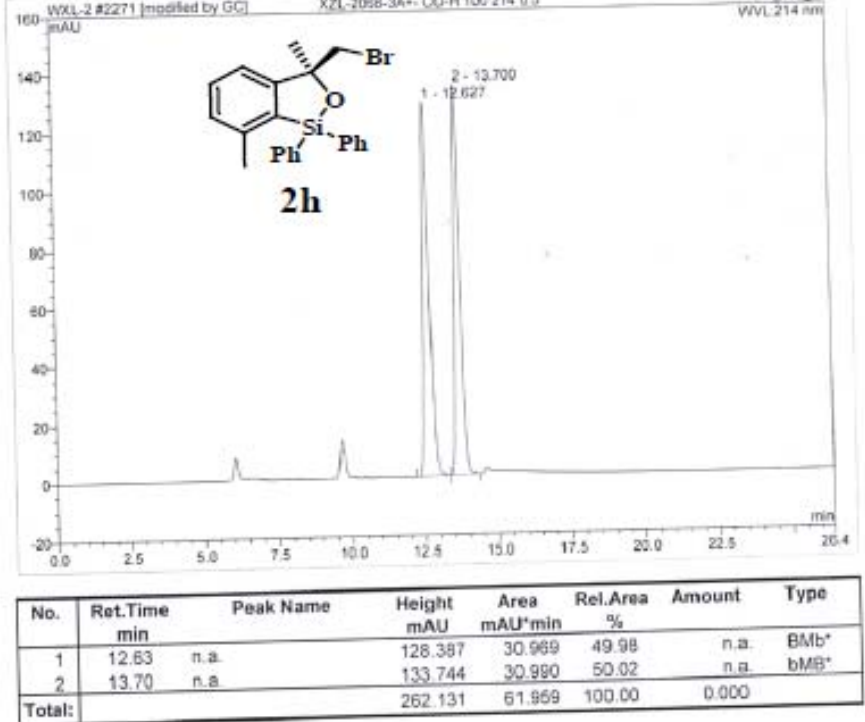




\begin{tabular}{|c|c|c|c|}
\hline \multicolumn{4}{|c|}{2272 XZL-2068-3B OD-H 1002140.5} \\
\hline $\begin{array}{l}\text { Sample Name: } \\
\text { Vial Number: }\end{array}$ & $\begin{array}{l}X Z L-2068-3 B O D-H 1002140.5 \\
B C 2\end{array}$ & $\begin{array}{l}\text { Injoction Volume: } \\
\text { Channet. }\end{array}$ & $\begin{array}{l}2.0 \\
\text { UV_VIS_1 }\end{array}$ \\
\hline Sample Type: & unknown & Wavelength: & 214 \\
\hline Contral Program: & $W \times L-2014-2$ & Bandwiath: & n.a. \\
\hline Quanst. Method: & wXL & Dilution Factor & 1.0000 \\
\hline Recoording Time: & 2014/11/28 17:12 & Sampie Weight & 1.0000 \\
\hline Run Time (min): & 28.49 & Samplo Anount: & 1.0000 \\
\hline
\end{tabular}
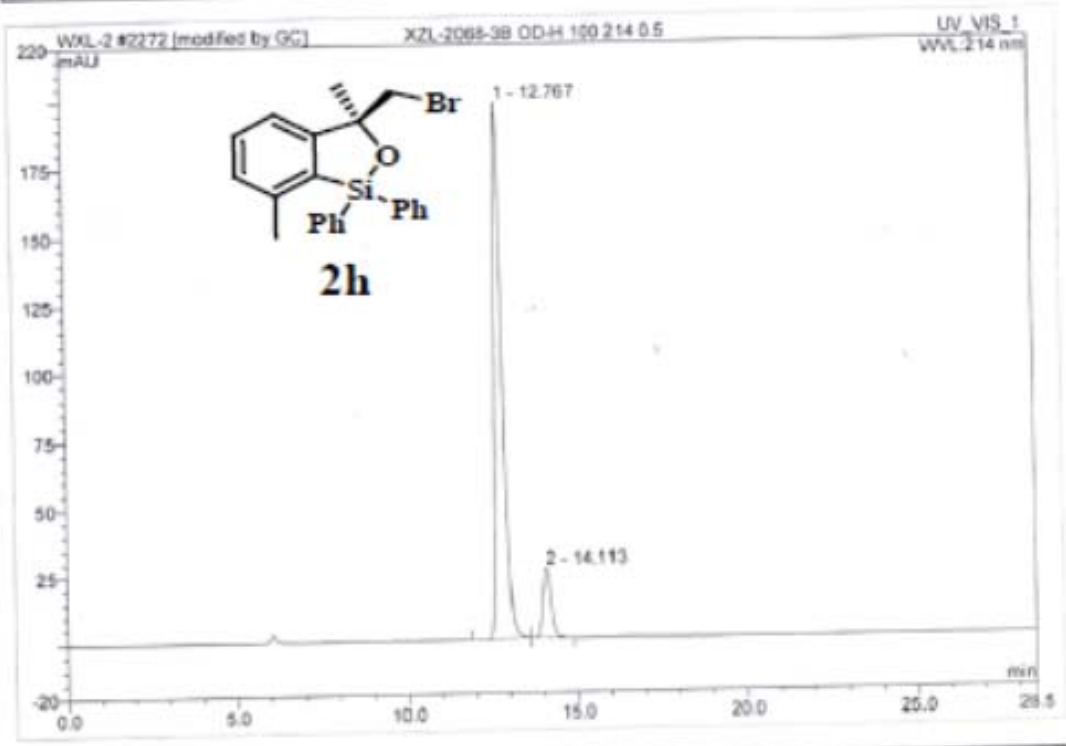

\begin{tabular}{|c|c|c|c|c|c|c|c|}
\hline No. & $\begin{array}{l}\text { Ret.Time } \\
\text { min }\end{array}$ & Peak Name & $\begin{array}{l}\text { Height } \\
\text { maU }\end{array}$ & $\begin{array}{c}\text { Aroa } \\
\text { mAU'min }\end{array}$ & $\begin{array}{c}\text { Rel.Area } \\
\%\end{array}$ & Amount & Type \\
\hline $\begin{array}{l}1 \\
2\end{array}$ & 12.77 & n. $\mathbf{a}$ & $\begin{array}{r}198.136 \\
26.033\end{array}$ & $\begin{array}{r}53.480 \\
7.428 \\
\end{array}$ & $\begin{array}{l}87.80 \\
12.20\end{array}$ & $\begin{array}{l}\text { n.a. } \\
\text { n.a. }\end{array}$ & $\begin{array}{l}\text { BMA* } \\
\text { BMB* }\end{array}$ \\
\hline$\frac{2}{\text { Total: }}$ & 14.11 & na & 224169 & 60.908 & 100.00 & 0,000 & \\
\hline
\end{tabular}




\begin{tabular}{|c|c|c|c|}
\hline \multicolumn{4}{|c|}{2349 XZL-2071-4A+- OD-H 9912140.7} \\
\hline $\begin{array}{l}\text { Sampie Name: } \\
\text { Viaj Number: } \\
\text { Sampie Type: } \\
\text { Control Program: } \\
\text { Quantr., Mothod: } \\
\text { Recorting Time: } \\
\text { Run Timo (min): }\end{array}$ & $\begin{array}{l}\text { XZL-2071-4A+-OD-H } 9912140.7 \\
\text { BB3 } \\
\text { unknown } \\
\text { WXL-2014-2 } \\
\text { WXL } \\
2014 / 121815: 41 \\
14.51\end{array}$ & $\begin{array}{l}\text { Injection Valume: } \\
\text { Channet: } \\
\text { Wavelength: } \\
\text { Bandwidth: } \\
\text { Dulution Foctor: } \\
\text { Sample Weight } \\
\text { Samplo Amount: }\end{array}$ & $\begin{array}{l}2.0 \\
\text { UV_VIS_1 } \\
214 \\
\text { n.a. } \\
1.0000 \\
1.0000 \\
1.0000\end{array}$ \\
\hline
\end{tabular}

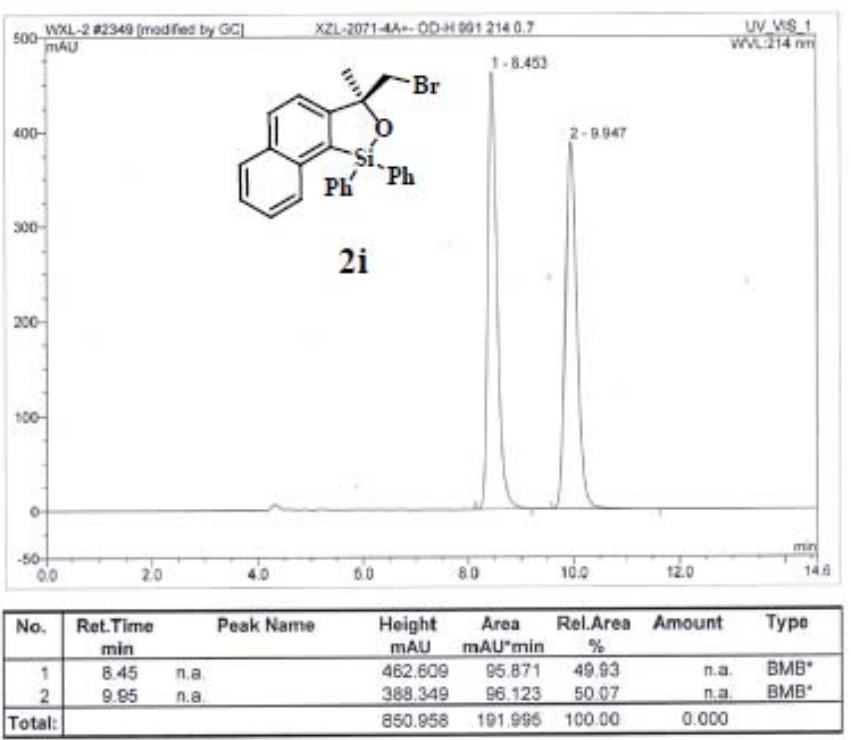




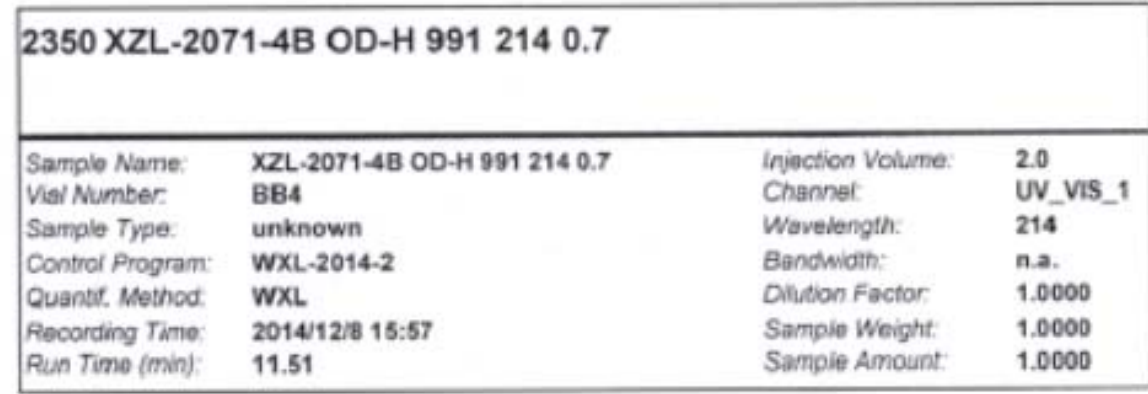

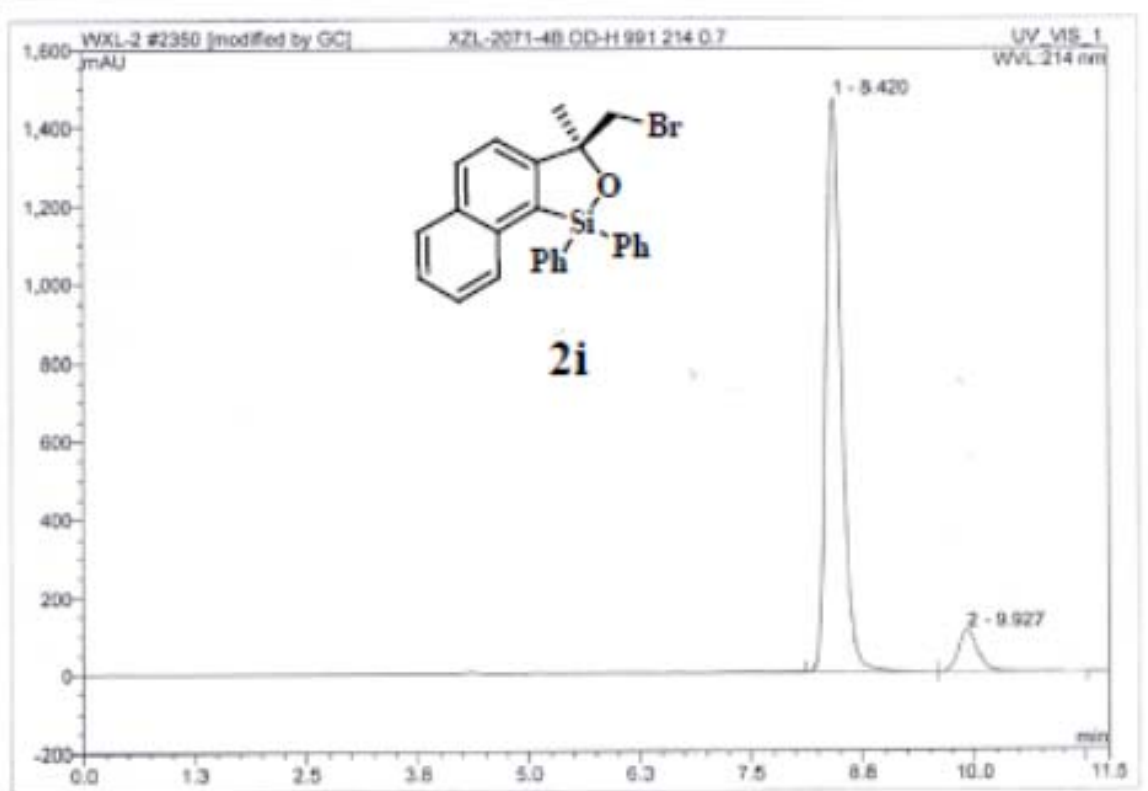

\begin{tabular}{|c|c|c|c|c|c|c|c|}
\hline No. & $\begin{array}{c}\text { Ret. Time } \\
\text { min }\end{array}$ & Peak Name & $\begin{array}{c}\text { Height } \\
\text { mAU }\end{array}$ & $\begin{array}{c}\text { Area } \\
\text { maU'min }\end{array}$ & $\begin{array}{c}\text { Rel.Area } \\
\% \\
\end{array}$ & Amount & Type \\
\hline 1 & 8.42 & n.a. & 1470.423 & 303.541 & 91.96 & n.a. & $8 M^{\circ}$ \\
\hline 2 & 9.93 & n.a. & 105.618 & 26524 & 8.04 & na & $1 / \mathrm{B}^{*}$ \\
\hline Total: & & & 1576.041 & 330.006 & 100.00 & 0.000 & \\
\hline
\end{tabular}




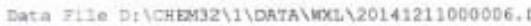
Samplo Name: X2L-2072-1A+- PC-3 M9K1 $224 \quad 0.720$

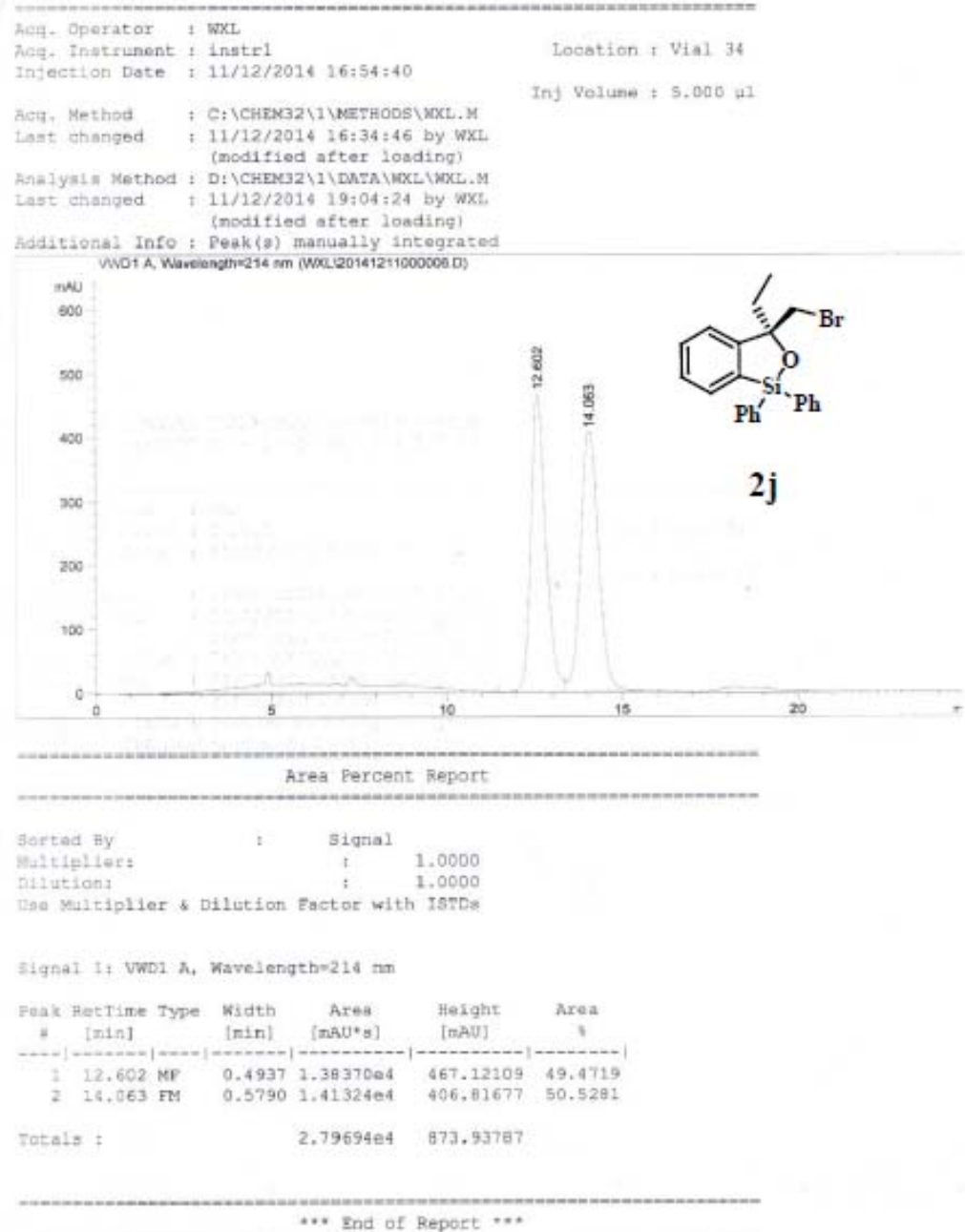

... End of Report *... 


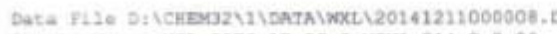
5arple Name: X2L-2072-18 PC-3 N9w1 214 D.7 20

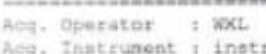

thiection Date : $11 / 12 / 2014 \quad 17: 57: 2$ ?

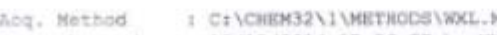

Last ohanged $\quad+11 / 12 / 2014$ 17:56:37 by wxe

(modified after leading)

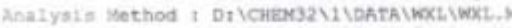

Lant ahanged $1,11 / 12 / 201419: 04+24$ by wX:

(nodified after loading)

Adationai Info + Peak(a) nanually integrated

$\operatorname{mos}$

320

450

300

200

200

100

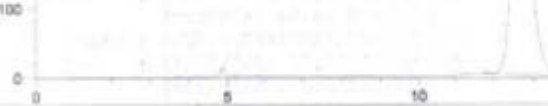

Area Percent Heport

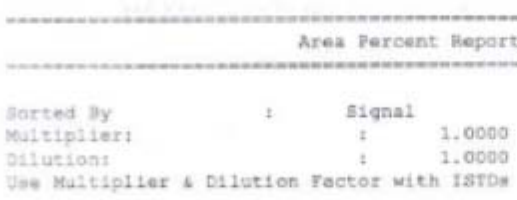

Hgha1 if vuDi $\mathrm{A}$, wavelength-214 ni

\begin{tabular}{|c|c|c|c|c|c|c|}
\hline Fesk & $\begin{array}{c}\text { Aotrine } \\
\text { [nia] }\end{array}$ & Type & $\begin{array}{l}\text { Widtb } \\
\text { [nin] }\end{array}$ & $\begin{array}{c}\text { Area } \\
{[\operatorname{maD} * s]}\end{array}$ & $\begin{array}{l}\text { Height } \\
\text { [nAU] }\end{array}$ & $\begin{array}{c}\text { Aren } \\
1\end{array}$ \\
\hline & & & 18 & 2.51 & & 93.7992 \\
\hline 2 & 14.188 & $y_{b}$ & 0,5572 & 998.86963 & 27.27710 & 6.2006 \\
\hline
\end{tabular}

rotals

$1.6304604 \quad 531.1698 \%$
Location : Vial 35

Inj Volune $: 2.000 \mathrm{p}$

8

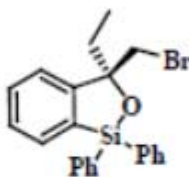

$2 \mathrm{j}$

*.. and of Report +.. 


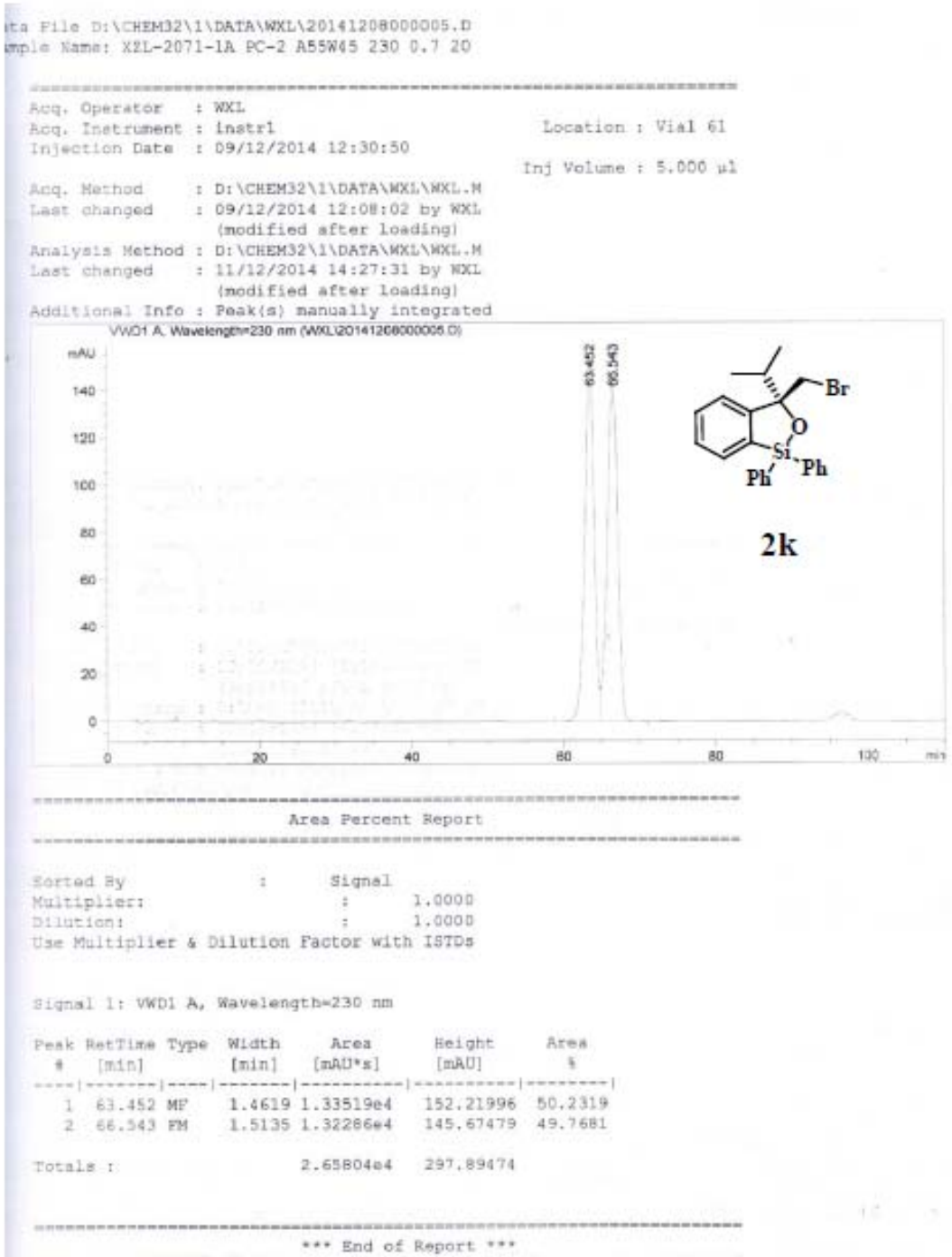




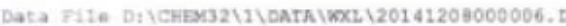

Sample Name: XzL-2071-1日 PC-2 A55W45 $230 \quad 0.728$

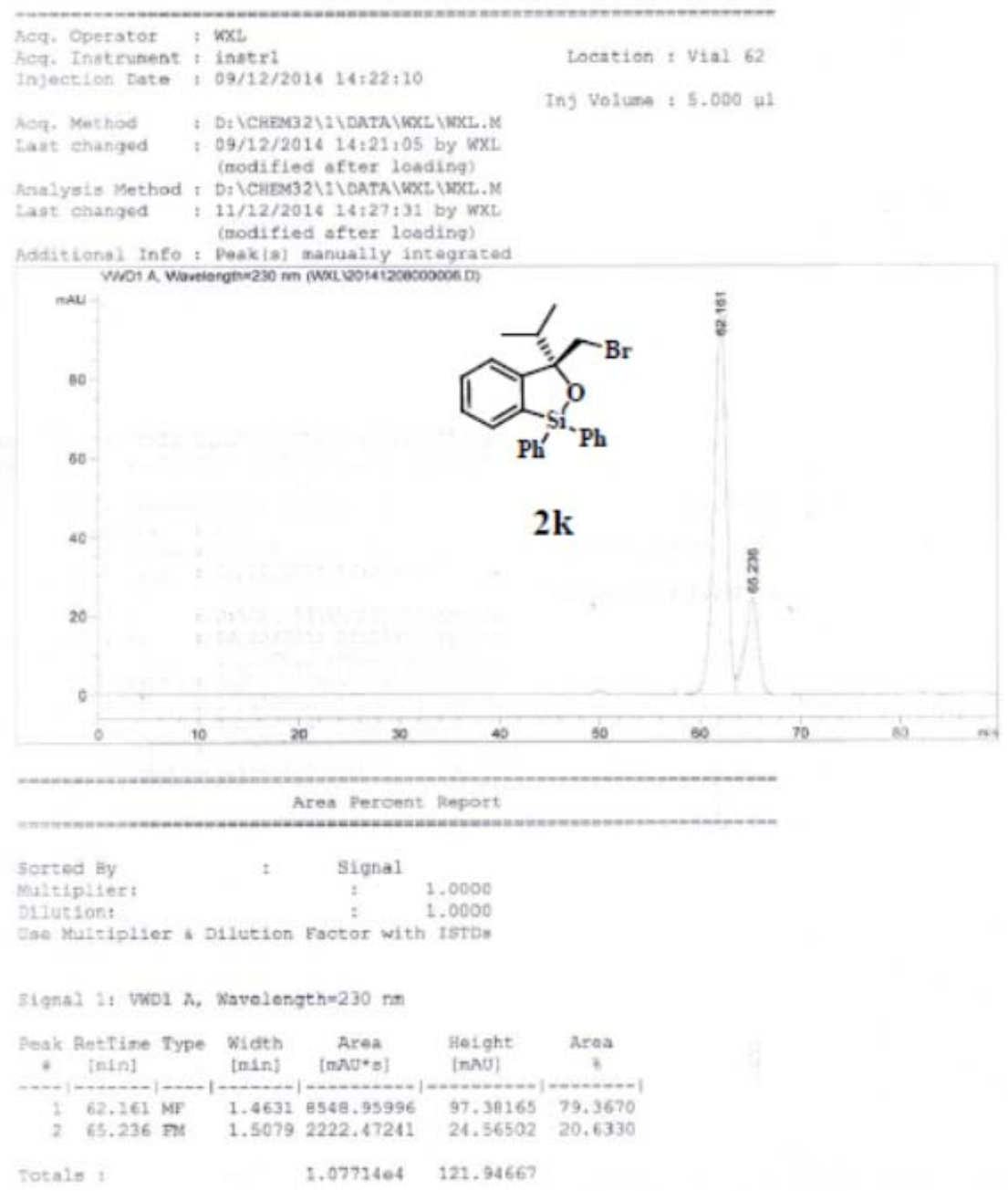

*.. End of Report * 


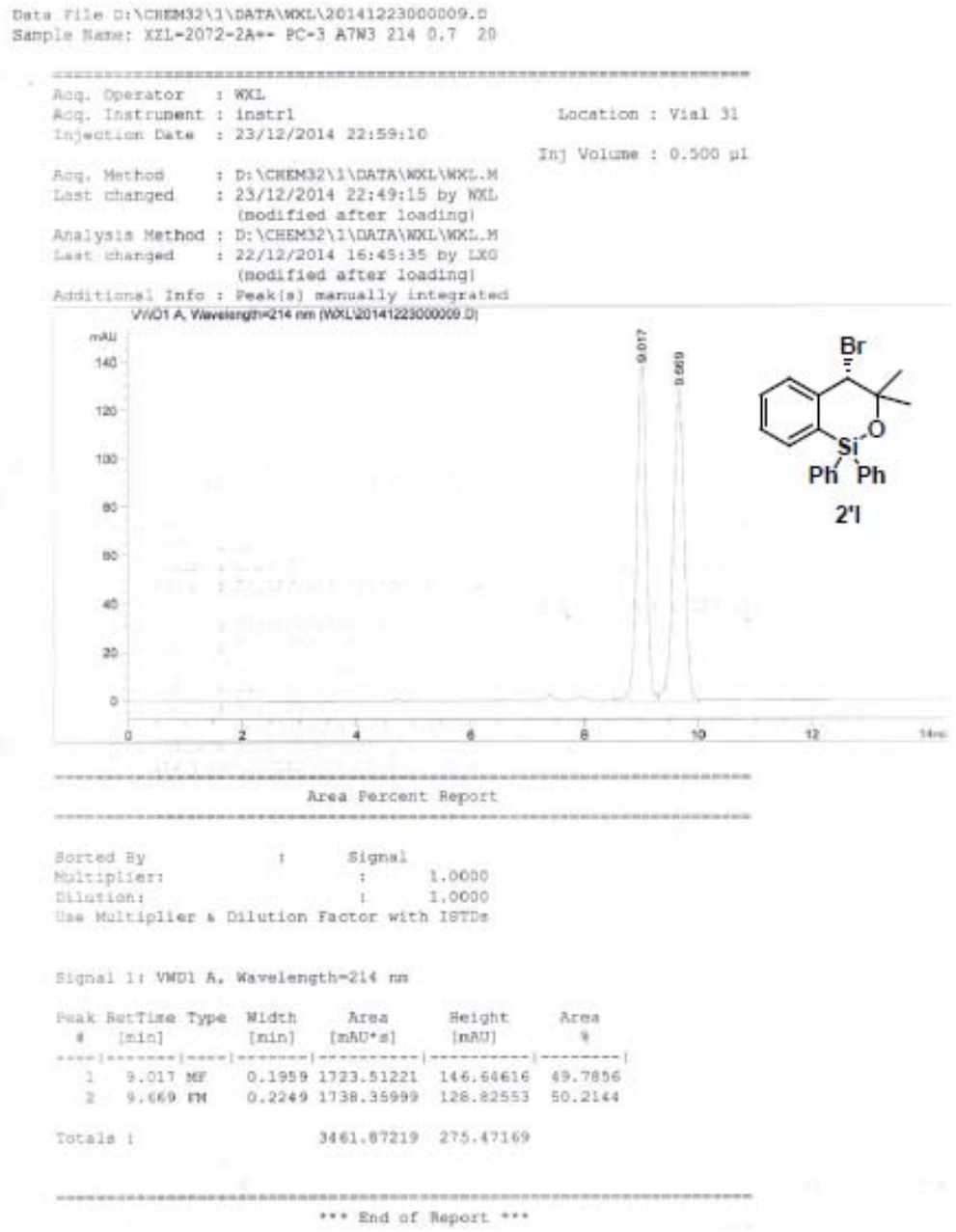




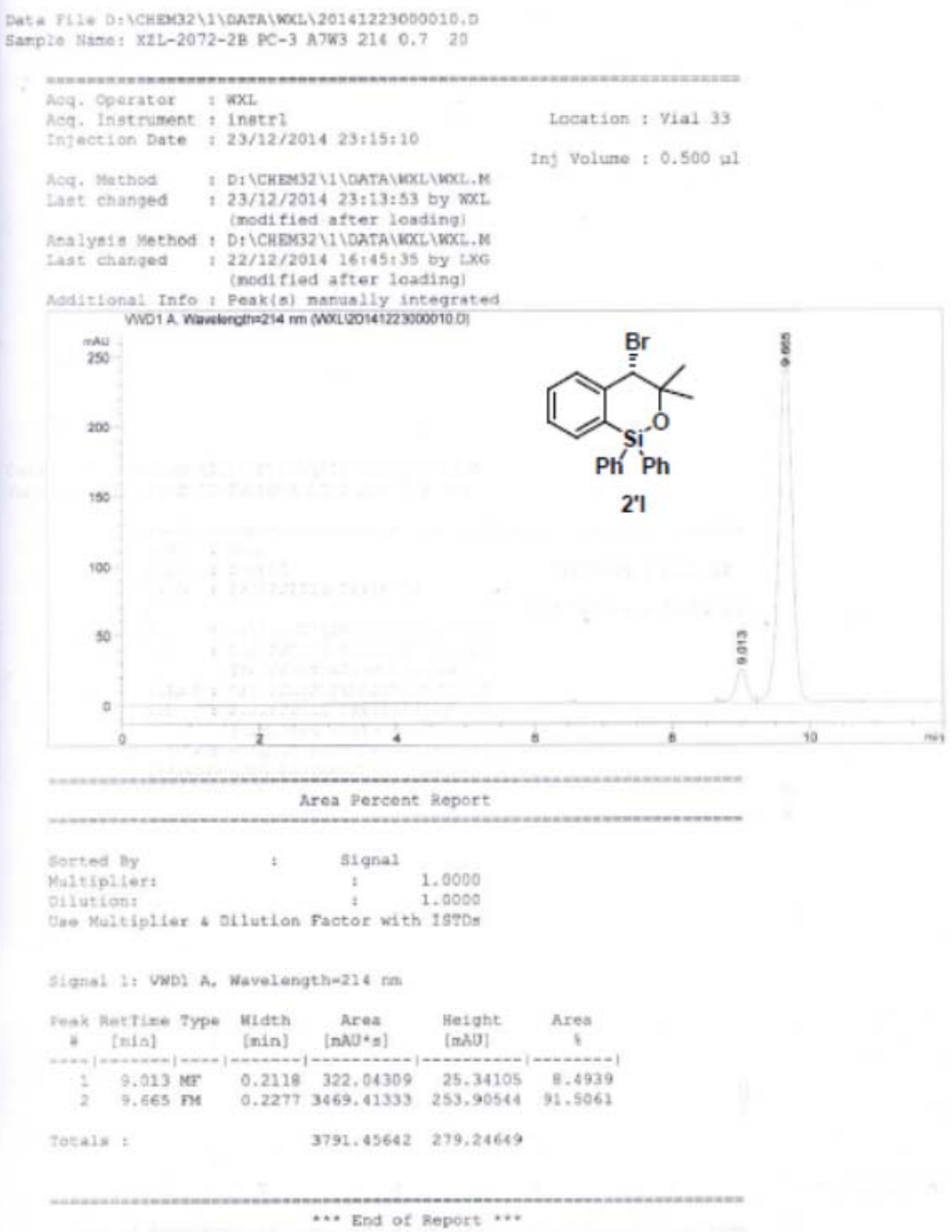




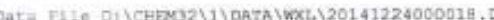

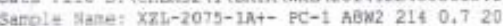

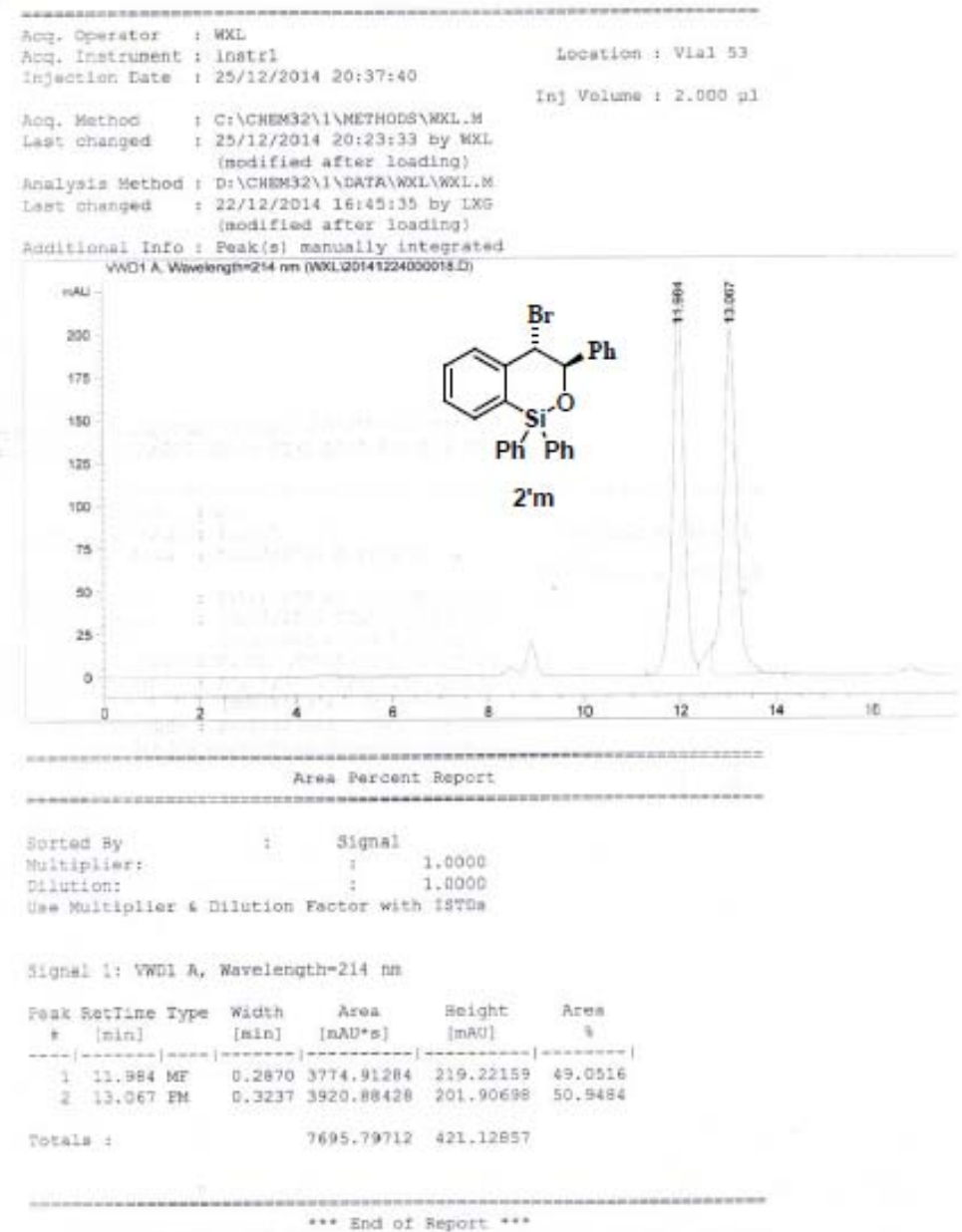

... Fnd of Rerort ...' 


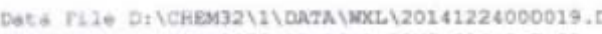
Sanple Nane: XZL-2075-1B PC-1 A81K2 2160.720

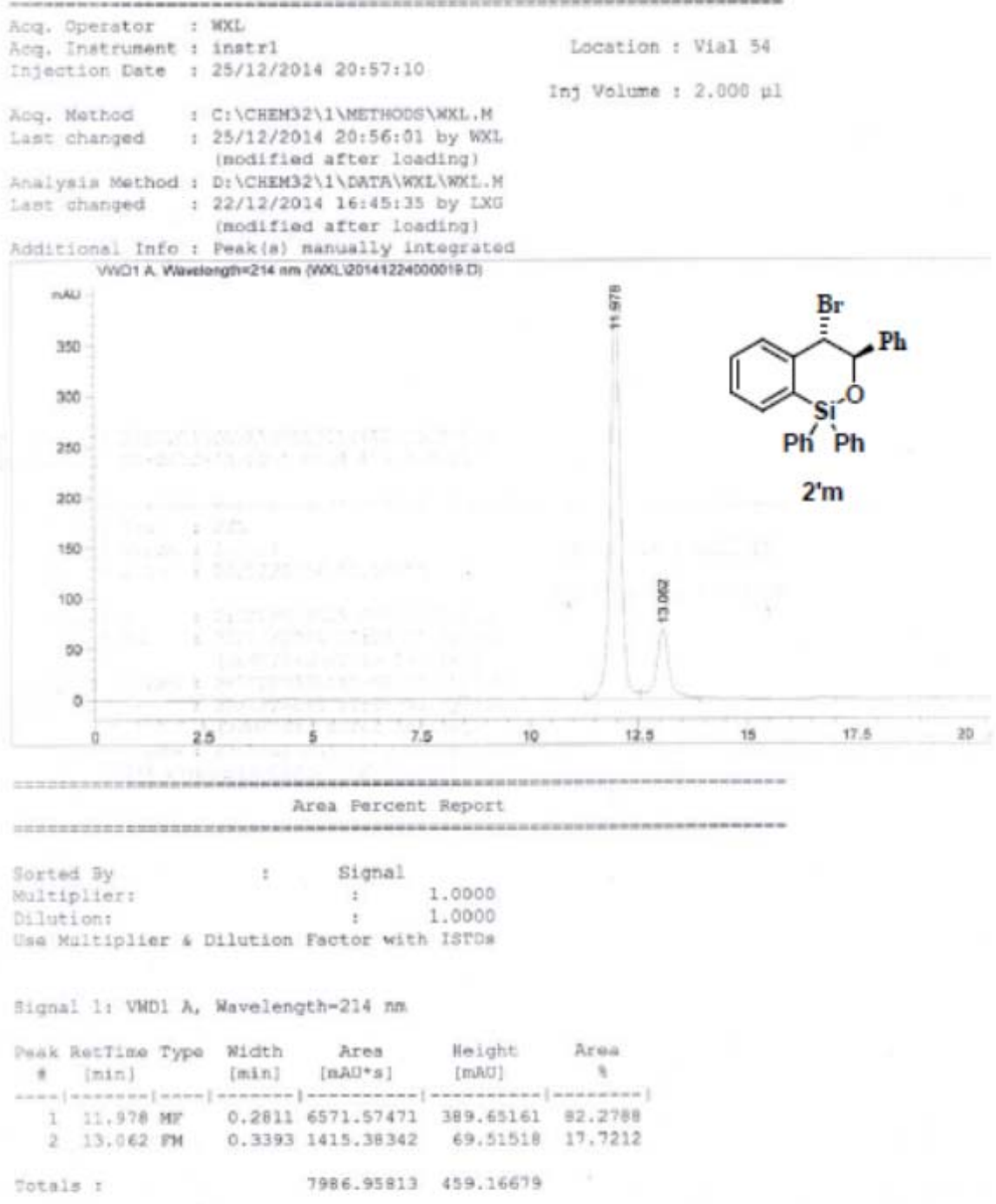

... End of Report ... 


\begin{tabular}{|c|c|c|c|}
\hline \multicolumn{4}{|c|}{4085 XZL-2075-5A+- AD-H 9912140.7} \\
\hline $\begin{array}{l}\text { Sample Name: } \\
\text { Vial Number: }\end{array}$ & $\begin{array}{l}\text { XZL-2075-SA+-AD-H } 9912140.7 \\
\text { RD1 }\end{array}$ & $\begin{array}{l}\text { Mjection Vocurne: } \\
\text { Chamear }\end{array}$ & $\begin{array}{l}3.0 \\
\text { UV_Vis_2 }\end{array}$ \\
\hline Sample Type: & unknown & Wavaiangth: & 214.0 \\
\hline Control Program: & test-dad & Banowiven: & 4 \\
\hline Quantr. Mothod: & $w \times L$ & Dutution Factor: & 1.0000 \\
\hline Recouthy Time: & $2016-1 \cdot-26$ 16:16 & Sampre Woight & 1.0000 \\
\hline Run Time (min): & 13.00 & Sampite Amount: & 1.0000 \\
\hline
\end{tabular}

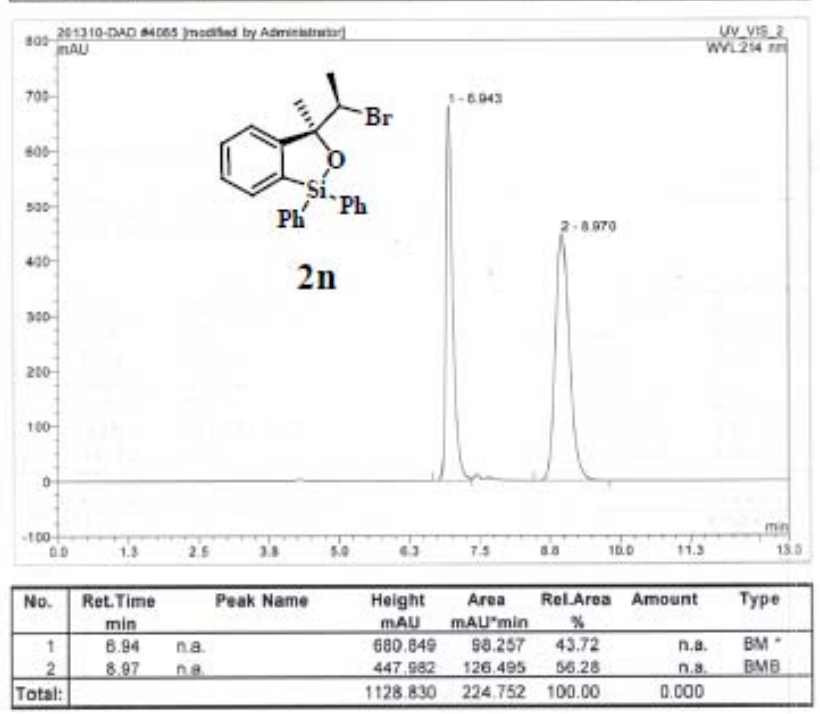




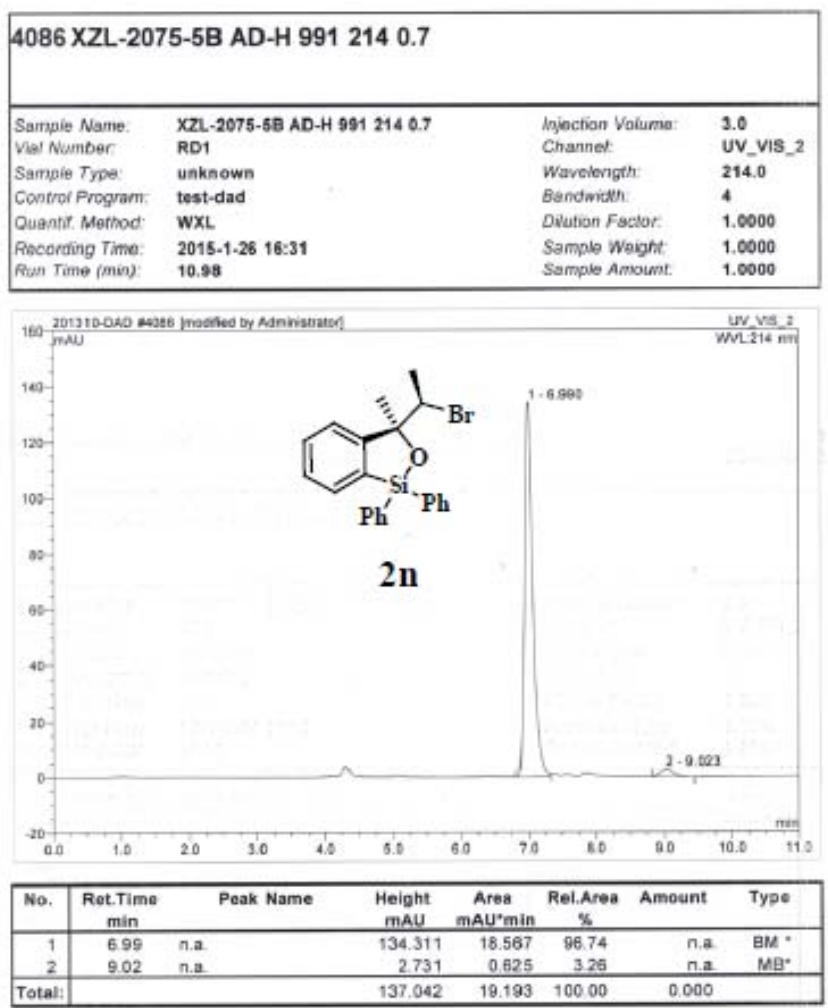




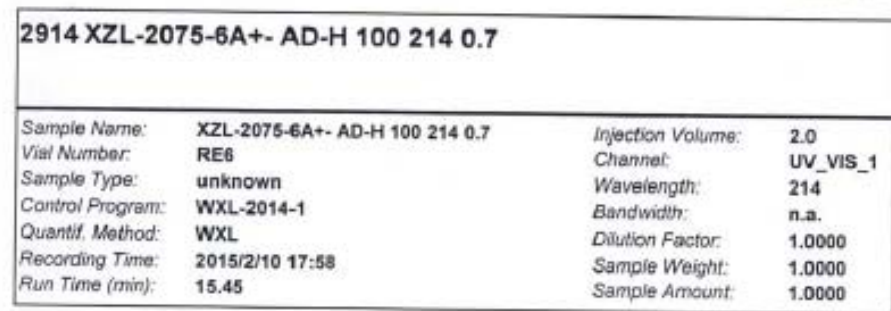

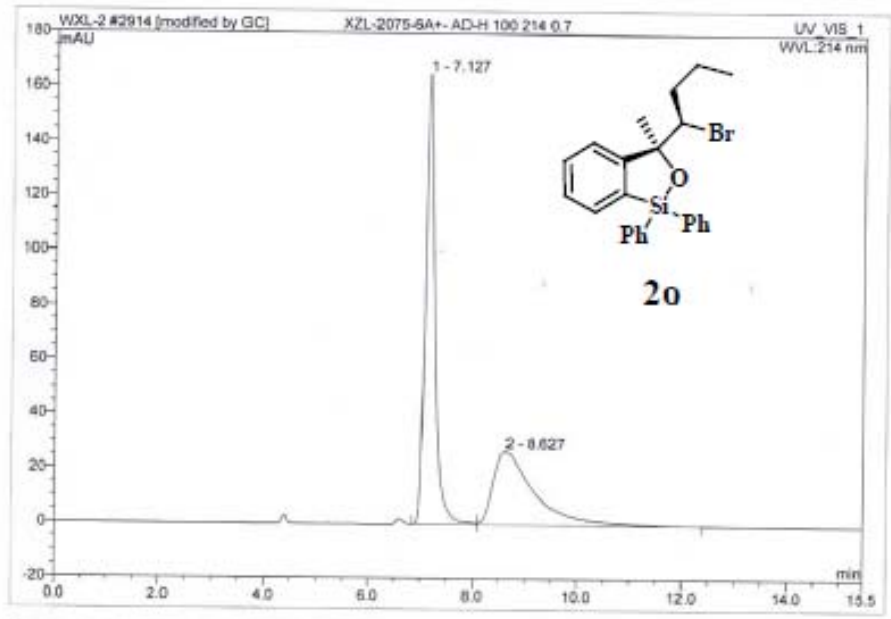

\begin{tabular}{|c|c|c|c|c|c|c|c|}
\hline No. & $\begin{array}{c}\text { Ret.Time } \\
\text { min }\end{array}$ & Peak Name & $\begin{array}{c}\text { Height } \\
\text { mAU }\end{array}$ & $\begin{array}{c}\text { Area } \\
\text { mAU'min }\end{array}$ & $\begin{array}{c}\text { Rel.Area } \\
\%\end{array}$ & Amount & Type \\
\hline 1 & 7,13 & n.a. & 165.028 & 35.852 & 58.96 & ก. . & $8 M^{\circ}$ \\
\hline 2 & 8.63 & n. & 27.009 & 24955 & 41.04 & n. & $\mathrm{M}^{*}$ \\
\hline Total: & & & 192.039 & 60.807 & 100.00 & 0.000 & \\
\hline
\end{tabular}




\begin{tabular}{|c|c|c|c|}
\hline \multicolumn{4}{|c|}{2915 XZL-2075-6B AD-H 1002140.7} \\
\hline $\begin{array}{l}\text { Sample Name: } \\
\text { Vial Numbiar: }\end{array}$ & $\begin{array}{l}\text { XZL-2075-6B AD-H } 1002140.7 \\
\text { RE7 }\end{array}$ & $\begin{array}{l}\text { hyection Volume: } \\
\text { Channel: }\end{array}$ & 2.0 \\
\hline Sample Type: & unknown & Waveiongth: & 214 \\
\hline Control Program: & $W X L-2014-1$ & Bandwioth: & n.a. \\
\hline Quantil. Method: & WXL & Divution Factor: & 1.0000 \\
\hline Rocording Time: & 2015/2/10 18:18 & Sample Weight: & 1.0000 \\
\hline Run Time (min): & 21.69 & Sample Amount: & 1.0000 \\
\hline
\end{tabular}

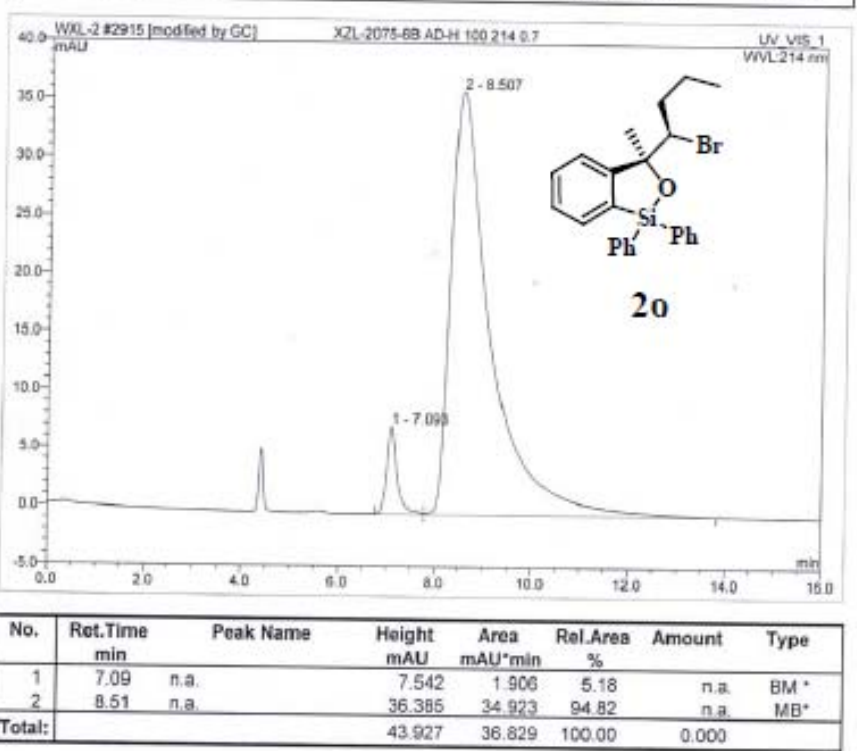




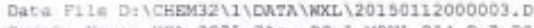
Sampla Nama: XZL-2075-3At- PC-1 M9N1 $214 \quad 0.720$

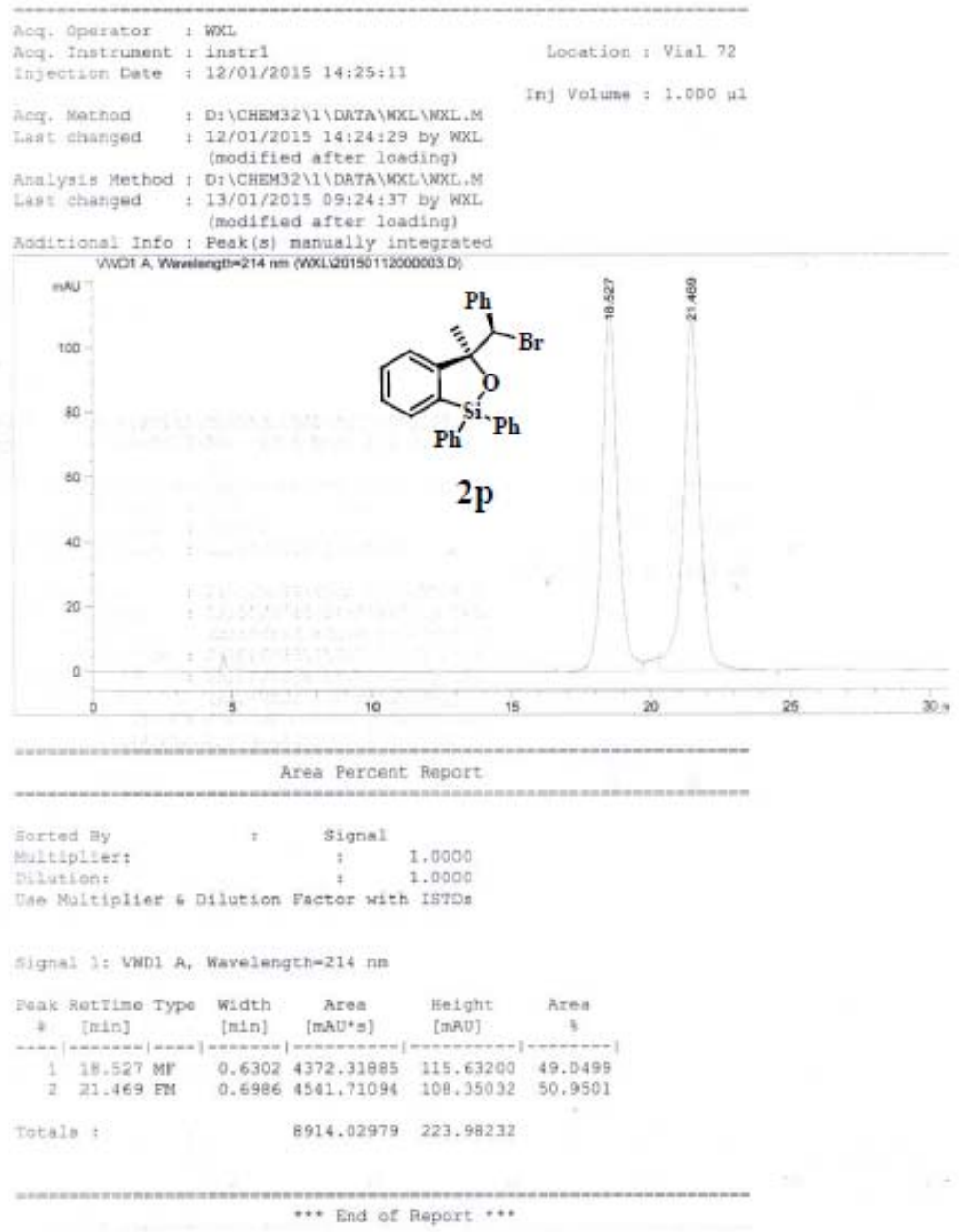

$\cdots$ End of Report $\cdots$ 


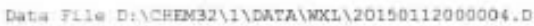
5arpla Name: XZL-2075-3A PC-1 M961 $214 \quad 0.720$

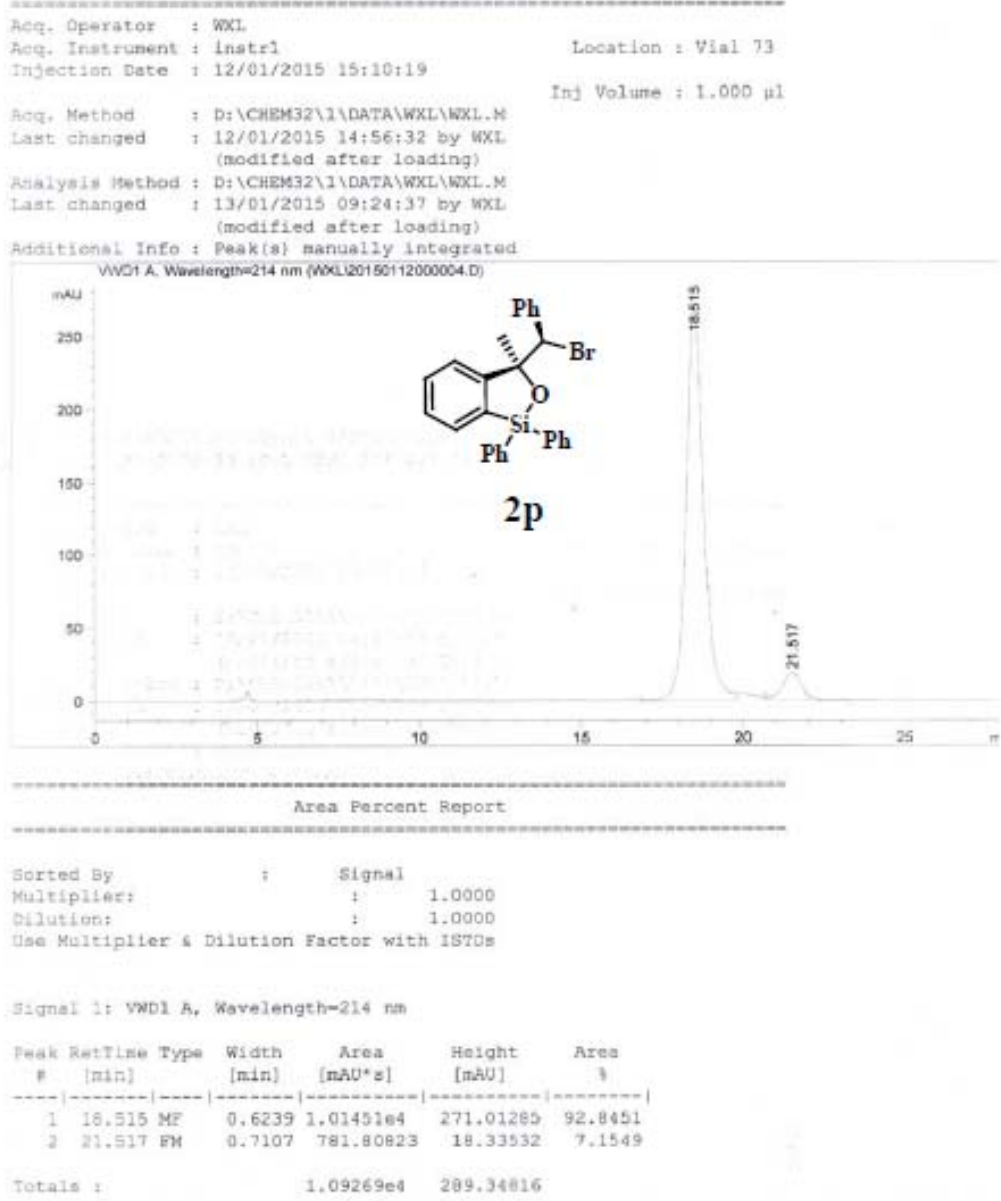

Location : Vial 73

ing Vol une $\neq 1.000 \mathrm{H}$

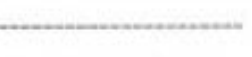

100
000




\begin{tabular}{|llll|}
\hline 2349 XZL-2071-5A+- AD-H 9912140.7 & & \\
& & \\
\hline Sample Name: & XZL-2071-5A+- AD-H 9912140.7 & injection Volume: & 2.0 \\
Vial Number: & BB1 & Channet: & UV_VIS_1 \\
Sample Type: & unknown & Wavelength: & 214 \\
Cantral Program: & WXL-2014-2 & Bandwith: & n.a. \\
Quantif: Method: & WXL & Diution Factor: & 1.0000 \\
Recording Time: & $2014 / 1218$ 17:38 & Sample Weight: & 1.0000 \\
Run Time (min): & 31.76 & Sample Amount: & 1.0000 \\
\hline
\end{tabular}

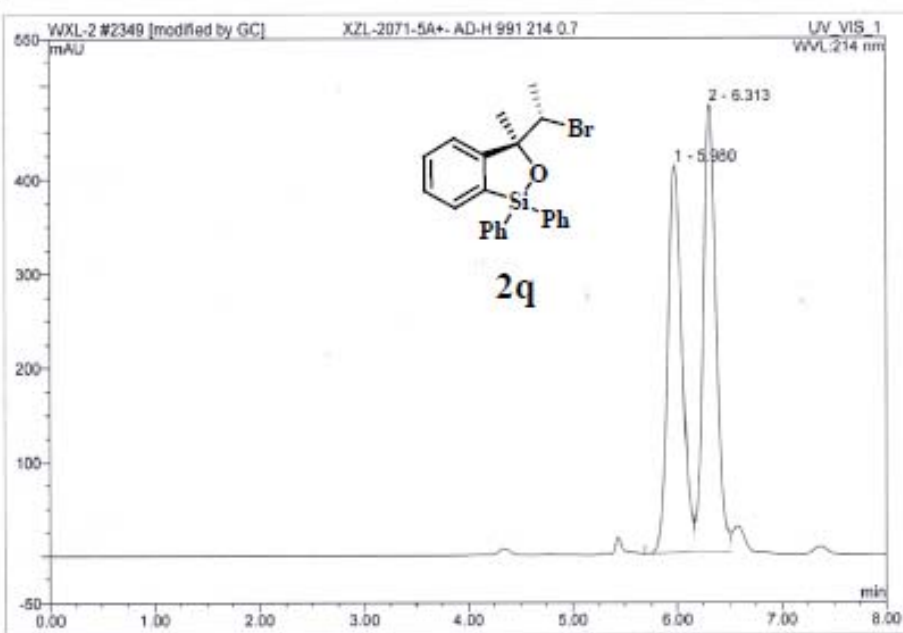

\begin{tabular}{|r|cccrrrr|}
\hline No. & $\begin{array}{c}\text { Ret.Time } \\
\text { min }\end{array}$ & Poak Name & $\begin{array}{c}\text { Height } \\
\text { mAUU }\end{array}$ & $\begin{array}{c}\text { Area } \\
\text { mAU*min }\end{array}$ & $\begin{array}{r}\text { Rel.Area } \\
\%\end{array}$ & Amount & Typo \\
\hline 1 & 5.98 & n.a. & 412.307 & 65.351 & 48.80 & n.a. & BM. \\
2 & 6.31 & n.a. & 475.244 & 68.571 & 51.20 & n.a. & M * $^{*}$ \\
\hline Total: & & & 887.551 & 133.922 & 100.00 & 0.000 & \\
\hline
\end{tabular}




\begin{tabular}{|llll|}
\hline 2350 XZL-2071-5B AD-H 9912140.7 & & \\
& & & \\
\hline Sampie Name: & XZL-2071-5B AD-H 9912140.7 & Injection Volume: & 2.0 \\
Vial Number: & BA2 & Channet: & UV_VIS_1 \\
Sempie Type: & Unknown & Wevelength: & 214 \\
Control Program: & WXL-2014-2 & Bandwidth: & n.a. \\
Quantif. Method: & WXL & Dilution Factor: & 1.0000 \\
Recording Time: & $2014 / 12 / 911: 02$ & Sample Weight: & 1.0000 \\
Run Time (min): & 6.81 & Sample Amount: & 1.0000 \\
\hline
\end{tabular}

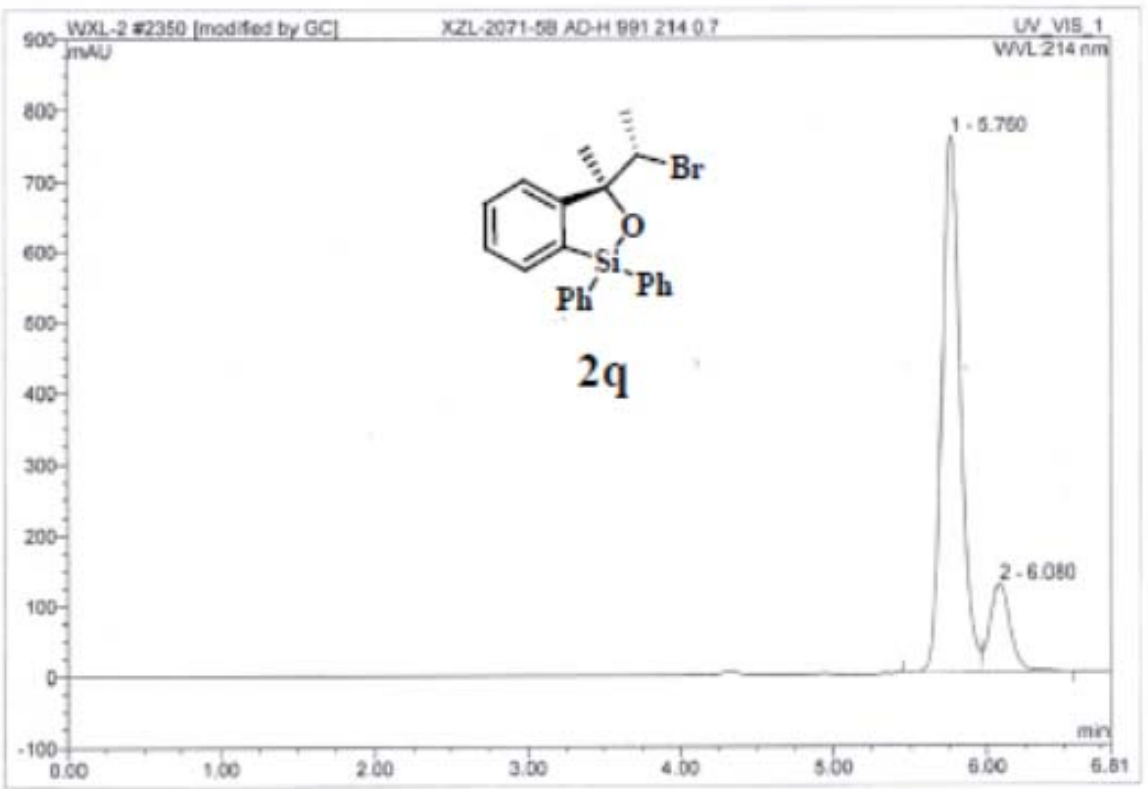

\begin{tabular}{|r|cccrrrrr|}
\hline No. & $\begin{array}{c}\text { Ret.Time } \\
\text { min }\end{array}$ & Peak Name & $\begin{array}{c}\text { Height } \\
\text { mAU }\end{array}$ & $\begin{array}{c}\text { Area } \\
\text { mAU'min }\end{array}$ & $\begin{array}{r}\text { Rol.Aroa } \\
\%\end{array}$ & Amount & Type \\
\hline 1 & 5.76 & n.a. & & 759.823 & 105.803 & 84.83 & n.a. & BM $^{*}$ \\
2 & 6.08 & n.a. & & 126.277 & 18.927 & 15.17 & n.a. & MB* $^{*}$ \\
\hline Total: & & & & 886.100 & 124.730 & 100.00 & 0.000 & \\
\hline
\end{tabular}




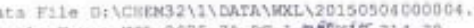

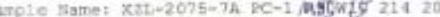

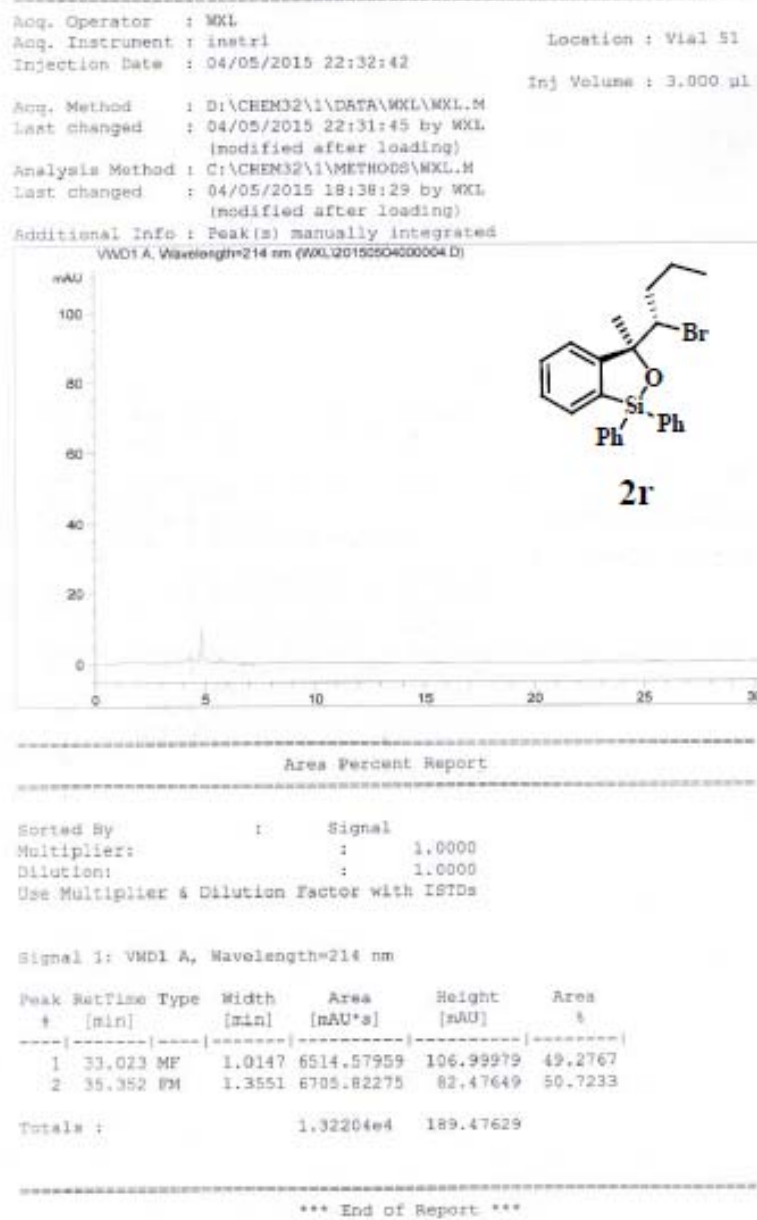

... End of Report $\cdots$ 


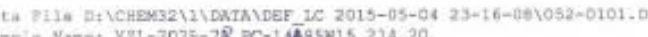

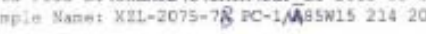

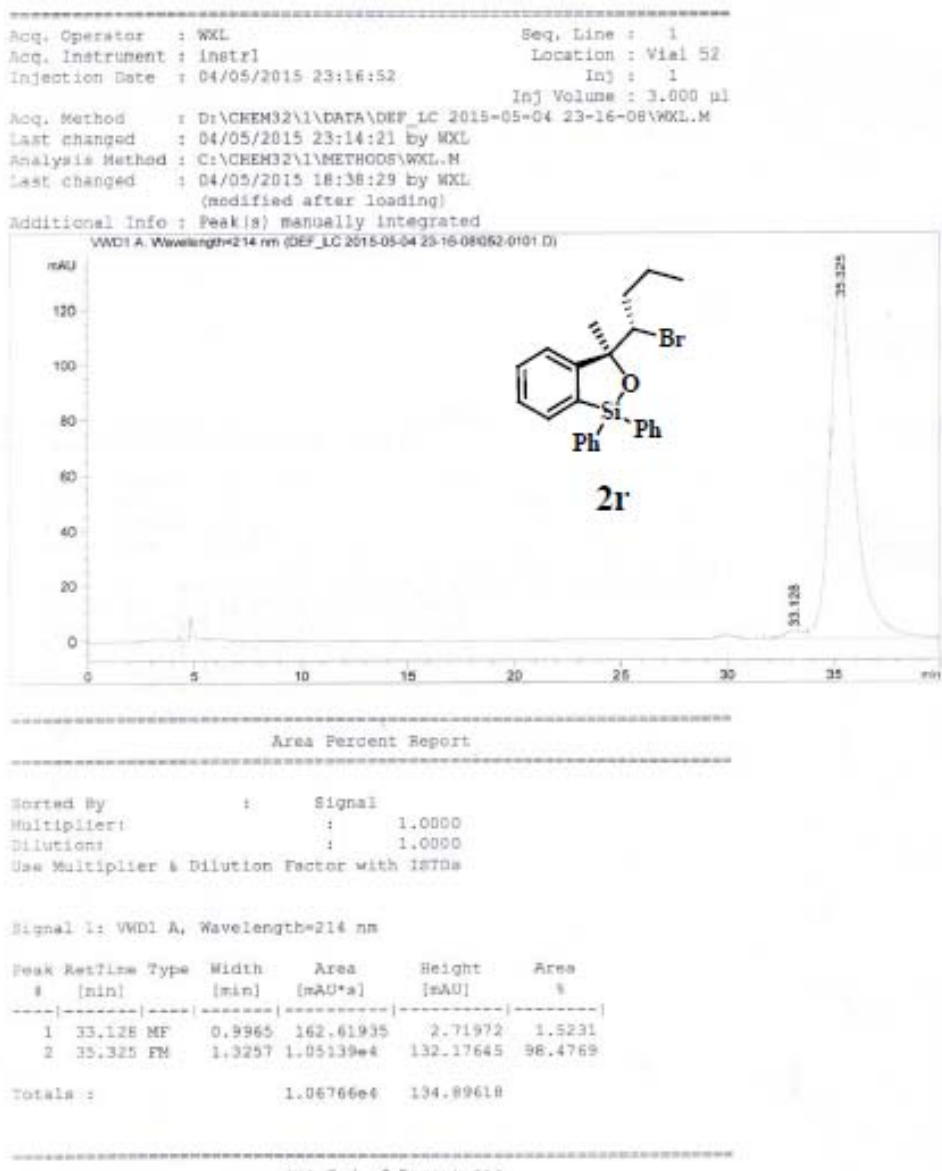

... Ind of Report ... 


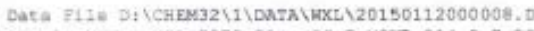
Satple Nana: XZL-2075-2At- PC-3 Menr 2140.720 Mtiw
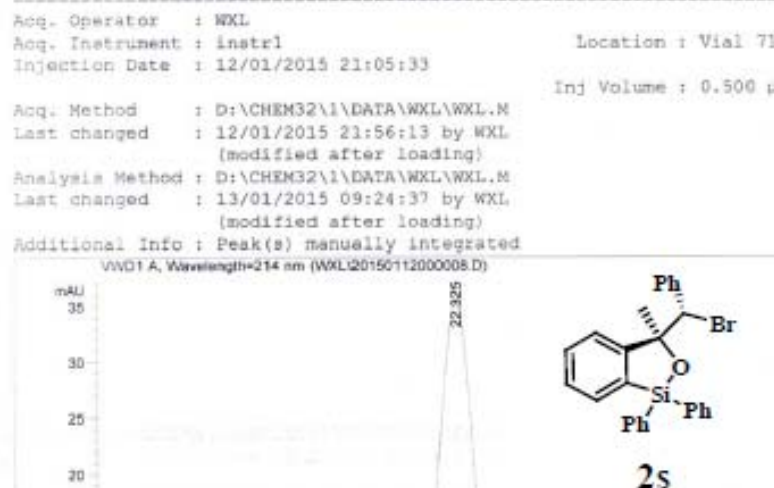

2s

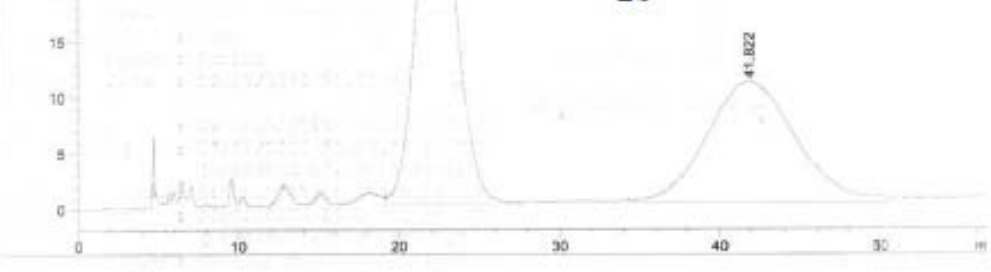

Area Dercent Boport

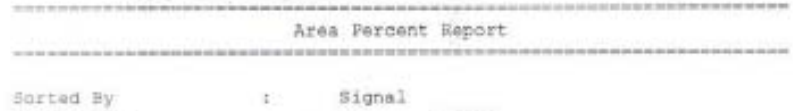

$\begin{array}{lccc}\text { Sorted \#y } & \text { signal } & \\ \text { Sultipliers } & & 3 & 1.0000 \\ \text { Dilutiont } & & ; & 1.0000\end{array}$

Dilutiont

Signal is vwol $\mathrm{k}$, Wavelength=214 nm

\begin{tabular}{|c|c|c|c|c|c|c|}
\hline reak & $\begin{array}{c}\text { RetTime } \\
\text { [nin1 }\end{array}$ & туре & $\begin{array}{l}\text { Mideth } \\
\text { [nin] }\end{array}$ & 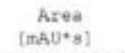 & $\begin{array}{l}\text { Helght } \\
\text { [aAD] }\end{array}$ & $\begin{array}{c}\text { Area } \\
\text { ? }\end{array}$ \\
\hline 1 & 22.325 & $\ln$ & $2,707 \theta$ & 5666.65430 & 34.87867 & 57.7450 \\
\hline 2 & 41.022 & $\mathrm{NM}$ & 6.4349 & 4146.57813 & 10.739988 & 42.2550 \\
\hline & . & & & 9813.23242 & 45.61854 & \\
\hline
\end{tabular}

... End of Roport $\cdots$ 


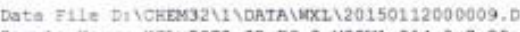
Sarpie Namet X2L-2075-2B PC-3 M99N1 2140.720

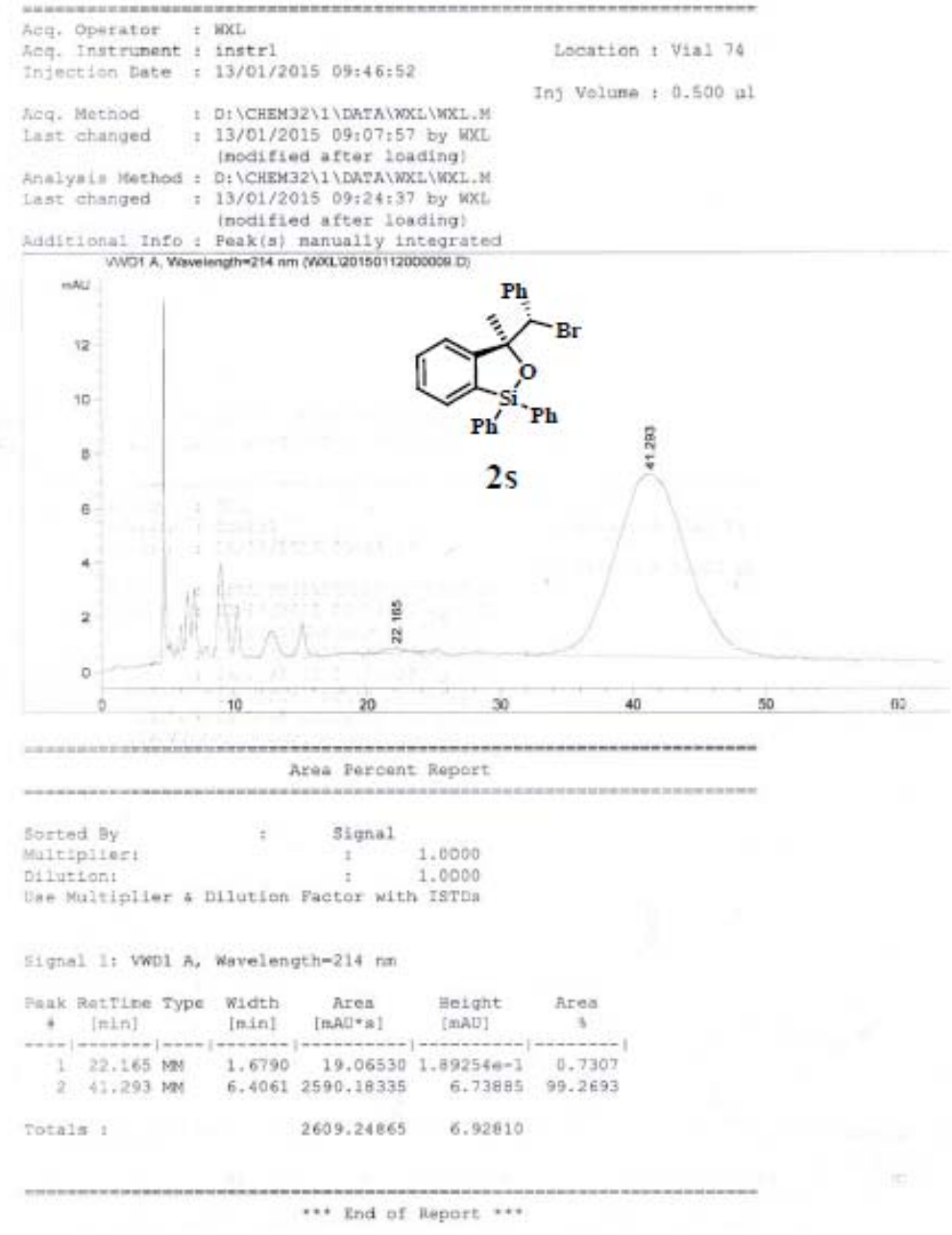




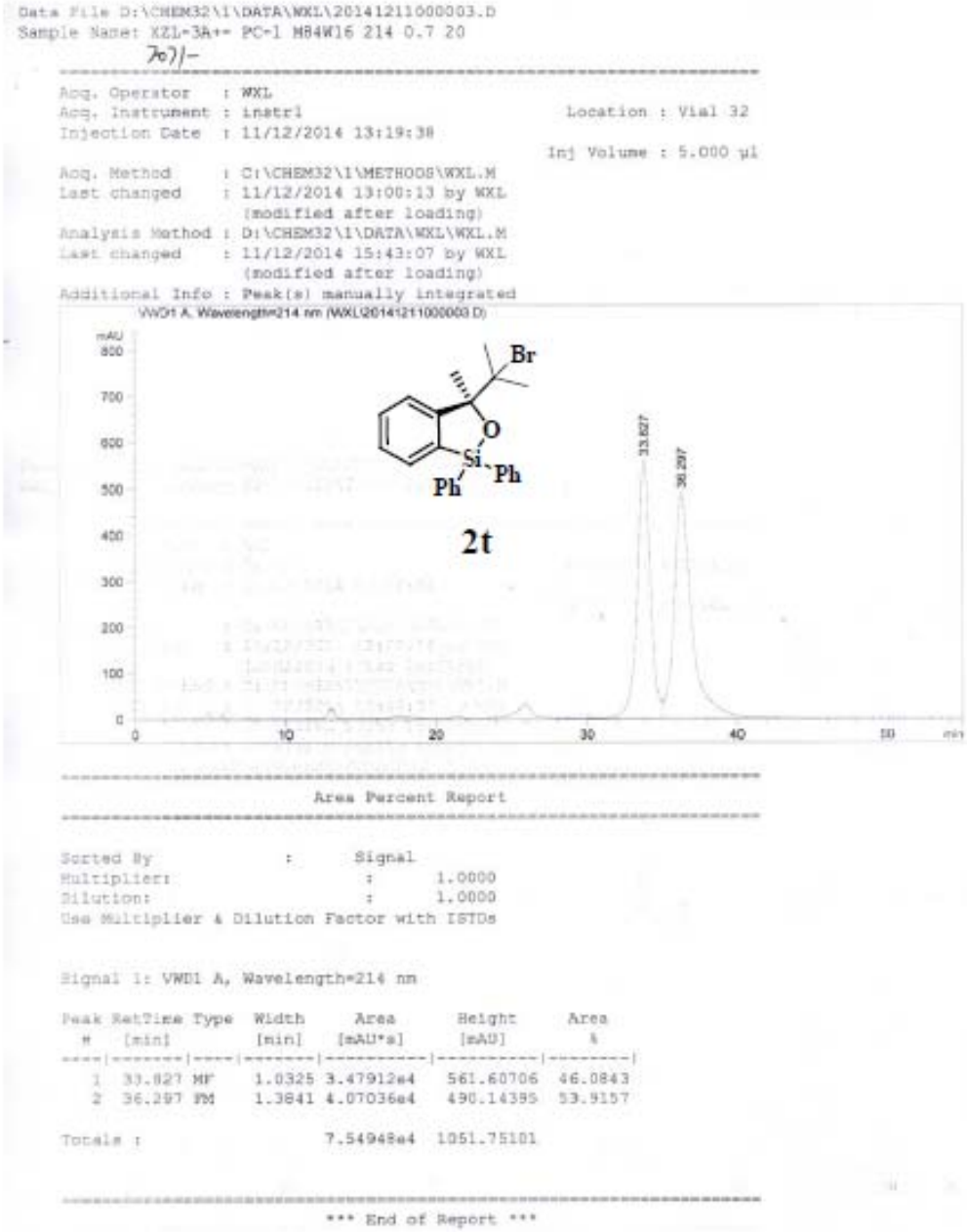




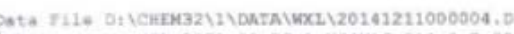

Sarple Sane: X2L-2071-38 PC-1 M541416 $216 \quad 0.729$

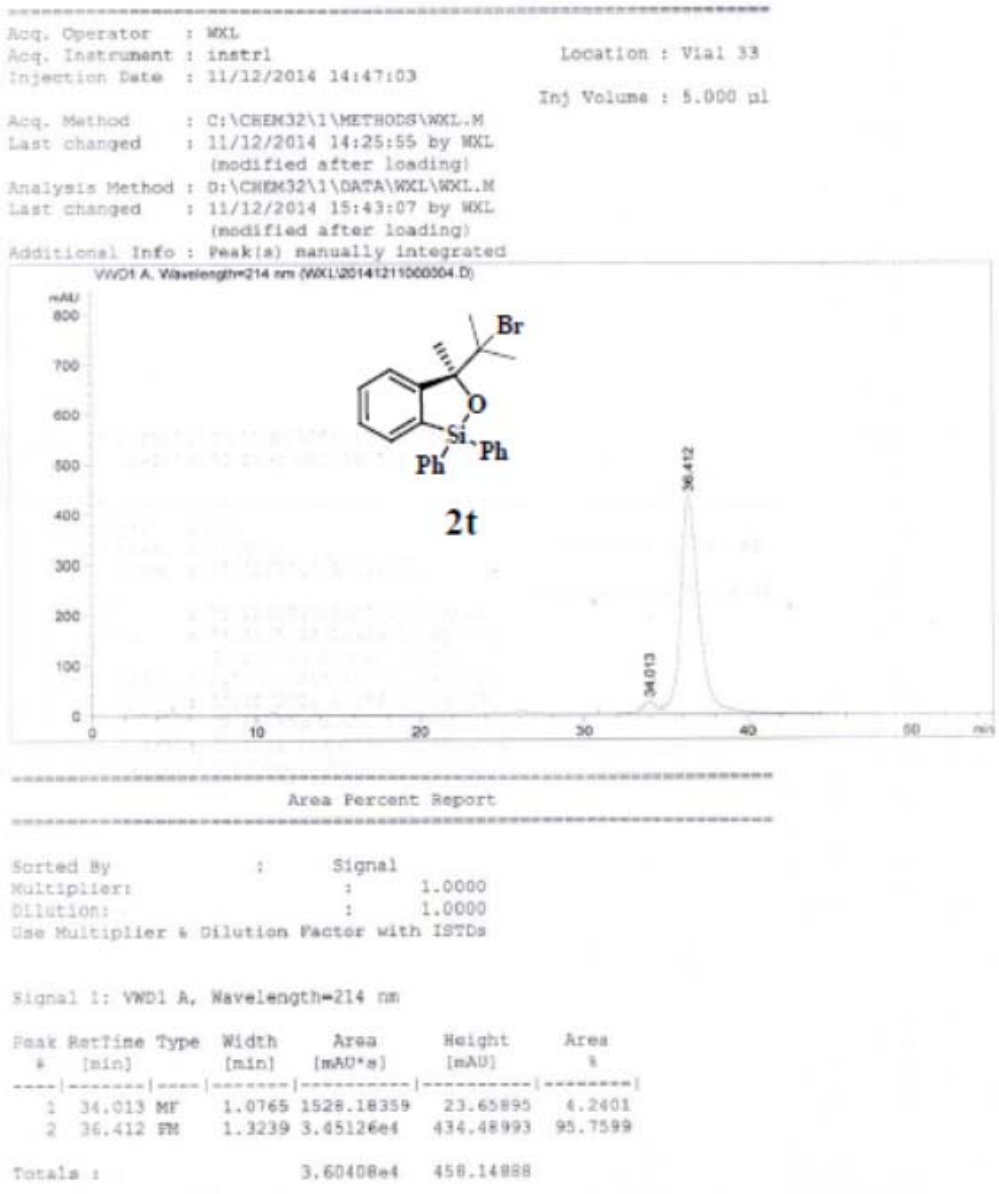

... End of Roport $\cdots$ 


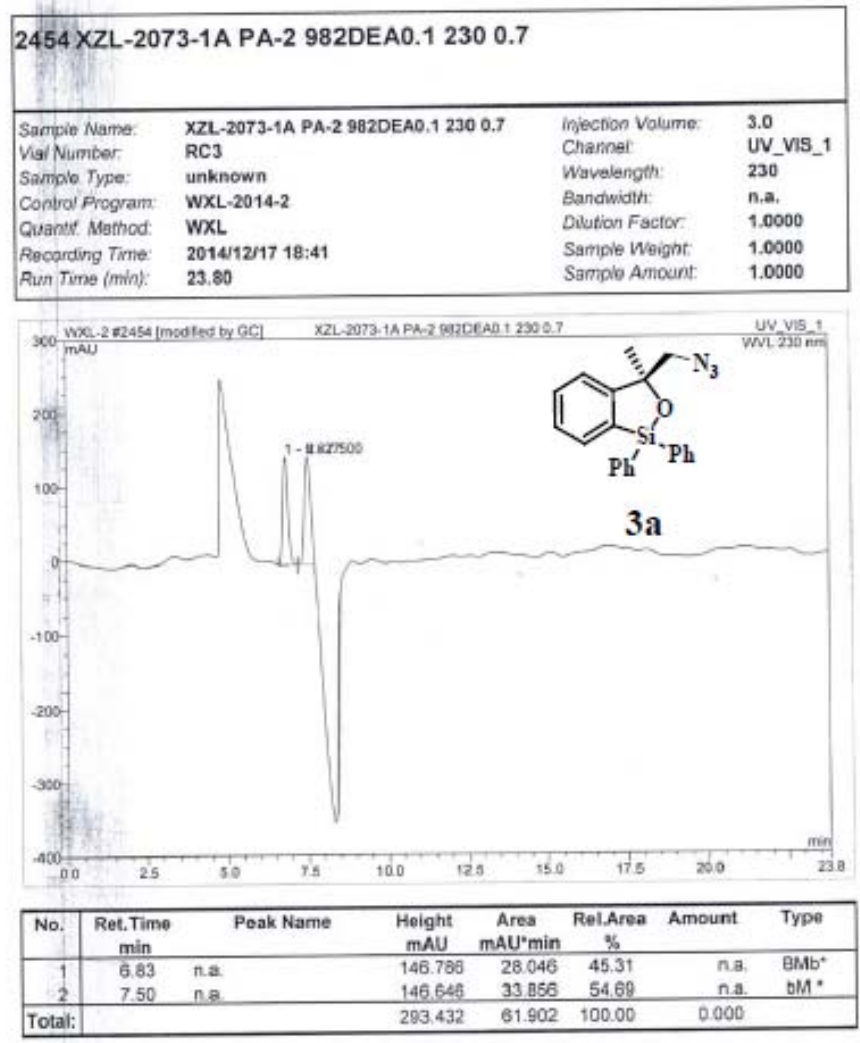




\begin{tabular}{|c|c|c|c|}
\hline \multicolumn{4}{|c|}{2456 XZL-2073-1B PA-2 982DEA0.1 2300.7} \\
\hline & $\begin{array}{l}\text { XZL-2073-1B PA.2 982DEA0.1 } 2300.7 \\
\text { RC5 }\end{array}$ & $\begin{array}{l}\text { Injoction Valume: } \\
\text { Channet: }\end{array}$ & $\begin{array}{l}3.0 \\
\text { UV_VIS_1 }\end{array}$ \\
\hline Sampie Type: & unknown & Wavelength: & 230 \\
\hline Contros Program: & WXL-2014-2 & Bandwiath: & n.a. \\
\hline Quantir Method & wXL. & Dilution Factor, & 1.0000 \\
\hline Recording Time: & 2014/12/17 19:32 & Semplo Weight: & 1.0000 \\
\hline Run Time (min): & 14.91 & Semple Amount: & 1.0000 \\
\hline
\end{tabular}

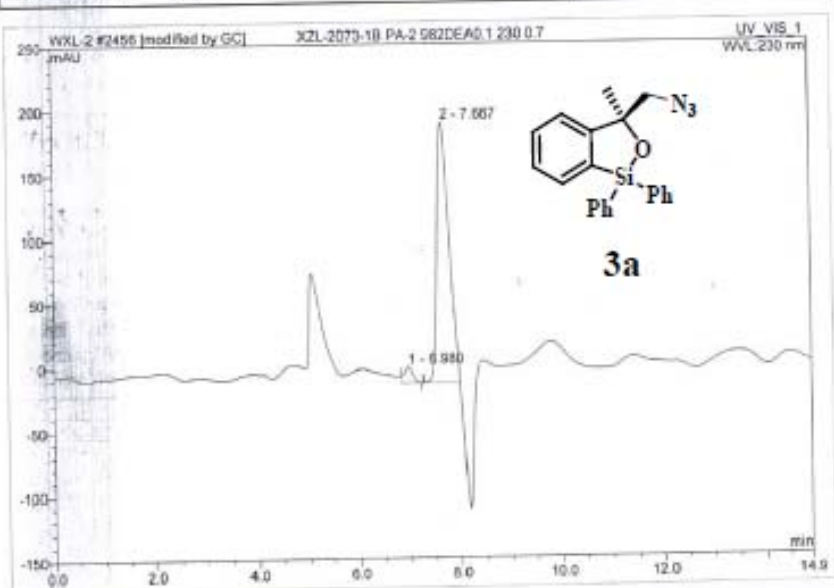

\begin{tabular}{|c|c|c|c|c|c|c|c|}
\hline No. & $\begin{array}{c}\text { Ret Time } \\
\min \end{array}$ & Peak Name & $\begin{array}{c}\text { Height } \\
\text { mAU }\end{array}$ & $\begin{array}{c}\text { Area } \\
\text { mau'min }\end{array}$ & $\begin{array}{c}\text { Rel.Area } \\
\%\end{array}$ & Amount & Type \\
\hline 18 & $\begin{array}{l}6.98 \\
7.87 \\
\end{array}$ & $\begin{array}{l}n a \\
n a\end{array}$ & $\begin{array}{r}11.996 \\
202.221\end{array}$ & $\begin{array}{r}2.512 \\
57.451 \\
\end{array}$ & $\begin{array}{r}4.19 \\
95.81 \\
\end{array}$ & $\begin{array}{l}\text { n.a. } \\
\text { n. }\end{array}$ & $\begin{array}{l}\text { MB: } \\
\text { BM. }\end{array}$ \\
\hline Total: & & & 214.217 & 59.963 & 100.00 & 0.000 & \\
\hline
\end{tabular}


Operator:GC Timobase:U3000 Sequence:WXL-2

Page 1-1
2015/1/8 $9: 24$ 上午

\begin{tabular}{|c|c|c|c|}
\hline \multicolumn{4}{|c|}{2626 XZL-2073-4A+- OD-H 912140.7} \\
\hline $\begin{array}{l}\text { Sampie Name: } \\
\text { Vial Number: } \\
\text { Sarnple Type: } \\
\text { Controi Program: } \\
\text { Quantif. Method: } \\
\text { Recording Time: } \\
\text { Run Time (min): }\end{array}$ & $\begin{array}{l}X Z L-2073-4 A+-O D-H 912140.7 \\
\text { RE1 } \\
\text { unknown } \\
\text { WXL-2014-2 } \\
W X L \\
2015 / 1 / 720: 27 \\
13.44\end{array}$ & $\begin{array}{l}\text { Injection Volume: } \\
\text { Channat: } \\
\text { Wavelengeth: } \\
\text { Bendwiath: } \\
\text { Dilution Factor: } \\
\text { Sample Weight: } \\
\text { Sample Amount: }\end{array}$ & $\begin{array}{l}2.0 \\
\text { UV_Vis_1 } \\
21.4 \\
\text { n.a. } \\
1.0000 \\
1.0000 \\
1.0000\end{array}$ \\
\hline
\end{tabular}

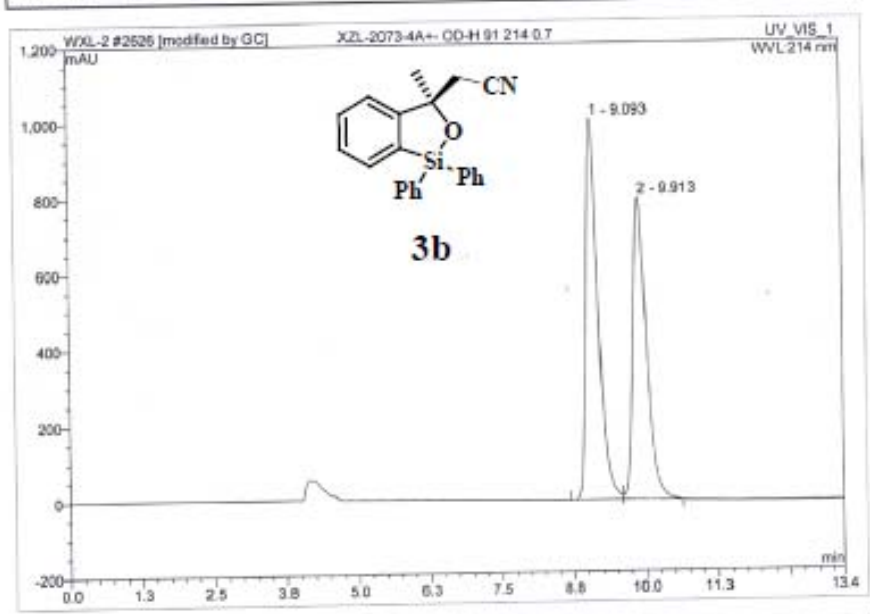

\begin{tabular}{|c|c|c|c|c|c|c|c|}
\hline No. & $\begin{array}{c}\text { Ret. Time } \\
\text { min }\end{array}$ & Peak Name & $\begin{array}{c}\text { Height } \\
\text { mAU }\end{array}$ & $\begin{array}{c}\text { Area } \\
\text { mAU:min }\end{array}$ & $\begin{array}{c}\text { Rol.Area } \\
\%\end{array}$ & Amount & Typo \\
\hline $\begin{array}{l}1 \\
2 \\
\end{array}$ & $\begin{array}{l}9.09 \\
9.91\end{array}$ & $\begin{array}{l}n .8 \\
n .8\end{array}$ & $\begin{array}{r}1009.478 \\
798.641 \\
\end{array}$ & $\begin{array}{r}244.897 \\
212.565 \\
\end{array}$ & $\begin{array}{l}53.53 \\
46.47 \\
\end{array}$ & $\begin{array}{l}n .8 \\
\text { n.a. }\end{array}$ & $\begin{array}{l}\mathrm{BM}^{\circ} \\
\mathrm{MB}\end{array}$ \\
\hline to & & & 1808.119 & 457.461 & 100.00 & 0.000 & \\
\hline
\end{tabular}


Operator:GC Timebase U3000 Sequence:WXL-2

Poge 1-1

\begin{tabular}{|c|c|c|c|}
\hline \multicolumn{4}{|c|}{2627 XZL-2073-4B OD-H 912140.7} \\
\hline $\begin{array}{l}\text { Samplo Narne: } \\
\text { Vial Number: } \\
\text { Sampie Type: } \\
\text { Cantrol Program: } \\
\text { Quanth. Mothod: } \\
\text { Recarding Time: } \\
\text { Run Tine (min): }\end{array}$ & $\begin{array}{l}X Z L-2073-4 B \text { OD-H } 912140.7 \\
\text { RD1 } \\
\text { unknown } \\
\text { WXL-2014-2 } \\
\text { WXL } \\
201511 / 720: 42 \\
20.22\end{array}$ & $\begin{array}{l}\text { Injoction Volume: } \\
\text { Channet: } \\
\text { Wavefongth: } \\
\text { Bandwith: } \\
\text { Ditution Factor: } \\
\text { Sample Weight } \\
\text { Sample Amount: }\end{array}$ & $\begin{array}{l}1.0 \\
\text { UV_VIS_1 } \\
214 \\
\text { n.a. } \\
1.0000 \\
1.0000 \\
1.0000\end{array}$ \\
\hline
\end{tabular}

$x 2-2072-1800+492140.7$

WV v15:

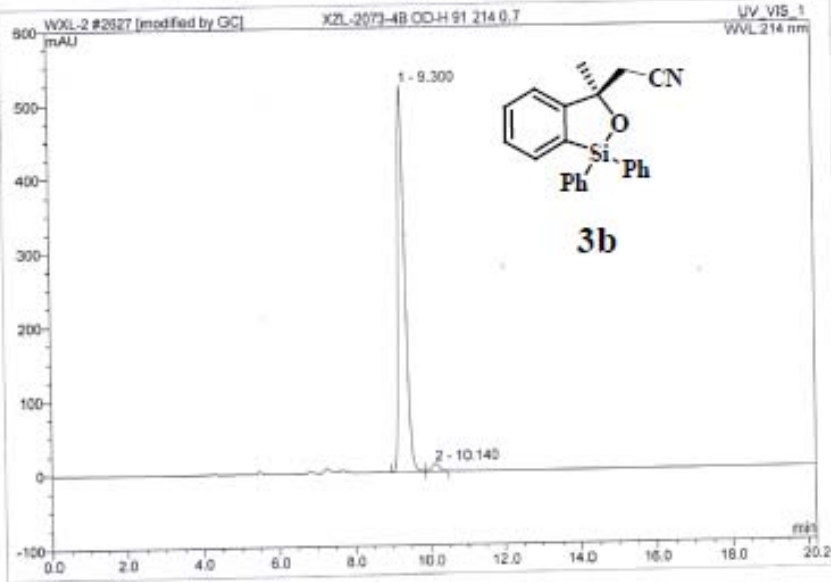

\begin{tabular}{|c|c|c|c|c|c|c|c|}
\hline No. & $\begin{array}{c}\text { Ret Time } \\
\min \end{array}$ & Peak Name & $\begin{array}{c}\text { Height } \\
\text { mAU }\end{array}$ & $\begin{array}{c}\text { Area } \\
\text { maU*min }\end{array}$ & $\begin{array}{c}\text { Rel.Area } \\
\quad \%\end{array}$ & Amount & Type \\
\hline otal: & & & 533.764 & 116.679 & 100.00 & 0.000 & \\
\hline
\end{tabular}




\begin{tabular}{|c|c|c|c|}
\hline \multicolumn{4}{|c|}{3929 XZL-2073-2A OD-H 912140.7} \\
\hline Sample Name: & $X Z L-2073-2 A$ OD-H 912140.7 & miection valume: & 5.0 \\
\hline Vial Number: & RBs & Channel: & UV_VIS_2 \\
\hline Sample Type: & unknown & Waveiength: & 214.0 \\
\hline Control Program & test-dad2 & Banolwidth: & 4 \\
\hline Quantit. Method: & wXL & Dilution Factor: & 1.0000 \\
\hline Recarding Tine: & $2015-1-5 \quad 15: 50$ & Sample Weight: & 1.0000 \\
\hline Run The (min): & 15.00 & Simplo Amount: & 1.0000 \\
\hline
\end{tabular}

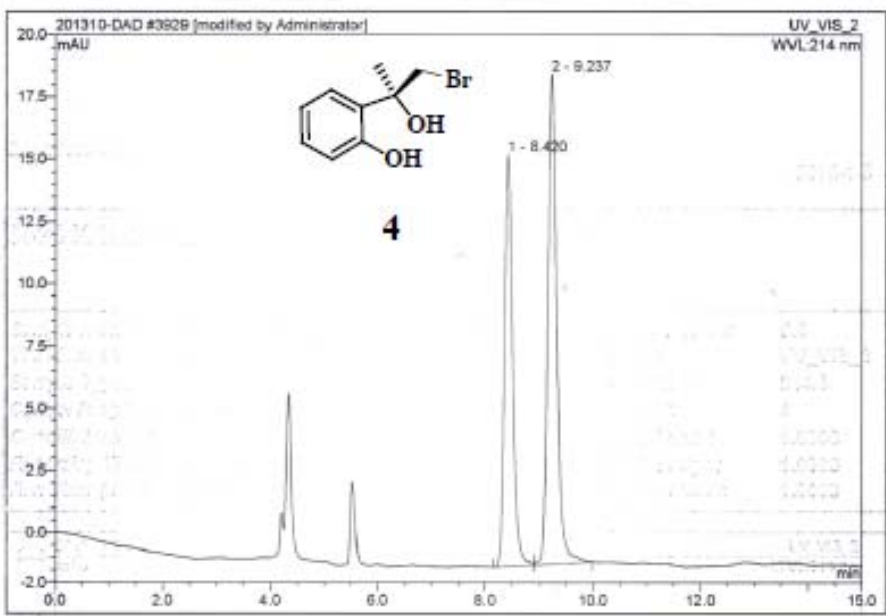

\begin{tabular}{|r|cccrrrr|}
\hline No. & $\begin{array}{c}\text { Ret. Time } \\
\text { min }\end{array}$ & Peak Name & $\begin{array}{c}\text { Height } \\
\text { mAU }\end{array}$ & $\begin{array}{c}\text { Area } \\
\text { mAU*min }\end{array}$ & $\begin{array}{r}\text { Rel.Area } \\
\%\end{array}$ & Amount & Type \\
\hline 1 & 8.42 & n.a. & 16.508 & 2.964 & 43.02 & n.a. & M * $^{*}$ \\
2 & 9.24 & n.a. & 19.626 & 3.926 & 56.98 & n.a. & MB* \\
\hline Total: & & & 36.135 & 8.890 & 100.00 & 0.000 & \\
\hline
\end{tabular}




\begin{tabular}{|c|c|c|c|}
\hline \multicolumn{4}{|c|}{3930 XZL-2073-2B OD-H 912140.7} \\
\hline Semple Name: & XZL-2073-2B OD-H 912140.7 & injection Volume: & 5.0 \\
\hline Vial Number: & RAS & Channe: & UV_VIS_2 \\
\hline Sample Type: & unknown & Wavelengem: & 214.0 \\
\hline Control Program: & test-dad2 & Bandwitth: & 4 \\
\hline Quantif Mothod & wXL. & aivition Factor: & 1.0000 \\
\hline Rocarding Time: & $2015-1-5$ 16:05 & Samale Waight: & 1.0000 \\
\hline Rum Time (min): & 15.00 & Samale Amount: & 1.0000 \\
\hline
\end{tabular}

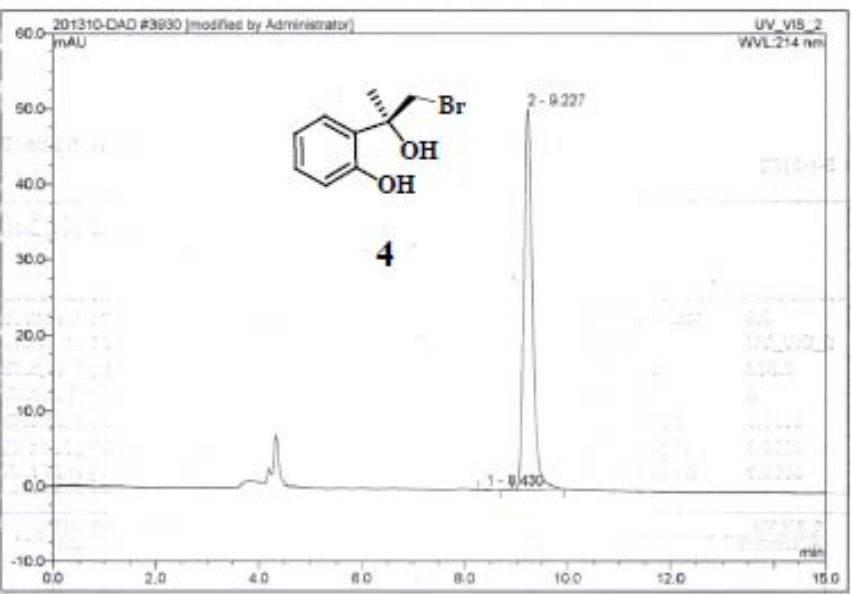

\begin{tabular}{|c|c|c|c|c|c|c|c|}
\hline No. & $\begin{array}{c}\text { Ret. Time } \\
\text { min }\end{array}$ & Peak Name & $\begin{array}{l}\text { Height } \\
\text { MAU }\end{array}$ & $\begin{array}{c}\text { Area } \\
\text { mAU"min }\end{array}$ & $\begin{array}{c}\text { Rei_Area } \\
\%\end{array}$ & Amount & Type \\
\hline 1 & 8.43 & n.a. & 0.114 & 0.018 & 0.18 & ก. & BMB' \\
\hline 2 & 9.23 & na. & 50.438 & 9.980 & 99.82 & n.a & BMB \\
\hline Total: & & & 50.551 & 9.998 & 100.00 & 0.000 & \\
\hline
\end{tabular}




\begin{tabular}{|c|c|c|c|}
\hline \multicolumn{4}{|c|}{3949 XZL-2074-2A+- ID3 912140.7} \\
\hline $\begin{array}{l}\text { Sample Narne: } \\
\text { Vial Number: }\end{array}$ & $\begin{array}{l}\text { XZL-2074-2A+- 1D391 } 2140.7 \\
\text { RC4 }\end{array}$ & $\begin{array}{l}\text { Injection Volume: } \\
\text { Channet }\end{array}$ & $\begin{array}{l}2.0 \\
\text { UV_VIS_2 }\end{array}$ \\
\hline Sample Type: & unknown & Waveilength: & 214.0 \\
\hline Contrai Program: & test-dad & Bandwiath: & 4 \\
\hline Quantir Methat & $w \times L$ & Diurion Factor: & 1.0000 \\
\hline & $2015-1-8$ 14:19 & Sample Weight: & 1.0000 \\
\hline Rin TMe (min): & 15.26 & Simple Amount: & 1.0000 \\
\hline
\end{tabular}

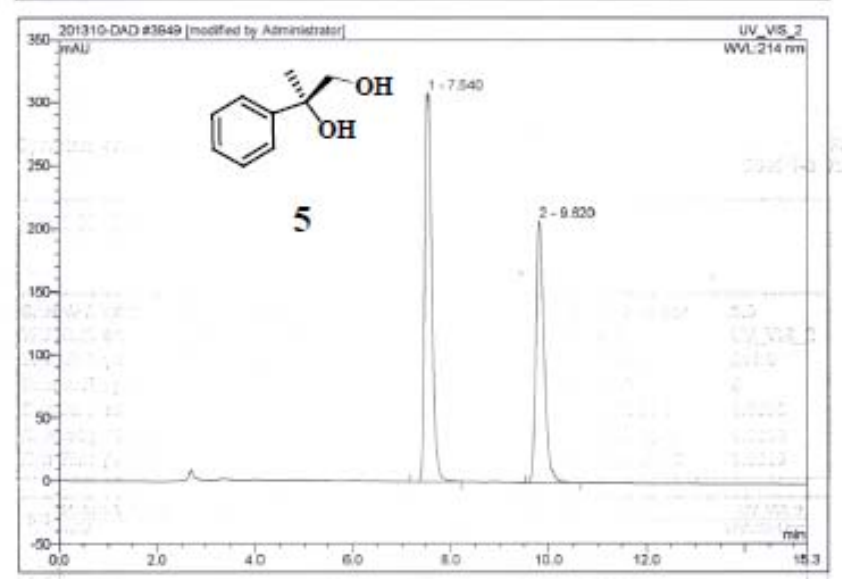

\begin{tabular}{|c|c|c|c|c|c|c|c|}
\hline No. & $\begin{array}{c}\text { Ret. Time } \\
\text { min }\end{array}$ & Peak Name & $\begin{array}{c}\text { Height } \\
\text { mAU }\end{array}$ & $\begin{array}{c}\text { Area } \\
\text { mAU:min }\end{array}$ & $\begin{array}{c}\text { Rei_area } \\
x\end{array}$ & Amount & Type \\
\hline 1 & 7.54 & n.8. & 308.302 & 48.351 & 53.15 & n.. & BM. \\
\hline 2 & 9.82 & n.a. & 207.579 & 40.864 & 46.85 & na. & BME \\
\hline Total: & & & 515.881 & 87.215 & 100.00 & 0.000 & \\
\hline
\end{tabular}




\begin{tabular}{|c|c|c|c|}
\hline \multicolumn{4}{|c|}{3950 XZL-2074-2B ID3 912140.7} \\
\hline Sample Name: & XZL-2074-28 ID3 912140.7 & injection Voutume: & 2.0 \\
\hline Val Number: & RBA & Channet: & UV_vis_z \\
\hline Sample Type: & unknown & Wavelength: & 214.0 \\
\hline Control Program: & test-dad & Bsnowiolth: & 4 \\
\hline Quantif Method: & $w \times L$ & Dilution Factor. & 1.0000 \\
\hline Recording Time: & 2015-1-8 14:38 & Sample Waignt: & 1.0000 \\
\hline Run Time (min): & 14.46 & Sample Amount: & 1.0000 \\
\hline
\end{tabular}

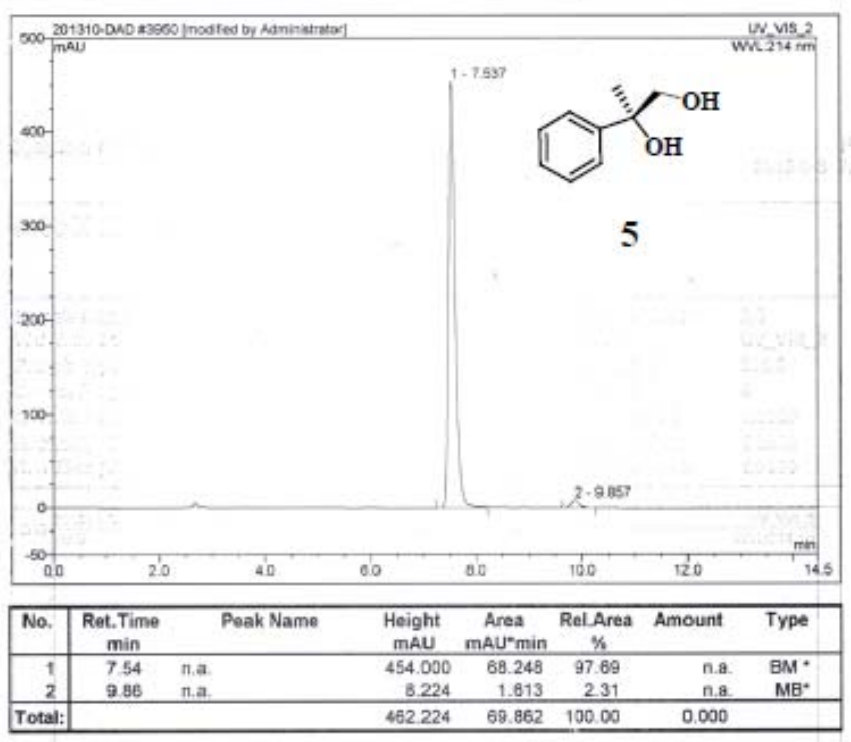


Operator:GC Tmebase:U3000 Sequance WXX-2

Page 1-1
$2015 / 3 / 10$ 10:16 上午

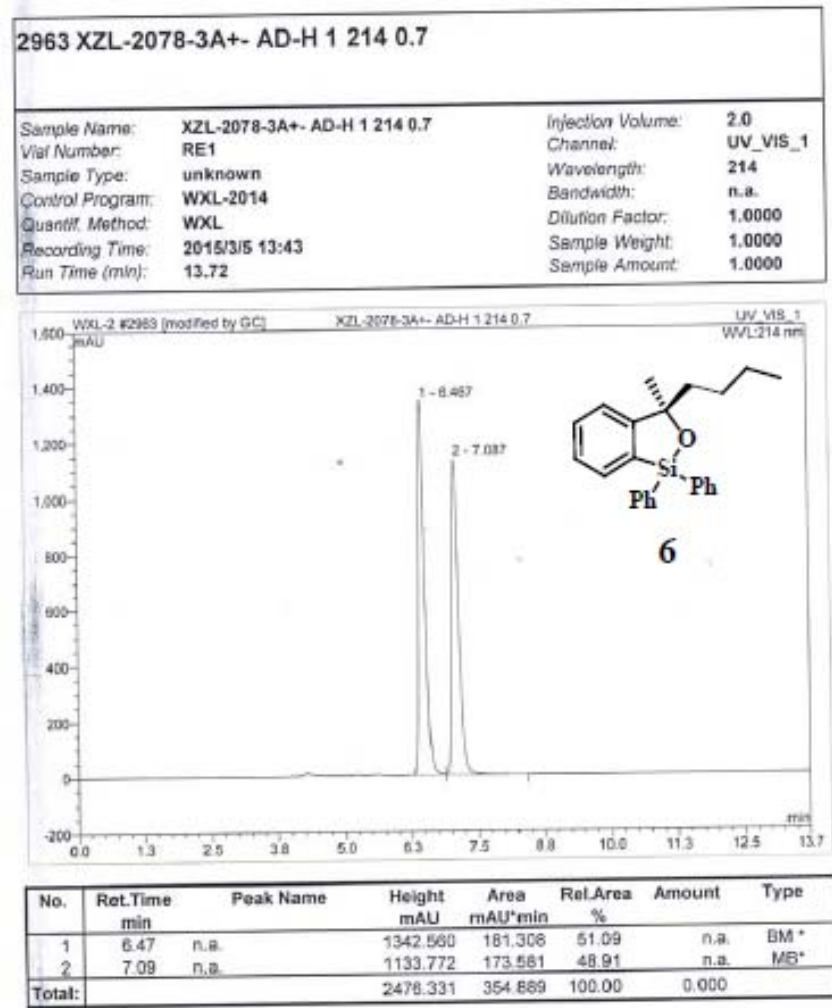




\begin{tabular}{|c|c|c|c|}
\hline \multicolumn{4}{|c|}{2964 XZL-2078-3B AD-H 12140.7} \\
\hline $\begin{array}{l}\text { Sampie Name: } \\
\text { Vial Nuwnoer: } \\
\text { Sample Type: } \\
\text { Control Program: } \\
\text { Quantit. Method: } \\
\text { Recording Time: } \\
\text { Run Tine (min): }\end{array}$ & $\begin{array}{l}X Z L-2078-3 B \text { AD-H } 12140.7 \\
\text { RE2 } \\
\text { unknown } \\
\text { WXL-2014 } \\
\text { WXL } \\
2015 / 3 / 514: 00 \\
9.31\end{array}$ & $\begin{array}{l}\text { Injection Volume: } \\
\text { Channel: } \\
\text { Wavelength: } \\
\text { Bandwidth: } \\
\text { Oihtion Factor: } \\
\text { Sempie Weight: } \\
\text { Sample Amount }\end{array}$ & $\begin{array}{l}2.0 \\
\text { UV_VIS_1 } \\
214 \\
\text { n.a. } \\
1.0000 \\
1.0000 \\
1.0000\end{array}$ \\
\hline
\end{tabular}

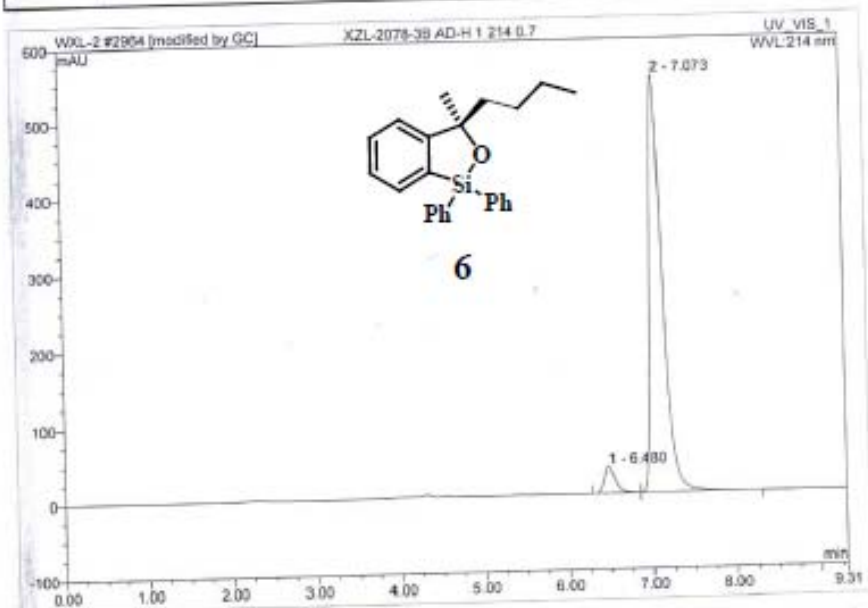

\begin{tabular}{|c|c|c|c|c|c|c|c|}
\hline No. & $\begin{array}{c}\text { Ret.Time } \\
\text { min }\end{array}$ & Peak Name & $\begin{array}{l}\text { Height } \\
\text { mau }\end{array}$ & $\begin{array}{c}\text { Area } \\
\text { mau'min }\end{array}$ & $\begin{array}{c}\text { Rol.Area } \\
\%\end{array}$ & Amount & Type \\
\hline Total: & & & 585.404 & 99.039 & 100.00 & 0.000 & \\
\hline
\end{tabular}


Datz File D: \CHEMa2 \IDATA \Y2R $\backslash 20150416000004$.D
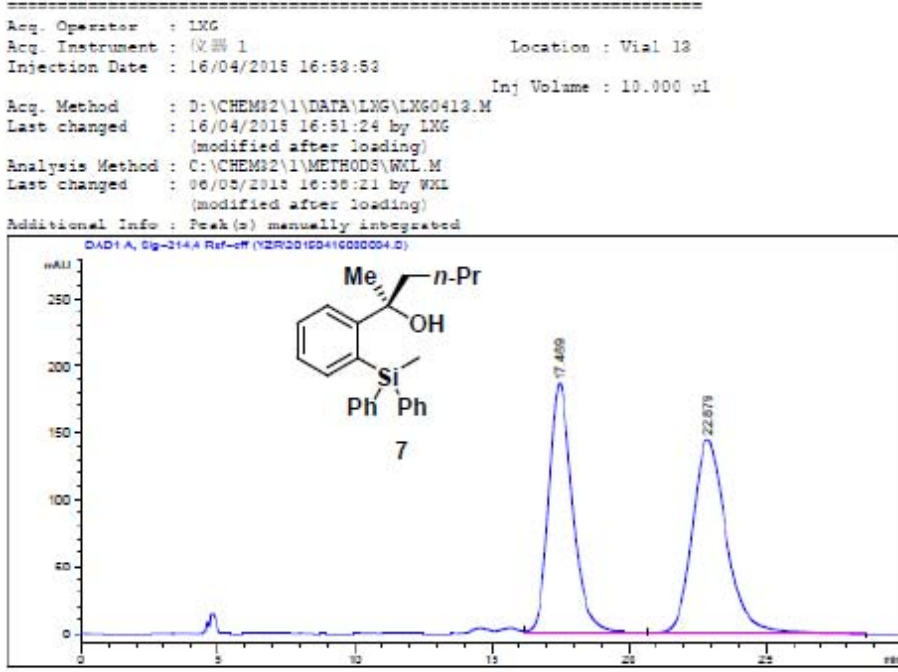

hese Deresent Daport

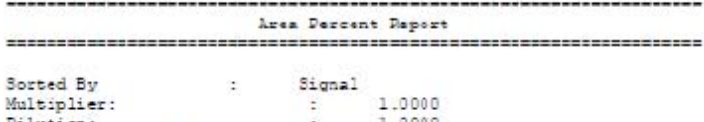

Multiplier

To bultiolier a Dilution Factor with

S1gnai 1: DaDi A, stg-214,4 Net-ort

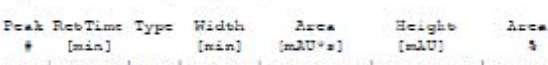

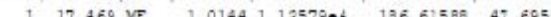

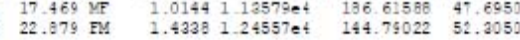

Tocais : $\quad 2.36137 \mathrm{et} \quad 331.40610$ 


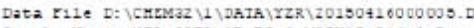

Sample 17:
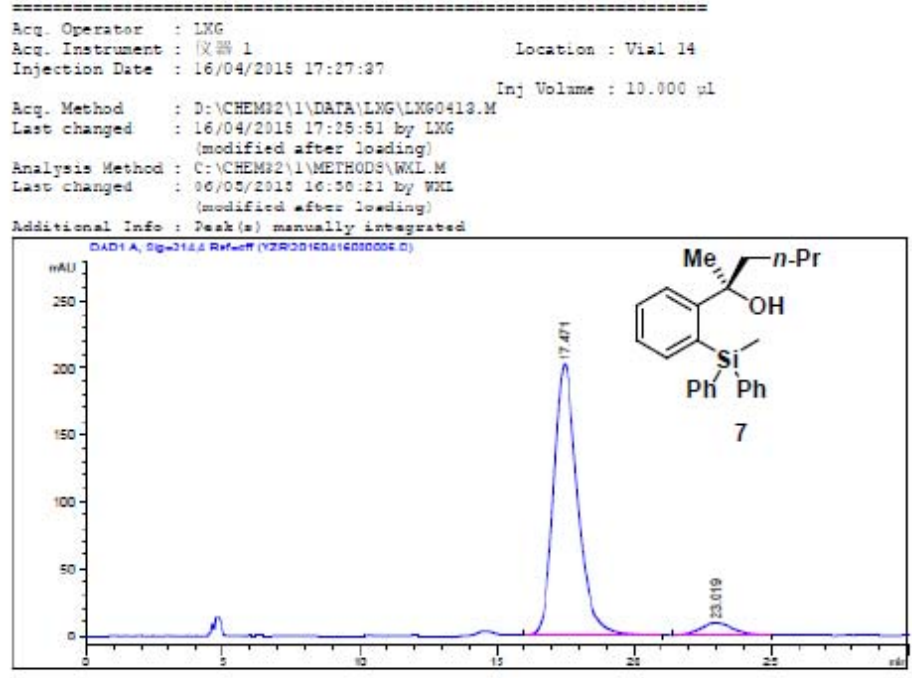

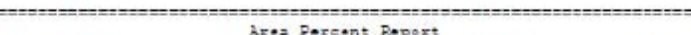

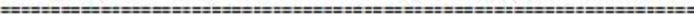

Sorted By

Signa:

Multiplier:

7o. Hultiplier a Dilution Factor with I3TD

Bigne1 1: DADI A, Big-214,4 Ref-oft

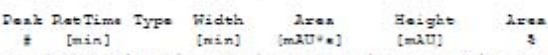

$\begin{array}{llllll}1 & 17.471 \text { EB } & 0.8966 & 1.22270 \mathrm{e} & 202.17014 & 94.5279\end{array}$

Totals : $\quad 1.29343 e 4 \quad 210.64236$ 


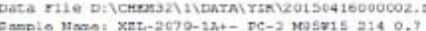

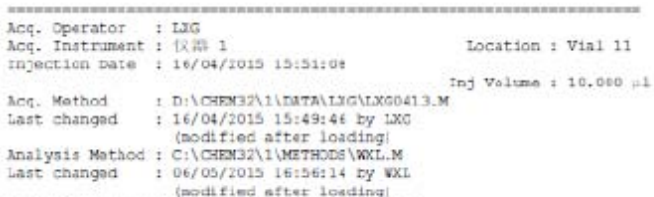

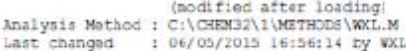
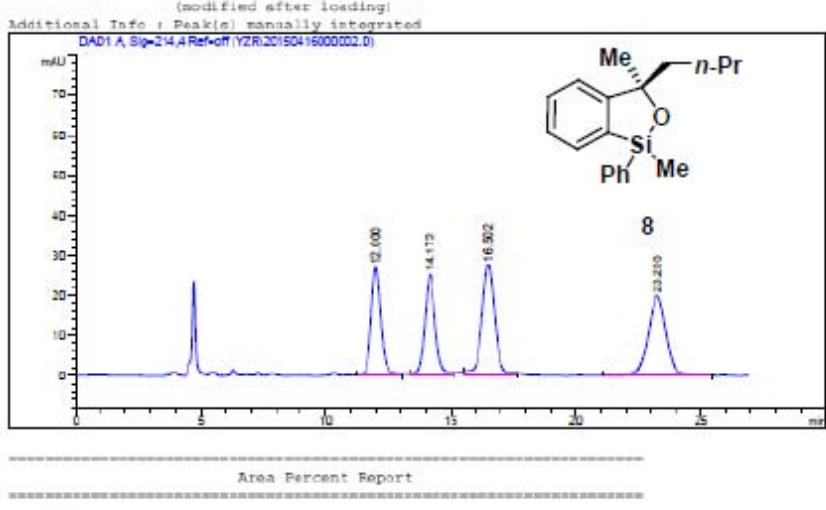

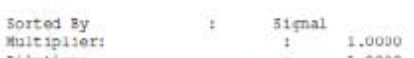

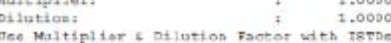

31gnal it: DNo1 $\pi$, sigme214, 4 Rotoott

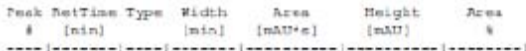

$\begin{array}{lllllll}12.000 \mathrm{Mg} & 0.4478 & 725.49792 & 27.00406 & 20.8421\end{array}$

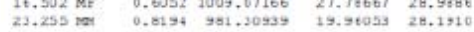

rotala : $3487.92877 \quad 100.12579$

*. rua or report $\cdots$ 


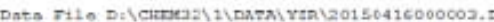

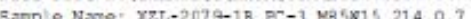
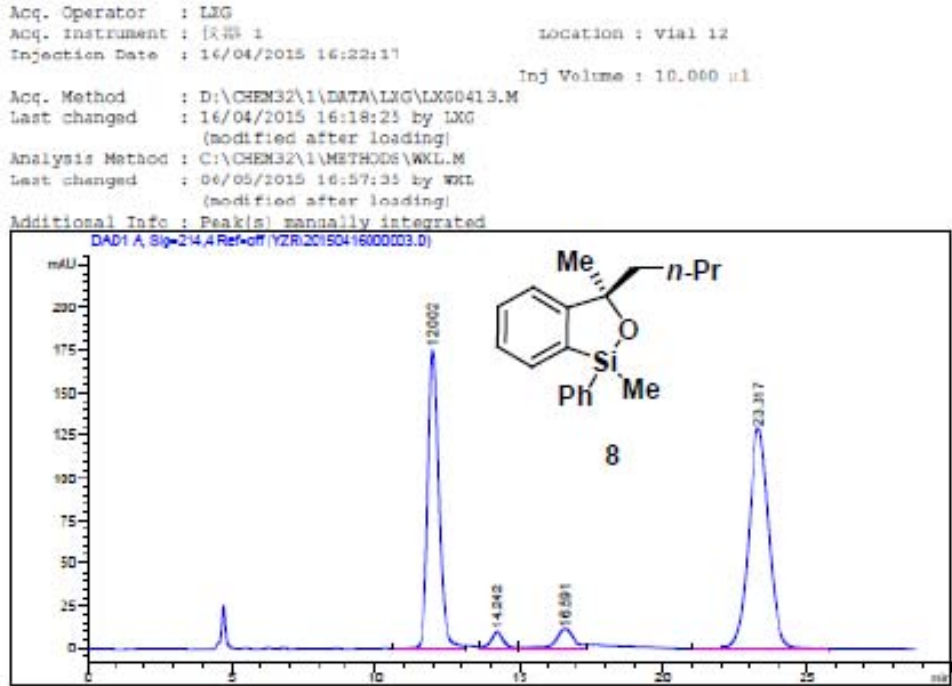

Aroa Percent Feport

sorted $\mathrm{zy}$

Multiplier:

sişnai

1. 1.0000

Dae Multiplier 6 Dilution Factor with rspos

S1gnal 1: DNO1 A, 81gme14, 4 Rataott

\begin{tabular}{|c|c|c|c|c|c|}
\hline Ponk & $\begin{array}{l}\text { Rotrino Typo } \\
\text { [nin] }\end{array}$ & $\begin{array}{l}\text { Midth } \\
\text { |nin| }\end{array}$ & $\begin{array}{l}\text { Ares } \\
{[\operatorname{man})^{*}=1}\end{array}$ & $\begin{array}{l}\text { Ro: ght } \\
\text { [nall) }\end{array}$ & Aros \\
\hline & $12.002 \mathrm{MF}$ & 0.4275 & $4484+25723$ & 174.82791 & $39.1: 24$ \\
\hline 2 & $14.242 \mathrm{NF}$ & 0.5322 & 301.11169 & 9.42981 & 2.6311 \\
\hline & $10.591 \mathrm{rar}$ & 0.7303 & 481,01590 & 10.88851 & 4.2031 \\
\hline+ & 23.317001 & 0.7202 & $6172+04240$ & 120.71962 & 53.9124 \\
\hline
\end{tabular}

Totals :

$3.1444304 \quad 323.84593$

A. nind of Report *.. 


\section{ORTEP drawings of 20}
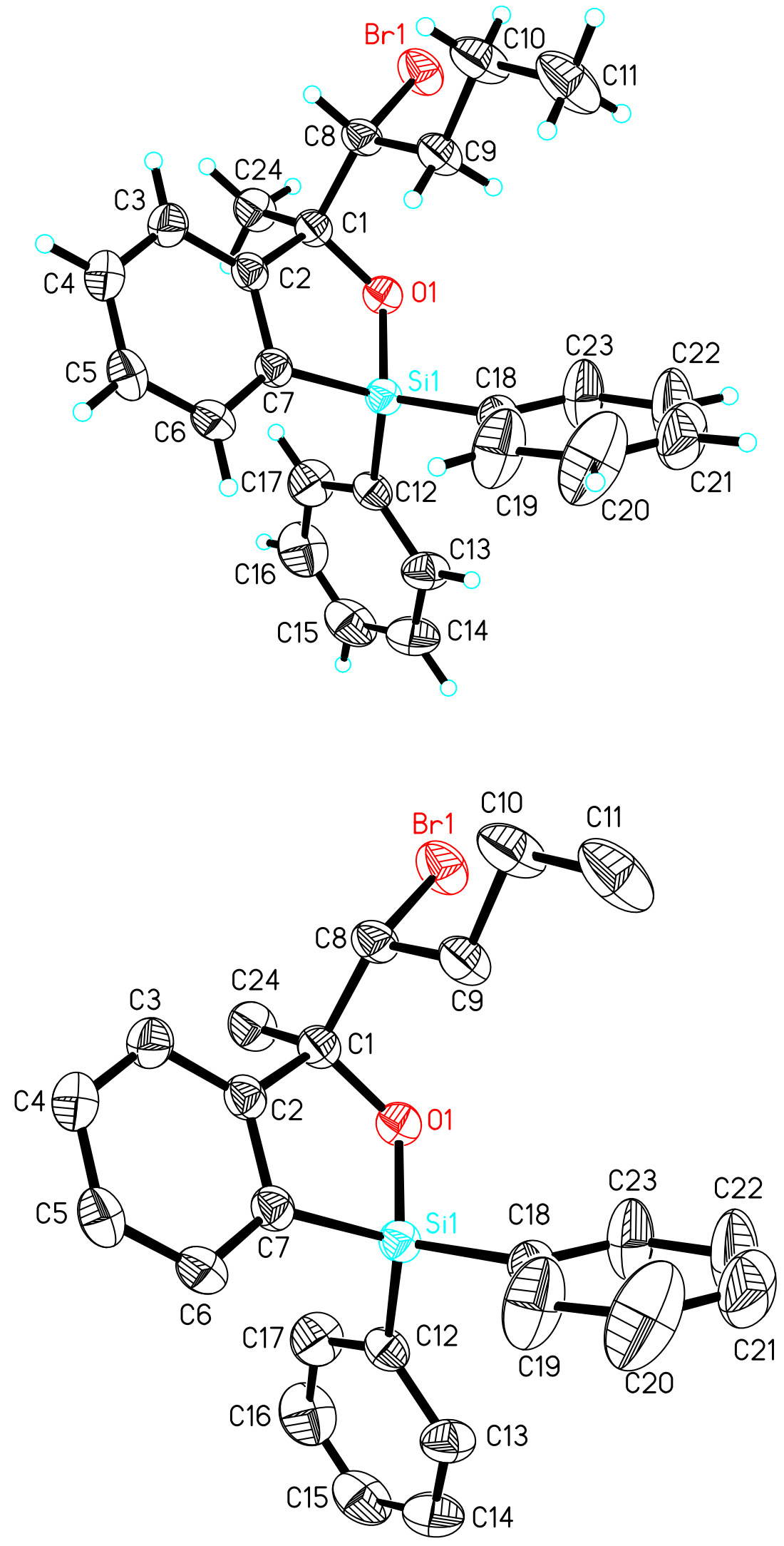

Figrue S3. ORTEP drawing of $2 \mathbf{0}$ 


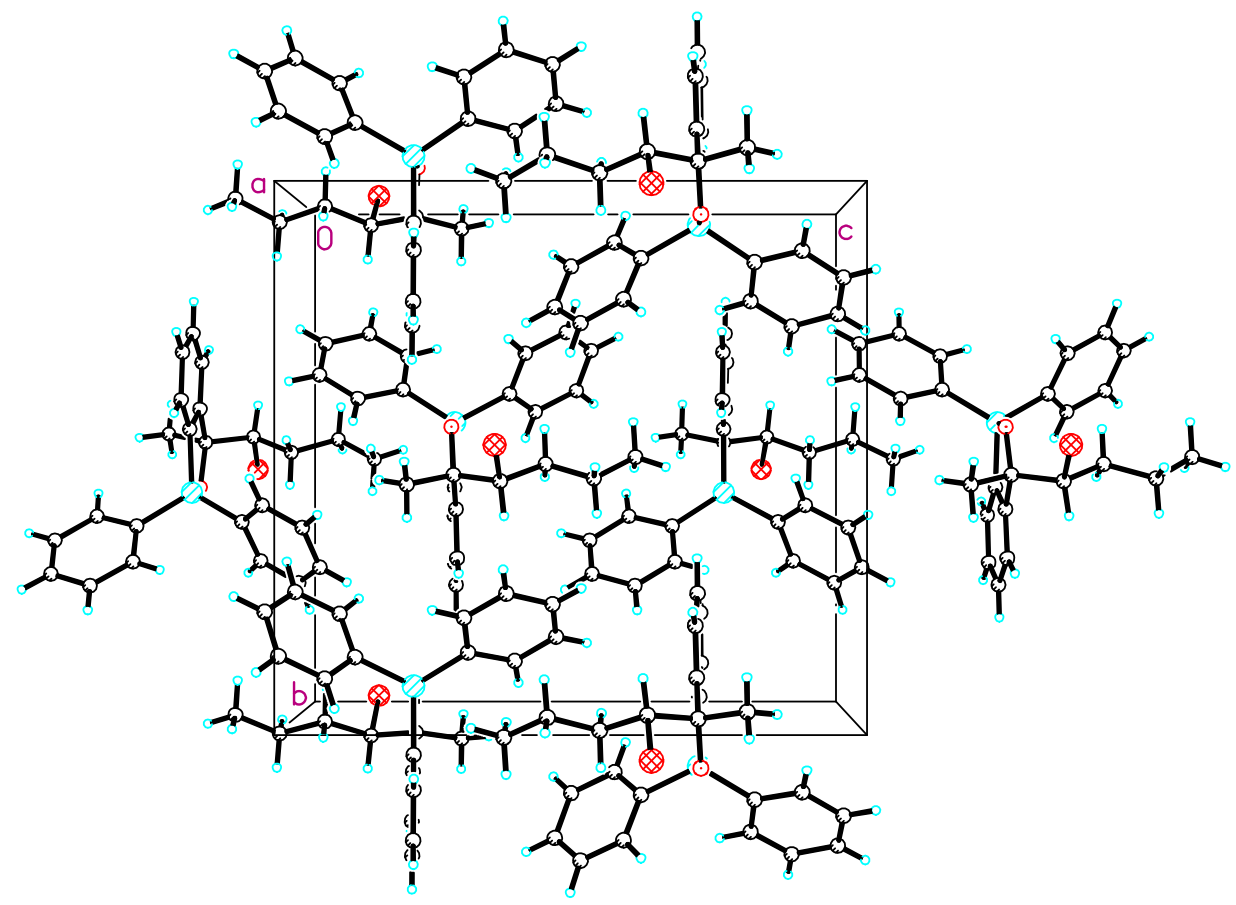

Figrue S4. X-ray crystallography of 20 